\title{
The MicroBooNE Search For Anomalous Electron Neutrino Appearance Using Image Based Data Reconstruction
}

\author{
Victor Genty
}

Submitted in partial fulfillment of the

requirements for the degree

of Doctor of Philosophy

in the Graduate School of Arts and Sciences

\section{COLUMBIA UNIVERSITY}


(C) 2019

Victor Genty

All Rights Reserved 


\section{ABSTRACT}

\section{The MicroBooNE Search For Anomalous Electron Neutrino Appearance Using Image Based Data Reconstruction}

\section{Victor Genty}

This thesis presents the MicroBooNE search for the MiniBooNE low energy excess using a fully automated image based data reconstruction scheme. A suite of traditional and deep learning computer vision algorithms are developed for identification of charge current quasi-elastic (CCQE) like muon and electron neutrino interactions using the MicroBooNE detector. Given a model of the MiniBooNE low energy excess as due to an enhancement of electron neutrino type events, this analysis predicts a combined statistical and systematic $3.8 \sigma$ low energy signal in $13.2 \times 10^{20}$ POT of MicroBooNE data. When interpreted in the context of $\nu_{\mu} \rightarrow \nu_{e} 3+1$ sterile neutrino oscillations a best fit point of $\left(\Delta m_{41}^{2}, \sin ^{2} 2 \theta_{e \mu}\right)=$ $(0.063,0.794)$ is found with a $90 \%$ confidence allowed region consistent with $>0.1 \mathrm{eV}^{2}$ oscillations. 


\section{Table of Contents}

List of Figures

List of Tables $\quad$ xxvi

\begin{tabular}{lll}
\hline & Introduction & 1
\end{tabular}

1.1 Overview of Issues Associated with Data Reconstruction in MicroBooNE . 2

1.2 Outline of Thesis . . . . . . . . . . . . . . . . . . 4

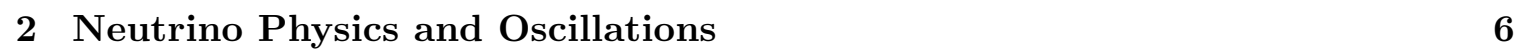

2.1 Introduction to Neutrinos . . . . . . . . . . . . . . . . . . . 6

2.2 Neutrino Oscillations . . . . . . . . . . . . . . . . . . . . . . 7

2.3 Sterile Neutrinos $\ldots \ldots \ldots \ldots \ldots \ldots$

\begin{tabular}{lll}
\hline & The MicroBooNE Detector & 12
\end{tabular}

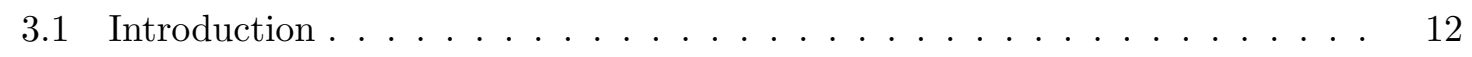

3.2 Time Projection Chamber . . . . . . . . . . . . . . . . . . . . . . 13

3.3 Optical System $\ldots \ldots \ldots \ldots \ldots$

3.4 Electronics Readout and Event Trigger . . . . . . . . . . . . . . . . 17

4 The Booster Neutrino Beam $\quad 21$

4.1 The Booster Proton Beam . . . . . . . . . . . . . . . . . . . . 21

4.2 Proton Target and Focusing Horn . . . . . . . . . . . . . . . . . . . 22

4.3 Beam Composition . . . . . . . . . . . . . . . . . . . . . 24 
5 Low Energy Excess: LSND and MiniBooNE 25

$5.1 \quad$ The LSND Signal $\ldots \ldots \ldots \ldots \ldots \ldots$

5.2 The MiniBooNE Experiment $\ldots \ldots \ldots \ldots \ldots \ldots$

5.2 .1 MiniBoonE Detector . . . . . . . . . . . . . . . . . . . . 27

5.2 .2 Event Selection . . . . . . . . . . . . . . . . . . . . 29

5.3 Oscillations Results $\ldots \ldots \ldots \ldots$. . . . . . . . . . . . . . . . . . 30

6 Low Energy Excess: MicroBooNE

6.1 MicroBooNE In Context . . . . . . . . . . . . . . . . . . . . . . . . . . 32

6.2 LEE Prediction in MicroBooNE . . . . . . . . . . . . . . . . . . . . . 34

6.2 .1 Unfolding MiniBooNE Data . . . . . . . . . . . . . . . . . . 34

6.2 .2 Unfolding Methodology . . . . . . . . . . . . . . . . . . 35

$6.2 .3 \quad$ Electron-like LEE Signal Model . . . . . . . . . . . . . . . . . . . . . 36

$\begin{array}{llll}7 & 1 & \text { Lepton } 1 & \text { Proton (1L1P) Neutrino Topology }\end{array}$

7.1 1L1P Signature . . . . . . . . . . . . . . . . . . . . . 41

7.1 .1 Motivation . . . . . . . . . . . . . . . . 41

7.1 .2 Topology $\ldots \ldots \ldots \ldots$. . . . . . . . . . . . . . . . . . . 42

7.1 .3 MicroBoonE Event Rate . . . . . . . . . . . . . . . . . . . 46

7.2 LEE Signal Prediction . . . . . . . . . . . . . . . . . . . 47

8 Image Based Neutrino Reconstruction $\quad 49$

8.1 Pixelized LArTPC Data Representation . . . . . . . . . . . . . . . . 50

8.2 Summary of Image Based Reconstruction $\ldots \ldots \ldots$. . . . . . . . . . . . . 52

8.3 PMT Precuts . . . . . . . . . . . . . . . . . . . . . 63

8.3 .1 Algorithm . . . . . . . . . . . . . . . 63

8.3 .2 Efficiency . . . . . . . . . . . . . . . . . 65

8.4 Cosmic Ray Tagging \& Contained Region of Interest . . . . . . . . . . . . . 67

$8.4 .1 \quad$ Boundary Crossing Reconstruction . . . . . . . . . . . . . . 67

8.4 .2 Through-Going Muon Reconstruction . . . . . . . . . . . . . . 69

$8.4 .3 \quad$ Stopping Muon Reconstruction . . . . . . . . . . . . . . . . . 71 
8.4 .4 Cosmic Pixel Tagging Efficiency _ . . . . . . . . . . . . . 73

8.4.5 Contained Region of Interest Reconstruction _ . . . . . . . . . . . 75

8.5 Track and Shower Pixel Identification using Semantic Segmentation . . . 76

$8.5 .1 \quad$ Network Architecture $\ldots \ldots \ldots \ldots$. . . . . . . . . . 76

8.5 .2 Network Training . . . . . . . . . . . . . . . . . . . . . 78

8.5 .3 Performance . . . . . . . . . . . . . . . 80

$8.6 \quad$ Vertex Finding \& Particle Clustering . . . . . . . . . . . . . . . . . . . . . 83

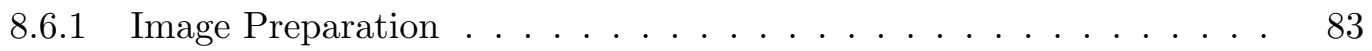

8.6 .2 Track Vertex Finding $\ldots \ldots \ldots \ldots$

8.6 .3 Track - Shower Vertex Finding . . . . . . . . . . . . . . . . . . . 92

8.6 .4 Particle Clustering . . . . . . . . . . . . . . . . 96

8.6 .5 Vertex Resolution . . . . . . . . . . . . . . . . . . . . 100

8.6 .6 Vertex Efficiency $\ldots \ldots \ldots \ldots$. . . . . . . . . . . . . . 101

$8.7 \quad 1 \mu 1 \mathrm{P}$ Reconstruction $\ldots \ldots \ldots \ldots$. . . . . . . . . . . . . . 109

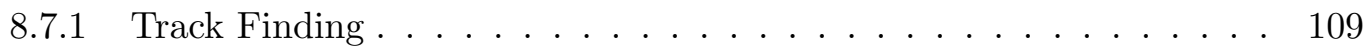

8.7 .2 Track Efficiency $\ldots \ldots \ldots \ldots$

8.7 .3 Angular Resolution . . . . . . . . . . . . . . . . . . . . . 113

8.7 .4 Energy Reconstruction . . . . . . . . . . . . . . . . . . . . . . 114

$8.7 .5 \mathrm{dE} / \mathrm{dx}$ Reconstruction $\ldots \ldots \ldots \ldots \ldots$

8.8 1e1P Reconstruction . . . . . . . . . . . . . . . . . . . . . . . . . 121

$8.8 .1 \quad$ Topological Features . . . . . . . . . . . . . . . . . . . . . . . . 121

$8.8 .2 \quad 1 e 1 \mathrm{P}$ Algorithm $\ldots \ldots \ldots \ldots \ldots \ldots \ldots$

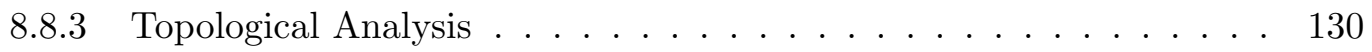

8.8 .4 Angular Reconstruction . . . . . . . . . . . . . . . . . . . 132

$8.8 .5 \quad$ Energy Reconstruction . . . . . . . . . . . . . . . . . . . . 134

$8.8 .6 \mathrm{dE} / \mathrm{dx}$ Reconstruction . . . . . . . . . . . . . . . 136

$8.8 .7 \quad$ Flash Matching . . . . . . . . . . . . . . . . . . . . . 138

$8.9 \quad$ Particle ID using a Convolutional Neural Network $\ldots \ldots$. . . . . . . . . . . . 139

8.9 .1 Network Architecture $\ldots \ldots \ldots$. . . . . . . . . . . . . 139

$8.9 .2 \quad$ Network Training . . . . . . . . . . . . . . . . . . . . . . 141 
8.9 .3 Performance $\ldots \ldots \ldots \ldots \ldots$

$\begin{array}{lll}9 & \text { Neutrino Event Selection } & 146\end{array}$

$9.1 \quad 1 \mu 1 \mathrm{P}$ Selection $\ldots \ldots \ldots \ldots \ldots \ldots \ldots \ldots$

9.1 .1 Overview . . . . . . . . . . . . . . . . . . . 147

$9.1 .2 \quad$ Pre-cuts . . . . . . . . . . . . . . . . . . . . . . . . 148

9.1 .3 Likelihood Selection . . . . . . . . . . . . . . . . . . . . . . 153

$9.1 .4 \quad$ Data-MC Comparison . . . . . . . . . . . . . . . . 157

$9.21 e 1 \mathrm{P}$ Selection $\ldots \ldots \ldots \ldots \ldots \ldots \ldots \ldots$

$9.2 .1 \quad$ Overview . . . . . . . . . . . . . . . . . . . 161

9.2 .2 Topological BDT $\ldots \ldots \ldots \ldots \ldots$

$9.2 .3 \quad$ Kinematic \& Multi-PID SVMs $\ldots \ldots \ldots \ldots$

9.2 .4 Combined Selection $\ldots \ldots \ldots \ldots$

$\begin{array}{ll}10 \text { Systematic Errors } & 181\end{array}$

10.1 Overview . . . . . . . . . . . . . . . . . . . . . . . . . . 181

10.2 GENIE Cross Section $\ldots \ldots \ldots \ldots$

10.3 BNB Flux $\ldots \ldots \ldots \ldots$

10.4 Detector Systematics $\ldots \ldots \ldots$. . . . . . . . . . . . . . 185

$10.5 \nu_{\mu}$ Constraint $\ldots \ldots \ldots \ldots \ldots \ldots \ldots \ldots$

$\begin{array}{ll}11 \text { Low Energy Excess Search } & 190\end{array}$

11.1 Significance of an LEE Signal . . . . . . . . . . . . . . . . . . . . . 190

11.2 Sensitivity to $3+1$ Oscillations $\ldots \ldots \ldots \ldots \ldots \ldots$

\begin{tabular}{ll}
\hline 12 Conclusions & 198
\end{tabular}

\begin{tabular}{ll}
\hline Bibliography & 201
\end{tabular}

\begin{tabular}{lll}
\hline I Appendices & 208
\end{tabular}

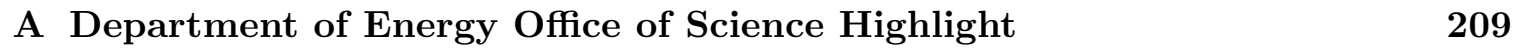


B Michel Electron Reconstruction $\quad 212$

B.1 Michel Electron Sideband . . . . . . . . . . . . . . . . . . . . . . . . 212

B.1.1 Motivation . . . . . . . . . . . . . . . . . . . 212

B.1.2 Selection . . . . . . . . . . . . . . . . . . 213

B.1.3 Data-MC Comparison . . . . . . . . . . . . . . . . 215 


\section{List of Figures}

1.1 MicroBooNE data event display featuring a candidate CCQE interaction with one lepton and one proton in the final state. Examples of cosmogenic backgrounds and detector effects such as TPC and PMT noise and the presence of dead wires complicate automated reconstruction of neutrino interactions. 3

2.1 Three neutrino mass spectrum for normal (left) and inverted (right) hierarchy. The lepton content of each mass eigenstate is denoted in color and represents the level of mixing with each weak eigenstate. . . . . . . . . . 9

2.2 Illustration of three neutrino mass states and their relationship with the fourth sterile neutrino mass splitting. . . . . . . . . . . . . . . . . . . . 10

$3.1 \quad$ Overview of the Fermilab experimental site showing the location of MicroBooNE along the BNB beam line. . . . . . . . . . . . . . . . . . 13

3.2 Left: Schematic of the MicroBooNE cryostat (outer cylinder). The top of the detector features feed through connections for interfacing warm and cold electronics. On side of the field cage can be seen within the cryostat. Right: Cross section of the MicroBooNE detector with notable components labelled. 14

3.3 Left: Image shows the cathode plane surrounded by the stainless steel tubes which comprise the field cage. Right: The three anode plane sense wires are shown. Insert shows the orientation of wires of the U, V, and Y planes. . . 14

\begin{tabular}{lll|l|l|l}
\hline 3.4 & LArTPC signal formation diagram described in Section & 3.2
\end{tabular} . . . . . . . 15 
3.5 Example neutrino candidate event observed in MicroBooNE. The same inter-

action is shown on the U, V, and Y wire planes. A candidate muon, proton, and two photons from $\pi^{0}$ decay are likely present as final state particles. . . 16

$3.6 \quad$ Left: Diagram of a MicroBooNE 8-inch PMT. Right: PMT rack array located behind the anode wire planes and mounted on the MicroBooNE cryostat wall. 17

3.7 MicroBooNE readout schematic. The cold electronics located within the detector are shown in the left including the TPC and PMT channels. Data are read out via cables through warm feedthroughs and the flange to racks of electronics located on the right. A trigger board hosts accelerator signals, and external triggering logic for detector readout. . . . . . . . . . . . . . 18

3.8 The measured distribution of flash times (requiring flashes greater than 50 PE) with respect to the trigger time for BNB-triggered events, shown as a ratio to the expected cosmic rate from off-beam data [1]. The blue band denoting the cosmic rate was centered at one, with a width corresponding to the measured uncertainty in the cosmic rate. A clear excess can be seen due to neutrinos between 3 and $5 \mu$ s after the trigger where neutrinos are expected. 20

4.1 Overhead view of the Fermilab accelerator complex surrounding the BNB. The MicroBooNE detector is located on the same beam line as the MiniBooNE detector. . . . . . . . . . . . . . . . . . . . . . . . 22

4.2 Left: BNB target enclosure. Right: Magnetic focusing horn schematic. The target rests inside within the inner cylindrical cavity (green). . . . . . . . . 23

4.3 Diagram of an incident proton beam on the target located within the magnetic focusing horn. Running in the neutrino mode, the horn selects $\pi^{+}$ particles for passage into a $50 \mathrm{~m}$ long decay region. The pions decay to a muon type particle and neutrino in the decay region. The muon type particle is absorbed in the dirt, while the neutrino travels onward in the beam direction. 23

4.4 Neutrino flux prediction at MicroBooNE for four neutrino states as modelled by the MiniBooNE beam simulation applied to MicroBooNE [2]. . . . . . . 24 
5.1 Observed LSND data (black points) above expected backgrounds (green and red) and the best fit spectrum for $\bar{\nu}_{\mu} \rightarrow \bar{\nu}_{e}$ oscillations [3] (blue). Left: Data as a function of the outgoing positron energy. Right: Spectrum as a function of the ratio between the distance traveled by the neutrino $L$, and $E_{\nu}$ the reconstructed neutrino energy. . . . . . . . . . . . . . . . 26

5.2 The MiniBooNE detector subterranean enclosure (left) showing the experimental hall layout. The PMT configuration inside the tank and in the veto region is show on the right. A human is shown for scale. . . . . . . . . . . 28

5.3 Patterns of Cherenkov light characteristic of muon, electron, and neutral pion type particles. The left column shows the light emission behavior along the particle's trajectory while the right column shows the pattern of light observed the photomultiplier array. . . . . . . . . . . . . . 28

5.4 MiniBooNE electron neutrino CCQE neutrino mode data for $12.84 \times 10^{20}$ POT data [4]. The stacked colored histogram estimates the background content shown with constrained systematic uncertainties. . . . . . . . . 30

5.5 MiniBooNE allowed regions for combined neutrino and antineutrino mode \begin{tabular}{|cc|c|}
\hline running [4]. A total of $12.84 \times 10^{20}$ POT and $11.27 \times 10^{20}$ POT data are \\
\hline
\end{tabular} collected for neutrino and antineutrino mode respectively. . . . . . . . . . 31

5.6 MiniBooNE event excess in neutrino and antineutrino mode for the full \begin{tabular}{|c|}
\hline dataset with statistical and systematic uncertainties [4]. The solid (dashed) \\
\hline
\end{tabular} curve shows the best fit to the neutrino and antineutrino mode data assuming two neutrino oscillations. . . . . . . . . . . . . . . . . . . 31

6.1 The MiniBooNE low energy excess (MiniBooNE data minus predicted background) compared to the absolutely normalized MiniBooNE MC-predicted background originating from intrinsic $\nu_{e}$ events. Statistical uncertainties are shown on the data and MC predictions as the black bars and gray shaded region respectively. . . . . . . . . . . . . . . . . 37 
6.2 Left: True neutrino distribution of intrinsic $\nu_{e}$ events before (blue) and after (green) reconstruction in MiniBooNE. The combined reconstruction and CCQE selection efficiency is shown in the bottom plot. No MiniBooNE MC events are simulated below $200 \mathrm{MeV}$. Right: The response matrix constructed from the MC truth and reconstructed energy variables. The z scale corresponds to the probability. . . . . . . . . . . . . . . . . . . . . . 38

6.3 Top: MiniBooNE unfolded intrinsic $\nu_{e}$ spectra (red) overlaid on the nominal predicted MC. The unfolded spectrum lies exclusively below $600 \mathrm{MeV}$ in true neutrino energy. Bottom: Ratio of the unfolded to the nominal intrinsic $\nu_{e}$ spectrum with statistical uncertainties only. . . . . . . . . . . . . . . . . . . 39

6.4 Combined ratio of the unfolded to the nominal intrinsic $\nu_{e}$ spectrum from D'Agostini's (purple) and SVD (black) unfolding techniques for the $12.84 \times$ $10^{20}$ POT MiniBooNE dataset. . . . . . . . . . . . . 39

6.5 The ratio of the MiniBooNE unfolded electron-like LEE model to the intrinsic $\nu_{e}$ event rate in MicroBooNE as a function of true neutrino energy. . . . . . 40

7.1 Neutrino charged current cross section per nucleon divided by neutrino energy as a function of true neutrino energy. In the low energy region below 600 $\mathrm{MeV}$, neutrinos primarily scatter through a quasi-elastic process. . . . . . . 42

$7.2 \mathrm{U}, \mathrm{V}$, and Y plane projections of 1L1P event topologies represented as 2D images. Top: Example of a 1e1P event in MicroBooNE, where a single proton and electron are produced. Bottom: Example of a $1 \mu 1 \mathrm{P}$ event, where a single muon and proton are produced. . . . . . . . . . . . . . . . . . 43

7.3 $\quad$ Proton range in liquid argon as a function of initial proton kinetic energy. A $60 \mathrm{MeV}$ proton in a 1e1P interaction traverses a distance of approximately 4 centimeters. . . . . . . . . . . . . . . . . . . . . . . . 44

7.4 Fraction of charge current events which feature a 1e0P (blue), 1e1P (green), or $1 e \mathrm{NP}$ (red) final state topology as a function of true neutrino energy. . . $\quad 45$

7.5 Fraction of 1e1P events per true neutrino energy bin which are CCQE (blue), MEC (orange), or other CC (green, red, purple, brown). . . . . . . . . . . . 46 
7.6 Muon (left) and electron (right) neutrino stacked event rate for $13.2 \times 10^{20}$ POT in MicroBooNE for neutrinos contained inside the active volume. The blue distribution shows the 1L1P event rate, the green and red distributions show the rate for neutral current and charge current non 1L1P events respec-

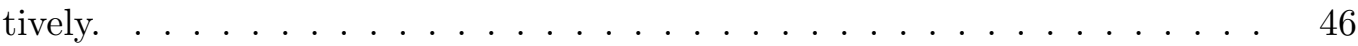

7.7 $\quad$ LEE signal model (gray) stacked on the 1e1P (blue), NC (green), and CC non 1e1P (red) rate expectations for 13.2e20 POT in MicroBooNE. . . . . . 47

7.8 The $1 e 1 \mathrm{P}$ component of the LEE signal (gray) stacked on the 1e1P (blue) rate expectation for $13.2 \times 10^{20}$ POT in MicroBooNE. . . . . . . . . . . . 48

8.1 Example output of the image based reconstruction chain after 3D vertexing finding for four different types of candidate vertices (yellow star). The neutrino interaction location is identified for $1 e 1 \mathrm{P}$ and $1 \mu 1 \mathrm{P}$ (top row) events along with a number of potential sources of backgrounds (bottom row) including false vertices found on cosmic rays and noise waveforms. The reconstructed particle clusters exiting the vertex are analyzed to determine consistency with electron, muon, and proton particles. . . . . . . . . . . 50

8.2 Example LArTPC data image of raw signal from MicroBooNE's collection plane. Cosmic muons are observed traversing the detector. Event comes from EXT-BNB trigger. No analysis selection is applied to select this event. 52

8.3 Three classes of pixel type reconstructed by the cosmic ray tagger. Throughgoing muons enter and exit the TPC boundary, stopping muons enter the TPC and stop inside, and contained objects whose cluster is completely contained within then TPC boundaries. . . . . . . . . . . . . . . . 54

8.4 Output of the cosmic ray tagging and cROI finding algorithm. Three objects are identified: through-going muon tracks (yellow), stopping muon tracks (pink), and a contained region of interest (blue) to search for a neutrino

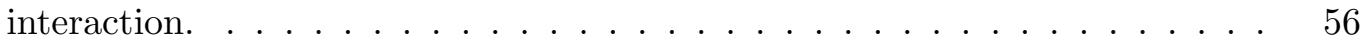


8.5 Example $1 \mu 1 \mathrm{P}$ and 1e1P SSNet input (top) and output (bottom). A LArCV image containing the particle ionization pattern, called an ADC image is used as input to SSNet. The network outputs an image of the same spatial dimension with track (yellow) and shower (cyan) pixels labelled. . . . . . . 58

$8.6 \quad$ Example of vertex finding applied to an example $1 \mu 1 \mathrm{P}$ (top) and $1 e 1 \mathrm{P}$ event (bottom). Top: A vertex is found (cyan star) close to the true vertex (yellow star) in the track only image (black pixels). Bottom: A track-vertex is found (green star) at the interface between track (yellow) and shower (cyan) pixels. 60

8.7 Tracking algorithm as applied to a $1 \mu 1 \mathrm{P}$ interaction. The left column shown the three planes event view. The right column shows the output of the tracking algorithm. Two tracks, green and red, have been reconstructed. . .

8.8 The front porch veto applied to a BNB-EXT event. The plot shows the total number of PE binned in 6 optical ticks ( $~ 90 \mathrm{~ns})$. A muon flash is identified in the pre-beam window which subsequently decays to an electron inside the beam window. . . . . . . . . . . . . . . . . . . . . . . 64

8.9 Efficiency tables for $\nu_{\mu}$ CCQE (left) and cosmic ray (right) events. The efficiency is optimized as a function of PMT maximum fraction and PE threshold and are selected (black box) for the given $\nu_{\mu}$ CCQE efficiency and cosmic ray rejection efficiency. . . . . . . . . . . . . . . . . . . . 65

8.10 Effect of applying the PMT precuts to samples of $\nu_{\mu}$ and $\nu_{e}$ CCQE events as a function of true neutrino energy. Approximately $97 \%$ efficiency is observed in both samples with little dependence on neutrino energy. . . . . . . . . . . 66

8.11 Ratio of the number of events passing both the PMT precut algorithm and the software trigger to the number of events passing the PMT precut algorithm for external unbiased data. . . . . . . . . . . . . . . . . . . . . 66

8.12 Example of a track (red line) crossing the top and bottom of the TPC. Top and bottom crossing points are identified by a coincident charge deposition on U, V, and Y wire plane. . . . . . . . . . . . . . . . 67 
8.13 An image of the Y-plane near the end of a stopping muon particle. The lowest purple line indicates the time of a cluster of PMT hits, called an optical flash. The upper line is the time of another optical flash plus the a single detector drift time. The track stops on the lower line and crosses the upper line. This track is consistent with entering or exiting the anode plane but not the cathode plane. . . . . . . . . . . . . . . . . . . . 68

8.14 Performance of the boundary point tagging algorithm for six types of detector boundaries. Left: Number of true (blue), and reconstructed (red) crossing points for each boundary type. Right: Ratio of the number of reconstructed to true crossing points. Top and bottom points are best reconstructed correctly while approximately half of anode crossing points are reconstructed. . $\quad 70$

8.15 Left: Fraction of true crossing muon end points tagged by the ThruMu tagger. On average, an MC event will have approximately $80 \%$ or more end points identified correctly. Right: The fraction of end points tagged versus the total number of pixels in the image. . . . . . . . . . . . . . . . . . 71

8.16 Example of ThruMu tracking applied to a simulated cosmic ray event. Crossing muon entry (red) and exit (blue) points are shown. Green lines represent the output of the ThruMu algorithm. . . . . . . . . . . . . . . . . . 72

8.17 An example MC intrinsic $\nu_{e}$ events with CORSIKA cosmic rays. ThruMu and StopMu tracks are shown as yellow and pink lines respectively. . . . . . 72

8.18 Distribution of cosmic tagged pixels for a sample of MCC8 intrinsic $\nu_{e}$ with CORSIKA cosmic rays. . . . . . . . . . . . . . . . . . . 73

8.19 Top: Example of a 1e1P interaction on the Y plane in false color scale with cosmic rays artificially removed. The right image shows the effect of the cosmic tagging algorithm (white pixels) applied to the event. Bottom: The distribution of fraction of neutrino pixels tagged as ThruMu for a sample of well reconstructed $1 e 1 p$ events. . . . . . . . . . . . . . . . . . . . . . . . . . 74

8.20 Example $1 \mu 1 \mathrm{P}$ collection plane event image featuring two cROI (blue boxes) placed at the mean position of the in-time flash (beige line). The cROI defines a sub region of the detector space for further pattern recognition. . . . . . . 75 
8.21 Efficiency of placing cROI on the event image consistent with the true neutrino vertex location as a function of true neutrino energy. The blue and red curves show the efficiency for placing the cROI on the image covering the neutrino vertex in at least two planes, and requiring one of them to be the collection plane respectively. The green curve shows the efficiency for placing the cROI on the image covering the vertex in all three TPC planes. . . . . . 76

8.22 Diagram of U-ResNet architecture. A 512 × 512 single channel image is input. Black arrows describe the direction of tensor data flow. Dashed green \begin{tabular}{l}
\hline lines indicate concatenation operations to combine filter outputs from the \\
\begin{tabular}{|c|}
\hline convolution layers to the input of deconvolution layers. The final output has \\
\hline the same spatial dimension as the input except for the depth which becomes \\
\hline
\end{tabular}
\end{tabular} 3 channels, one per pixel class . . . . . . . . . . . . . . . . . 77

8.23 Visualization of example input and output data tensors for a $1 e 1 \mathrm{P}$ interaction. The left column shows the input LArCV image (top) and the true pixel-wise label (bottom) used to train the SSNet. The labeled image shows track pixels in yellow, and shower pixels in cyan. The right column shows the network output which is a pixel-wise normalized score of being a background (top), shower (middle), and track (bottom) type. . . . . . . . . . . 78

8.24 Top: An example LArCV image from the training set in which two protons, \begin{tabular}{|l|}
\hline one electron, and one muon are produced. Bottom: The event from the top \\
\hline image that shows the categories for pixel-wire loss weighting indicated in \\
\hline
\end{tabular} different colors. . . . . . . . . . . . . . . . . . . 80

8.25 (a) The loss value as a function of training time using the validation sample. The red line shows the average at a given epoch using 200 the neighboring points. (b) Incorrectly Classified Pixel Fraction (ICPF) as a function of training time using the validation sample. The drop in both plots at epoch 14 is due to lowering of the learning rate by a factor of $10 .$. . . . . . . . . 81 
8.26 ICPF error rates for labelling proton (track) and electron (shower) pixels for the test sample versus kinematic variables from simulation. (a) The electron kinetic energy from the 1e1P-LE sample. (b) The proton kinetic energy from the 1e1P-LE sample. (c) The opening angle between two particles from the

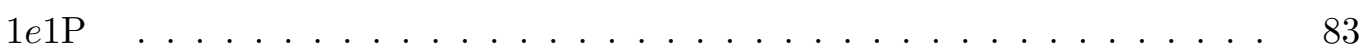

8.27 Three separate images are provided as input into the vertex finding algorithm. An ADC image which contains all pixels in the cROI. A track image which contains only track labelled pixels. A shower image containing only shower labelled pixels. Example inputs are shown for a $1 e 1 \mathrm{P}$ (top row) and $1 \mu 1 \mathrm{P}$ (bottom row) interaction. . . . . . . . . . . . . . . . . 84

8.28 Left: Example of a star shaped object containing 6 obvious prongs. Right: Output of the algorithm. A collection of 6 straight clusters connected at a central point, or a potential vertex seed. . . . . . . . . . . 85

8.29 Algorithm steps 1 - 5, described in Section 8.6 .2 , for analyzing a $1 \mu 1 \mathrm{P}$ interaction to locate vertex seeds. . . . . . . . . . . . . . . . . . 86

8.30 Distinction between low (left) and high (right) charge regimes for a $1 \mu 1 \mathrm{P}$ interaction. The low charge regime encapsulates all pixels in the image above 10 ADC. The high charge regime contains pixels above a higher charge threshold. In this example, only the proton bragg peak passes the HC threshold. . 87

8.31 Algorithm steps $6 \& 7$, described in Section 8.6 .2 , for analyzing a $1 \mu 1 \mathrm{P}$ interaction to locate vertex seeds. . . . . . . . . . . . . . . . . 88

\begin{tabular}{|ll|l|l|}
\hline 8.32 & Algorithm steps a-d, described in Section & 8.6 .2 , for analyzing a $1 \mu 1 \mathrm{P}$ inter- \\
\hline
\end{tabular} action to locate the $3 \mathrm{D}$ vertex from vertex seeds. . . . . . . . . . . . . . . 89

8.33 Procedure for scanning the collection of vertex seeds to search for the best kink location by minimizing $\mathrm{d} \Theta$ as described in the text. . . . . . . . . . . . 91

8.34 Top: Minimization of $\mathrm{d} \Theta$ performed for vertex seeds in each of the three wire planes. Bottom: Sum of $\mathrm{d} \Theta$ for coincident time tick and wire. A local minimum is found which defines a 3D consistent kink. . . . . . . . . . . . 92

8.35 Reconstructed 3D vertex position (cyan) and true vertex position (yellow) for the example $1 \mu 1 \mathrm{P}$ interaction. . . . . . . . . . . . . . . 93 
8.36 Example SSNet images of a 1e1P interaction to demonstrate the track-shower vertex finding algorithm. Track and shower pixels are labelled as yellow and cyan respectively. . . . . . . . . . . . . . . . . . . . . . . . . . 93

8.37 Algorithm step, described in Section 8.6 .3 , used to locate a consistent 3D edge where a shower is attached to a track. . . . . . . . . . . . . . . . . 94

8.38 The grid scan procedure for locating the best 3D interaction vertex for trackshower events. Steps are described in Section 8.6 .3 . . . . . . . . . . . . . 95

8.39 Location of the reconstructed 3D vertex (green star) for the example 1e1P

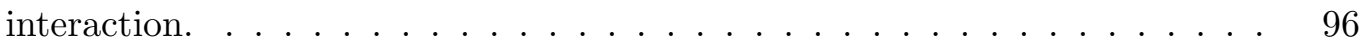

8.40 Particle clustering steps a-c for a $1 \mu 1 \mathrm{P}$ interaction as described in Section

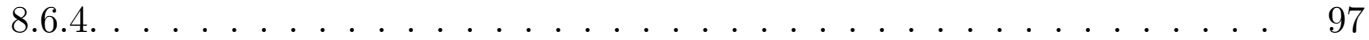

8.41 Particle clustering steps using a polar coordinate system described in Section

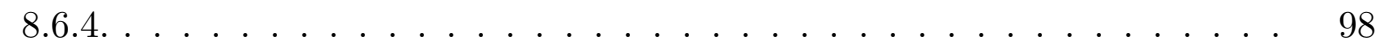

8.42 Particle clustering steps to connect parent clusters found inside the circle to clusters found outside. Two unique clusters are identified for the example $1 \mu 1 \mathrm{P}$ interaction. . . . . . . . . . . . . . . . . . . 99

8.43 Example of the cluster matching procedure applied to a simulated neutrino event and described in Section 8 8.6.4 . . . . . . . . . . . . . . . . . . 100

8.44 Distribution of vertex resolution $(\Delta \mathrm{R})$ for $1 \mu 1 \mathrm{P}$ (left) and $1 e 1 \mathrm{P}$ (right) events. 101

8.45 True neutrino energy distribution are shown for $1 \mu 1 \mathrm{P}$ (left) and $1 e 1 \mathrm{P}$ (right) events. . . . . . . . . . . . . . . . . . . . . 102

8.46 Good Event efficiency as a function of true neutrino energy for $1 \mu 1 \mathrm{P}$ (left) and 1e1P (right) events. Approximately $90 \%$ of events feature the interaction vertex inside a cROI and at least 5 pixels away from dead wires. . . . . . . 102

8.47 Spatial distribution of the Good Event efficiency binned in the Y-Z detector space. . . . . . . . . . . . . . . . . . . . 103

8.48 Vertexing efficiency as a function of true neutrino energy for $1 \mu 1 \mathrm{P}$ (left) and 1e1P (right) Good Events. The upper line in each plots shows the vertexing efficiency for perfect pixel labelling and the lower line shows the efficiency for using the SSNet pixel label. . . . . . . . . . . . . . . . . . . . . . . . . . 104 
8.49 A 1e1P interaction with Perfect Segment (left), and SSNet (right). Track and shower pixel are labelled as yellow and cyan respectively. A zoom of the vertex region is shown in the bottom portion of the figure. A mis-classification of electron pixels as track in the vertex region removes the presence of a shower on track edge feature. . . . . . . . . . . . . . . . . . . . 105

8.50 Vertexing efficiency for $1 \mu 1 \mathrm{P}$ (left) and $1 e 1 \mathrm{P}$ Good Events (right) as a function of true neutrino energy. The top line in each plot represents the vertexing efficiency for Perfect Segment. The bottom line represents turning on the ThruMu cosmic ray tagging algorithm. . . . . . . . . . . . . . 106

8.51 Opening angle distribution between the lepton and proton for $1 \mu 1 \mathrm{P}$ (left) and $1 e 1 \mathrm{P}$ (right) events. . . . . . . . . . . . . . . 106

8.52 Proton length distribution for $1 \mu 1 \mathrm{P}$ (left) and $1 e 1 \mathrm{P}$ (right) events. . . . . . 107

8.53 Vertex efficiency as a function of lepton and proton opening angle for $1 \mu 1 \mathrm{P}$ (left) and 1e1P events (right). The highest efficiency is achieved when the opening angle is near 90 degrees. . . . . . . . . . . . . . . . . . 107

8.54 Vertexing efficiency as a function of proton length projected on to the $\mathrm{Y}$ plane image for $1 \mu 1 \mathrm{P}$ (left) and 1e1P (right) events. The efficiency decreases as the proton decreases in size. . . . . . . . . . . . . . . . . . 108

8.55 Cumulative vertexing efficiency as a function of true neutrino energy for $1 \mu 1 \mathrm{P}$ (left) and $1 e 1 \mathrm{P}$ (right) events. . . . . . . . . . . . . . . . . 109 
8.56 (a) Dots represent the set of 3D points in the proto-track. The black dots are the points that have been already sorted, and the light blue dots are the remaining unsorted points. The green and red dots correspond to the vertex and end of track respectively. The end point is selected as the 3D point the furthest away from the vertex. The red line corresponds to the path found within the sorted 3D points. For each unsorted point (dark blue point) the two lengths and angles are computed : $L_{1}$ is the distance to the last selected 3D point, $L_{2}$ is the distance to the end of the proto-track, $\theta$ is the angle from the last two sorted points to the candidate, and $\phi$ is the angle between the candidate, the last sorted point, and the end of the proto-track. (b) Tracking algorithm following a track using the stochastic search procedure. . . . . . . 112

8.57 Tracking efficiency as a function of kinetic energy for proton (left) and muon (right). . . . . . . . . . . . . . . . . . . . 113

8.58 Tracking efficiency as a function of true neutrino energy for combined proton and muon track reconstruction. . . . . . . . . . . . . . . . . . . . . . . 114

8.59 Track angle definition for $\phi$ and $\theta$ with respect to the MicroBooNE detector geometry. . . . . . . . . . . . . . . . . . . . . . . 114

8.60 Error on the reconstruction of $\phi$ for protons (left) and muons (right). The red line corresponds to a Gaussian fit of the central peak. A resolution of $(7.9 \pm 0.2)$ deg. for protons and $(4.1 \pm 0.1)$ deg. for muons is achieved. The inserts in the upper right hand portion of the plot show the track by track correlation between the reconstructed and the true $\phi$ angle. . . . . . . . . . 115

8.61 Error on the reconstruction of $\theta$ for protons left) and muons (right). The red line corresponds to a Gaussian fit of the central peak. A resolution of $(4.2 \pm 0.1)$ deg. for protons and $(3.5 \pm 0.1)$ deg. for muons is achieved. The inserts in the upper right hand portion of the plot show the track by track correlation between the reconstructed and the true $\theta$ angle. . . . . . . . . . 115

8.62 Kinetic energy as a function of range in liquid argon (LAr) for proton (blue) and muon $(\mathrm{red})$ particles. . . . . . . . . . . . . . . . . . 116 
8.63 Relative differnce between the reconstructed energy and the true energy as a function of the true kinetic energy at the single-track level proton (left) and muon (right) tracks. . . . . . . . . . . . . . . . . . . . 116

8.64 Left: Comparison of the reconstructed energy to the true MC energy. Right: Evolution of the resolution as a function of the true energy. . . . . . . . . . 117

8.65 Average pixel intensity along each reconstructed track defined in Equation|8.5. The red and blue distributions represent the tracks with the highest (proton like) and lowest (muon like) average ionization. . . . . . . . . . . . . . 118

8.66 Estimation of the recombination parameters for the modified box model. The 2D histogram shows for each reconstructed 3D point over a sample of proton tracks, the pixel intensity as a function of the expected $d E / d x$. The black dots correspond to the average of the distribution for each slice in expected $d E / d x$. The blue line represents the expected profile, and the red line is a fit of the model to the black dots. . . . . . . . . . . . . . . . . . . . . . 119

8.67 Distributions of reconstructed $d E / d x$ as a function of residual length for proton (left) and muon (right) tracks. The black and red lines respectively show the expected muon and proton $d E / d x$ versus residual length curves. . 120

8.68 Correction factor as a function of the Y-Z detector space defined as the ratio of $d Q / d x$ in each divided by the global average $d Q / d x$. . . . . . . . . . . 121

8.69 Energy loss for electrons as a function of initial kinetic energy. . . . . . . . 122

8.70 True shower deposited energy distribution for $<600 \mathrm{MeV} 1 e 1 \mathrm{P}$ interactions. Event displays from five energy bins are shown to illustrate the electron features described in Section 18.8 .1 . . . . . . . . . . . . . . . . . . . . 123

8.71 True proton deposited energy distribution for $<600 \mathrm{MeV} 1 e 1 \mathrm{P}$ interactions. Event displays from three energy bins are shown to illustrate the proton features described in Section

8.72 Left: Distribution of electron (red) and proton (blue) true particle length as projected onto the Y plane for sub $800 \mathrm{MeV} 1$ e1P interactions. Right: A 370 $\mathrm{MeV} 1 e 1 \mathrm{P}$ interaction with $\mathrm{X}$ and $\mathrm{Y}$ pixel scale. The charge deposition fits in a $400 \times 400$ pixel window centered around the vertex. . . . . . . . . . . 126 
8.73 An example input into the $1 e 1 \mathrm{P}$ algorithm showing a $400 \mathrm{x} 400$ pixel crop around the vertex in each of the three planes. . . . . . . . . . . . . . . 126

8.74 Steps for identifying cosmic ray tracks within the image crop described in Section $\mid 8.8 .2 \ldots \ldots \ldots \ldots \ldots \ldots \ldots$

8.75 Examples of the cosmic ray tagging algorithm applied to six reconstructed \begin{tabular}{|cc|}
\hline vertices. Top Row: Cosmic algorithm crosses a proton particle of a $1 e 1 \mathrm{P}$ \\
\hline
\end{tabular} interaction. Cosmic algorithm crosses the trunk of an electron particle. Cosmic crosses both the electron and proton of a $1 e 1 \mathrm{P}$ interaction. Bottom Row: Cosmic algorithm follows a stopping muon until the track bends too severely, stopping before the true end point. A stopping muon is completely contained inside the image and not tagged by the cosmic algorithm. Cosmic tagger touches a $1 e 1 \mathrm{P}$ interaction vertex. . . . . . . . . . . . . . . 129

8.76 Steps $4-6$ of the $1 e 1 \mathrm{P}$ algorithm as described in Section 8.8 .2 . . . . . . . . 130

\begin{tabular}{lll|l}
\hline 8.77 The final step of the $1 e 1 \mathrm{P}$ algorithm as described in Section & 8.8 .2 . Two
\end{tabular} particles have been reconstructed and matched across planes. . . . . . . . . 131

8.78 Topologically reconstructed parameters for electron and proton particles using the $1 e 1 \mathrm{P}$ algorithm described in Section 8.8 .3 . . . . . . . . . . . . . . 132

8.79 Right: Reconstructed versus true proton angle with respect to the beam for a sample of $1 e 1 \mathrm{P}$ events. The magenta line shows $y=x$ to characterize the bias. Left: Fractional difference between the reconstructed and true angle with a double gaussian fit. The angular resolution is approximately 4\%. . . 133

8.80 Right: Reconstructed versus true electron angle with respect to the beam for a sample of $1 e 1 \mathrm{P}$ events. The magenta line shows $y=x$ to characterize the bias. Left: Fractional difference between the reconstructed and true angle with a double gaussian fit. The angular resolution is approximately 6\%. . . 134

8.81 Proton (top) and electron (bottom) summed collection plane pixel value versus true deposited energy for low energy 1e1P events. In each true energy bin a gaussian is fit to the pixel value distribution to estimate the mean and spread (blue points). A linear fit (magenta line) is optimized for the blue

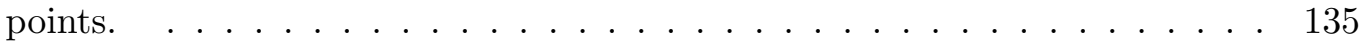


8.82 Fractional energy resolution for proton (left) and electron (right) particles as a function of true deposited energy. An approximately $10 \%$ and 20\% energy resolutions are observed for proton and electron particles respectively using calorimetry . . . . . . . . . . . . . . . . . . 136

8.83 Left: $d Q / d x$ profiles as a function of particle length for proton (blue) and electron (red). The dotted line represents the binned charge deposition per length and the solid line is a truncated mean for smoothing. Right: The $1 e 1 \mathrm{P}$ events corresponding to the $d Q / d x$ profiles shown on the left. . . . . . 137

8.84 Electron (blue) and proton (red) median $d E / d x$ value for a simulated sample of $1 e 1 \mathrm{P}$ events. A median of $\sim 2.2 \mathrm{MeV} / \mathrm{cm}$ and $\sim 5 \mathrm{MeV} / \mathrm{cm}$ is observed. . 137

8.85 Proton $d E / d x$ as a function of residual range. An increase in charge deposition per unit length is observed toward the end of a proton's track. . . . . . 138

8.86 Cartoon of a flash hypothesis is constructed of a $1 \mu 1 \mathrm{P}$ interaction. A binned $d E / d x$ is reconstructed for the muon particle. Each charge deposition is converted to a light yield using the Photon Library forming a light hypothesis per PMT. . . . . . . . . . . . . . . . . . . . . . 139

8.87 Example flash match for a 1e1P event. Left: The hypothesis (blue) spectrum is compared to the reconstructed flash (black) and a shape only $\chi^{2}$ is formed. Right: Distribution of shape only $c h i^{2}$ for a simulated sample of $1 e 1 \mathrm{P}$ events. 140

8.88 Multi-PID network architecture described in Section 8.9.1] . . . . . . . . . 141

8.89 Network training for U (upper left), V (upper right), and Y (bottom) plane. The blue and red curves show the accuracy on the train and test samples respectively. The gold curve shows the loss value as a function of time. . . . 142

8.90 PID score distributions from the Multi-PID network for five particle types for $1 e 1 \mathrm{P}$ (left) and $1 \mu 1 \mathrm{P}$ (right). . . . . . . . . . . . . . . . 143 
9.1 The $1 e 1 \mathrm{P}$ stacked background prediction as a function of reconstructed neutrino energy for estimating the $5 \times 10^{19}$ POT open data set before selection as described in Chapter 9 , Black dots represents Run 1 detector data. The gray boxes represent MC statistical uncertainty. The cosmic ray contamination (yellow and orange distributions) for the 1e1P search is high. Selection is applied in this chapter to reduce cosmic and neutrino induced backgrounds. Other distributions are described in the text. Data are shown in linear scale (left) and logarithmic scale (right). . . . . . . . . . . . . . . . 147

9.2 Schematic of single-transverse kinematic imbalance [5]. . . . . . . . . . . 151

\begin{tabular}{|lll|l|l|}
\hline 9.3 & Transverse momentum variables $\delta p_{\mathrm{T}}$ and $\delta \phi_{\mathrm{T}}$ described in Section & 9.1 .2 & \\
\hline
\end{tabular} Three distributions are shown for $1 \mu 1 \mathrm{P}$ events (blue), EXT-BNB events (green), and neutrino backgrounds events (red). Black dashed line indicates the location of the cut value. . . . . . . . . . . . . . . . . . . . . 151

9.4 Four momentum transfer, $Q^{2}$, distribution for $1 \mu 1 \mathrm{P}$ events (blue), EXTBNB events (green), and neutrino background events (red). Black dashed line indicates the location of the cut value. . . . . . . . . . . . . . . . . 152

9.5 Charge deposition per unit length asymmetry, $\eta$, between the proton and

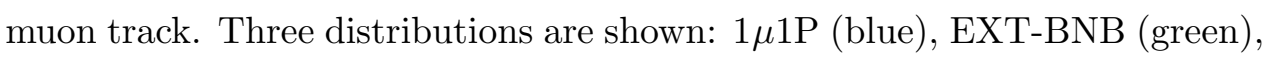
and neutrino background $(\mathrm{red}) . \ldots \ldots \ldots$. . . . . . . . . . . 154

9.6 Track kinematic variables described in Section $\mid 9.1 .3$. Three distributions are

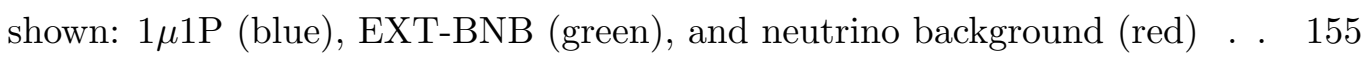

9.7 Bjorken scaling factor $\mathrm{x}$ for three samples: $1 \mu 1 \mathrm{P}$ (blue), EXT-BNB (green), and neutrino background $(\mathrm{red}) . \ldots \ldots \ldots \ldots$. . . . . . . . . . 156

9.8 Log-likelihood distributions for discriminating $1 \mu 1 \mathrm{P}$ events against cosmic rays (left) and neutrino backgrounds (right). The black dashed line represent the LL cut value to categorize a signal versus background. . . . . . . . . . . 156 
$9.9 \quad 1 \mu 1 \mathrm{P}$ selection efficiency as a function of true neutrino energy for interactions located in the active volume. A downward trend in efficiency is observed above $800 \mathrm{MeV}$ as muons reach large enough energy to exit the detector. A maximum efficiency of approximately $20 \%$ is achieved in the sub $600 \mathrm{MeV}$ region. . . . . . . . . . . . . . . . . . . 157

9.10 Cosmic (top) and Neutrino (bottom) LL score distribution absolutely normalized to $4 \times 10^{19}$ POT for the $1 \mu 1 \mathrm{P}$ selection. The data and prediction are shown with black and gray dots respectively with statistical uncertainty only. The predicted likelihood distributions are broken down by neutrino interaction type. The ratio of data to prediction per bin is shown in the lower plot. . . . . . . . . . . . . . . . . . . 159

9.11 Proton (top) and Muon (bottom) angle with respect to the beam absolutely normalized to $4 \times 10^{19}$ POT for the $1 \mu 1 \mathrm{P}$ selection. The data and prediction are shown with black and gray dots respectively with statistical uncertainty only. The predicted $\cos \theta$ distributions are broken down by neutrino interaction type. The ratio of data to prediction per bin is shown in the lower plot. . . . . . . . . . . . . . . . . . . 160

9.12 Reconstructed neutrino energy from the range based formula absolutely normalized to $4 \times 10^{19} \mathrm{POT}$ for the $1 \mu 1 \mathrm{P}$ selection. The data and prediction are shown with black and gray dots respectively with statistical uncertainty only. The predicted range based energy distribution is broken down by neutrino interaction type. The ratio of data to prediction per bin is shown in the lower plot. . . . . . . . . . . . . . . . . . . . 161

9.13 Four cosmic ray rejection precuts described in the text for topologically rejecting cosmic ray muons at the vertex location. . . . . . . . . . . . . 163

9.14 Number of particles reconstructed using the 1e1P algorithm for four samples. 164

9.15 Left: Distribution of line fraction for true electron and proton particles for sub $600 \mathrm{MeV} 1 e 1 \mathrm{P}$ interactions. Right: Example estimation of the line fraction by drawing a line of fixed thickness (cyan) for a proton cluster (red). . . . . 165 
9.16 Distribution of fractional difference between the CCQE and visible energy of the interaction for 1e1P events (blue) and EXT-BNB events (red). Magenta \begin{tabular}{|c|}
\hline lines represent the inner $68 \%$ of $1 e 1 \mathrm{P}$ events. $80 \%$ of EXT-BNB events lie \\
\hline
\end{tabular} outside the magenta region. . . . . . . . . . . . . . . 166

9.17 SSNet shower fraction for electron (top right) and proton (bottom right) particles reconstructed in $1 e 1 \mathrm{P}, \mathrm{CC} \nu \mu$, and EXT-BNB events. An example SSNet image of a $1 e 1 \mathrm{P}$ interactions is shown on the left where an electron

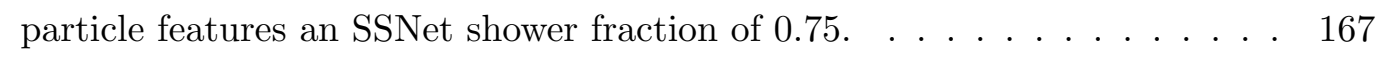

9.18 Left: Distribution of ratio of electron shower length to width for $1 e 1 \mathrm{P}$ events (blue) and neutrino backgrounds events (red). Right: Distribution of empty area ratio for electron particles for $1 e 1 \mathrm{P}$ events (blue) and neutrino background events (red) . . . . . . . . . . . . . . . . . 168

9.19 Discriminators used to identify electron topologies described in the text. Left: Distribution of number of branches for electron particles identified in $1 e 1 \mathrm{P}$ (blue) and neutrino background (red) events. Right: Distribution of number of defect points larger than 5 pixels. . . . . . . . . . . . . . . 169

9.20 Number of identified clusters emitted in the electron direction above $25 \mathrm{MeV}$ for 1e1P (blue) and neutrino background (red) events. . . . . . . . . . . 170

9.21 Score distributions for Cosmic BDT (left) and Neutrino BDT (right) for signal and background inputs. . . . . . . . . . . . . . 171

9.22 Importance of features used in the Cosmic (left) and Neutrino (right) BDTs. Feature importance is a normalized measure of the fraction of algorithm composed of the specified discriminant. . . . . . . . . . . . . . . . . 172

9.23 Event rate prediction for Cosmic (right) and Neutrino (left) BDT for backgrounds for the 1e1P selection described in the text. Run 1 data are shown as the black dot. . . . . . . . . . . . . . . . . . . . . 172

9.24 Event rate prediction as a function of reconstructed neutrino energy for an

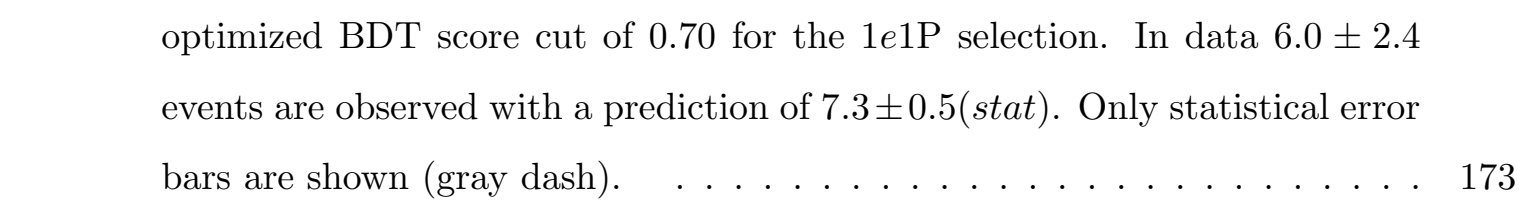


9.25 Reconstructed 1e1P candidate interaction identified in the Run 1 data set. The reconstructed energy is $166 \mathrm{MeV}$. . . . . . . . . . . . . . . . . 174

9.26 Reconstructed 1e1P candidate interaction identified in the Run 1 data set. The reconstructed energy is $1.1 \mathrm{GeV} . \ldots \ldots \ldots$. . . . . . . . 174

$9.271 e 1 \mathrm{P}$ efficiency as a function of true neutrino energy for 3 analysis cuts in the BDT based selection. The top line (green) is the cumulative vertex efficiency before selection cuts and represents the nominal output of the vertexing algorithm with backgrounds shown in Fig. $\mid 9.1$. The black, maroon, and purple lines represent the cosmic rejection cuts, and the Cosmic and Neutrino BDT cuts respectively. The stacked backgrounds compared to $5 \times 10^{19}$ POT data are shown in Fig. 9.24|after the Neutrino BDT cut. . . . . . . . . . . . . . . 175

9.28 Kinematic SVM classifier score applied to $1 e 1 \mathrm{P}$ (green) and $\nu_{\mu}$ (blue) background events. . . . . . . . . . . . . . . . . 177

9.29 Proton (upper left), muon (upper right), and electron (lower middle) score distributions. The blue and green stacked histograms are the Cosmic and $\nu_{\mu}$ backgrounds respectively. The black dots are the score distributions for the Run 1 data set. Good agreement is observed between data and prediction. . 178

9.30 Multi-PID SVM classifier applied to $1 e 1 \mathrm{P}$ (green) and $\nu_{\mu}$ (blue) events. . . 178

9.31 Combined classifier for $1 e 1 \mathrm{P}$ selection using the Topological BDT, the Kinematic SVM, and the Multi-PID SVM. Two distributions are shown: 1e1P (green) and $\nu_{\mu}$ (blue) interactions. . . . . . . . . . . . . . . 179

9.32 Reconstructed energy distribution for intrinsic $\nu_{e}$ events after the combined classifier. MC statistical error bars are shown. No other neutrino or cosmic backgrounds pass the combined selection. . . . . . . . . . . . . . 180

10.1 Left: Correlation matrix between $1 e 1 \mathrm{P}$ and $1 \mu 1 \mathrm{P}$ reconstructed energy bins for flux and cross section systematic variations. Right: Fractional covariance matrix for the same reconstructed energy binning. $\ldots \ldots \ldots$. . . . . . . . 187

10.2 Reconstructed energy spectrum scaled to $13.2 \times 10^{20}$ POT for the $1 e 1 \mathrm{P}$ (left) and $1 \mu 1 \mathrm{P}$ (right) selections including statistical and systematic errors. . . . 189 
11.1 Reconstructed energy spectrum for the predicted LEE signal (green) on the intrinsic $\nu_{e}$ only (blue) background for $13.2 \times 10^{20}$ POT. Statistical and systematic uncertainties are shown. . . . . . . . . . . . . . . . . . . . . 191

11.2 Frequentist study for rejecting the intrinsic $\nu_{e}$ only background hypothesis in $13.2 \times 10^{20}$ POT as described in Section $\mid 11.1$, The red and blue distributions represent the PDF of the $\chi^{2}$ test statistic under the background only and background plus LEE signal respectively. . . . . . . . . . . . . . . . . . . . 192

11.3 Correlation matrix between four samples: intrinsic $\nu_{e}$, the LEE signal, the full osc sample, and the intrinsic $\nu_{\mu}$ background. Strong correlations are observed between energy bins in each sample. . . . . . . . . . . . . . . . . . 194

11.4 One sided raster scan to test for sensitivity to $\Delta m_{41}^{2}$ oscillations as described in the text. . . . . . . . . . . . . . . . . . . . . . 195

11.5 Oscillations fit for $\nu_{e}$ appearance applied to the LEE signal as described in the text. The best fit point is shown as the red star. Various confidence levels are shown using the $\Delta \chi^{2}$ prescription. The allowed regions can be compared to the MiniBooNE result in Fig. 5.50 . . . . . . . . . . . . . . . . . . . . . . 196

11.6 The intrinsic $\nu_{e}$ background and low energy excess reconstructed energy prediction (green) compared to the best fit $\nu_{\mu} \rightarrow \nu_{e}$ oscillation spectrum (red).

B.1 Reconstructed Michel energy in $\mathrm{MeV}$ scale for the pixel based scheme (blue) applied to externally triggered data as described in Section|B.1. The energy spectrum from the hit based identification scheme from is overlaid in grey. . 214

B.2 Left: Reconstructed $d E / d x$ distribution for selecting Michel events in external data. The peak position lies in the expected MIP region. Right: Reconstructed opening angle between the muon and michel particle. . . . . 215

B.3 Left: Reconstructed Michel spectrum (blue) for the selection applied to simulated michel decays. The red distribution shows the true michel energy deposited energy. Right: Correlation between the reconstructed and true michel energy. The red dashed line is shown at $y=x$ to guide the eye. . . . 216

B.4 Absolutely normalized Michel spectrum compared to the Run 1 data set. Good agreement is observed between data and prediction. . . . . . . . . . . 216 


\section{List of Tables}

2.1 Neutrino oscillation parameters as of 2018 provided by the Particle Data Group $\left.{ }^{6}\right] . \ldots \ldots \ldots \ldots \ldots \ldots$

8.1 Values of the four metrics defined in Section 8.5 .3 to benchmark the SSNet

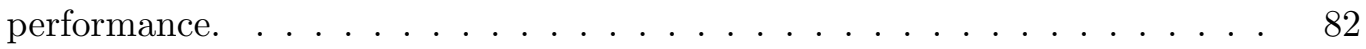

8.2 Table of relative efficiency for 5 components which contribute to the vertexing efficiency. The cumulative, or expected efficiency, for $1 \mu 1 \mathrm{P}$ and $1 e 1 \mathrm{P}$ events is shown in the bottom row . . . . . . . . . . . . . . . . . . . . . . . . . 109

8.3 $\quad$ Single particle ID performance for high resolution LArTPC images for three CNN networks. AlexNet and GoogLeNet results reproduced from the reference while VGG16b results are new. . . . . . . . . . . . . . . . . . . . . . . 140

8.4 Multi-PID network classification accuracy for low multiplicity electron neutrino interactions for a given topology. Values reported represent the fraction of events. . . . . . . . . . . . . . . . . . . . . . . . . . 144

8.5 Multi-PID network classification accuracy for low multiplicity muon neutrino interactions for a given topology. Values are reported represent the fraction of events. . . . . . . . . . . . . . . . . . . . . . 145

\begin{tabular}{|lll|l|l|l}
9.1 & $1 \mu 1 \mathrm{P}$ selection efficiency for stages of cuts described in Section & 9.1 .3
\end{tabular} . . . . 158

10.1 GENIE neutrino model parameters considered for cross section systematics. 184

10.2 Fractional uncertainties per $\nu_{e}$ energy bin before and after applying the constraint. Flux and cross section systematic uncertainties are constrained from approximately $25 \%$ to $10 \%$. . . . . . . . . . . . . . . . . 188 
12.1 Reconstructed neutrino energy bin-by-bin event rate expectation for the intrinsic $\nu_{e}$ background and electron-like LEE signal for $13.2 \times 10^{20}$ POT corresponding to Fig. 11.1. . . . . . . . . . . . . . . . . . . . . . . 200

12.2 Low energy excess signal significance for frequentist studies with statistical and systematic errors included. The nominal significance of $3.8 \sigma$ is shown at $5 \% 1 e 1 \mathrm{P}$ efficiency as described in this thesis. An efficiency improvement of $50 \%$ and $100 \%$ over the nominal result would improve the median LEE significance. The right most column shows the $1 \sigma$ downward fluctuation of the LEE significance. . . . . . . . . . . . . . . . . . . . . 200 


\section{Acknowledgments}

I first want to thank Mike Shaevitz for being an exceptional mentor. Thank you for your support, guidence, wisdom, and open-mindedness throughout my Ph.D.

A huge thanks goes to my wife, Ashley, for supporting me through my entire journey in physics. From high school fascination, to undergrad at UT Austin, and then through the graduate program at Columbia, I want to thank you for listening and sharing in all the important memories we made together.

I am indebted to Kazuhiro Terao for taking me under your wing as a Columbia Postdoc. I am thankful for everything you have taught me especially how to program, give talks, think clearly, and to especially how to focus on the big picture.

I want to thank everyone in the Nevis group I have had the pleasure of working with especially David Kaleko, David Caratelli, Georgia Karagiorgi, Jose Crespo Anadon, Mark Ross-Lonergan, Davio Cianci, Kathryn Sutton, Yeon-Jae Jwa, and Iris Ponce. Thank you for making Nevis, Fermilab, and Columbia a enjoyable and productive place to work.

To my friends at Fermilab, especially those who were a part of our low energy excess working group, Jarett Moon, Chris Barnes, Rui An, Lauren Yates, Adrien Hourlier, and Taritree Wongjirad. I'll never forget the interesting time we had working on this analysis.

I finally want to thank my parents, Denise and Jim, and my brother, Sam, for supporting me along the path to my Ph.D. I am grateful for all the experiences we have shared and cannot thank ya'll enough for being a part of my life. 


\section{Chapter 1}

\section{Introduction}

This thesis describes the first automated search for the electron like low energy excess using image based reconstruction techniques in MicroBooNE. The MicroBooNE experiment is a large Liquid Argon Time Project Chamber (LArTPC) designed for precision measurements of short baseline accelerator neutrinos based at Fermilab National Accelerator Laboratory (Fermilab). LArTPCs are capable of producing high resolution images of particle interactions with excellent particle identification and energy reconstruction ability. A fully automated reconstruction chain based on computer vision techniques is developed and applied to MicroBooNE data represented as 2D images. The reconstruction chain combines traditional and deep learning techniques to probe the MiniBooNE electron-like low energy excess. Deep learning techniques for image classification, detection, and semantic segmentation have been successfully applied to LArTPC images for neutrino feature extraction and are the subject of two papers for which the author was a primary contributor [7] [8]. The excess is explored through a charge current quasi-elastic (CCQE) like topology featuring one lepton and one proton in the final state. The reconstruction and analysis chain is benchmarked on the Run 1 MicroBooNE data set equivalent to $5 \times 10^{19}$ protons on target (POT) from MicroBooNE's first period of data taking and represents about $4 \%$ of expected first result data set of $13.2 \times 10^{20}$ POT. The sensitivity to a low energy excess signal is extrapolated to the full $13.2 \times 10^{20}$ POT MicroBooNE dataset and explored in the context of a $3+1$ sterile neutrino model. 


\subsection{Overview of Issues Associated with Data Reconstruction in MicroBooNE}

This work presents an automated data reconstruction scheme for electron and muon type neutrino events in a large liquid argon detector called MicroBooNE. MicroBooNE was commissioned and began data taking in late 2015 and is on track to collect $13.2 \times 10^{20}$ protons on target by the end of Summer 2019. The analysis scheme described in this thesis is a new and independent approach to LArTPC data reconstruction based on computer vision and is an alternative approach to the traditional Pandora-based method [9]. This reconstruction paradigm considers individual charge deposition in wire and time space as atomic units for pattern recognition providing absolute granularity for neutrino identification. Data are represented in the form of $2 \mathrm{D}$ dimensional images and a suite of algorithms are described. Reconstruction and analysis of MicroBooNE data is challenging due to detector effects, the cosmogenic background rate, and the nature of low energy neutrino interactions. An event display of a MicroBooNE data event is shown in Fig. 1.1 and features an automatically reconstructed candidate CCQE interaction along with typical image features coming from cosmic ray and detector effects.

First, due to the millisecond drift time of the time projection chamber (TPC) readout coupled with MicroBooNE's location on the Earth's surface, an appreciable rate of cosmic ray muons occur in time with the neutrino interaction. In each beam triggered event tens or more cosmic ray particles traverse the detector producing both track and electromagnetic shower characteristics which can occlude neutrino interactions. The presence of cosmic ray tracks challenges automatic algorithms for reconstructing the neutrino features among an array of cosmic rays. Additionally, cosmic ray muons can fake electron neutrino interactions by decaying to a Michel electron.

Second, detector effects associated with the TPC and optical (PMT) systems can interfere with and introduce noise into the event image [10][11]. An important issue facing TPC based reconstruction algorithms is the presence of inactive or mis-configured wires on the three TPC planes. Wires which are inactive do not collect ionization signal and are interspersed throughout the three planes and cover approximately $10 \%$ of the TPC. Wire 
noise associated with the TPC electronics and wires themselves are a source detector noise which manifests in the event as spurious charge deposition. Due its proximity to the TPC wire planes, the PMT systems is also capable or producing spurious ionization signal. Algorithms for automatic pattern recognition of neutrino events rely heavily on simulated data for development and parameter optimization, therefore an accurate simulation is needed to understand the precise impact on the analysis.

A subset of the challenges related to electron neutrino reconstruction in MicroBooNE are shown in Fig. 1.1 .

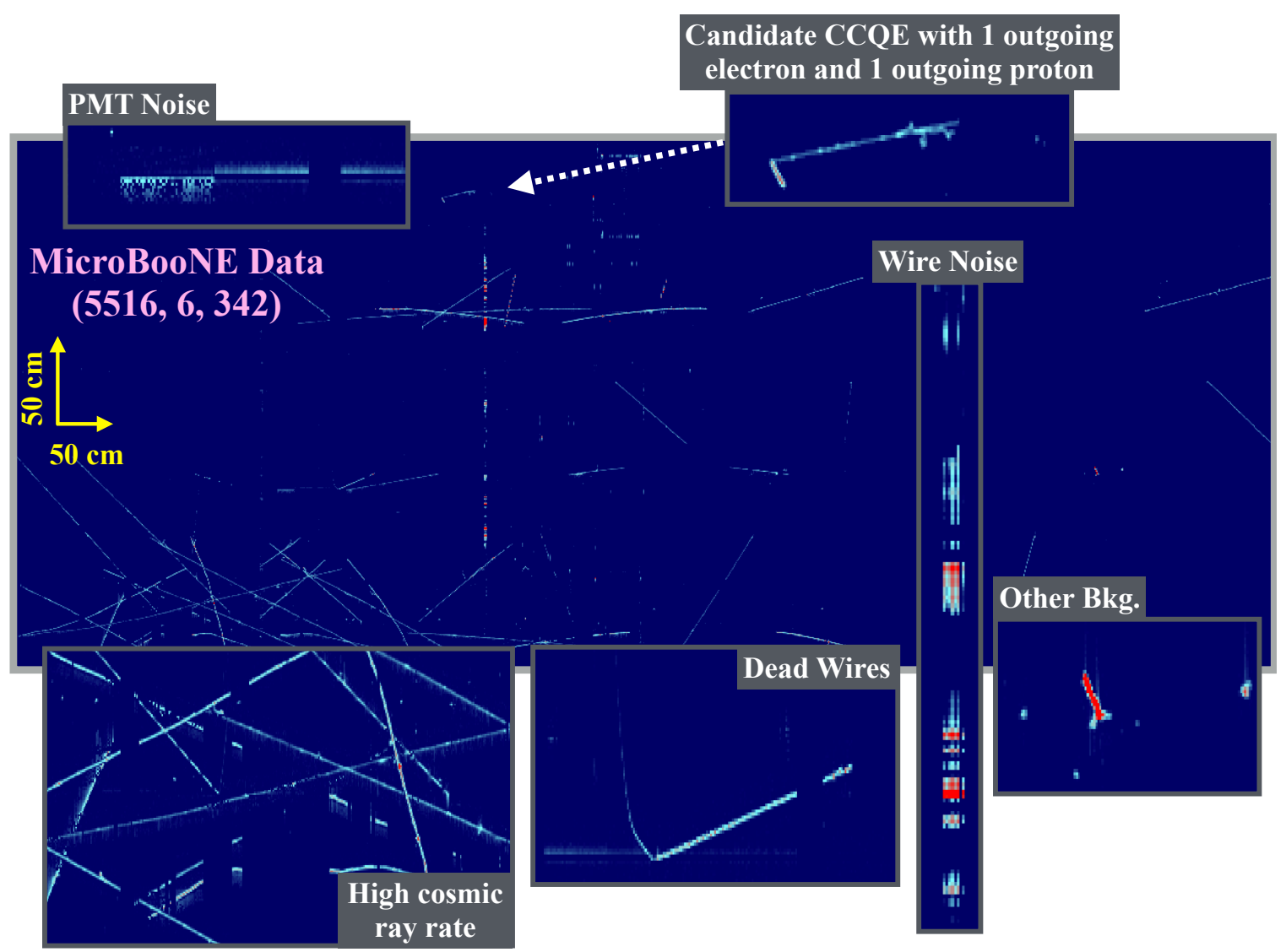

Figure 1.1: MicroBooNE data event display featuring a candidate CCQE interaction with one lepton and one proton in the final state. Examples of cosmogenic backgrounds and detector effects such as TPC and PMT noise and the presence of dead wires complicate automated reconstruction of neutrino interactions. 
The reconstruction strategy employed by this thesis targets a specific charge current quasi-elastic (CCQE) topology and does not provide generic pattern reconstruction for all types of neutrino scattering topologies as in the Pandora based approach. Accurate identification of hundreds of $\mathrm{MeV}$ electron neutrino interactions, as shown in Fig. 1.1, is challenged due to the spatial scale of the interaction with respect to other backgrounds. In addition, the topological features of the daughter particles must be extracted with detail for accurate particle identification. This image based reconstruction scheme locates the 3D scattering point of CCQE-like neutrinos, then extracts features of the final state particles for neutrino flavor and momentum estimation. Stringent cuts are placed on the daughter particle features to reduce the false positive rate of reconstructing background events for low energy excess and $3+1$ sterile oscillations searches.

\subsection{Outline of Thesis}

The work in this thesis is as follows. Chapter 2 provides an overview of neutrino interactions and neutrino oscillations including a discussion of oscillations in the context of 1 additional sterile neutrino. Next, Chapter 3 describes the MicroBooNE detector and the operating principle of the TPC, and the PMT optical systems. The Booster Neutrino Beam is detailed in Chapter 4 as the source of on-axis accelerator neutrinos for MicroBooNE. In Chapter 5 and Chapter 6 the low energy excess is discussed in the context of the LSND, MiniBooNE, and MicroBooNE short baseline experimental experiments. A review of the low energy excess observations in LSND and MiniBooNE experiments is presented. Next, the MiniBooNE $\nu_{e}$ CCQE data is unfolded to estimate the low energy excess spectra at MicroBooNE. Chapter 7 describes the final state topology selected to probe the low energy excess signal in MicroBooNE. Additionally, the event rate expectation for the low energy excess is characterized. Next, Chapter 8 provides an exhaustive presentation of the image based reconstruction software developed to reconstruct muon and electron neutrino interactions in MicroBooNE data. For readability, the author suggests reading the dedicated summary section provided in Section 8.2 and refer to the chapter contents if interested in the details. Muon and electron neutrino interactions are isolated using 2D and 3D features 
derived from image based reconstruction in Chapter 9 and compared to the Run 1 MicroBooNE data. A discussion of systematic errors in the context of the low energy excess search along with the $\nu_{\mu}$ constraint is presented in Chapter 10. MicroBooNE's ability to distinguish a MiniBooNE like low energy excess signal is described in Chapter 11 along with the experimental sensitivity to $3+1$ sterile neutrino oscillations. Finally, a summary of the results from the thesis are summarized in the concluding chapter, Chapter 12 . 


\section{Chapter 2}

\section{Neutrino Physics and Oscillations}

This chapter reviews the physics of neutrino particles and the role they play in the Standard Model of physics. A survey of neutrino physics is described along with a detailed discussion of neutrino oscillations. Finally, an overview of $3+1$ sterile neutrino oscillations is presented.

\subsection{Introduction to Neutrinos}

The standard model of electro-weak interactions includes three active neutrino flavors accompanying the charged leptons electron, muon, and tau. Neutrinos are part of left handed doublets under $\mathrm{SU}(2)$,

$$
\nu_{L l}=\left(\begin{array}{c}
\nu_{l} \\
l
\end{array}\right)_{L},
$$

where $l=e, \mu, \tau$. Two gauge bosons are predicted by the electro-weak theory, $W^{ \pm}$and $Z^{0}$, as well as $\mathrm{SU}(2)$ and $\mathrm{U}(1)$ gauge coupling constants, $g$ and $g^{\prime}$. The charged leptons and quarks are coupled to neutrinos through charge current interactions mediated by the $W$ boson given by the Lagrangian,

$$
\mathcal{L}_{\mathrm{CC}}=-\frac{g}{2 \sqrt{2}} \sum_{l} W_{\mu}^{+} \bar{\nu}_{L l} \gamma^{\mu}\left(1-\gamma^{5}\right) l_{L}^{-}+\text {h.c. }
$$

Neutrinos can also interact weakly via neutral current interactions mediated by the $Z^{0}$

boson. At present the measurement of the $Z^{0}$ boson decay constrains the active number of left handed neutrinos to $2.991 \pm 0.007$ [6]. In the Standard Model neutrinos have zero mass 
and carry a lepton quantum number. First postulated in 1930 by Pauli, and later observed by Reines and Cowan in 1956, the neutrino has been probed extensively by a variety of experiments around the globe. With the advent of clear evidence for neutrino oscillations in 2001 by the SNO collaboration, theoretical and experimental activity in neutrino physics remains strong into the $21^{\text {st }}$ century.

\subsection{Neutrino Oscillations}

Observational evidence of neutrino oscillations requires that the active flavor states, charged under the weak interaction, are not the same as neutrino mass eigenstates. The eigenstates are related by a unitary mixing matrix,

$$
\nu_{\alpha}=\sum U_{\alpha i} \nu_{i}
$$

where $\alpha=e, \mu, \tau$ are called the flavor eigenstates, and $i=1,2,3$ are called the mass eigenstates. The matrix $U$ is called the Pontecorvo-Maki-Nakagawa-Sakata (PMNS) matrix. Neutrino mass eigenstates are eigenstates of the free-particle Hamiltonian,

$$
\left|\nu_{i}\right\rangle=e^{-i p_{i} x}\left|\nu_{i}\right\rangle
$$

where $E_{i}^{2}=m_{i}^{2}+|\vec{p}|^{2}$. Consider two neutrino mixing with an angle $\theta$,

$$
U=\left(\begin{array}{cc}
\cos \theta & \sin \theta \\
-\sin \theta & \cos \theta
\end{array}\right) .
$$

Then the flavor eigenstates $\left|\nu_{\alpha}\right\rangle$ and $\left|\nu_{\beta}\right\rangle$ can be expressed as a function of position and time as,

$$
\begin{aligned}
& \left|\nu_{\alpha}(t, \vec{x})\right\rangle=\cos \theta e^{-i p_{1} t}\left|\nu_{1}\right\rangle+\sin \theta e^{-i p_{2} t}\left|\nu_{2}\right\rangle \\
& \left|\nu_{\beta}(t, \vec{x})\right\rangle=-\sin \theta e^{-i p_{1} t}\left|\nu_{1}\right\rangle+\cos \theta e^{-i p_{2} t}\left|\nu_{2}\right\rangle .
\end{aligned}
$$

In the ultra-relativistic limit, $t \sim L$ the distance travelled by the neutrino and $E_{i}-p_{z, i} \approx$ $m_{i}^{2} / 2 E_{i}$. Then the probability for $\left|\nu_{\alpha}\right\rangle$ to $\left|\nu_{\beta}\right\rangle$ transitions is given by,

$$
\begin{aligned}
P_{\alpha \rightarrow \beta}(L) & =\left|\left\langle\nu_{\beta} \mid \nu_{\alpha}(t, z=L)\right\rangle\right|^{2} \\
& =\sin ^{2} 2 \theta \sin ^{2}\left(1.27 \frac{\Delta m^{2} L}{E_{\nu}}\right) .
\end{aligned}
$$


In this equation $E$ is the neutrino energy, $L$ is the distance from the source, and $\Delta m^{2}=$ $\left|m_{2}^{2}-m_{1}^{2}\right|$ is the magnitude difference between the $\left|\nu_{1}\right\rangle$ and $\left|\nu_{2}\right\rangle$ mass eigenstates. The result predicts that a pure flavor eigenstate created via weak decay can oscillate into other flavors by spatial propagation. Three neutrino mixing is an extension of two neutrino mixing and is specified by three rotation angles $\theta_{23}, \theta_{13}$, and $\theta_{12}\left(0 \leq \theta_{i} \leq \pi / 2\right)$ and three CP-violating phases $\delta, \phi_{2}$, and $\phi_{3}$. The three neutrino mixing matrix can be written as,

$$
U=\left[\begin{array}{ccc}
1 & 0 & 0 \\
0 & c_{23} & s_{23} \\
0 & -s_{23} & c_{23}
\end{array}\right]\left[\begin{array}{ccc}
c_{13} & 0 & s_{13} e^{-i \delta} \\
0 & 1 & 0 \\
-s_{13} e^{i \delta} & 0 & c_{13}
\end{array}\right]\left[\begin{array}{ccc}
c_{12} & s_{12} & 0 \\
-s_{12} & c_{12} & 0 \\
0 & 0 & 1
\end{array}\right]\left[\begin{array}{ccc}
1 & 0 & 0 \\
0 & e^{i \phi_{2} / 2} & 0 \\
0 & 0 & e^{i \phi_{3} / 2}
\end{array}\right]
$$

here $c_{j k}$ denotes $\cos \theta_{j k}$ and $s_{j k}$ denotes $\sin \theta_{j k}$. The angle $\theta_{23}$ is relevant for oscillations of atmospherics neutrinos, the angle $\theta_{12}$ describes solar neutrino oscillations, and the angle $\theta_{13}$ is measured by reactor neutrino experiments at short baselines. The two Majorana phases $\phi_{2}$ and $\phi_{3}$ do not effect the oscillations probabilities. An ambiguity in mass ordering of the neutrino mass eigenstates arises from the unknown sign of $\Delta m_{23}^{2}$. If $\nu_{1}$ happens to be the lightest neutrino eigenstate, the ordering is called "normal" $\left(m_{1}<m_{2}<m_{3}\right)$, otherwise is labelled as "inverted" $\left(m_{3}<m_{1}<m_{2}\right)$. An diagram of the mass splitting is shown in Fig. 2.1.

Neutrino experiments are designed to search for neutrino oscillations via neutrino appearance or disappearance. In appearance experiments the neutrinos of known flavor $\alpha$ are produced and measured a distance away from the source as another flavor $\beta$. In disappearance searches neutrinos of a given flavor are produced and the same neutrino flavor is measured. A given experiment optimizes the distance between the source and detector, $L$, with the given neutrino energy, $E$, for maximal sensitivity to a given type of oscillations. A table of the current oscillation parameters [6] is given in Table 2.1 for the normal hierarchy.

\subsection{Sterile Neutrinos}

Recent experiments performed at short baseline facilities, LSND and MiniBooNE, have observed apparent excess of electron type neutrinos in primarily muon neutrino beams. These experiments, described in Chapter 5, have measured signals consistent with two 


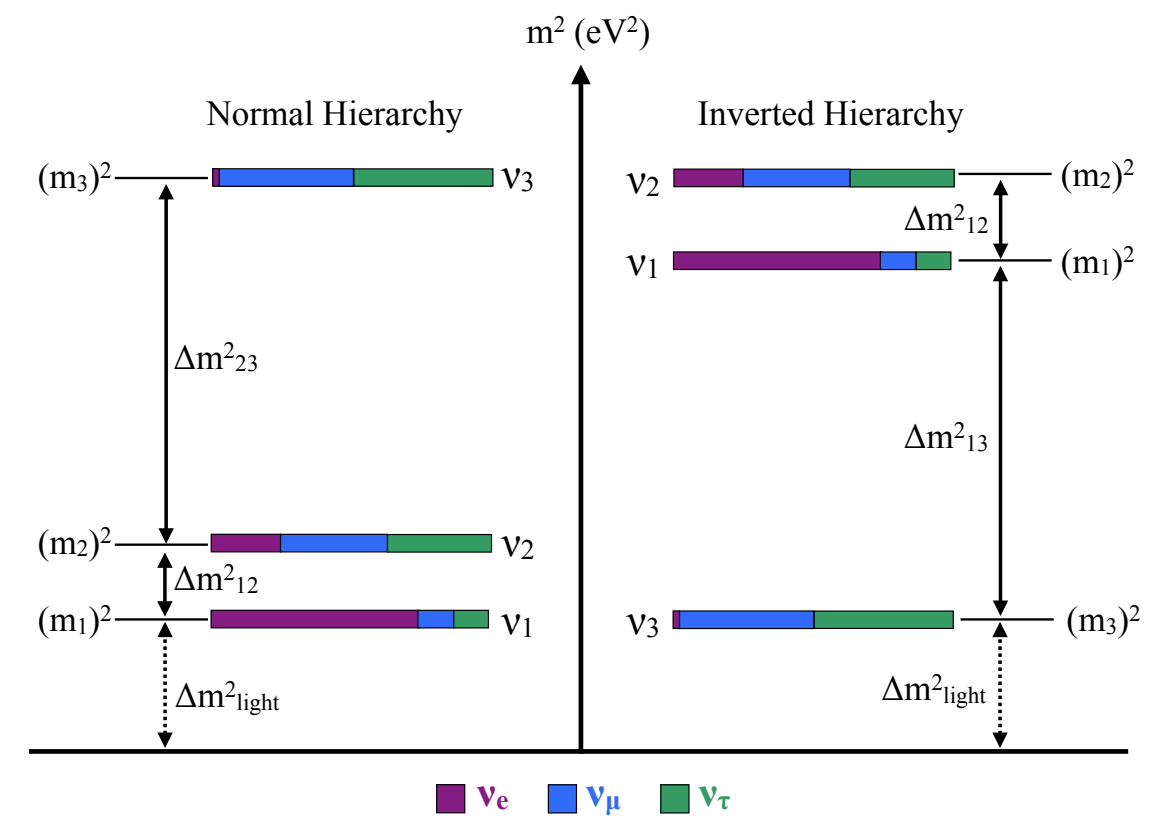

Figure 2.1: Three neutrino mass spectrum for normal (left) and inverted (right) hierarchy. The lepton content of each mass eigenstate is denoted in color and represents the level of mixing with each weak eigenstate.

\begin{tabular}{c|r} 
Parameter & Value (Normal Order) \\
\hline $\sin ^{2} \theta_{12}$ & $0.307 \pm 0.014$ \\
$\sin ^{2} \theta_{23}$ & $0.417 \pm 0.028$ \\
$\sin ^{2} \theta_{13}$ & $(2.12 \pm 0.08) \times 10^{-2}$ \\
$\Delta m_{21}^{2}$ & $(7.53 \pm 0.18) \times 10^{-5} \mathrm{eV}^{2}$ \\
$\Delta m_{32}^{2}$ & $(2.51 \pm 0.05) \times 10^{-3} \mathrm{eV}^{2}$
\end{tabular}

Table 2.1: Neutrino oscillation parameters as of 2018 provided by the Particle Data Group) [6].

neutrino oscillations at $\Delta m^{2} \geq 0.1 \mathrm{eV}^{2}$, inconsistent with the mass splitting for solar and atmospheric oscillations. Introducing the LSND and MiniBooNE signals into the three neutrino paradigm, such as via atmospheric mixing of high and low $\Delta m^{2}$ values, yields global fits which are inconsistent with data [12]. The simplest extension to the three neutrino 
model is the addition of a single sterile neutrino, $s$, which mixes with the three active flavors but is unable to interact weakly. The mixing matrix with one additional flavor state is,

$$
U_{3+1}=\left[\begin{array}{cccc}
U_{e 1} & U_{e 2} & U_{e 3} & U_{e 4} \\
\vdots & & \vdots & U_{\mu 4} \\
\vdots & & \vdots & U_{\tau 4} \\
U_{s 1} & U_{s 2} & U_{s 3} & U_{s 4}
\end{array}\right]
$$

The mass splitting with an additional sterile state is shown in Fig. 2.2 and is called the $3+1$ model.

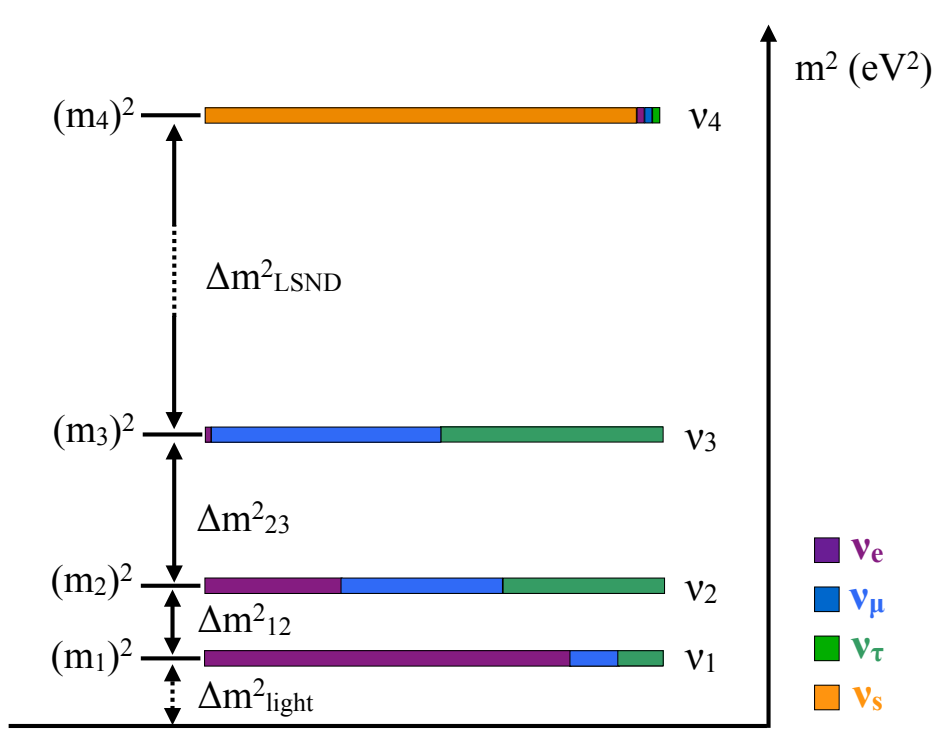

Figure 2.2: Illustration of three neutrino mass states and their relationship with the fourth sterile neutrino mass splitting.

Additional sterile neutrino states have important consequences outside of oscillation experiments. Precise measurements of the $\mathrm{Z}$ boson constrain the number of active neutrino flavors and hence an additional neutrino state must be "sterile" under the weak interaction. An additional sterile state has cosmological ramifications as well, which at the time of writing are not precise enough to make a statement. While sterile neutrinos may not interact weakly, they could interact through a new force of nature weaker than the weakinteraction scale making cosmological measurements insensitive to a sterile signal. Finally, 
the sterile neutrino state will distort the beta decay spectrum and new experiments are coming online to address this. This thesis explores the appearance of electron neutrinos in a primarily muon neutrino beam at a the Fermilab short baseline facility. 


\section{Chapter 3}

\section{The MicroBooNE Detector}

This chapter describes the MicroBooNE detector located at Fermilab National Accelerator Laboratory. MicroBooNE is the first large scale liquid argon time projection chamber (LArTPC) exposed to a high intensity neutrino beam in the United States. LArTPCs combine high spatial resolution with total calorimetry for particle identification using liquid argon as a detection medium. The MicroBooNE detector consists of a time projection chamber (TPC), a PMT optical system, and an external cosmic ray tagging device. The TPC system features three wire planes readout of ionization of charged particles traversing the detector enabling 3D position reconstruction. The PMT system collects scintillation light produced by argon and is used for beam triggering and particle reconstruction. The external cosmic ray tagging system features an array of scintillator panels surrounding the detector and is not described here. MicroBooNE began operations in late 2015 and has since been taking neutrino mode beam data to address short baseline neutrino oscillations and test the nature of the MiniBooNE low energy excess.

\subsection{Introduction}

The Micro-Booster Neutrino Experiment (MicroBooNE) is a liquid argon time projection chamber (LArTPC) with an active mass of 90 tons located in Batavia, Illinois at the Fermilab National Accelerator Laboratory (FNAL). The detector is located at the Liquid Argon Test Facility (LArTF) on axis to the Booster Neutrino Beam (BNB), $470 \mathrm{~m}$ downstream 
from the proton target. An arial diagram of the experimental site is shown in Fig. 3.1

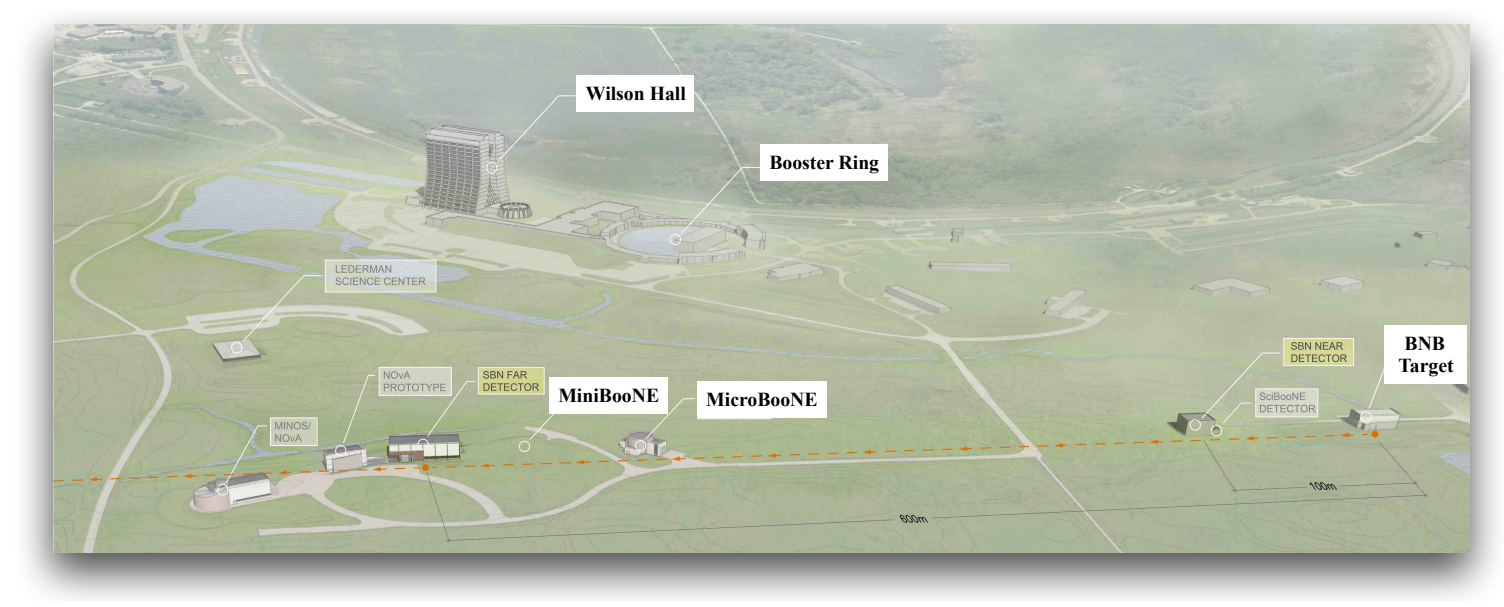

Figure 3.1: Overview of the Fermilab experimental site showing the location of MicroBooNE along the BNB beam line.

The detector operates within a cryogenic vessel which houses the TPC and optical systems. A detector schematic is shown in Fig. 3.2. The operating principle of a LArTPC detector involves a homogenous volume of liquid argon exposed to an external electric field. Particles traversing the detector volume produce scintillation light and ionize argon atoms leaving a trail of ionization electrons. The electrons drift in the electric field to a set of electrically charged sense wires where the ionization electrons are recorded as a function of time. The result is a high resolution image of charged particle trajectories.

\subsection{Time Projection Chamber}

The MicroBooNE Time Projection Chamber (TPC) drifts and collects ionization electrons produced by charged particles traversing the detector. The TPC is composed of three components: the cathode, anode, and field cage and are shown in Fig. 3.3.

The cathode plane is a stainless steel sheet held at an electric potential of $-70 \mathrm{kV}$. A uniform $273 \mathrm{~V} / \mathrm{cm}$ electric field between the cathode and anode planes is established inside the field cage. The field cage consists of stainless steel tubes connected via a resistor chain which steps down the voltage toward the anode. The anode plane houses three planes of gold-plated copper sense-wires, separated by $3 \mathrm{~mm}$, and oriented at 60 degrees with 


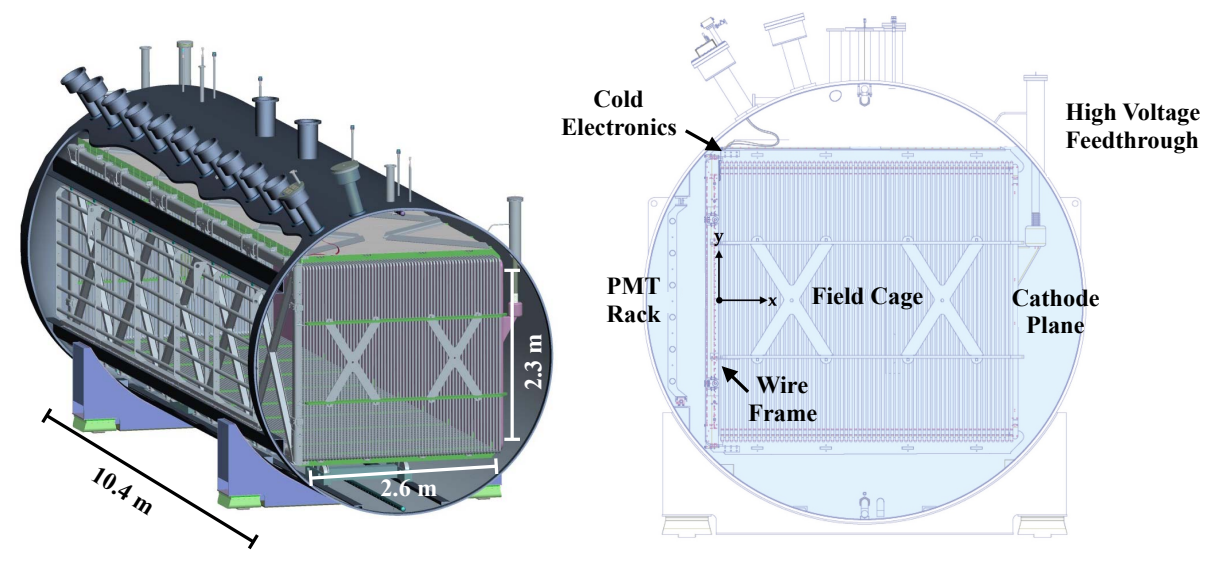

Figure 3.2: Left: Schematic of the MicroBooNE cryostat (outer cylinder). The top of the detector features feed through connections for interfacing warm and cold electronics. On side of the field cage can be seen within the cryostat. Right: Cross section of the MicroBooNE detector with notable components labelled.
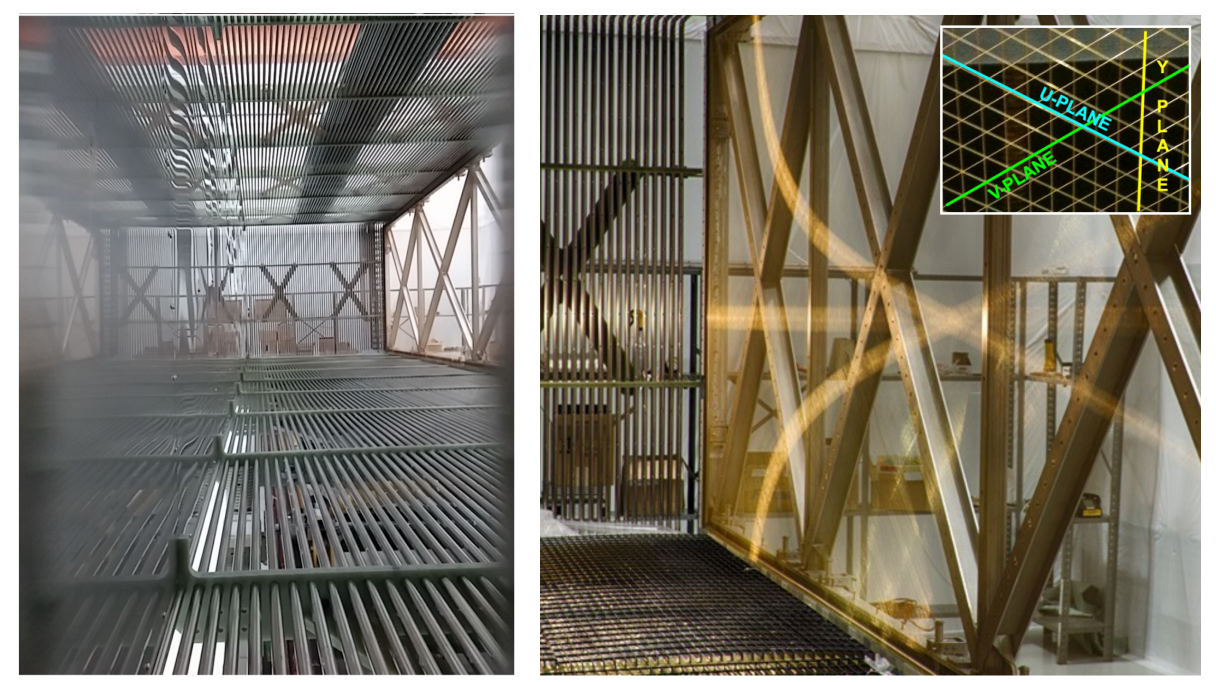

Figure 3.3: Left: Image shows the cathode plane surrounded by the stainless steel tubes which comprise the field cage. Right: The three anode plane sense wires are shown. Insert shows the orientation of wires of the $\mathrm{U}, \mathrm{V}$, and $\mathrm{Y}$ planes.

respect to one another. The "U" and "V" sense-wire planes are called induction planes as ionization electrons pass nearby and produce signal via induction. The induction planes are oriented at \pm 60 degrees with respect to the vertical and consist of 2400 wires each. The 
ionization charge is collected on the "Y" wire plane, called the collection plane, which is oriented vertically and consists of 3456 wires. Each wire plane is held at a bias voltage to achieve transparency of the first two induction wire planes. By collecting 2D projections of ionization signal on two or more wire planes, the $3 \mathrm{D}$ configuration of the event can be recovered. A schematic of the TPC operating principle is shown in Fig. 3.4

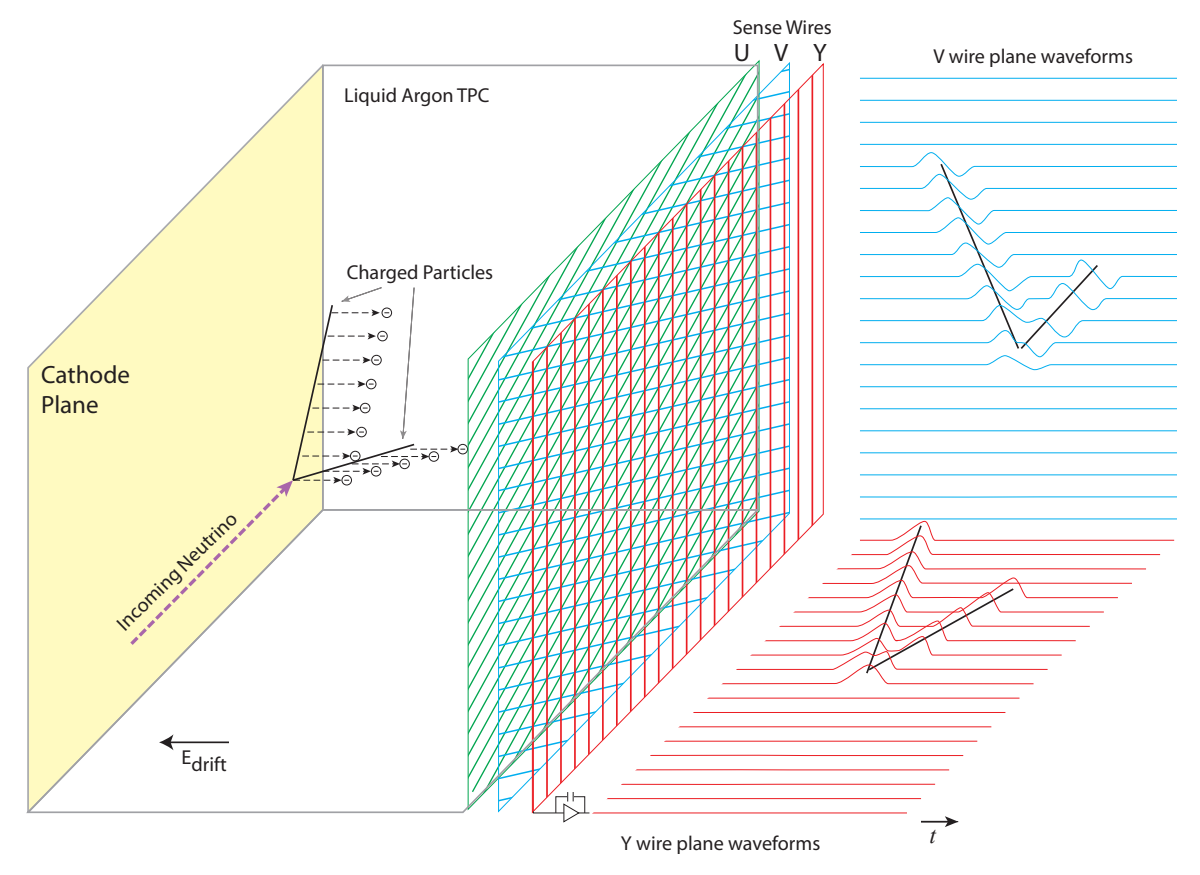

Figure 3.4: LArTPC signal formation diagram described in Section 3.2 .

In Fig. 3.4 an incoming neutrino particle scatters on an argon nucleus producing two forward going charged particles. The charged particles ionize the argon producing a cloud of ionization electrons. The electron cloud is detected on the three planes with wires separated by $3 \mathrm{~mm}$. The signal on the $\mathrm{V}$ induction plane is shown blue and is bipolar in nature. The electron cloud is deposited on the $\mathrm{Y}$ collection plane shown in red. The two track pattern can be observed as a function of drift time, $t$, and spatial extent of the wires. The spatial location of a particle's trajectory inside the detector volume can be reconstructed by determining the $\mathrm{X}, \mathrm{Y}$, and $\mathrm{Z}$ location of the electron cloud. The $\mathrm{X}$ position coordinate can be determined by multipling the electron drift speed by the drift time coordinate. The $\mathrm{Y}$ and $\mathrm{Z}$ position is determined by the wire overlap between planes, and wire number respectively. An example 
neutrino event observed in the MicroBooNE detector as projected on the three wire planes is shown in Fig. 3.5 .

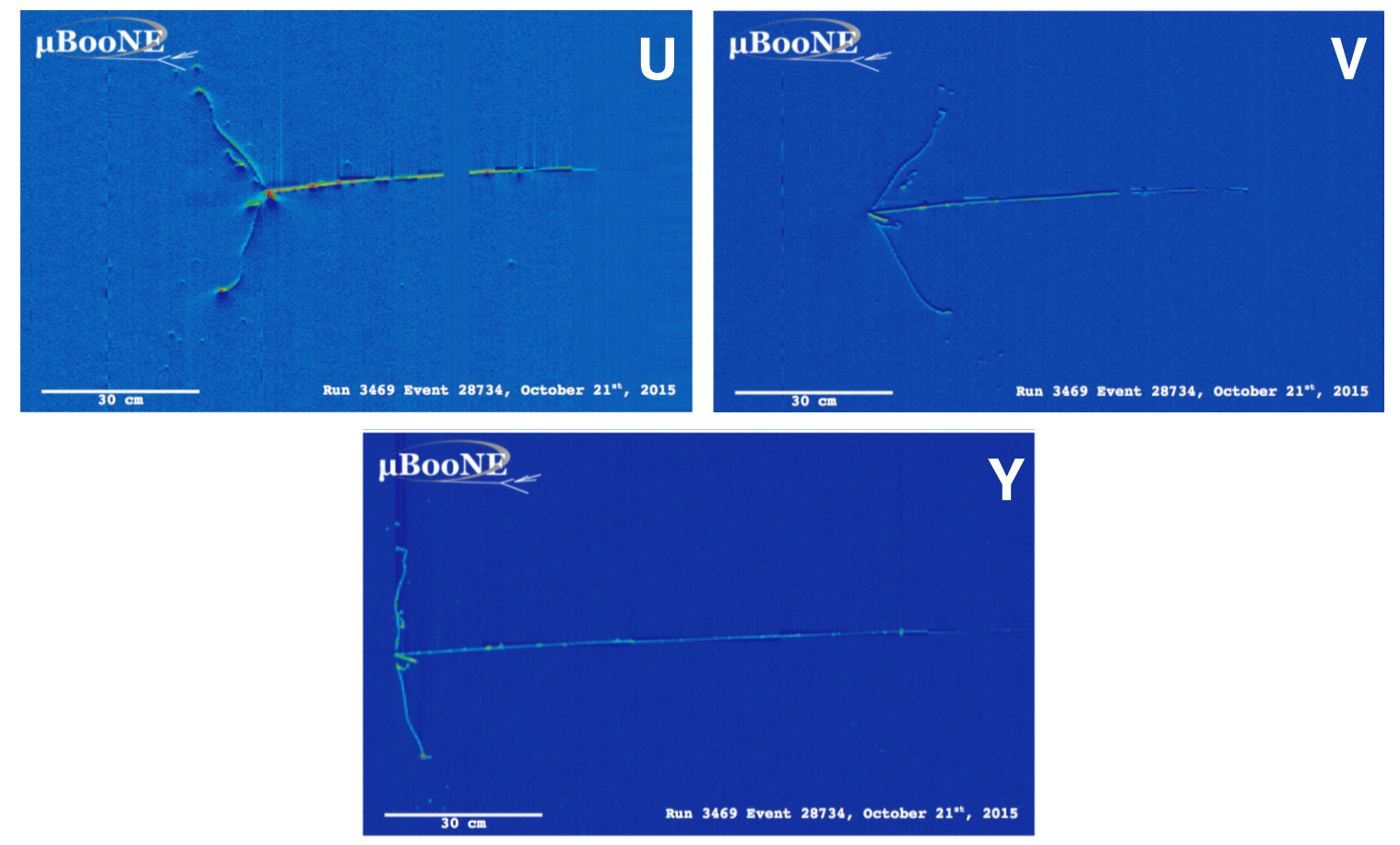

Figure 3.5: Example neutrino candidate event observed in MicroBooNE. The same interaction is shown on the $\mathrm{U}, \mathrm{V}$, and $\mathrm{Y}$ wire planes. A candidate muon, proton, and two photons from $\pi^{0}$ decay are likely present as final state particles.

\subsection{Optical System}

The MicroBooNE optical system collects scintillation light produced by excited argon atoms and is an important input for triggering on neutrino interactions. Neutrino interactions from the beam produce a large amount of light which are detected by an array of 32 photomultipliers located behind the wire planes and facing the cathode. Wire signals alone are not sufficient to determine the absolute drift location of particles as an ambiguity is introduced due to the millisecond length of the TPC readout time. Scintillation light which arrives on the order of nanoseconds can accurately determine the event timing. Scintillation light is produced in liquid argon by the formation and subsequent radiative decay of excited 
argon dimers which yield tens of thousands of photons per MeV of energy. Scintillation light is emitted isotropically with a wavelength of $128 \mathrm{~nm}$ and arrives on nanosecond time scales to the PMT array without re-absorption by argon. Each optical unit consists of 8-inch cryogenic Hamamatsu PMTs covered with a flat acrylic plate. The acrylic plate is coated with a mixture of Tetraphenyl Butadiene (TPB) and polystyrene. TPB is an organic molecule which absorbs UV light and re-emits in the visual range at $\sim 425 \mathrm{~nm}$. Photons are re-emitted isotropically by the plate which leads to an inefficiency for light collection. A diagram of a MicroBooNE PMT and layout of the cathode facing PMT rack is shown in Fig. 3.6.
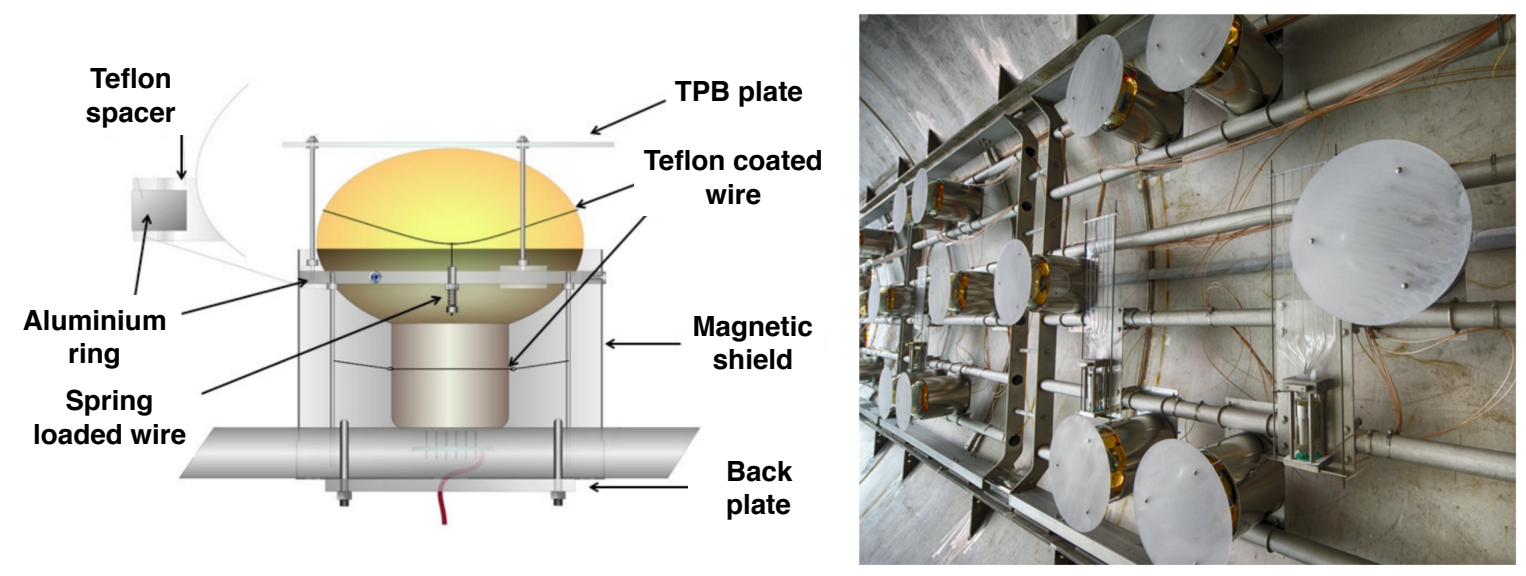

Figure 3.6: Left: Diagram of a MicroBooNE 8-inch PMT. Right: PMT rack array located behind the anode wire planes and mounted on the MicroBooNE cryostat wall.

\subsection{Electronics Readout and Event Trigger}

The TPC and optical systems form analog signals in the cold which are read out and digitized in warm electronics crates located above the cryostat. The cold electronics feature CMOS ASIC devices mounted on a dedicated motherboard for signal amplification and shaping. The ASIC applies a gain of $14 \mathrm{mV} / \mathrm{fC}$ and a $2 \mu$ s shaping time. Cold cables transmit signals through feedthroughs mounted on the cryostat to warm electronics crates where the signals are digitized and formatted before being shipped to the data acquisition system. The front end modules (FEMs) TPC signals are digitized at $16 \mathrm{MHz}$ sampling 
with 12 bits resolution, then processed by an FPGA chip where they are down sampled to $2 \mathrm{MHz}$. The collection of three wire planes is read out in three $1.6 \mathrm{~ms}$ frames. The frame size is chosen based on the time for ionization electrons to travel in the electric field from the far end of the detector. One frame is recorded prior to the trigger, and two after. The data rate is reduced by applying a Huffman coding scheme.

PMT signals are processed in a similar fashion. PMT signals are first shaped into a positive unipolar shape with a $60 \mathrm{~ns}$ shaping time which returns to baseline at approximately $200 \mathrm{~ns}$. The signal is then digitized at $64 \mathrm{MHz}$ sampling. There are two types of PMT readout, beam and cosmic discriminator read out. The beam discriminator has no threshold and reads out 1500 samples $(23.4 \mu \mathrm{s})$ around the beam spill. The PMTs dynamic range is captured by reading out a high gain and low gain channel simultaneously. TPC and PMT data are finally transmitted via optical links from a transmit board (XMIT) located in each crate to PCI express ports on data acquisition PCs (DAQ). A schematic of the detector readout is shown in Fig. 3.7

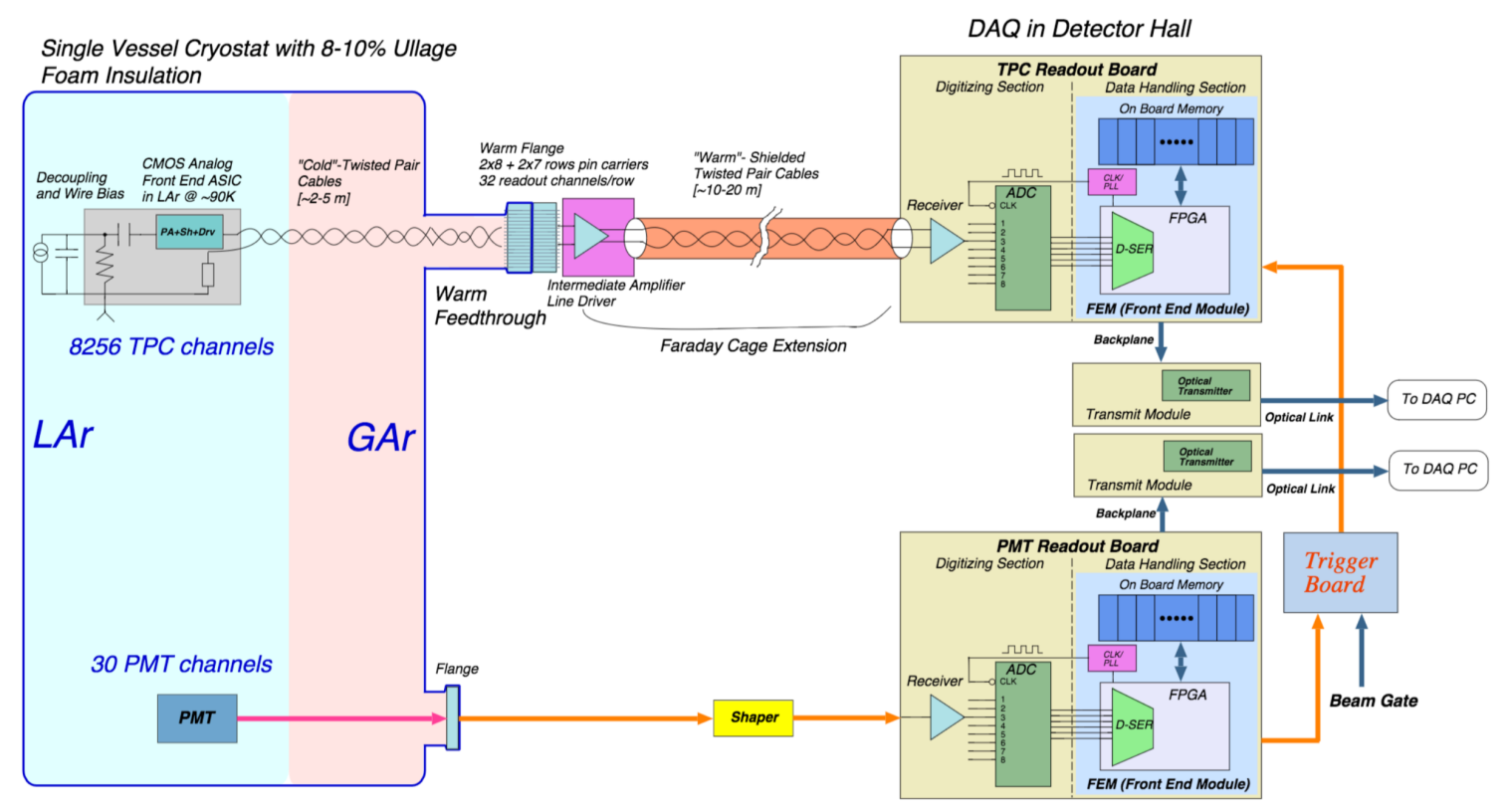

Figure 3.7: MicroBooNE readout schematic. The cold electronics located within the detector are shown in the left including the TPC and PMT channels. Data are read out via cables through warm feedthroughs and the flange to racks of electronics located on the right. A trigger board hosts accelerator signals, and external triggering logic for detector readout. 
A continuous TPC stream of data is also in operation at MicroBooNE which enables the detection of supernova neutrinos, called the "supernova stream". Supernova stream signals are lossy compressed in the FPGA by applying a channel-wise ADC threshold. This stream continually reads out TPC signals into a temporary storage buffer on the DAQ machines awaiting a Supernova Early Warning System (SNEWS) alert [13].

TPC and PMT signals are read out when a trigger is issued to controller boards located in the readout crates. MicroBooNE receives signals from the Fermilab accelerator complex when beam is expected, called the BNB trigger. This trigger causes a beam gate window to open in the PMT and TPC readout for data taking. As most BNB triggers do not result in a neutrino interaction, an additional software based trigger is employed to determine the presence of optical activity above threshold within the detector. A software based trigger allows for higher complexity algorithms to be deployed an a shorter timescale. The algorithms require 6.5 effective $\mathrm{PE}$ of optical activity in the $1.6 \mu \mathrm{s}$ beam spill window and results in a rejection of $\sim 97 \%$ of spills. Optical activity within the BNB beam-gate window is shown in Fig. 3.8 ,

Triggers unassociated with the beam are called external triggers and are used to collect background data such as cosmic ray only interactions. External BNB events, or EXT-BNB, are manual triggers of the detector which pass the software trigger requirements and represent events where no neutrino interaction is present, but the optical activity indicates detector activity. EXT-BNB events are extensively used in this thesis for cosmic ray background studies. Once the readout is complete the data are shipped to the DAQ machines for packaging into a specialty file format and then stored on tape. 


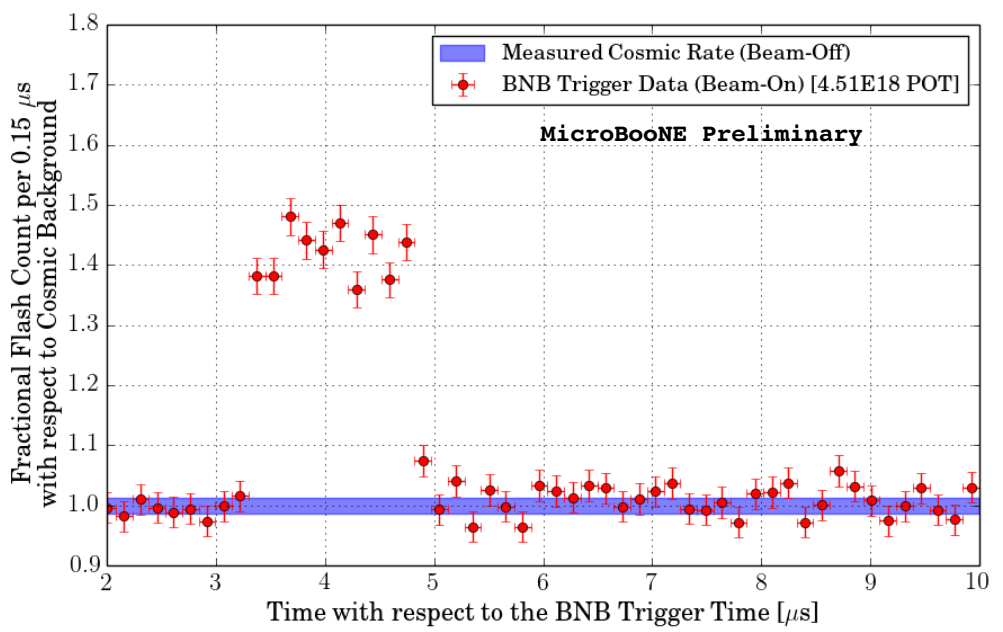

Figure 3.8: The measured distribution of flash times (requiring flashes greater than $50 \mathrm{PE}$ ) with respect to the trigger time for BNB-triggered events, shown as a ratio to the expected cosmic rate from off-beam data [1]. The blue band denoting the cosmic rate was centered at one, with a width corresponding to the measured uncertainty in the cosmic rate. A clear excess can be seen due to neutrinos between 3 and $5 \mu$ s after the trigger where neutrinos are expected. 


\section{Chapter 4}

\section{The Booster Neutrino Beam}

The purpose of this chapter is to describe the Booster Neutrino Beam-line (BNB) located at Fermilab. The beamline uses $8 \mathrm{GeV}$ protons from the BNB directed onto a beryllium target. The secondary hadrons are focused by a magnetic horn down a long decay pipe. Decays of secondaries then give rise to a neutrino beam which is directed at the MicroBooNE detector. The beam, proton target and focusing horn, and the beam composition are described. The flux of neutrinos at MicroBooNE is also described. It is important to understand the flux of muon and electron neutrinos at MicroBooNE to set the analysis goals for probing a low energy signal.

\subsection{The Booster Proton Beam}

Protons in the BNB originate from hydrogen gas molecules which are ionized to $\mathrm{H}^{-}$using a Cockcroft-Walton generator and then directed into a linear accelerator machine (LINAC). In the LINAC, the negatively charged ions are accelerated from $750 \mathrm{keV}$ to $400 \mathrm{MeV}$ in two stages, a low and high energy section, via a series of radio frequency (RF) cavities. The 400 $\mathrm{MeV}$ ions exiting the LINAC are injected into the booster facility by passing them through a stripping foil to remove electrons. The booster machine is a rapidly cycling synchrotron which accelerates protons from $400 \mathrm{MeV}$ to $8 \mathrm{GeV}$ kinetic energy at a rate of $15 \mathrm{~Hz}$ using a series of magnets and RF cavities. The protons are bunched into 81 units, each 2 ns wide, and spaced in $1.6 \mu \mathrm{s}$ batches. Each batch has a maximum allowable repetition rate for 
delivery of protons to the target of $5 \mathrm{~Hz}$ with approximately $5 \times 10^{12}$ protons per pulse. The number of protons directed on target (POT) is measured by two toroids upstream of the target as part of a larger beam monitoring system which includes beam position monitors, a resistive wall monitor, and multi-wire chamber which monitor beam characteristics. A schematic of the accelerator complex is shown in Fig. 4.1 .

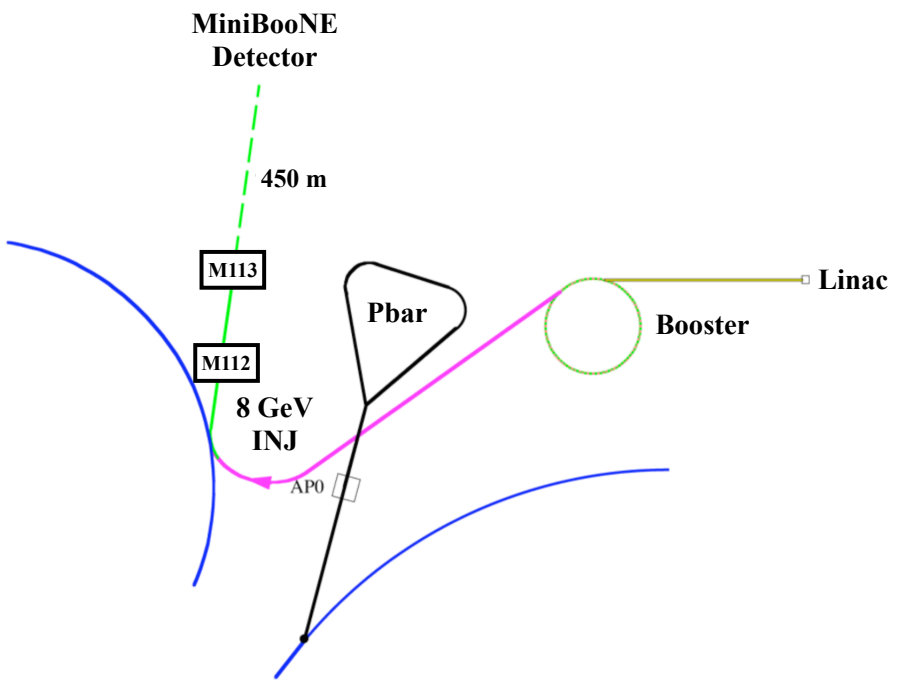

Figure 4.1: Overhead view of the Fermilab accelerator complex surrounding the BNB. The MicroBooNE detector is located on the same beam line as the MiniBooNE detector.

\subsection{Proton Target and Focusing Horn}

The $8 \mathrm{GeV}$ proton beam strikes a beryllium target of $71.1 \mathrm{~cm}$ in length and $0.51 \mathrm{~cm}$ in radius. Beryllium was chosen due to its thermal and mechanical properties, high pion yield, and low energy deposition per unit length, which minimizes the load on the cooling system. Additionally, beryllium was chosen to minimize the remnant radioactivity due to proton exposure. The target itself is located within a larger focusing magnet, called the horn, which is an aluminum alloy toroidal electromagnet. The target and a schematic of the horn are shown in Fig. 4.2 .

High energy protons interact inside the beryllium target and produce primarily charged pions with a significant fraction of charged kaons. The magnet focusing horn sign selects 

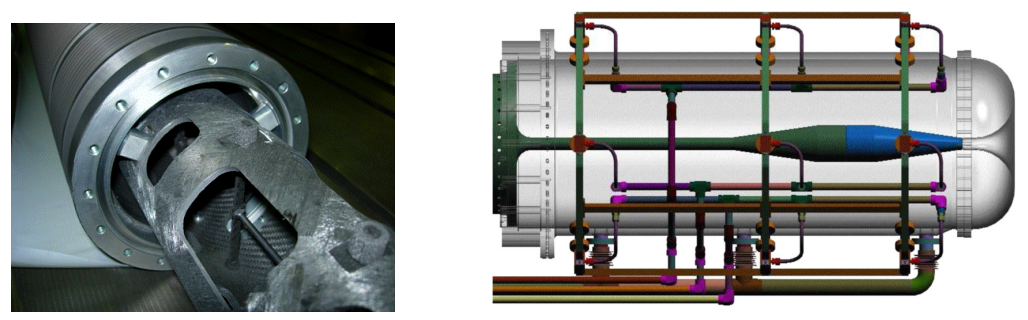

Figure 4.2: Left: BNB target enclosure. Right: Magnetic focusing horn schematic. The target rests inside within the inner cylindrical cavity (green).

postive or negatively charged mesons by tuning the direction of the magnetic field. Other secondary particles are deflected away from the decay region due to variations in their mass and energy. The normal horn configuration selects positively charged pions for neutrino production as shown in Fig. 4.3 .

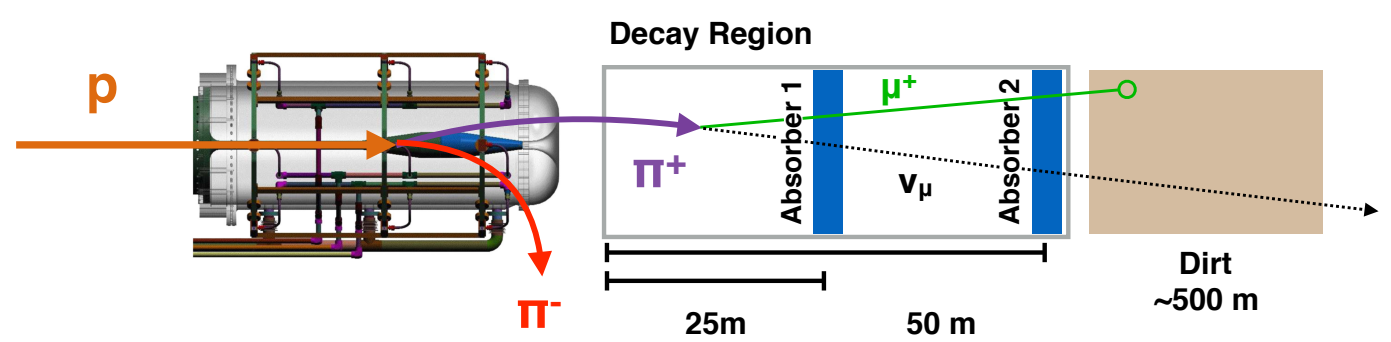

Figure 4.3: Diagram of an incident proton beam on the target located within the magnetic focusing horn. Running in the neutrino mode, the horn selects $\pi^{+}$particles for passage into a $50 \mathrm{~m}$ long decay region. The pions decay to a muon type particle and neutrino in the decay region. The muon type particle is absorbed in the dirt, while the neutrino travels onward in the beam direction.

The horn operates at up to a $5 \mathrm{~Hz}$ rate, with a $170 \mathrm{kA}$ pulse lasting for $150 \mu$ s producing a maximum magnetic field of $1.5 \mathrm{~T}$. The horn focuses pions into a 50 meter decay region which is a steel pipe of two meters in diameter. At the end of the decay pipe a steel absorber stops secondaries produced in the target except for neutrinos. An additional absorber located at $25 \mathrm{~m}$ can be lowered into the decay pipe to modulate the rate of secondaries. The neutrinos then travel a distance of $470 \mathrm{~m}$ to the MicroBooNE detector. 


\subsection{Beam Composition}

The MicroBooNE beam is largely dominated by $\nu_{\mu}$ which are produced via the $\pi^{+} \rightarrow \mu^{+} \nu_{\mu}$ decay chain with some contamination of $\nu_{e}$ from subsequent $\mu^{+}$decay via $\mu^{+} \rightarrow e^{+} \nu_{e} \bar{\nu}_{\mu}$. The $\pi^{+}$meson can instead decay directly to $e^{+}$and $\nu_{e}$ but is suppressed relative to muon neutrino production by a factor of $10^{-4}$. Kaon type particles contribute to the neutrino flux at energies $2.5 \mathrm{GeV}$ and above. The neutrino flux prediction at MicroBooNE uses the Booster Neutrino Beam Monte-Carlo developed by the MiniBooNE collaboration which includes a full GEANT4 simulation of the beam-line, focusing horn, and decay region. The full simulation details are described in [14]. The flux prediction for the MicroBooNE detector reuses the same simulation with modified detector parameters. The primary uncertainty on the $\pi^{+}$production is estimated by propagating errors via splines through HARP data [15], consistent with the latest MiniBooNE publications. In addition, an updated constraint for $K^{+}$production is used as measured by SciBooNE [16]. Fig. 4.4 shows the neutrino flux at MicroBooNE for four neutrino states as modelled by the MiniBooNE beam simulation applied to the MicroBooNE detector.

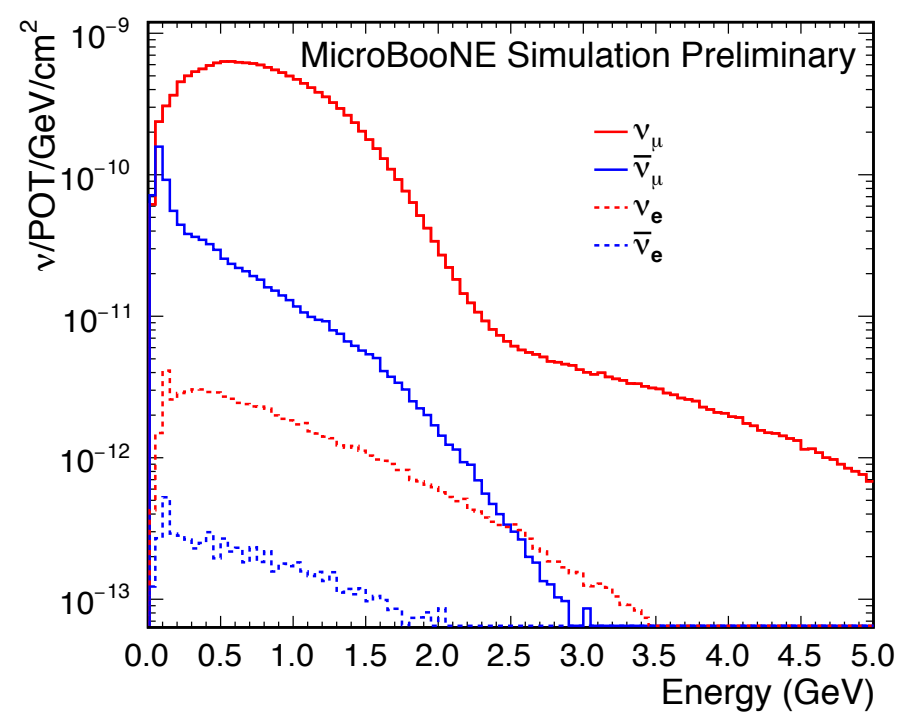

Figure 4.4: Neutrino flux prediction at MicroBooNE for four neutrino states as modelled by the MiniBooNE beam simulation applied to MicroBooNE [2]. 


\section{Chapter 5}

\section{Low Energy Excess: LSND and MiniBooNE}

This chapter provides a historical overview of the low energy excess observed in the LSND and MiniBooNE experiments. In 2001 the LSND experiment reported an excess of $\bar{\nu}_{e}$ events in a $\bar{\nu}_{\mu}$ beam via $\mu^{+}$decay at rest which suggested neutrino oscillations occur in the $\Delta m^{2} \sim 1 \mathrm{eV}^{2}$ range 3 . The MiniBooNE experiment observed an excess of electron like events in a $\nu_{\mu}$ beam at low energy in 2009 [17] which can be interpreted as $\Delta m^{2} \sim 1 \mathrm{eV}^{2}$ oscillations including a single light sterile neutrino. The low energy excess phenomena is explored in this thesis in the MicroBooNE experiment in subsequent chapters.

\subsection{The LSND Signal}

The Liquid Scintillator Neutrino Detector (LSND) experiment ran at Los Alamos National Laboratory from 1993 to 1998 and studied antineutrinos from a decay at rest source of $\mu^{+}$. The source of neutrinos originated from $798 \mathrm{MeV}$ protons impinged on a water target which produced primarily positively charged pions. The positive pions then came to rest and decayed through the sequence,

$$
\begin{aligned}
& \pi^{+} \rightarrow \mu^{+}+\nu_{\mu}, \\
& \mu^{+} \rightarrow e^{+}+\nu_{e}+\bar{\nu}_{\mu},
\end{aligned}
$$


which produced a muon antineutrino with maximum energy $52.8 \mathrm{MeV}$. The LSND detector was a tank filled with 167 tons of liquid scintillator located approximately 30 meters from the neutrino source and surrounded by a veto shield and 1220 8" photomultiplier tubes. LSND searched for $\bar{\nu}_{\mu} \rightarrow \bar{\nu}_{e}$ oscillations via inverse beta decay. Electron antineutrinos were detected by $\bar{\nu}_{e} p \rightarrow e^{+} n$, followed by a neutron capture reaction, $n p \rightarrow d \gamma$, in which a Cherenkov electron signal is followed by a correlated $2.2 \mathrm{MeV}$ photon detection. The antineutrino energy ranged between 20 and $60 \mathrm{MeV}$ with the lower limit imposed to reject $\nu_{e}$ background providing an $L / E \sim 1 \mathrm{~m} / \mathrm{MeV}$. The reconstructed neutrino energy is estimated from the outgoing reconstructed positron energy and angle assuming two body kinematics.

LSND reported $87.9 \pm 22.4 \pm 6.0$ events $[3$ ] consistent with inverse beta decay above expected backgrounds. The excess is shown in Fig. 5.1 as a function of reconstructed positron energy and the ratio of the neutrino interaction length to the reconstructed neutrino energy.
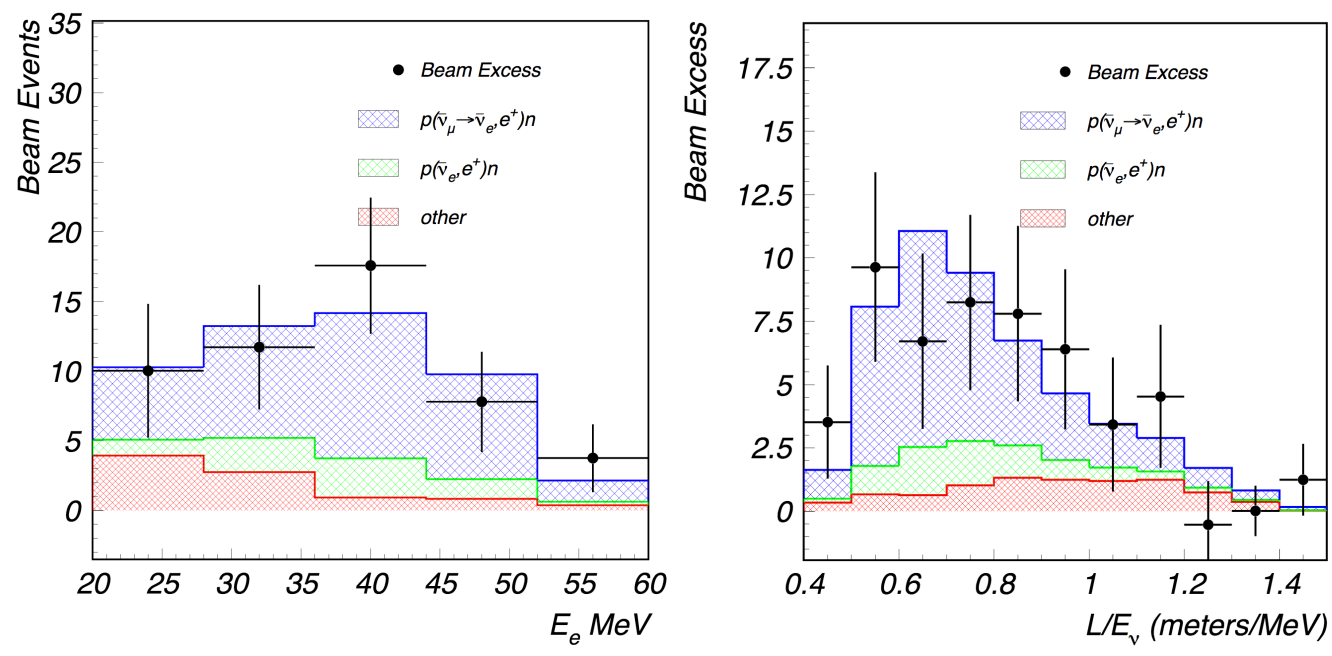

Figure 5.1: Observed LSND data (black points) above expected backgrounds (green and red) and the best fit spectrum for $\bar{\nu}_{\mu} \rightarrow \bar{\nu}_{e}$ oscillations [3] (blue). Left: Data as a function of the outgoing positron energy. Right: Spectrum as a function of the ratio between the distance traveled by the neutrino $L$, and $E_{\nu}$ the reconstructed neutrino energy.

The excess can be interpreted as $\Delta m^{2} \sim 1 \mathrm{eV}^{2}$ oscillations with a mixing angle of $\sin ^{2} \theta_{\mu e}=0.003$. The LSND excess mixing parameters cannot be accommodated by three 
neutrino mixing which allows for two independent $\Delta m^{2}$ scales smaller than $1 \mathrm{eV}^{2}$. One explanation for the LSND excess is the possible existence of sterile neutrino states which cannot interact weakly. The LSND result was addressed by the MiniBooNE experiment which searched for $\nu_{e}$ interactions at a similar baseline and neutrino energy $(\mathrm{L} / \mathrm{E})$.

\subsection{The MiniBooNE Experiment}

\subsubsection{MiniBooNE Detector}

The Mini-Booster Neutrino Experiment (MiniBooNE) detector was constructed to investigate the anomalous excess of electron antineutrino events observed in the LSND experiment. The detector is a spherical Cherenkov detector located at 541 meters downstream of the BNB neutrino production target and has taken a total of $11.27 \times 10^{20} \mathrm{POT}$ in antineutrino mode, and $12.84 \times 10^{20}$ POT in neutrino mode for 15 years of data taking. The detector consists of a 12.2 meter diameter sphere filled with 818 tons of mineral oil and located 3 meters underground and is shown in Fig. 5.2. The active detector region is surrounded by 12808 " photomultiplier tubes, and a $35 \mathrm{~cm}$ thick outer veto region with additional PMTs [18]. The detector is situated in a primarily $\nu_{\mu}$ beam of approximately $750 \mathrm{MeV}$ in energy, the same beam line as MicroBooNE, and resulting in a similar L/E as LSND. The MiniBooNE experiment was designed to search for $\nu_{\mu} \rightarrow \nu_{e}$ and $\bar{\nu}_{\mu} \rightarrow \bar{\nu}_{e}$ oscillations with different backgrounds and systematic uncertainties as an independent check of the LSND observation.

The detection principle is based on Cherenkov light produced by particles passing through mineral oil. Certain final state particles produced in neutrino scattering interactions may attain energies exceeding the Cherenkov threshold. In MiniBooNE, these particles include muons, electrons, and pions. The spatial distribution of Cherenkov light detected by the PMTs located on the detector walls are used for energy, direction, and particle identification. Unique patterns of Cherenkov light are characteristic of the underlying particle which traversed the detector and are shown in Fig. 5.3 .

Three relevant light topologies are shown. The muon particle, characteristic of $\nu_{\mu}$ interactions, emits light radially along its propagation direction producing a pattern of co-centric 

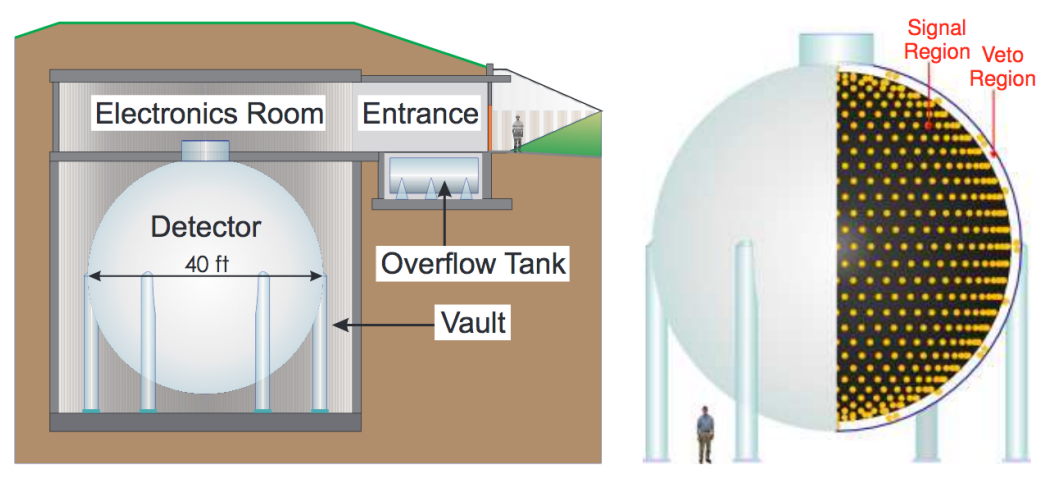

Figure 5.2: The MiniBooNE detector subterranean enclosure (left) showing the experimental hall layout. The PMT configuration inside the tank and in the veto region is show on the right. A human is shown for scale.

\section{Particle Track Cherenkov Ring}

Muon track
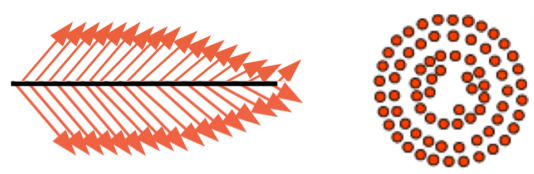

Electron/Photon
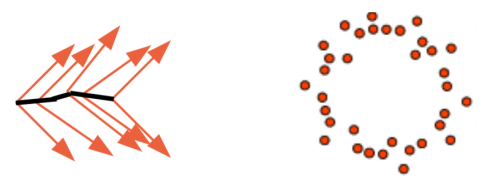

Neutral Pion

(2 photons)
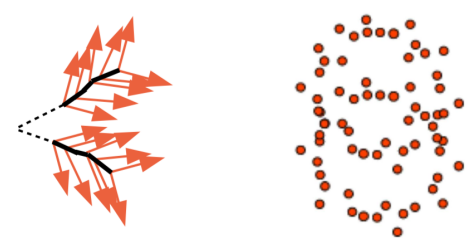

Figure 5.3: Patterns of Cherenkov light characteristic of muon, electron, and neutral pion type particles. The left column shows the light emission behavior along the particle's trajectory while the right column shows the pattern of light observed the photomultiplier array.

circles. The electron, characteristic of $\nu_{e}$ interactions, and the photon particle produces hollow, fuzzy rings. Neutral pions feature two electromagnetic rings with potential for overlap. It is important to note that the electron and single photon ring pattern can not be distin- 
guished which leads to an ambiguity related to the origin of the low energy excess.

The visible particle energy is calibrated using incoming stopping muons which have a well-defined range, as well as electrons from muon decay. Additionally, MiniBooNE made use of $\pi^{0}$ particles to provide energy calibration for the hundreds of $\mathrm{MeV}$ energy scale.

\subsubsection{Event Selection}

The topology of interest in the MiniBooNE oscillation search are charge current quasi-elastic (CCQE) scattering events. CCQE events are identified via an event selection technique which utilizes a maximum likelihood fitting algorithm. The reconstruction algorithm uses features of Cherenkov radiation topologies and PMT timing information to determine the likelihood of particle species. Additional analysis cuts on the distance of the vertex to the detector walls as well as additional kinematics-based cuts remove events which are associated with interactions which take place outside the detector fiducial volume. The CCQE based analysis identified the presence of a single electron or muon particle in time with the beam and with the desired kinematics. The CCQE formula is used to estimate the neutrino interaction energy assuming two body scattering. Both $\nu_{\mu} / \bar{\nu}_{\mu}$ and $\nu_{e} / \bar{\nu}_{e}$ event rates are measured a function of CCQE energy where the measured $\nu_{\mu}$ events are used to constrain the systematic uncertainties on the $\nu_{e}$ as described in Section 10.5. The latest MiniBooNE neutrino data for the $\nu_{e}$ selection is shown in Fig. 5.4 along with the prediction of the background rate from various sources [4].

An excess of events in the data spectrum is observed over the predicted background in the less than $475 \mathrm{MeV}$ region. Above $475 \mathrm{MeV}$ there is good agreement between data and prediction. Multiple sources of background are estimated for the $\nu_{e}$ CCQE search. The primary background in the excess region are photons produced from neutral-current $\pi^{0}$ production and radiative delta decay which are mis-identified as CCQE $\nu_{e}$ interactions. In a $\pi^{0}$ event, one photon may exit the detector, or both photons may overlap with one another, producing an electron like Cherenkov signature. Both sources of photons are constrained by a side band sample of two-gammas observed in MiniBooNE data. Systematic uncertainties on the background prediction are determined by varying the neutrino flux estimates, neutrino cross sections, and uncertainties in detector modelling and reconstruction. In neu- 


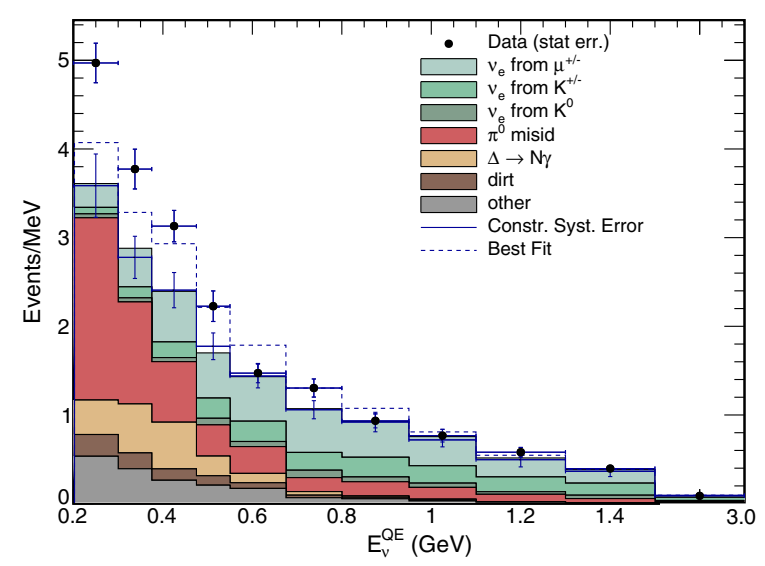

Figure 5.4: MiniBooNE electron neutrino CCQE neutrino mode data for $12.84 \times 10^{20}$ POT data [4]. The stacked colored histogram estimates the background content shown with constrained systematic uncertainties.

trino mode, a total of 1959 data events pass the $\nu_{e}$ CCQE event selection, compared to a background expectation of $1577.8 \pm 39.7$ (stat) \pm 75.4 (sys) events. The excess corresponds to a $4.5 \sigma$ effect. Combining neutrino and anti-neutrino data a $4.7 \sigma$ effect is observed [4].

\subsection{Oscillations Results}

The MiniBooNE event excess data is fit to a two neutrino oscillations model and is shown in Fig. 5.5 to compare with the LSND data. The best fit oscillations point for combined neutrino and anti-neutrino mode is $\left(\Delta m^{2}, \sin ^{2} 2 \theta\right)=\left(0.041 \mathrm{eV}^{2}, 0.92\right)$ and is shown as the black dot. The MiniBooNE $1 \sigma$ allowed regions lies within the LSND $90 \%$ confidence interval demonstrating good agreement between the two signals. Fig. 5.6 shows the event excess for neutrino and antineutrino mode with the best fit oscillations model overlaid. The two neutrino oscillation interpretation of the data requires at least four neutrino types and is indication of physics beyond the standard model in the neutrino sector. The low energy excess is further probed using the MicroBooNE experiment and is the subject of this thesis. 


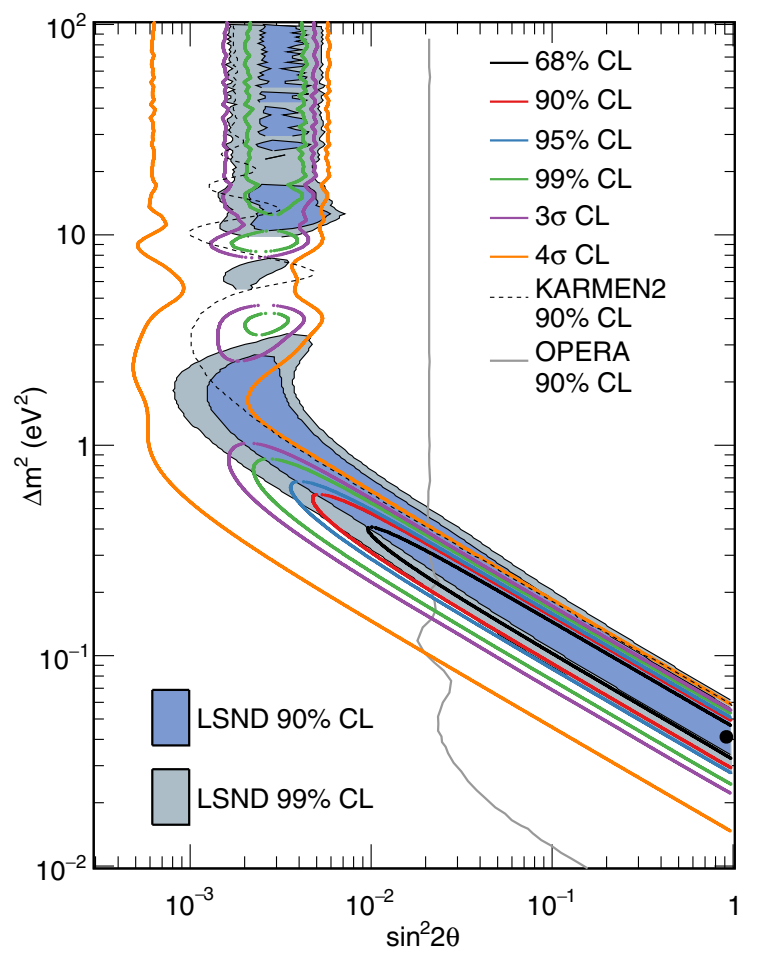

Figure 5.5: MiniBooNE allowed regions for combined neutrino and antineutrino mode running [4. A total of $12.84 \times 10^{20}$ POT and $11.27 \times 10^{20}$ POT data are collected for neutrino and antineutrino mode respectively.

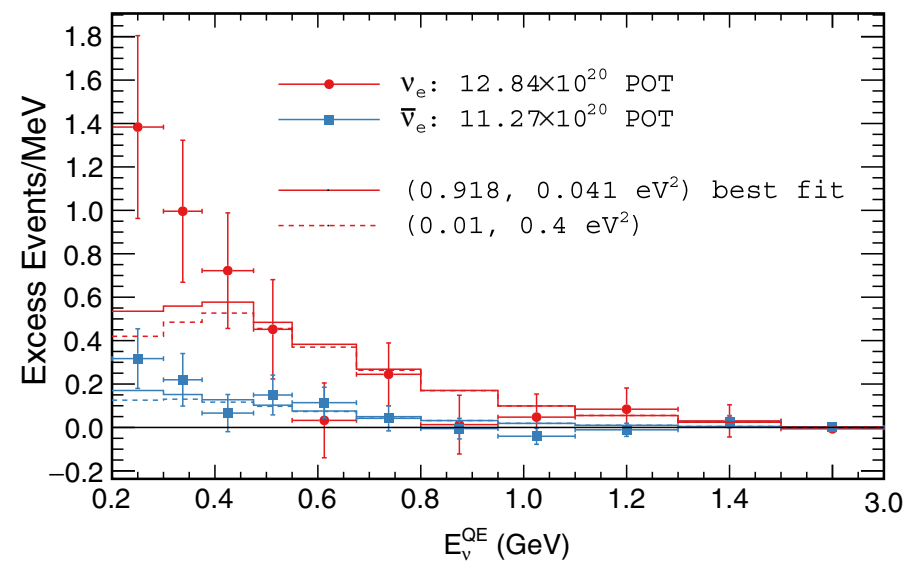

Figure 5.6: MiniBooNE event excess in neutrino and antineutrino mode for the full dataset with statistical and systematic uncertainties [4. The solid (dashed) curve shows the best fit to the neutrino and antineutrino mode data assuming two neutrino oscillations. 


\section{Chapter 6}

\section{Low Energy Excess: MicroBooNE}

This chapter describes the low energy excess signal (LEE) model used to test the MicroBooNE sensitivity to the MiniBooNE low energy excess. The MiniBooNE neutrino mode data is unfolded to estimate the true underlying event excess by removing reconstruction and event selection effects. An electron-like LEE model is described as an energy-dependent modification to the rate of intrinsic $\nu_{e}$ charged current interactions which could appear via a modification of the $\nu_{e}$ flux and/or cross section.

\subsection{MicroBooNE In Context}

The MiniBooNE low energy excess appears as an excess of observed $\nu_{e}$ CCQE events above predicted backgrounds below approximately $600 \mathrm{MeV}$ in reconstructed neutrino energy as shown in Fig. 5.4. In this energy region, a large fraction of background events are non-intrinsic $\nu_{e}$ events as estimated by the MiniBooNE Monte Carlo. These backgrounds include photons coming from neutral current (NC) $\pi^{0}$ events and NC radiative $\Delta \rightarrow N \gamma$ decay events, where $\mathrm{N}$ is a proton or neutron, and are the largest backgrounds in the low energy region where the excess is present. Due to MiniBooNE being a mineral oil Cherenkov detector, it is unable to differentiate Cherenkov rings from electromagnetic showers that originate from either an electron or a photon. Additionally, proton particles do not produce Cherenkov radiation in mineral oil below $1.2 \mathrm{GeV}$ and thus will not be visible in the excess region. The beam excess could be interpreted as an excess of either single photon or 
single electron events, or some combination of the two. A complete picture of the neutrino scattering activity around the vertex cannot be fully reconstructed with the MiniBooNE detector. The MicroBooNE detector, described in detail in Chapter 3, was proposed to elucidate the nature of the low energy excess by allowing for discrimination capability between electron and photon particles as well as provide high resolution information of the neutrino vertex activity.

The MicroBooNE detector is situated in the same neutrino beam at a similar baseline as the MiniBooNE experiment, the MicroBooNE detector is located $470 \mathrm{~m}$ from the BNB production target, while the center of the MiniBooNE detector is $541 \mathrm{~m}$. Two powerful reconstruction techniques are available to discriminate between electron and photon particles in LArTPC detectors. First the $d E / d x$ profile, or charge per unit length, at the beginning of electron and photon showers differs by a factor of two. Electron particles feature an average energy deposition of roughly $2 \mathrm{MeV} / \mathrm{cm}$ (minimally ionizing) before showering. Photon particles feature pair production of an $e^{+} / e^{-}$pair. When the pair is boosted they will overlap and have twice the energy deposition as a single electron. The $d E / d x$ at the beginning of the showers trajectory can be reconstructed by following the charge from the shower start point until its showering point. The $d E / d x$ separation between electron and photon particles has been measured by the ArgoNeuT collaboration [19] and good separation is observed. A selection cut between the expected electron and photon $d E / d x$ of $2.9 \mathrm{MeV} / \mathrm{cm}$ is approximately $76 \%$ efficient for electrons with $7 \%$ photon mis-identification using ArgoNeUT data. Finally, photon particles will invisibly travel approximately $14 \mathrm{~cm}$ in liquid argon until Compton scattering or pair production where charge partciles are produced. In neutrino scattering, electron daughter particles will be connected to the vertex while photon daughter particles, such as in $\pi^{0}$ or $\Delta$ radiative production, will feature a physical gap between the vertex and electromagnetic cascade. The distinction between electron and photon particles can be inferred using the presence of these two features and are included in the neutrino pattern recognition algorithms described in this thesis. With the LArTPC detector technology, MicroBooNE will be able to adress the nature of the MiniBooNE low energy excess. 


\subsection{LEE Prediction in MicroBooNE}

\subsubsection{Unfolding MiniBooNE Data}

The MiniBooNE low energy excess signal is translated into MicroBooNE by unfolding the MiniBooNE observed excess given a model for the signal's origin. The unfolded signal represents the "raw" LEE signal which would be necessary to reproduce the observed excess given the reconstruction efficiency, selection cuts, and detector effects present in the MiniBooNE neutrino mode analysis. There is no model independent way of propagating the excess as the unfolding of the detector effects and reconstruction efficiency is model dependent.

The MiniBooNE observed excess, $O^{\mathrm{MB}}(x)$, as a function of a given observable, $x$, such as reconstructed energy, can be determined by subtracting the MiniBooNE background prediction $B^{\mathrm{MB}}(x)$ from the MiniBooNE observed data $D^{\mathrm{MB}}(x)$,

$$
O^{\mathrm{MB}}(x)=D^{\mathrm{MB}}(x)-B^{\mathrm{MB}}(x)
$$

The observed excess is related to a raw excess prediction in $\operatorname{MiniBooNE}, R^{\mathrm{MB}}(\xi)$, for a given $\mathrm{MC}$ truth level parameter, $\xi$, by

$$
O^{\mathrm{MB}}(x)=A(x, \xi) R^{\mathrm{MB}}(\xi),
$$

where $A(x, \xi)$ is the response matrix. The response matrix encapsulates the detector, reconstruction, and selection effects which can be understood as the conditional probability that a given true parameter $\xi$ ends up in a given reconstructed bin. Equation 6.2 can be solved by inversion and is the process of unfolding. The raw event excess $R^{\mathrm{MB}}(\xi)$ is used to determine the amount by which the MiniBooNE Monte-Carlo central value (CV) predictions would have to be modified in such a way to give rise to an excess consistent with the observation,

$$
M^{\mathrm{MB}}(\xi)=\frac{R^{\mathrm{MB}}(\xi)}{C V^{\mathrm{MB}}(\xi)},
$$

where $M^{\mathrm{MB}}(\xi)$ is the model and $C V^{\mathrm{MB}}$ is the central value in MiniBooNE. The model can then be applied to the MicroBooNE CV, $C V^{\mu \mathrm{B}}(\xi)$, to provide a prediction for the MicroBooNE excess signal, $R^{\mu \mathrm{B}}(\xi)$, for a given model,

$$
R^{\mu \mathrm{B}}(\xi)=M^{\mathrm{MB}}(\xi) C V^{\mu \mathrm{B}}
$$




\subsubsection{Unfolding Methodology}

The unfolding procedure provides a map between a reconstructed variable after the event selection is applied to an underlying true variable, such as true neutrino energy. The problem stated in Equation. 6.2 can be solved exactly using the single value decomposition technique of the response matrix,

$$
A=O S V^{T}
$$

where $O$ and $V$ are orthogonal matrices of square size with dimensions corresponding to the number of reconstructed and true energy bins respectively. The matrix $S$ is a diagonal matrix containing zeroes or positive elements which are called the singular values of $A$. The unfolded spectrum, $u_{\alpha}$, is then given by,

$$
u_{\alpha}=\sum_{k} \sum_{j} V_{\alpha k} O_{k j}^{T}\left(\frac{d_{j}}{s_{k}}\right),
$$

where $d$ is the folded spectrum and $s$ are the singular values of $A$. If any singular values are near or identically zero, statistical fluctuations in the folded spectrum will be amplified in the unfolded spectrum. Instead of directly inverting the response matrix the problem of unfolding is rephrased in terms of finding the solution which minimizes,

$$
(A u-d)^{T} D^{-1}(A u-d)=\min
$$

where $D$ is a covariance matrix. A regularization procedure is applied by introducing a small known bias in order to make an improvement in the variance of the final unfolded spectra. Two methods are used to achieve a regularized unfolded spectrum: Iterative Bayesian Unfolding [20] and SVD Unfolding [21].

The Iterative Bayesian Unfolding procedure is due to D'Agostini and is a widely used approach in the high energy physics community. It is motivated by Bayes theorem and requires a prior guess of the solution which is usually taken to be the Monte Carlo truth or a flat distribution. The initial estimate is then updated via an iterative algorithm to derive the probabilities that a given reconstructed event originated in a true bin at $k+1$. If the procedure is stopped after the first iteration, the resulting spectrum is maximally biased towards the initial estimate. If sufficient iterations are performed, the solution will eventually converge to the matrix inversion solution which contains large variances. In 
between, the solution will contain a non-zero bias with smaller variance. The regularization parameter is taken to be the number of iterations undertaken. The iterative solution for the unfolded spectrum is,

$$
\begin{aligned}
u_{\alpha}^{k+1} & =\frac{1}{\epsilon_{\alpha}} \sum_{i} P(\text { Generated in } \alpha \mid \text { Reconstructed in } i) \\
& =\frac{1}{\epsilon_{\alpha}} \sum_{i}\left[\frac{P(\text { Reconstructed in } i \mid \text { Generated in } \alpha) P(\text { Generated in } \alpha)}{P(\text { Reconstructed in } i)}\right] \\
& =\frac{1}{\epsilon_{\alpha}} \sum_{i}\left[\frac{A_{i \alpha} u_{\alpha}^{k}}{\sum_{\beta} A_{i \alpha} u_{\beta}^{k}}\right] d_{i},
\end{aligned}
$$

where the response matrix $A_{i \alpha}$ is the probability that the event generated in truth bin $\alpha$ is reconstructed in bin $i$.

The SVD Unfolding procedure is mathematically a form of Tikhonov regularization where a penalty term of strength $\tau$ is added to Equation 6.7 to penalize physically implausible solutions for the unfolded spectrum $u$. The derivation can be found in [21]. The exact solution given in Equation 6.6 is replaced with a regularized solution such that,

$$
u_{\alpha}=t_{\alpha} \sum_{k} \sum_{\beta=1} C_{\alpha \beta}^{-1} V_{\beta k}^{\prime} \frac{\hat{d}_{k} s_{k}^{\prime}}{s_{k}^{\prime 2}+\tau},
$$

where $k$ runs over the data spectrum bins, $s_{k}^{\prime}$ represents the singular values of $A$ after a scaling and rotation procedure, and $d$ has been transformed to $\hat{d}$ which has a covariance matrix equal to the unit matrix. The regularization parameter $\tau$ has the effect of allowing for small or zero singular values. Its effects are often likened to a cutoff for a low-pass filter, regularizing the singular effects of small $s_{i}$.

The regularization strength is chosen based on three criteria. First, the unfolded spectra must have as small variance as possible. Second, it must give a bias consistent with zero on the unfolded spectra. Finally, the MiniBooNE data must be consistent with a single experiment drawn from a distribution with expectation value equal to the refolded solution.

\subsubsection{Electron-like LEE Signal Model}

The electron-like signal model is assumed to originate from an energy-dependent modification to the rate of intrinsic $\nu_{e}$ charge current interactions and hypothesized to come from 
a combination of modified cross-section or flux. While not well motived from a theoretical stand point it provides a simple model to test the MicroBooNE sensitivity to an electronlike low energy excess. Under this hypothesis, the MiniBooNE LEE is interpreted as an excess of $\nu_{e}$ events above a background of intrinsic $\nu_{e}$ events and is shown in Fig. 6.1 after subtracting off the other non-intrinsic backgrounds.

MiniBooNE excess, Electron-Like Model

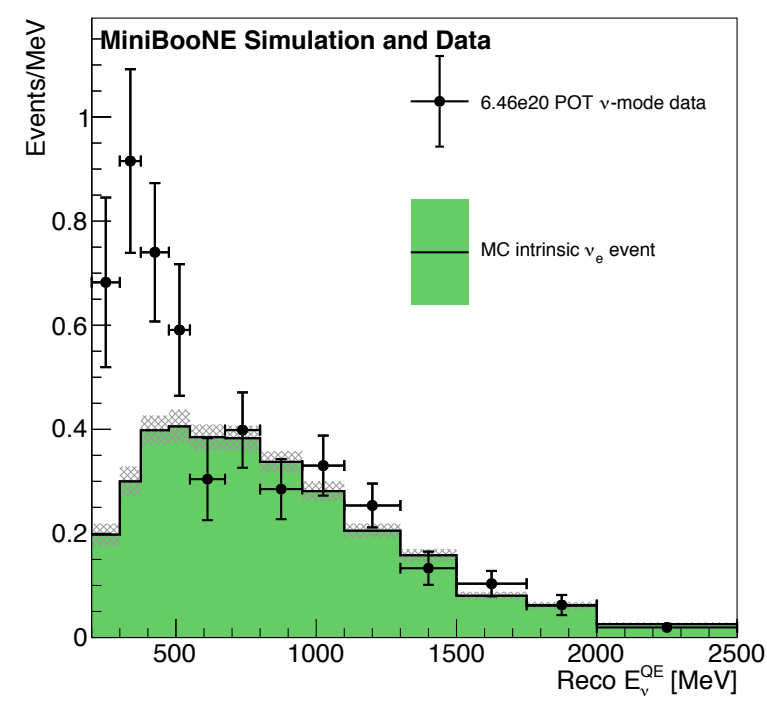

Figure 6.1: The MiniBooNE low energy excess (MiniBooNE data minus predicted background) compared to the absolutely normalized MiniBooNE MC-predicted background originating from intrinsic $\nu_{e}$ events. Statistical uncertainties are shown on the data and MC predictions as the black bars and gray shaded region respectively.

The true underlying energy spectrum is defined as the parent $\nu_{e}$ energy. The reconstructed variable is taken to be the reconstructed CCQE neutrino energy, $E_{Q E}$,

$$
E_{Q E}=\frac{m_{N} E_{\mathrm{vis}}-0.5 m_{e}^{2}}{m_{N}-E_{\mathrm{vis}}+\sqrt{E_{\mathrm{vis}}^{2}-m_{e}^{2}} \cos \theta},
$$

where $m_{N}$ is the mass of the nucleon, and $E_{\text {vis }}$ and $\cos \theta$ are the total energy and angle of the reconstructed electron. The true neutrino energy and reconstructed energy spectrum after the MiniBooNE CCQE event selection are shown in Fig. 6.2 alongside the response matrix $A$.

In the low energy excess region approximately $10 \%$ efficiency is observed. The response 

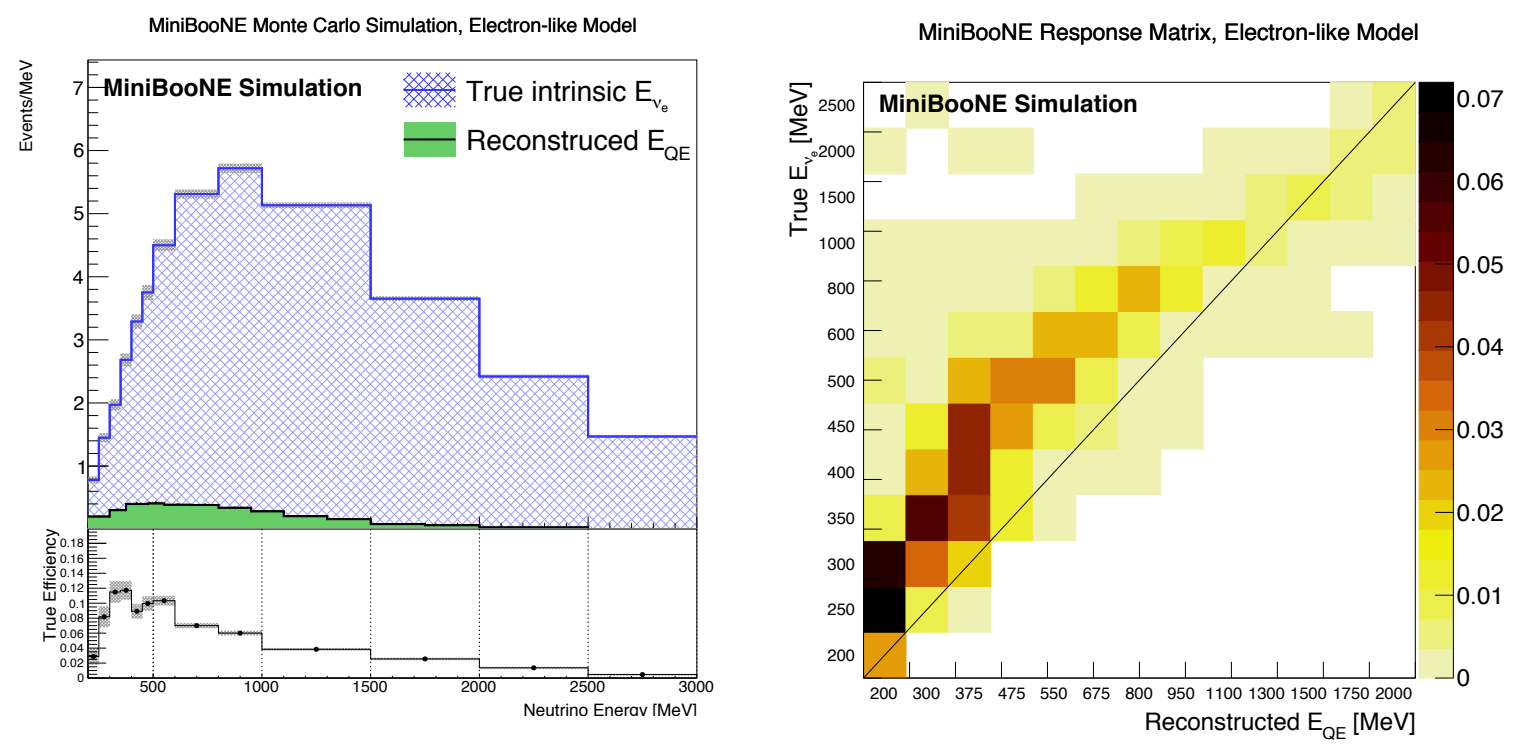

Figure 6.2: Left: True neutrino distribution of intrinsic $\nu_{e}$ events before (blue) and after (green) reconstruction in MiniBooNE. The combined reconstruction and CCQE selection efficiency is shown in the bottom plot. No MiniBooNE MC events are simulated below 200 $\mathrm{MeV}$. Right: The response matrix constructed from the MC truth and reconstructed energy variables. The z scale corresponds to the probability.

matrix shows the smearing of the true neutrino energy into reconstructed energy bins. The unfolding procedure is then applied given the response matrix and is shown in Fig. 6.3 .

The unfolded MiniBooNE spectra shows a strong energy dependence below $600 \mathrm{MeV}$ of true neutrino energy. A factor of 5 increase in the intrinsic $\nu_{e}$ rate is required to reproduce the observed MiniBooNE low energy excess under the electron model. Three iterations of the D'Agostini method are used such that the bias is consistent with zero. As a cross check the SVD Unfolding algorithm is applied and is shown in Fig. 6.4 for the updated $12.84 \times 10^{20}$ MiniBooNE neutrino data [4].

The official signal weights used for the electron-like low energy excess search by MicroBooNE are shown in Fig. 6.5 and represent the factor by which the intrinsic $\nu_{e}$ charge current rate should be scaled to produce the expected signal. 


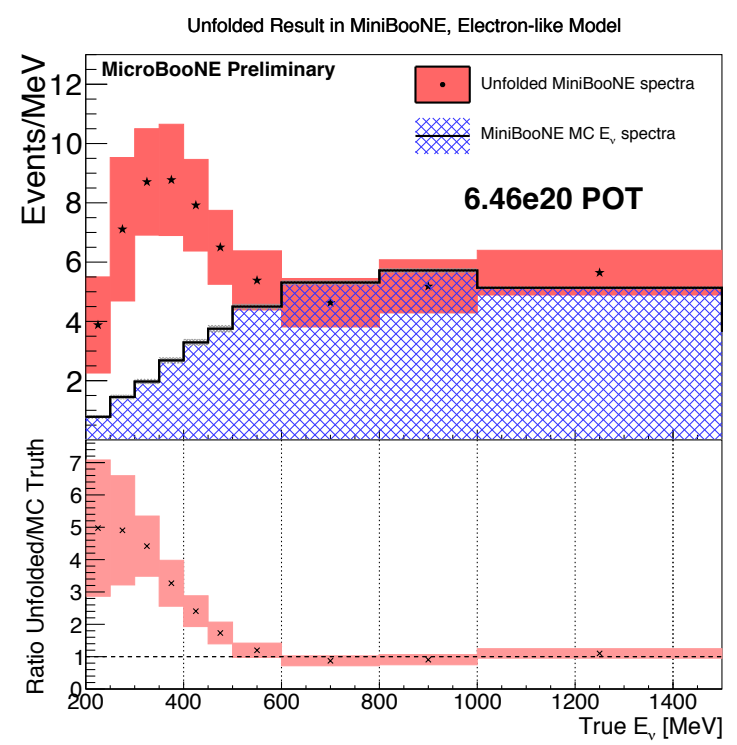

Figure 6.3: Top: MiniBooNE unfolded intrinsic $\nu_{e}$ spectra (red) overlaid on the nominal predicted MC. The unfolded spectrum lies exclusively below $600 \mathrm{MeV}$ in true neutrino energy. Bottom: Ratio of the unfolded to the nominal intrinsic $\nu_{e}$ spectrum with statistical uncertainties only.

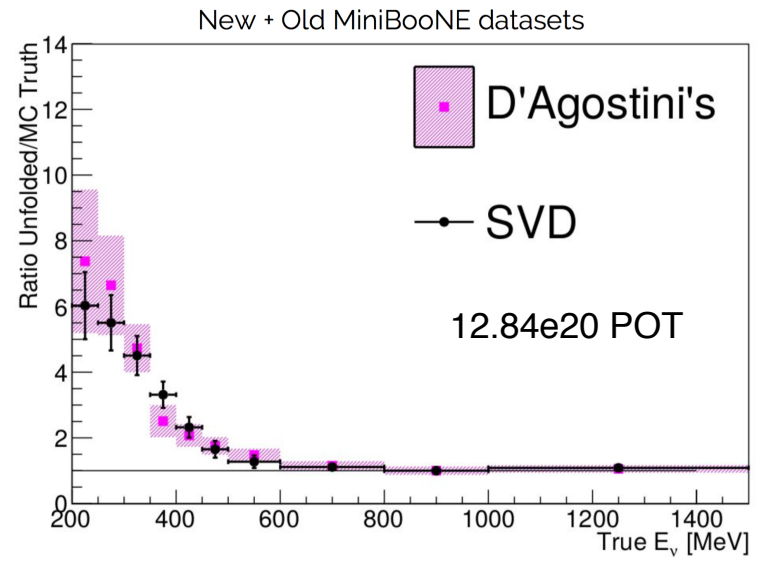

Figure 6.4: Combined ratio of the unfolded to the nominal intrinsic $\nu_{e}$ spectrum from D'Agostini's (purple) and SVD (black) unfolding techniques for the $12.84 \times 10^{20}$ POT MiniBooNE dataset. 


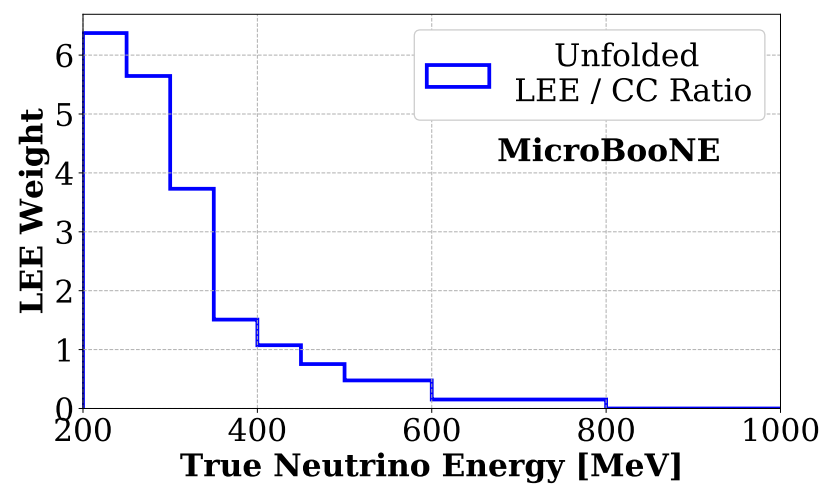

Figure 6.5: The ratio of the MiniBooNE unfolded electron-like LEE model to the intrinsic $\nu_{e}$ event rate in MicroBooNE as a function of true neutrino energy. 


\section{Chapter 7}

\section{Lepton 1 Proton (1L1P) Neutrino Topology}

This chapter will describe the 1 lepton and 1 proton (1L1P) final state topology selected to probe the MiniBooNE electron-like low energy excess. The motivation, and reconstruction and analysis advantages, of the 1L1P topology are discussed. The event rate expectation in MicroBooNE for $13.2 \times 10^{20}$ POT is detailed and the unfolded LEE prediction derived in Chapter 6 is applied. An estimate for expected 1e1P content of the LEE is presented.

\subsection{L1P Signature}

\subsubsection{Motivation}

The majority of the MiniBooNE low energy excess is concentrated in the 200 to $600 \mathrm{MeV}$ true electron neutrino energy range. In this regime the neutrino cross section is dominated by charged current quasi-elastic (CCQE) scattering as shown in Fig. 7.1] (from [22]) and defined for an incident neutrino as,

$$
\nu+n \rightarrow l^{-}+p
$$

In MicroBooNE the neutrino, $\nu$, is primarily of muon or electron neutrino flavor and the outgoing lepton will be either muon or electron. The targets are not free nucleons but rather argon nuclei. The assumption that the neutrino scattering is CCQE remains valid if the 
energy and momentum transfer are large enough to which interaction occurs on individual nucleons. Fermi-motion, binding energy, final state interactions, and other nuclear effects can cause deviations in the final state kinematics away from those expected in free nucleon CCQE interactions.

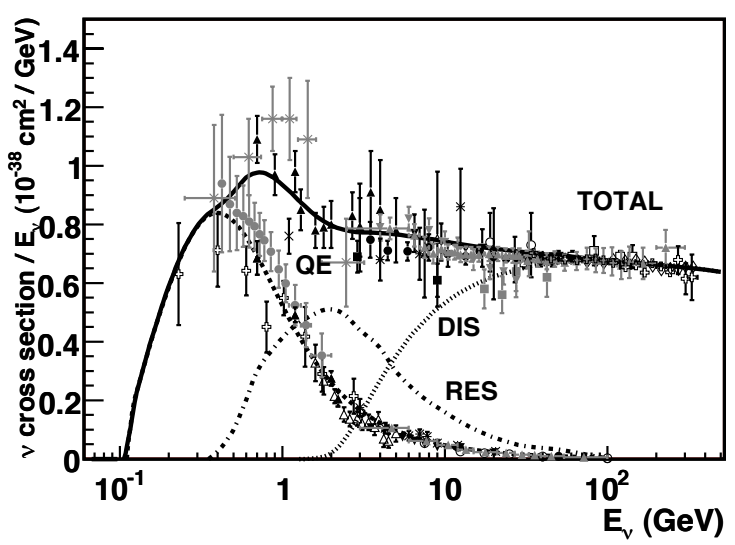

Figure 7.1: Neutrino charged current cross section per nucleon divided by neutrino energy as a function of true neutrino energy. In the low energy region below $600 \mathrm{MeV}$, neutrinos primarily scatter through a quasi-elastic process.

Assuming both the final state electron and proton exit the nucleus, a CCQE interaction in liquid argon features a two prong topology. At the neutrino scattering point two charged particles are emitted, a lepton and a proton. This analysis focuses on identifying generic two prong neutrino topologies, called one lepton one proton (1L1P), to identify low energy CCQE events to investigate the MiniBooNE low energy excess. Electron neutrinos are the primary signal of interest while muon neutrino interactions will be used to constrain the systematic uncertainties on the electron neutrino normalization.

\subsubsection{Topology}

The 1L1P exclusive interaction channel is chosen due to its unique experimental signature in LArTPCs and its high CCQE content in the energy regime of the low energy excess. The 1L1P topology is defined as single lepton and proton in the final state above an energy threshold and contained inside the MicroBooNE TPC. For 1L1P electron neutrino interactions, the lepton emitted is an electron and is called " $1 e 1 \mathrm{P}$ ". Muon neutrino interactions 
feature a muon and are called " $1 \mu 1 \mathrm{P}$ ". Examples of $1 e 1 \mathrm{P}$ and $1 \mu 1 \mathrm{P}$ events are shown in Fig. 7.2 .

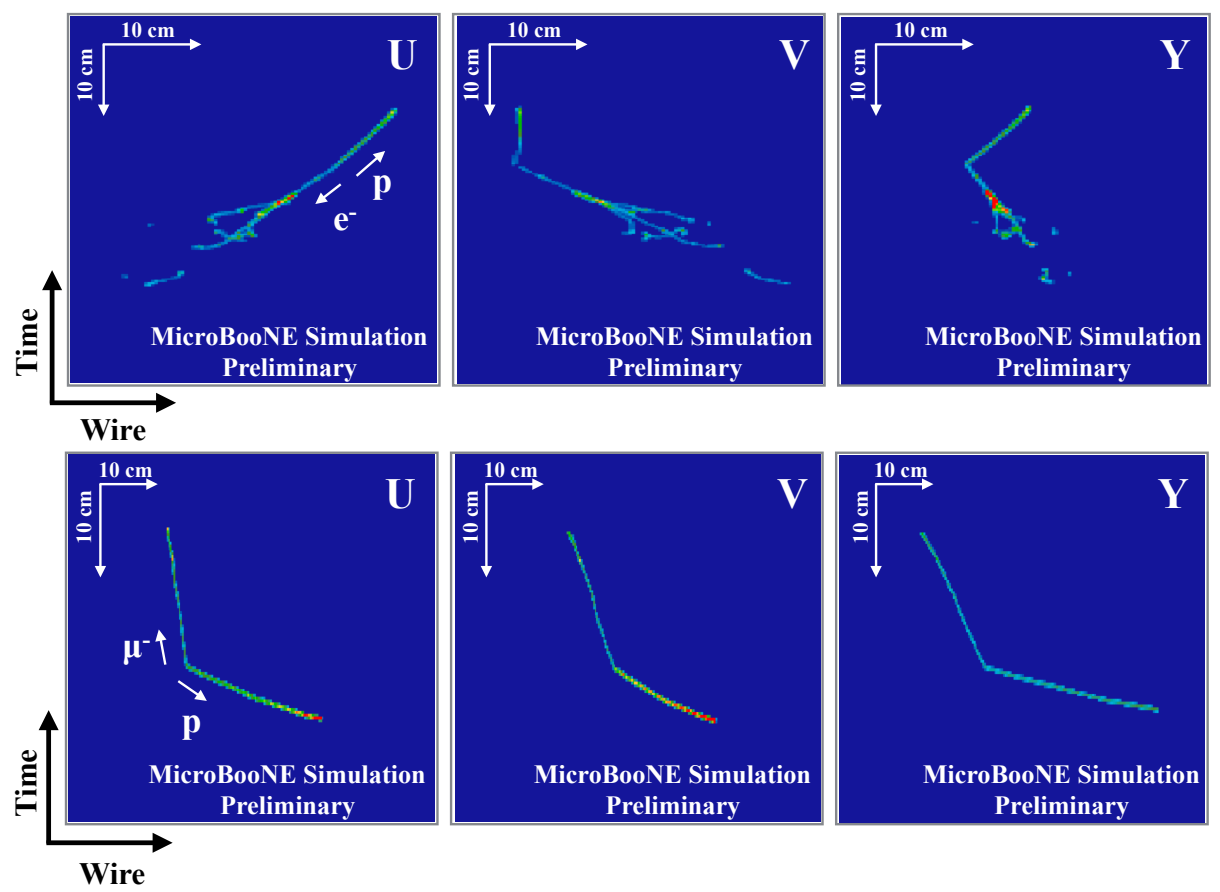

Figure 7.2: U, V, and Y plane projections of 1L1P event topologies represented as 2D images. Top: Example of a 1e1P event in MicroBooNE, where a single proton and electron are produced. Bottom: Example of a $1 \mu 1 \mathrm{P}$ event, where a single muon and proton are produced.

The lepton is used to identify the neutrino flavor. An energy threshold for the lepton and proton particles is applied to ensure a visible ionization pattern in the event. In this analysis, an event is represented by three 2-dimensional images (one per plane) as described in Chapter 8. An energy threshold of $35 \mathrm{MeV}$ is required of the lepton and $60 \mathrm{MeV}$ for the proton and are applied to ensure a two prong feature is observable in the image. For leptons the $35 \mathrm{MeV}$ requirement is reasonable as both the muon and electron will travel appreciable distance in liquid argon at this energy. A $60 \mathrm{MeV}$ requirement on the proton is motivated by visibility of ionization. The distance traveled by protons in liquid argon as a function of kinetic energy is shown in Fig. 7.3

When a proton decreases below 6 wires in length $(1.8 \mathrm{~cm})$ on any one plane the ability 


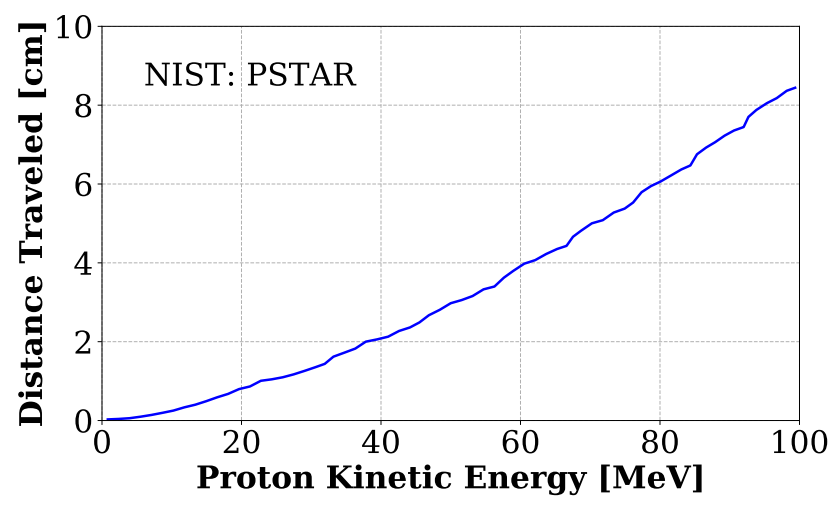

Figure 7.3: Proton range in liquid argon as a function of initial proton kinetic energy. A 60 $\mathrm{MeV}$ proton in a $1 e 1 \mathrm{P}$ interaction traverses a distance of approximately 4 centimeters.

to reconstruct a track becomes challenging. Assuming a proton spans 6 wires in length on an induction plane, the corresponding length on the collection plane is 12 , or $3.6 \mathrm{~cm}$. A proton track of approximately $4 \mathrm{~cm}$ in length corresponds to a proton of $60 \mathrm{MeV}$ kinetic energy. A $60 \mathrm{MeV}$ threshold is set such that the proton spans will span at least 6 wires in its shortest projection. Additionally, any number protons below $60 \mathrm{MeV}$ are allowed in the interaction as they may be invisible. An auxiliary reason to require a visible proton is to reduce the potential for mis-identification with other neutrino channels and cosmic rays. If the proton requirement is removed for the $1 \mu 1 \mathrm{P}$ case $(1 \mu 0 \mathrm{P})$, the single muon emitted from the neutrino interaction must be distinguished from a fierce cosmic ray muon background. For $1 e 1 \mathrm{P}$ events with no proton $(1 e 0 \mathrm{P})$ a single electron must be distinguished against particles produced in cosmic ray showers, and from photon backgrounds such as in neutral current $\pi^{0}$ and radiative $\Delta$ decay where two showers must be identified. The $1 e 1 \mathrm{P}$ topology has the powerful feature of an electron and proton connected at a single point which can be used to reject these types of backgrounds.

The multiplicity and energy thresholds required of the final state topology restrict the fraction of CC neutrino events which can be identified. Fig. 7.4 shows the fraction $\nu_{e} \mathrm{CC}$ events which feature a $1 e 0 \mathrm{P}$ ( 1 electron 0 protons), $1 e 1 \mathrm{P}$, or $1 e \mathrm{NP}$ ( 1 electron $\mathrm{N}$ protons) topology at the generator level.

No other particles such charged or neutral pions are produced in the interaction. In 


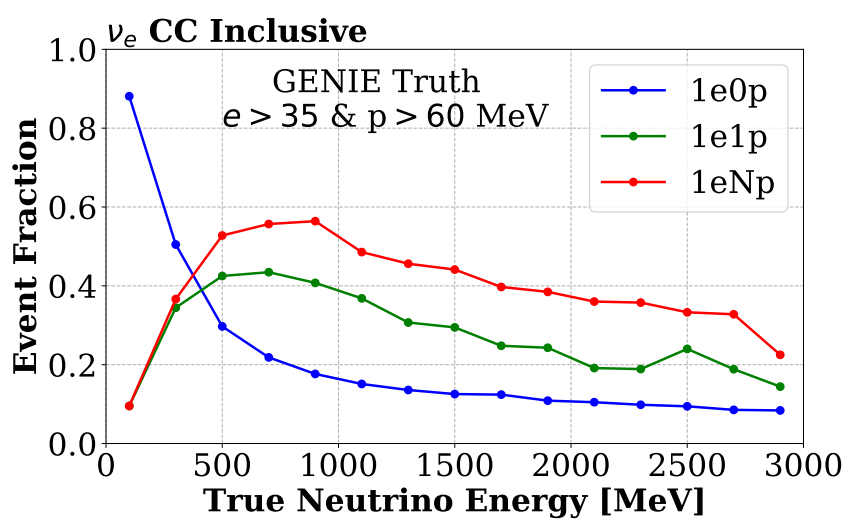

Figure 7.4: Fraction of charged current events which feature a $1 e 0 \mathrm{P}$ (blue), $1 e 1 \mathrm{P}$ (green), or $1 e \mathrm{NP}$ (red) final state topology as a function of true neutrino energy.

the lowest energy bin, $[0,200] \mathrm{MeV}$, approximately $90 \%$ of $\mathrm{CC}$ inclusive interactions feature a single electron in the final state. In the LEE signal region, between $[200,600] \mathrm{MeV}$, approximately $40 \%$ of events are $1 \mathrm{e} 1 \mathrm{P}$. As the true neutrino energy increases, the number of $1 e 1 \mathrm{P}$ events decreases as events with more than one proton, and charged and neutral pions begin to dominate.

The $1 e 1 \mathrm{P}$ topological requirement is a restriction on the visible final state particles rather than a demand on the CCQE interaction channel. The fraction of intrinsic $\nu_{e}$ events per scattering channel which appear as a subset of the signal topology as shown in Fig. 7.5.

Two primary interaction channels are observed in the $[200,600] \mathrm{MeV}$ region, CCQE and meson exchange currents (MEC). MEC interactions, considered a 2 particle 2 hole effect, are characterized by 2-nucleon emission from the scattering vertex, instead of single nucleon emission as in CCQE interactions. The default GENIE model includes MEC using the "empirical", or Dytman, model [23] which is data driven and includes both charged and neutral currents. 1e1P MEC interactions produce an electron with one proton above the 60 $\mathrm{MeV}$ threshold and one proton below. In the low energy region, MEC and CCQE events account for approximately $40 \%$ and $60 \%$ of interactions respectively. Uncertainty on the MEC model is discussed in Chapter 10 . Above $1 \mathrm{GeV}$, resonant and deep inelastic charged current events become more prevalent. In these cases the final state pions and protons are absorbed by the nucleus such that only a single visible electron and proton are visible. 


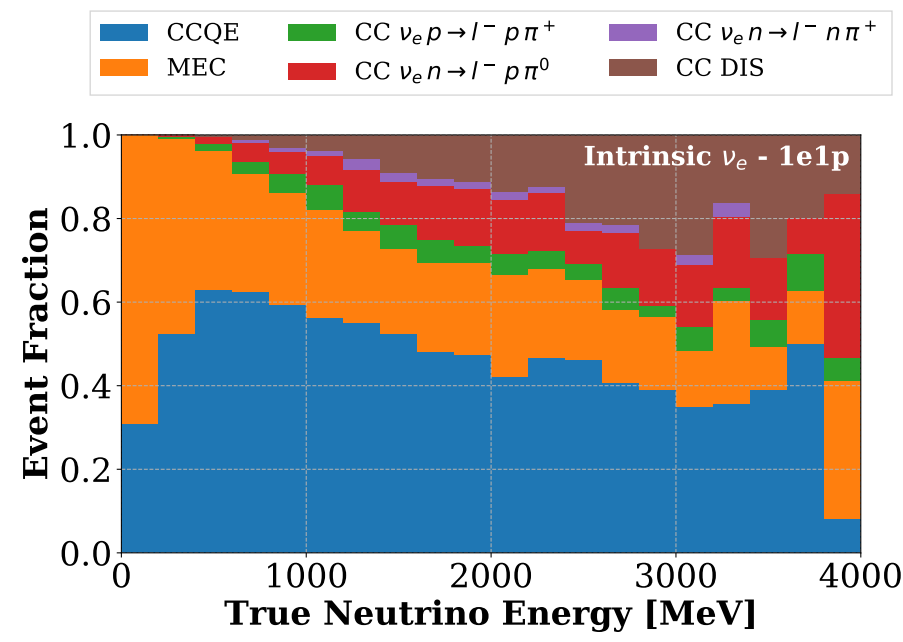

Figure 7.5: Fraction of $1 e 1 \mathrm{P}$ events per true neutrino energy bin which are CCQE (blue), MEC (orange), or other CC (green, red, purple, brown).

\subsubsection{MicroBooNE Event Rate}

The intrinsic electron and muon neutrino event rate at MicroBooNE for $13.2 \times 10^{20} \mathrm{POT}$ is shown in Fig. 7.6 .
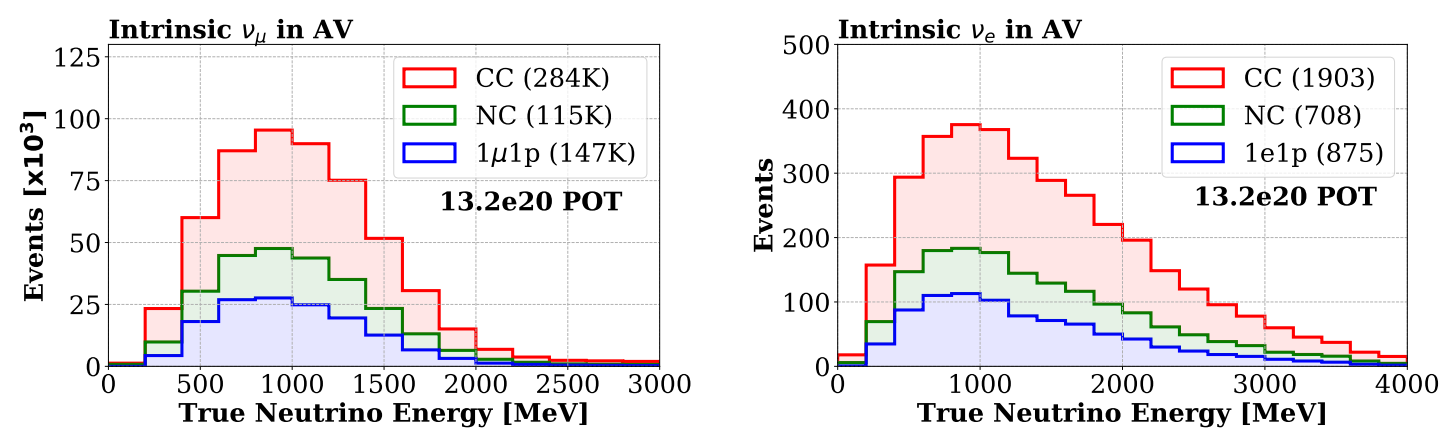

Figure 7.6: Muon (left) and electron (right) neutrino stacked event rate for $13.2 \times 10^{20}$ POT in MicroBooNE for neutrinos contained inside the active volume. The blue distribution shows the 1L1P event rate, the green and red distributions show the rate for neutral current and charge current non 1L1P events respectively.

The event rate is calculated using the GENIE [24] (version 2.12.0) neutrino interaction generator along with the BNB neutrino mode flux outlined in Chapter 4 . In the prediction, 
neutrino interactions are required to scatter inside the TPC active volume such that the interaction is visible in the event image. Neutrino events which occur in the cryostat are not considered. Three modes are shown in Fig. 7.6 in a stacked histogram. The blue distribution shows the 1L1P event rate as a function of true neutrino energy. Approximately 875 events intrinsic $1 e 1 \mathrm{P}$ events are expected over the full energy range. The green and red distributions represent neutral current (NC) and charge current (CC) events which are not 1L1P.

\subsection{LEE Signal Prediction}

An estimate of the electron-like LEE event rate can be made by applying the unfolded MiniBooNE weights. The signal model is the same as detailed in Chapter 6 and is interpreted as an increase the intrinsic $\nu_{e}$ flux or cross section. The weights as a function of true neutrino energy are shown in Fig. 6.4 and are applied to intrinsic $\nu_{e} \mathrm{CC}$ events to form the LEE electron-like rate expectation for $13.2 \times 10^{20} \mathrm{POT}$ in MicroBooNE. The event rate is shown in shown in Fig. 7.7 in $100 \mathrm{MeV}$ energy bins below $1 \mathrm{GeV}$.

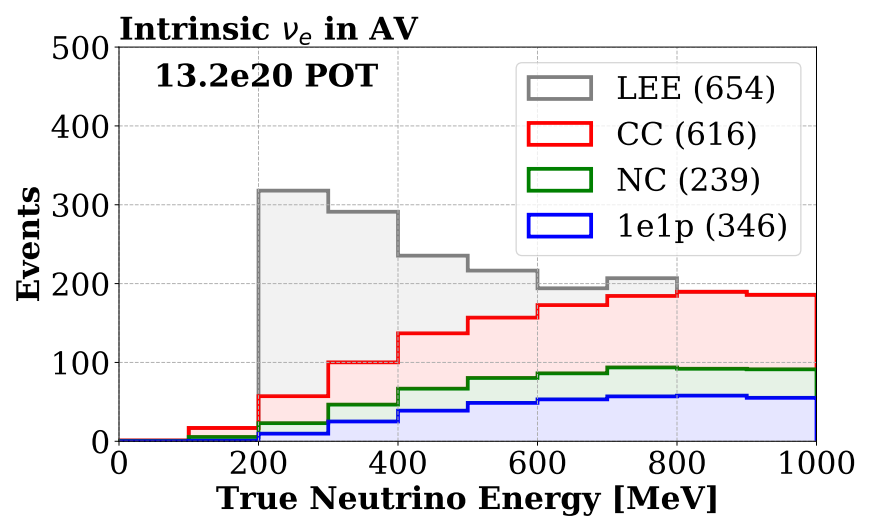

Figure 7.7: LEE signal model (gray) stacked on the 1e1P (blue), NC (green), and CC non $1 e 1 \mathrm{P}$ (red) rate expectations for 13.2e20 POT in MicroBooNE.

Below $800 \mathrm{MeV}$ approximately $654 \mathrm{LEE}$ events are expected of all CC-type interactions, including 1e1p. The $\mathrm{CC}$ (red) and $\mathrm{NC}$ (green) distributions do not exhibit the 1e1P topology and will be significantly vetoed in the reconstruction and analysis detailed in this thesis. The 
1e1P backgrounds to the LEE search are irreducible and are shown in Fig. 7.8, The 1e1P component of the LEE electron-like signal is shown in the gray distribution and represents 192 events over an expected 233 background for $13.2 \times 10^{20}$ POT.

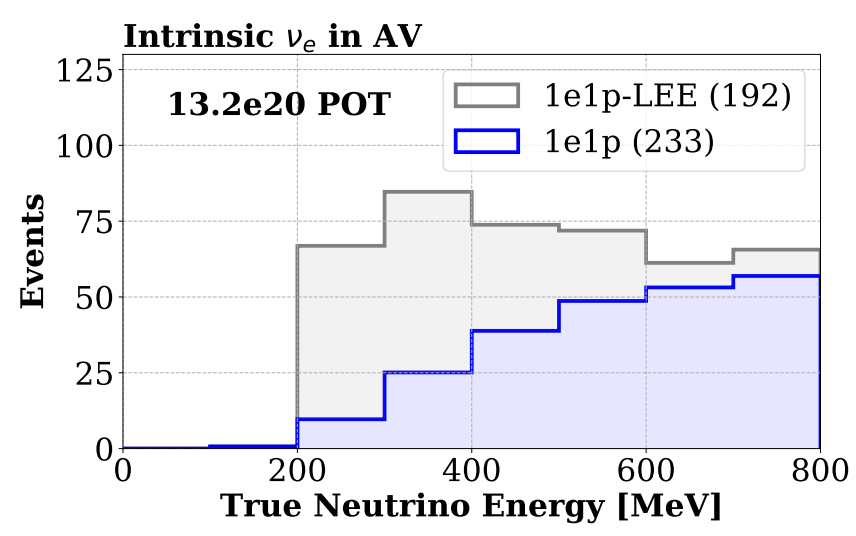

Figure 7.8: The 1e1P component of the LEE signal (gray) stacked on the $1 e 1 \mathrm{P}$ (blue) rate expectation for $13.2 \times 10^{20} \mathrm{POT}$ in MicroBooNE. 


\section{Chapter 8}

\section{Image Based Neutrino Reconstruction}

This chapter describes the automated event reconstruction scheme developed to identify $1 e 1 \mathrm{P}$ and $1 \mu 1 \mathrm{P}$ neutrino interactions in the MicroBooNE detector using TPC and optical data. A novel approach using image based reconstruction techniques is applied to detector data prepared in the form of a 2-dimensional image. Both traditional and deep learning computer vision algorithms are developed for neutrino reconstruction. The event reconstruction chain extracts features in LArTPC images for neutrino detection, daughter particle identification, flavor identification, and momentum determination. After an event is reconstructed both neutrino and background interactions are found in the event. While the reconstruction chain finds neutrino interactions with good efficiency, the neutrino interaction must be discriminated against other backgrounds reconstructed on cosmic ray muons and potentially detector noise. The neutrino selection is described in Chapter 9, An example of the output of the reconstruction chain for $1 \mu 1 \mathrm{P}$ and $1 e 1 \mathrm{P}$ and cosmic background interactions before selection are shown in Fig. 8.1 .

Section 8.1 describes how the LArTPC image is formed from the TPC wire signals and Section 8.2 provides a complete overview of the full image based reconstruction chain. The reader is referred to Sections 8.3 through 8.9 for comprehensive details of reconstruction software components. 

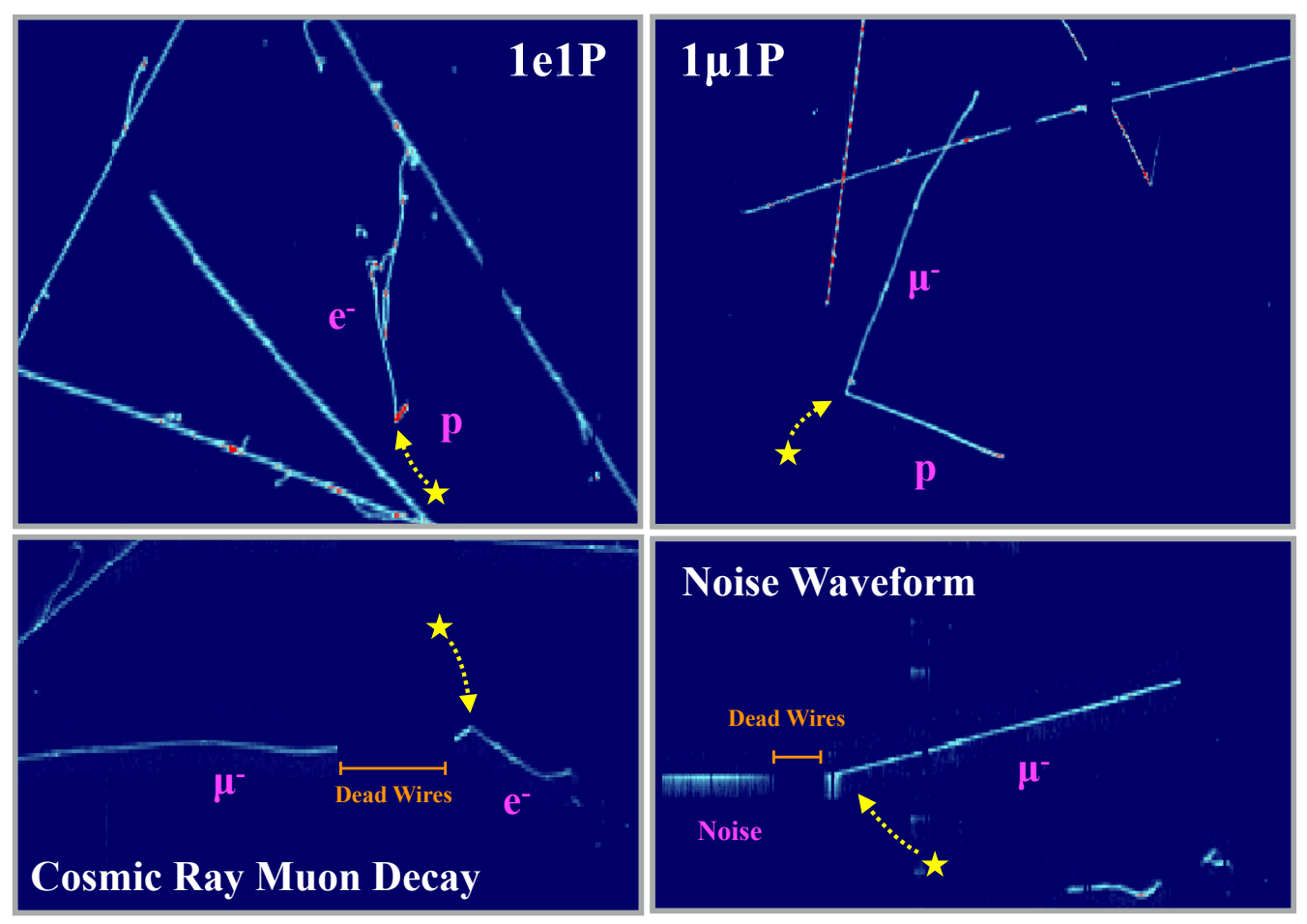

Figure 8.1: Example output of the image based reconstruction chain after 3D vertexing finding for four different types of candidate vertices (yellow star). The neutrino interaction location is identified for $1 e 1 \mathrm{P}$ and $1 \mu 1 \mathrm{P}$ (top row) events along with a number of potential sources of backgrounds (bottom row) including false vertices found on cosmic rays and noise waveforms. The reconstructed particle clusters exiting the vertex are analyzed to determine consistency with electron, muon, and proton particles.

\subsection{Pixelized LArTPC Data Representation}

The event based reconstruction scheme described in this chapter involves MicroBooNE detector TPC data expressed as an image, called a LArTPC image. The two axes of an image are the wire number and readout time. The $\mathrm{Z}$ dimension moves across the detector, upstream to downstream in the neutrino beam direction, while the $\mathrm{Y}$ dimension is a proxy for the $\mathrm{X}$ direction axis. One image per wire plane is formed by filling each column with the digitized waveforms from each wire. In such images, one pixel corresponds to $0.55 \mathrm{~mm}$ along the time axis given the measured drift velocity of $0.11 \mathrm{~cm} / \mu \mathrm{s}$ [25]. The pixel values of the image represent the ADC charge on the wire at the given time tick after noise-filtering 
and signal deconvolution. This scheme produces high resolution images for each wire plane with full charge resolution. In this analysis, the LArTPC image is compressed in the time dimension by a factor of 6 by summing adjacent pixels. The compression factor is chosen to normalize the $\mathrm{X}$ (wire) and $\mathrm{Y}$ (time) dimensions such that they represent approximately the same physical scale. Each pixel represents an approximately $0.3 \mathrm{~cm}$ square projection. The down sampled image is physically smaller which is beneficial for persistent storage and for development of pattern recognition algorithms described in the coming sections.

Three LArTPC images are formed per event with the $\mathrm{U}$ and $\mathrm{V}$ induction planes carrying an image dimension of size $2400 \times 1600$ pixels and the Y collection plane of size 3456 x 1600 pixels. The charge information is stored in a 4 byte floating point pixel value. Images are stored on disk using the ROOT [26] file format using a custom developed software and image representation called Liquid Argon Computer Vision (LArCV) [27]. The LArCV software is integrated into the MicroBooNE production pipeline allowing for both real and simulated data to be converted to the appropriate image format for analysis. Two representations of LArTPC images are used in this chapter. The first image representation is called the LArCV image which contains the down sampled image as described above. This image is used in persistent storage and for interfacing with Convolutional Neural Network (CNN) frameworks to extract machine learned features. The second type of image is called a Liquid Argon Open-source Computer Vision (LArOpenCV) [28] image. An LArOpenCV image is a gray scale representation of the LArCV image and is encoded in a compressed data structure where the pixel value is encoded in a single byte. The conversion between the $\mathrm{LArCV}$ and LArOpenCV image is done by thresholding the LArCV image at 10 pixel count, and assigning pixel values larger than 1 byte to 255, effectively compressing the charge information. The LArOpenCV image allows for interfacing with the Open-source Computer Vision (OpenCV) [29] library as they share a common image data structure. This image is primarily used for application of custom traditional computer vision techniques to extract hand-designed features. 


\subsection{Summary of Image Based Reconstruction}

In this section an overview of the reconstruction chain developed to identify 1L1P events in LArTPC images is presented for readability. An extended description of the methodology of the reconstruction algorithms and a profiling of the reconstruction performance are detailed in the following sections.

\section{PMT Precuts - Section 8.3}

Event images in MicroBooNE can be complex, with on average 15 particles, mostly muons, crossing the detector along with the potential presence of a neutrino interaction. An example full resolution LArTPC event image, from MicroBooNE, of the collection plane is shown in Fig. 8.2 ,

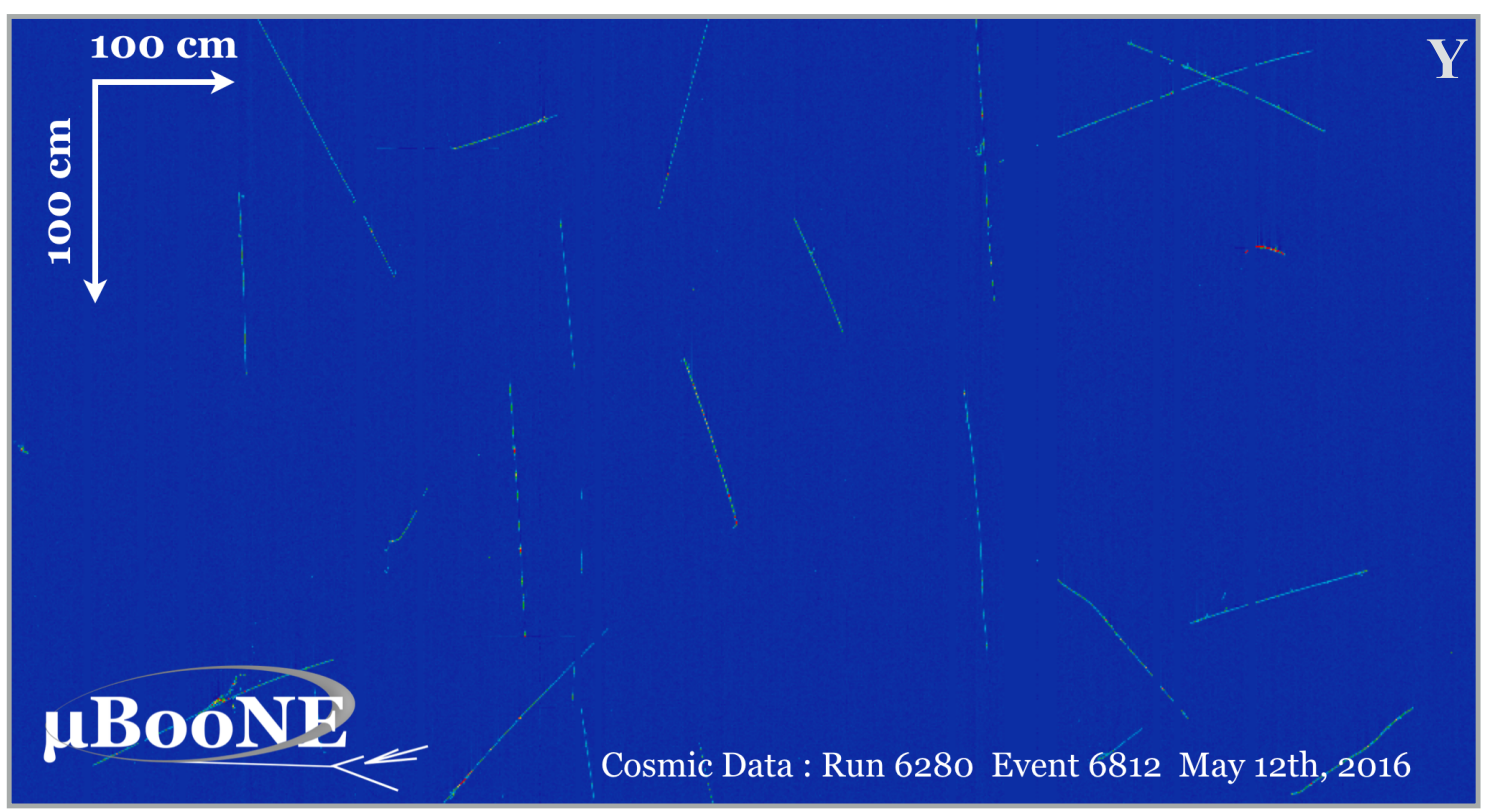

Figure 8.2: Example LArTPC data image of raw signal from MicroBooNE's collection plane. Cosmic muons are observed traversing the detector. Event comes from EXT-BNB trigger. No analysis selection is applied to select this event.

In this image, cosmic ray muons can be seen traversing the detector volume while the rest of the image is empty. Sifting through the charge depositions in the image for the neutrino 
interaction is challenging because of the high cosmic ray rate due to MicroBooNE's position on the Earth's surface. In MicroBooNE, the expected cosmic to cosmic and neutrino event ratio is approximately 600 to 1 . After applying the software trigger [30] that looks for scintillation light coincident with the expected beam window, this ratio is around 30 to 1. This means on average there will be approximately 450 cosmic muons per neutrino interaction.

To reduce the reconstruction load for TPC based 1L1P reconstruction an optical filter, called the PMT precuts, is designed to remove events with no likely neutrino flash. The precuts reject events with light in the beam spill window that are due to low energy backgrounds as well as some additional cosmic ray activity not removed via the software trigger. Cosmic ray tracks themselves are difficult to isolate using only optical information and are removed using features derived from downstream pattern recognition algorithms. After applying the PMT precuts, an additional $75 \%$ of cosmic ray only events are rejected. When applied to neutrino events the precuts are approximately $97 \%$ efficient for retaining $\nu_{\mu}$ and $\nu_{e} \mathrm{CCQE}$ interactions.

\section{Cosmic Ray Tagging and Contained Region of Interest - Section 8.4}

With the cosmic only image rate reduced, a pixel level tagging algorithm is designed to identify cosmic ray trajectories in the image. This TPC based reconstruction algorithm is called the cosmic tagger, and used to identify two classes of cosmic ray tracks: throughgoing muons, and stopping muons. An illustration of the three types of objects found in the event image are shown in Fig. 8.3 .

Reconstructing cosmic rays before applying neutrino finding algorithms is simpler for two reasons. First, cosmic rays trajectories are predominantly long straight tracks and have a simple topology in a LArTPC image. Secondly, additional feature information is available from the TPC and optical system for particles which interact with the detector boundaries. To identify the two classes of cosmic ray tracks, the boundary points at the six detector faces are reconstructed. Each boundary point has a unique signature which can be identified using a combination of the spatial location of TPC and optical signal.

Through-going cosmic ray muons will cross the boundary of the active region of the 


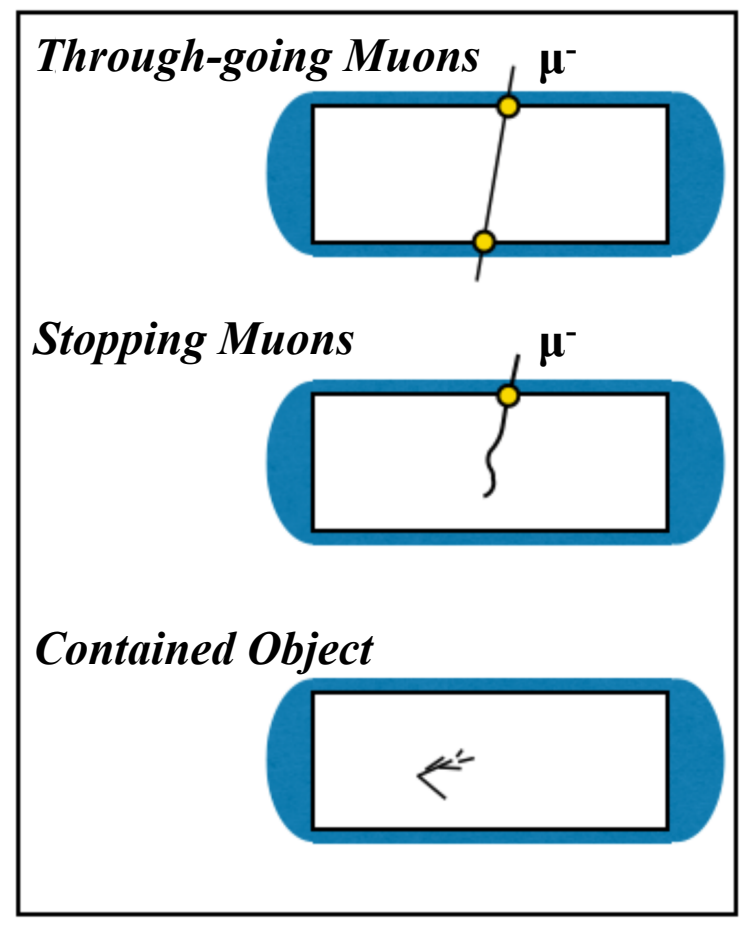

Figure 8.3: Three classes of pixel type reconstructed by the cosmic ray tagger. Throughgoing muons enter and exit the TPC boundary, stopping muons enter the TPC and stop inside, and contained objects whose cluster is completely contained within then TPC boundaries.

detector twice, at an entering and exit point, then follow a path that is fairly close to a straight line. A two stage 3D tracking algorithm is developed to follow pair-wise combinations of boundary points through the detector. If the tracking algorithm connects one boundary point to the other while following pixels in the image, a through-going muon is identified.

Stopping muons are reconstructed by following typically a curved path (due to multiple coulomb scattering) of charge from exactly one point on the TPC boundary into the detector. The 3D tracking algorithm follows the track from the detector boundary into the TPC until a termination point is identified. A neutrino event, particularly high energy $1 \mu 1 \mathrm{P}$ events, may have exiting particles and will have pixels that cross a boundary. In that case, the neutrino event will be tagged with the same flag as a stopping particle. Additional 
pattern recognition of stopping particle pixels is required to determine the tracks origin as either cosmic or neutrino induced.

With through-going and stopping muon tracks identified, the remaining charge deposition in the image should not cross the detector boundary and are labelled as contained. Contained charge may be debris from entering neutral particles of cosmic origin or may be neutrino interactions. Tagging cosmic ray tracks simplifies the image for pattern recognition of contained charge. In this reconstruction, tagged through-going muon pixels are removed from the event image before the neutrino interaction reconstruction step. The stopping muon tracking algorithm is turned off. In simulated events of CORSIKA [31] cosmic rays an average of $75 \%$ of cosmic ray pixels are tagged. Given perfect boundary point reconstruction, an average of $90 \%$ of cosmic pixels can be tagged.

Due to the large size of a LArTPC image an algorithm is used to isolate a contained region of interest, or cROI, where the neutrino interaction is likely to occur. The in-time optical flash is used to set the spatial location of the cROI to reduce the search region for the neutrino interaction. The size of the cROI corresponds to approximately one third the size of the event image. On a sample of simulated neutrino events, the efficiency for placing the cROI on the image which covers the neutrino interaction is $95 \%$. Inside the cROI, a hybrid of traditional and deep learning algorithms are applied to reconstruct the neutrino interaction.

An example output of the cosmic ray tagging and cROI finding algorithm is shown for an example MC intrinsic $\nu_{e}$ with CORSIKA cosmic rays in Fig. 8.17. 


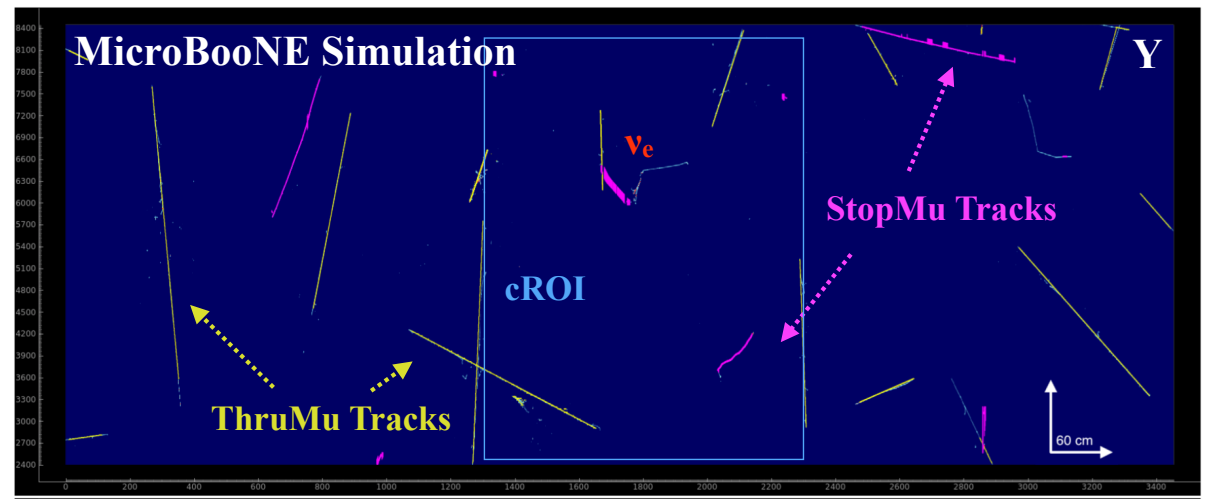

Figure 8.4: Output of the cosmic ray tagging and cROI finding algorithm. Three objects are identified: through-going muon tracks (yellow), stopping muon tracks (pink), and a contained region of interest (blue) to search for a neutrino interaction. 


\section{Track and Shower Pixel Tagging using Semantic Segmentation - Section 8.5}

To identify the presence of a 1L1P neutrino interaction in the cROI, a proton, and either a muon or electron particle must be identified having topological and kinematic features consistent with neutrino scattering. To aid in the reconstruction of the neutrino scattering point and provide a useful feature for particle identification, a deep learning based algorithm is developed to identify track and shower pixels. Two particle topologies, like 1L1P, are composed of two basic LArTPC features: the presence of two track particles in the case of $1 \mu 1 \mathrm{P}$ or the presence of a single track and shower particle in the case of $1 e 1 \mathrm{P}$. Tracks and showers are defined by their unique ionization pattern. Tracks are LArTPC image features consisting primarily of lines left behind by heavy charged particles that travel through the detector such as muons, pions, and protons. Shower topologies consist of a collection of branching features which are produced when electrons propagate through the detector and initiate an electromagnetic cascade of particles. Distinguishing between track and shower topologies is the simplest form of high level particle identification, and can be a powerful discriminant to distinguish between electron and muon neutrino interactions in LArTPC detectors. Additionally, track and shower pixel labelling enables a more efficient search for the $3 \mathrm{D}$ neutrino interaction vertex as assumptions can be made about the nature of pixels being used for pattern recognition.

A deep neural network has been trained for a semantic segmentation task, called SSNet [8]. The SSNet deep learning algorithm is applied inside the cROI and assigns a score of being track-like or shower-like to every pixel. An example of input and output to the SSNet is shown in Fig. 8.5. A pixel-wise classification, called semantic segmentation, provides a novel algorithm for identifying shower particle features which originate from a stochastic nature of shower evolution. When applied to images of low energy 1L1P events, the SSNet shows good performance at pixel classification. On average, $2.3 \%$ and $3.9 \%$ of charge carrying pixels are mis-classified for low energy $1 \mu 1 \mathrm{P}$ and $1 e 1 \mathrm{P}$ events respectively.

\section{Vertex Finding and Particle Clustering - Section 8.6}

Vertex activity is an evidence of neutrino scattering activity in liquid argon detectors. The vertex is the detector location where the neutrino scatters off an argon nucleus and 


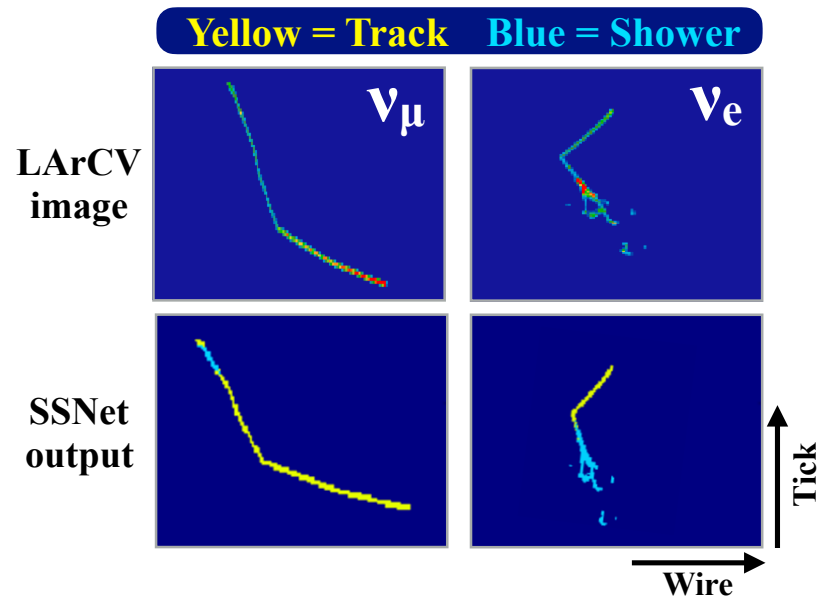

Figure 8.5: Example $1 \mu 1 \mathrm{P}$ and $1 e 1 \mathrm{P}$ SSNet input (top) and output (bottom). A LArCV image containing the particle ionization pattern, called an ADC image is used as input to SSNet. The network outputs an image of the same spatial dimension with track (yellow) and shower (cyan) pixels labelled.

where electron, muon, and proton neutrino daughter particles begin their ionization trajectory. This reconstruction step searches for the $3 \mathrm{D}$ vertex and clusters pixels belonging to individual particles inside the cROI.

In 1L1P scattering two particles are emitted from the interaction point. Both electron and muon particles will be physically connected to a proton at a vertex. The particles form a "vee" shape, or an angular feature between the lepton and proton projected trajectories. The vertex algorithm searches for a coincident vee shape across two or more planes to claim a 3D consistent vertex candidate. Two separate vertexing algorithms are used to find vertices for two track, called a track vertex, and for one track one shower, called a track-shower vertex, topologies.

Track vertex reconstruction is applied to track pixels labelled by the SSNet and identifies the vertex for $1 \mu 1 \mathrm{P}$ interactions. Both the muon and proton are particles which feature track, or straight line, features in a LArTPC image. To locate the $3 \mathrm{D}$ vertex, $2 \mathrm{D}$ vertex "seeds" are identified on each plane using a kink finding algorithm. In each plane image a collection of vertex seeds are found using a suite of traditional image reconstruction techniques which may represent $3 \mathrm{D}$ projections of a $3 \mathrm{D}$ vertex. The set of $2 \mathrm{D}$ vertex seeds 
is reduced by executing an exhaustive scan of pixels locally around each seed to best locate the kink point as a function of time tick using a template feature which minimizes the angular deviation, called the angular metric, of tracks exiting a circle. The time profile of the angular deviation of tracks is compared across the 3 wire planes to determine the presence of a coincident kink point. A minimizer is then used to scan the time profile and locate candidate $3 \mathrm{D}$ vertex points. The $\mathrm{X}$ position of the vertex is determined from the time coordinate, and the $\mathrm{Y}$ and $\mathrm{Z}$ positions are determined from the coincidence of wires between planes. When applied to $1 \mu 1 \mathrm{P}$ events, vertexing finding is approximately $60 \%$ efficient at locating the true neutrino vertex with $68 \%$ of vertices being reconstructed within 0.9 centimeter of the true interaction vertex.

Track-shower vertex finding is applied to the combination of track and shower labelled pixels by SSNet and is used to identify 1e1P interactions. Applying the track vertex algorithm to the combined track and shower image would not be productive as the typical electron features an electromagnetic cascade containing many kink points which could fake a candidate vertex location. The track-shower vertex algorithm locates the presence of a shower on a track edge. The track edge point is then correlated across planes. If a 3D correlation exists, a grid search is applied to a defined 3D region around the edge point. The angular metric is minimized across planes to determine the optimal candidate location. The $\mathrm{X}, \mathrm{Y}$, and $\mathrm{Z}$ coordinates are determined following the same procedure as in the track vertex case. When applied to $1 e 1 \mathrm{P}$ events, vertexing finding is approximately $53 \%$ efficient at locating the true neutrino vertex with $68 \%$ of vertices being reconstructed within 1 centimeter of the true interaction vertex.

The output of the algorithm for track and track-shower vertex finding is shown in Fig. 8.6 as applied to an example $1 \mu 1 \mathrm{P}$ and $1 e 1 \mathrm{P}$ event.

Finally, each outgoing particle is clustered uniquely by an algorithm for downstream analysis. The complexity of particle clustering is reduced as each particle is assumed to originate from the candidate vertex location. Particles clustered at the vertex are matched across planes to determine 3D consistency. At least 1 particle must be matched across planes to claim a candidate vertex. 

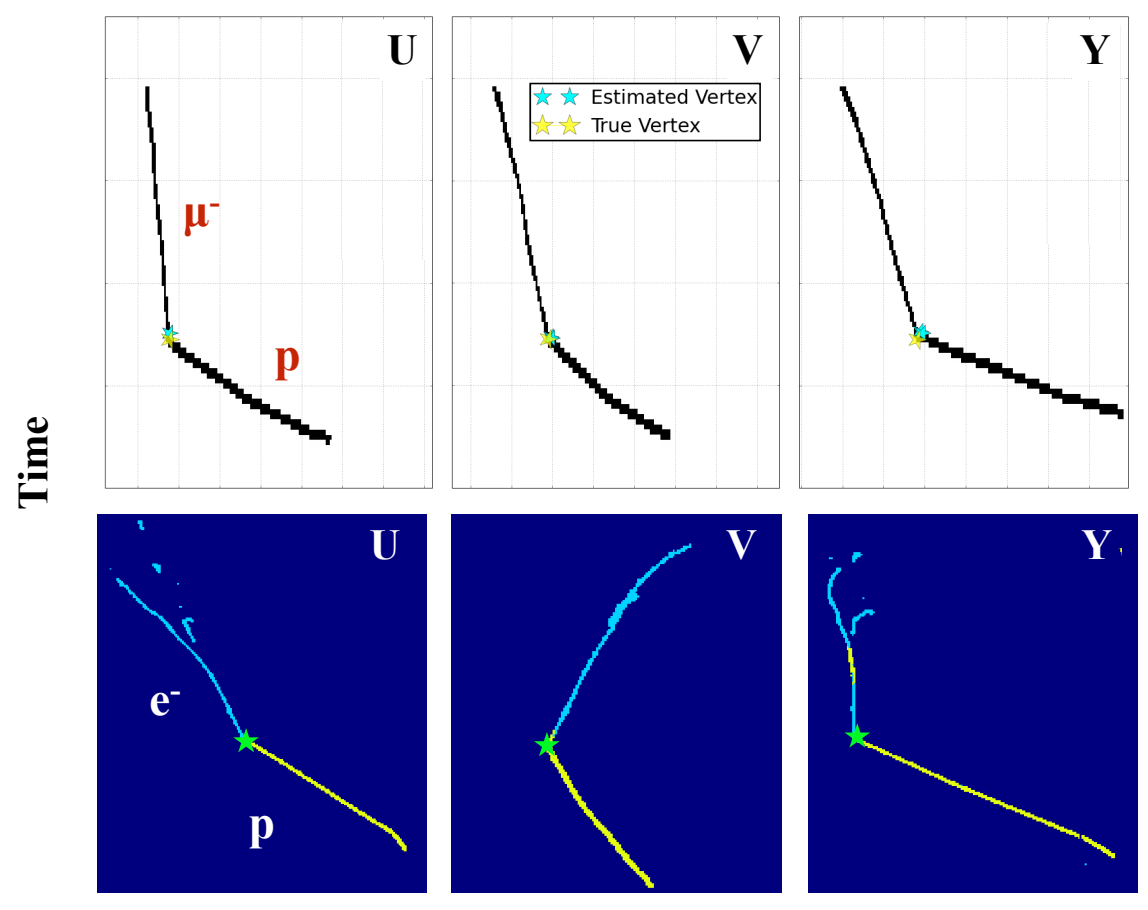

Wire

Figure 8.6: Example of vertex finding applied to an example $1 \mu 1 \mathrm{P}$ (top) and $1 e 1 \mathrm{P}$ event (bottom). Top: A vertex is found (cyan star) close to the true vertex (yellow star) in the track only image (black pixels). Bottom: A track-vertex is found (green star) at the interface between track (yellow) and shower (cyan) pixels.

\section{$1 \mu 1 \mathrm{P}$ Reconstruction - Section 8.7}

A tracking algorithm based on a stochastic search is used to determine the 3D trajectories of particles produced in $1 \mu 1 \mathrm{P}$ interactions. The algorithm steps in $3 \mathrm{D}$ starting at the vertex point along a consistent path of charge until a particle's end point is reached. An example of the algorithm applied to a $1 \mu 1 \mathrm{P}$ interaction is shown in Fig. 8.7 .

The reconstructed trajectory of a track provides a measurement of a particle's 3D length as well as an understanding of a particle's scattering profile which can be used for pattern recognition. For contained muon and proton particles the 3D length can be translated into a kinetic energy which can then be used to estimate the neutrino energy of the interaction. A range based energy estimation method avoids calibration of the energy scale using the 

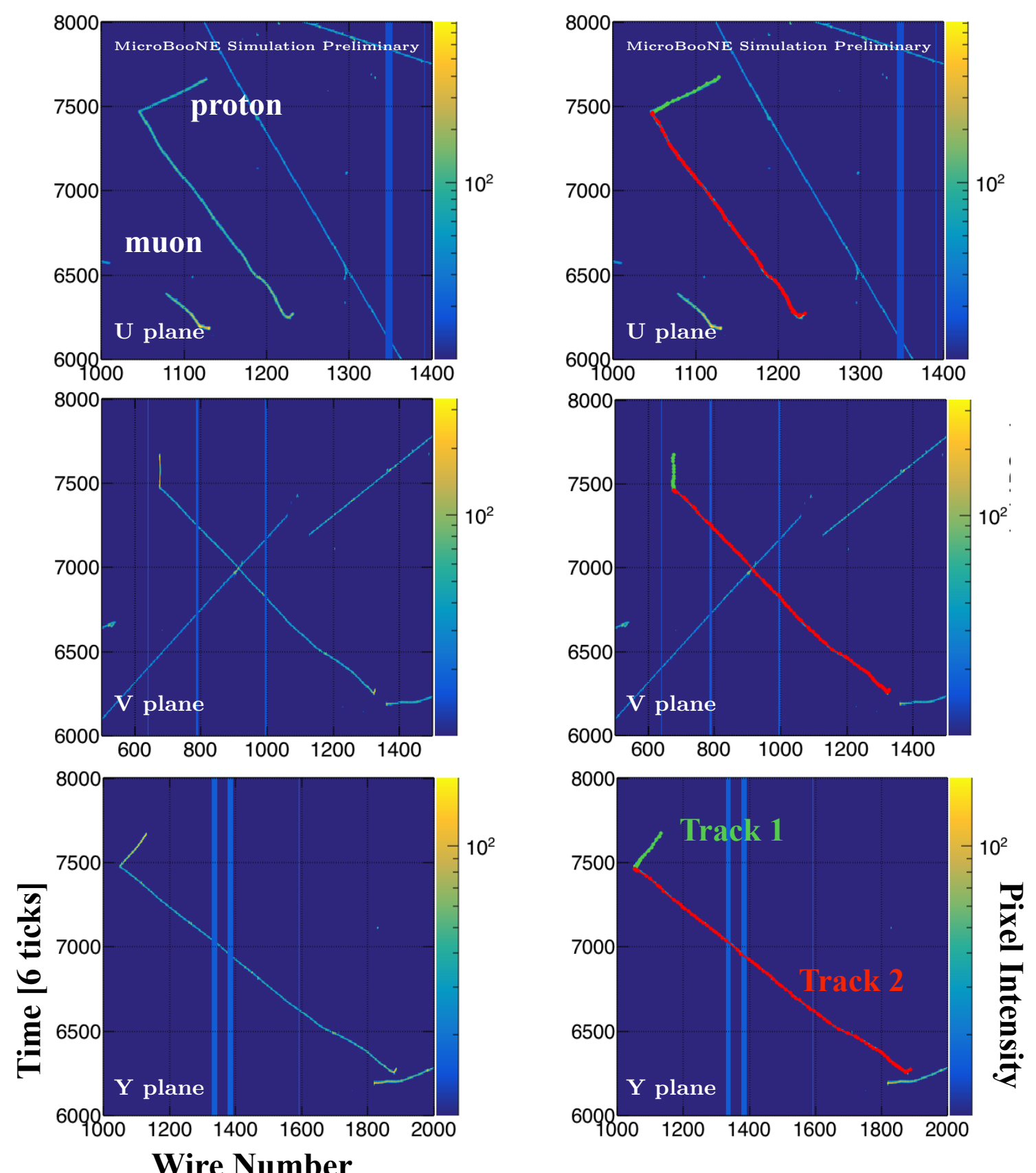

Wire Number

Figure 8.7: Tracking algorithm as applied to a $1 \mu 1 \mathrm{P}$ interaction. The left column shown the three planes event view. The right column shows the output of the tracking algorithm. Two tracks, green and red, have been reconstructed. 
detector calorimetry. Additionally, particle $d E / d x$ and kinematics can provide features for daughter particle and neutrino identification.

For $1 \mu 1 \mathrm{P}$ events the algorithm is $75 \%$ efficient at tracking both muon and proton particles together to near their true end points. An energy resolution of $\sim 3.5 \%$ is found for determining the ranged based energy of the neutrino interaction.

\section{$1 e 1 P$ Reconstruction - Section 8.8}

Reconstructing low energy electron neutrino interactions presents a challenge for generic pattern recognition algorithms developed for LArTPC experiments. As the energy of the neutrino energy decreases the topological nature of the electron and proton daughter particles changes. The proton decreases in physical length, and encodes particle ID features, such as $d E / d x$, in a smaller number of TPC wires which can challenge 3D tracking algorithms. The electron particle's features become increasingly track-like as the electromagnetic cascade decreases in size, which can challenge generic track and shower separation algorithms. The reconstruction chain after vertex finding, particle clustering, and track reconstruction is too generic to capture the features present in the low energy $1 e 1 \mathrm{P}$ neutrino topology based on background discrimination studies. An OpenCV based algorithm, called the 1e1P algorithm, is developed for reconstructing electron and proton daughter particles from $1 e 1 \mathrm{P}$ neutrino events after vertex finding.

The algorithm targets less than $600 \mathrm{MeV} 1 e 1 \mathrm{P}$ interactions and is applied in a restricted spatial region, or crop, around the candidate vertex. By working in a crop identifying the presence of cosmic rays becomes easier, as the boundary crossing points are simple to locate. In addition, candidate vertices reconstructed on background primarily exit the crop region and are vetoed. The algorithm provides dedicated pattern recognition for low energy proton and electron topological features and alleviates some reconstruction inefficiencies found earlier on in the chain. Features to identify the particle $d E / d x$ and $3 \mathrm{D}$ trajectories are reconstructed. Additionally, a match between the reconstructed particles and the in time optical flash is developed to correlate TPC activity with the optical system and provide an additional discriminate against cosmic background.

The $1 e 1 \mathrm{P}$ algorithm provides a method for determining the neutrino energy and kine- 
matics. The neutrino energy is determined using colorimetry information from the collection plane. For proton and electron particles, the energy resolution is approximately 10 and $20 \%$ respectively. The direction of the proton and electron can be determined with a resolution of 4 and $5 \%$ respectively.

\section{Particle ID Using a Convolutional Neural Network}

To characterize the neutrino interaction flavor and channel, the daughter particles must be identified. Particle identification using a deep learning technique has been shown to work effectively on LArTPC images of single particles simulated in the MicroBooNE detector as shown in [7]. In this reconstruction chain, a Convolutional Neural Network (CNN) is trained to identify five particles coming out of the vertex: $e^{-}, \gamma, \mu^{-}, \pi^{-}$, and proton using a multi-class architecture trained for an image classification task. Called the Multi-PID network, the network is trained to predict the presence of multiple types of particles in a single image. This type of network design allows for a candidate neutrino interaction, defined by the presence of vertex activity, to be used as input to the network. This scheme removes the need for individual particles to be clustered and processed through a single particle identification network, where a potential bias could be introduced due to clustering inefficiencies. The network is used to supplment traditional particle identification algorithms based on physics principles.

In practice, a cut on the network score is used to claim the presence of a particle type in the image. For $1 e 1 \mathrm{P}$ and $1 \mu 1 \mathrm{P}$ events, the network is capable of identifying the lepton particle $62 \%$ and $42 \%$ of the time respectively. The proton particle is the best classified by the Multi-PID network and is used in analysis to distinguish against neutrino and cosmic backgrounds.

\subsection{PMT Precuts}

\subsubsection{Algorithm}

The algorithm places three cuts on the optical flash, defined as a reconstructed PMT timing signal, for the event. 
1. Signal requirement

2. Maximum fractional photo-electron (PE) requirement

3. Front porch veto

The signal requirement is applied in the beam spill window defined as an optical flash at or above $20 \mathrm{PE}$. This cut eliminates a large fraction of low energy background by single photo electrons.

The fractional PE requirement requires that no single PMT contains larger than $60 \%$ of the total PE in the event.

The front porch veto eliminates events with a signal in the 130 ticks $(\sim 2 \mu \mathrm{s})$ preceding the beam spill window. The definition of a signal in the front porch is the same as in the beam spill, since cosmic activity and 1L1P events are expected to deposit similar PE in the detector. The front porch veto removes events where pre-spill cosmic ray interactions deposit significant late light or light from Michel electrons as shown in Fig. 8.8. Additionally, the veto removes events that have more than 1 flash, which can complicate the flash-charge matching that is used in the region of interest algorithm later in the reconstruction chain.

\subsubsection{Efficiency}

The 1L1P signal efficiency and cosmic ray event rejection efficiency are optimized by varying the signal PE threshold and the PMT maximum fraction cut simultaneously. CCQE events which primarily feature a 1L1P topology are used as a proxy for 1L1P events in this study. The results are shown for low energy CCQE $\nu_{\mu}$ and externally triggered BNB events in Fig. 8.9.

Good low energy neutrino efficiency is observed for a PMT maximum fraction cut of 0.5 and above and for a signal cut between 12 and $30 \mathrm{PE}$. The cut is chosen to remove at least $75 \%$ of cosmic ray only backgrounds. The effect of the PMT precuts on $\nu_{\mu}$ and $\nu_{e}$ CCQE events as a function of true neutrino energy $\left(E_{\nu}\right)$ is shown in Fig. 8.10.

For the studies described above, the software trigger was applied to both the $\nu_{\mu}$ and $\nu_{e}$ CCQE events and the BNB-EXT data events. A study was performed using an external unbiased data (EXT-UB) set to check that the software trigger does not bring in additional 


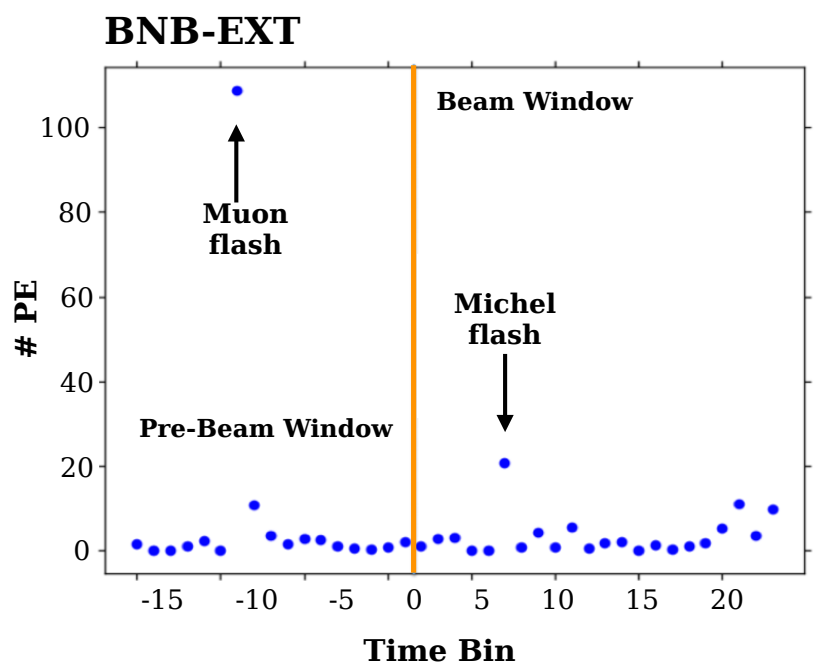

Figure 8.8: The front porch veto applied to a BNB-EXT event. The plot shows the total number of PE binned in 6 optical ticks ( $~ 90 \mathrm{~ns})$. A muon flash is identified in the pre-beam window which subsequently decays to an electron inside the beam window.

inefficiency into the PMT precut algorithm. Fig. 8.11 shows the ratio of EXT-UB with PMT precuts and software trigger to EXT-UB with PMT precuts. The result converges to $0.996 \pm 0.005$ before the signal PE threshold of $20 \mathrm{PE}$. There is negligible $(<0.5 \%)$ inefficiency due to the software trigger, because the PMT precuts impose a more stringent requirement on the number of $\mathrm{PE}$ present in the event.

In summary, the PMT precuts are $97 \%$ efficient for $\nu_{\mu}$ CCQE interactions for all $E_{\nu}$ and $96 \%$ in the 200-600 MeV range. For $\nu_{e}$ CCQE interactions the algorithm is $98 \%$ efficient for all $E_{\nu}$ and $97 \%$ in the $200-600 \mathrm{MeV}$ range. The algorithm removes $76 \%$ of cosmic ray only backgrounds. 

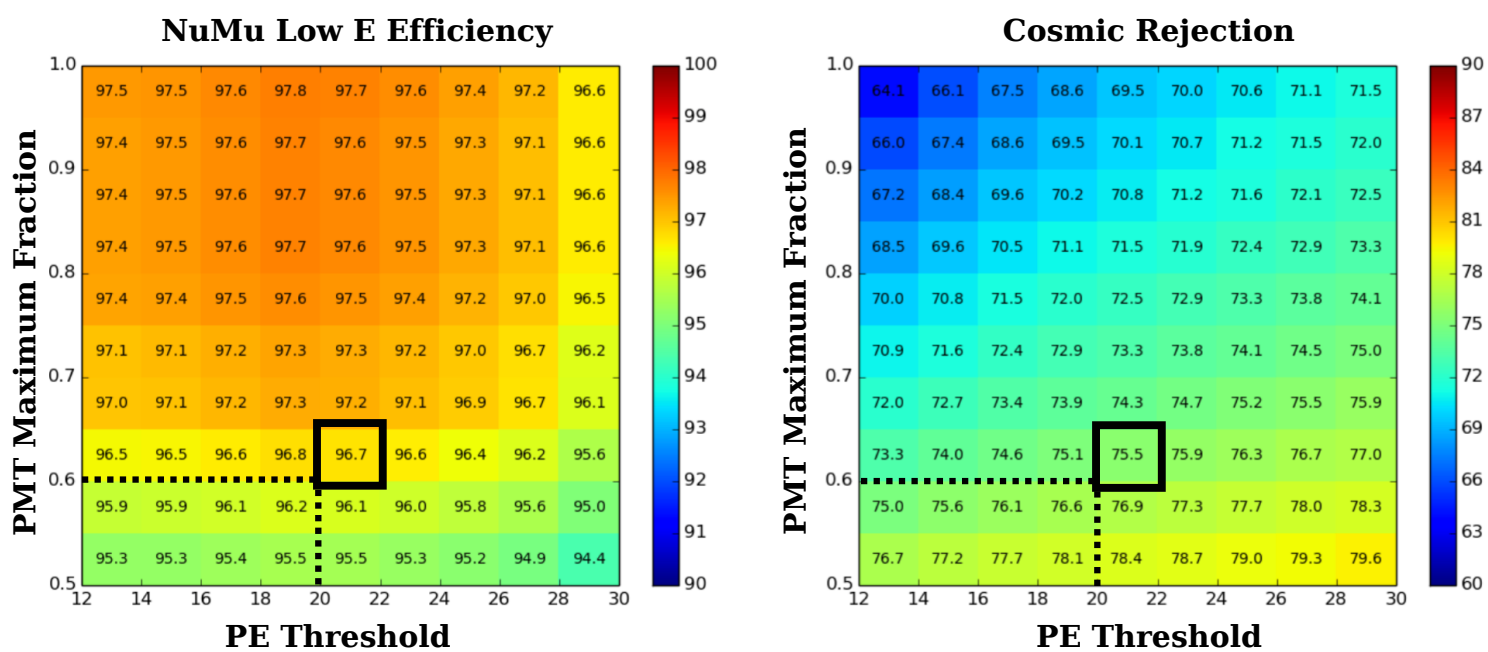

Figure 8.9: Efficiency table for $\nu_{\mu} \mathrm{CCQE}$ (left) and rejection table for cosmic ray (right) events. The efficiency is optimized as a function of PMT maximum fraction and PE threshold and are selected (black box) for the given $\nu_{\mu}$ CCQE efficiency and cosmic ray rejection efficiency.
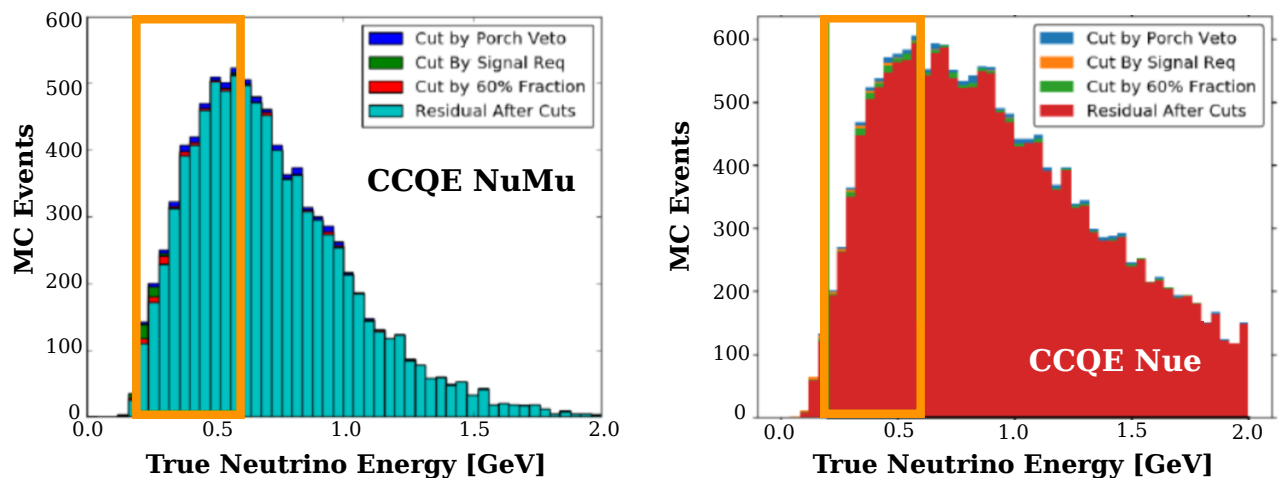

Figure 8.10: Effect of applying the PMT precuts to samples of $\nu_{\mu}$ and $\nu_{e}$ CCQE events as a function of true neutrino energy. Approximately $97 \%$ efficiency is observed in both samples with little dependence on neutrino energy. 


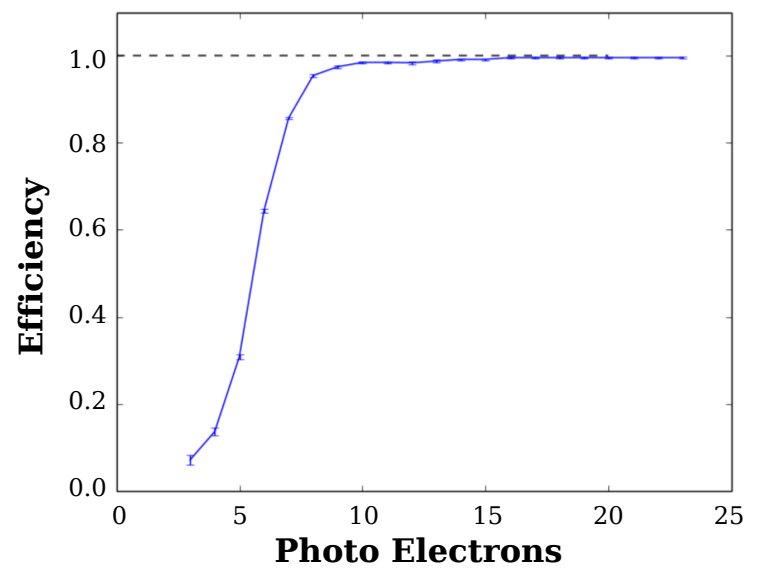

Figure 8.11: Ratio of the number of events passing both the PMT precut algorithm and the software trigger to the number of events passing the PMT precut algorithm for external unbiased data.

\subsection{Cosmic Ray Tagging \& Contained Region of Interest}

\subsubsection{Boundary Crossing Reconstruction}

To reconstruct through-going and stopping muons, points on the detector boundaries are identified. Boundary crossings occur at the top, the bottom, the upstream end, the downstream end, the cathode plane or the anode plane. Each crossing point is identified using a unique TPC or optical feature.

\section{Top or Bottom Boundaries}

The three wire planes begin or end at the top or the bottom of the detector, spanning the $\mathrm{Y}$ direction coordinate. The intersection of any three wires, one from each plane, represent uniques points at either the top or bottom. If charge is found at the location where wires meet then the particle passed through top or bottom boundary as shown in Fig. 8.12

\section{Upstream or Downstream Boundaries}

To identify particles crossing the upstream or downstream boundary of the detector the wires in each plane which are located at minimum and maximum of $\mathrm{Z}$ coordinate are used. 


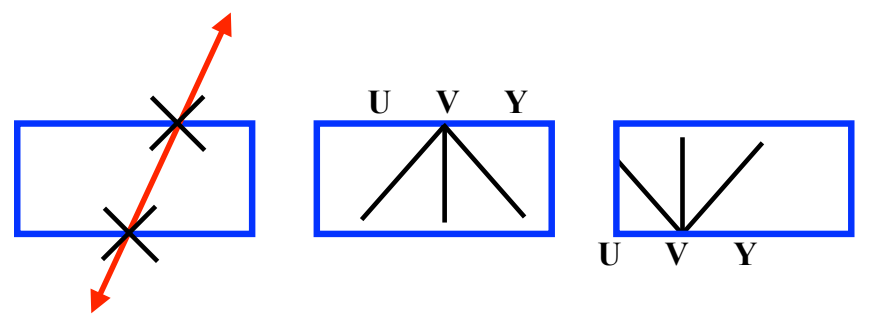

Figure 8.12: Example of a track (red line) crossing the top and bottom of the TPC. Top and bottom crossing points are identified by a coincident charge deposition on $\mathrm{U}, \mathrm{V}$, and $\mathrm{Y}$ wire plane.

As an example, a particle crossing the upstream surface of the detector will deposit charge on the first wire 0 of the collection plane $(\mathrm{Z}=0 \mathrm{~cm})$. The $\mathrm{Y}$ coordinate position of the crossing is determined by the combination of $\mathrm{U}$ and $\mathrm{V}$ plane wires that also see charge. The situation is analogous for particles crossing the downstream stream end of the detector, which will see charge on wire 3455 of the collection plane $(\mathrm{Z}=1036 \mathrm{~cm})$.

\section{Anode or Cathode Boundaries}

The scintillation light observed by the PMTs indicates the time at which a track passes through the detector. A track which crosses the anode plane produces a charge cluster where one end will be in time with the observation of light by the PMTs. For a track that crosses the cathode plane, one end of the charge cluster will appear to be at the time of a flash plus one full drift window. The anode and cathode boundaries are identified by looking for correlations between charge clusters in the image and the flashes of light by the PMTs. Fig. 8.13 shows an example of a TPC-optical correlation for an anode crossing muon.

The procedure for finding boundary crossing points is straightforward with perfect detector operation. In practice, two challenges introduce inefficiencies in boundary point reconstruction. First, approximately $10 \%$ of the wires in the detector are unresponsive or produce an unreliable signal. For some portion of the detector boundary, it is not enough to search for charge on all three wires. In this case, the algorithm looks for charge on two of the three wire planes and the presence of a bad channel tag for the corresponding wire on 


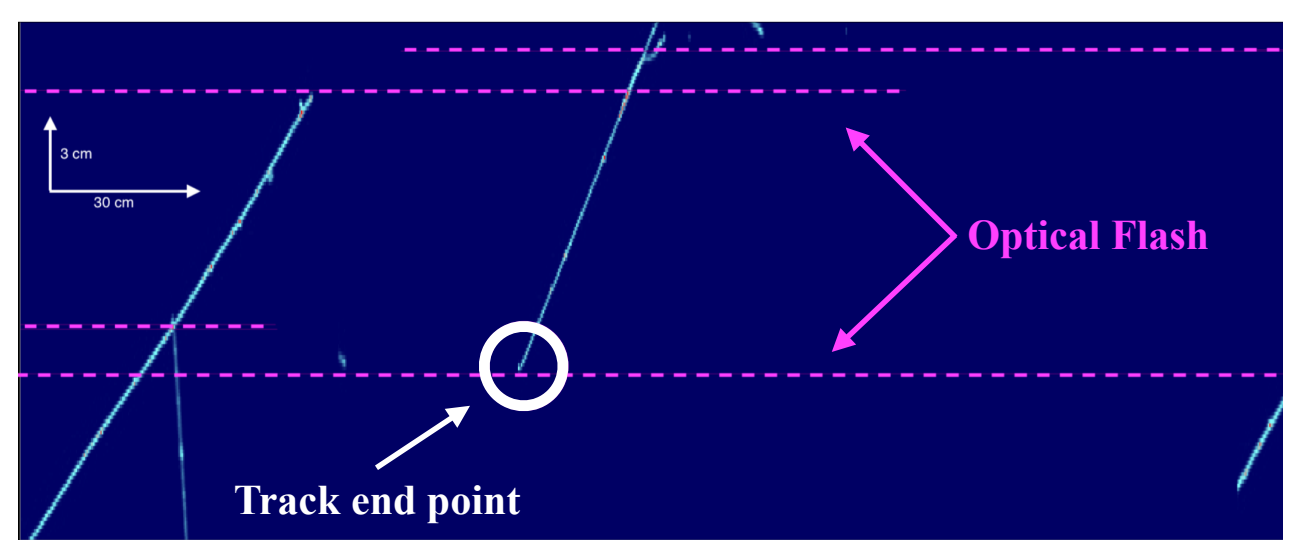

Figure 8.13: An image of the Y-plane near the end of a stopping muon particle. The lowest purple line indicates the time of a cluster of PMT hits, called an optical flash. The upper line is the time of another optical flash plus the a single detector drift time. The track stops on the lower line and crosses the upper line. This track is consistent with entering or exiting the anode plane but not the cathode plane.

the remaining wire plane. Secondly, space charge effects [32] will distort where the charge produced at the boundary will appear to be measured. Due to convection currents in the liquid argon bulk, we expect that positive argon ions, produced by charged particle tracks ionizing the detector medium, to concentrate near the middle of the detector. These ions distort the electric field created by the cathode and anode. Positive charge builds up in the center of the detector, causing the ionization electrons to drift towards the detector center in addition to traveling towards the anode wire plane. As a result, the ends of the charge clusters left by the cosmic track will appear some distance away from their true position inside the active volume of the detector. The magnitude of the space charge effect varies as a function of the $\mathrm{X}$ detector coordinate. The algorithm which finds the boundary crossing points takes into account unresponsive wires and space charge effects by carrying an error tolerance.

The end result of the boundary crossing point algorithms is a list of candidate $(x, y, z)$ points. The metric used to check the boundary crossing algorithm is by counting the fraction of MC cosmic muon crossing points that are tagged. Crossing muon points were found by using the truth information to check the start and endpoints of cosmic muons. If a track 
end was less than $10 \mathrm{~cm}$ from active volume boundary the track end is considered a true crossing point. To be considered tagged, at least one reconstructed boundary crossing point needs to be identified within $10 \mathrm{~cm}$ of the true crossing point. Fig. 8.14 shows the fraction of true crossing points that are tagged by the algorithm.
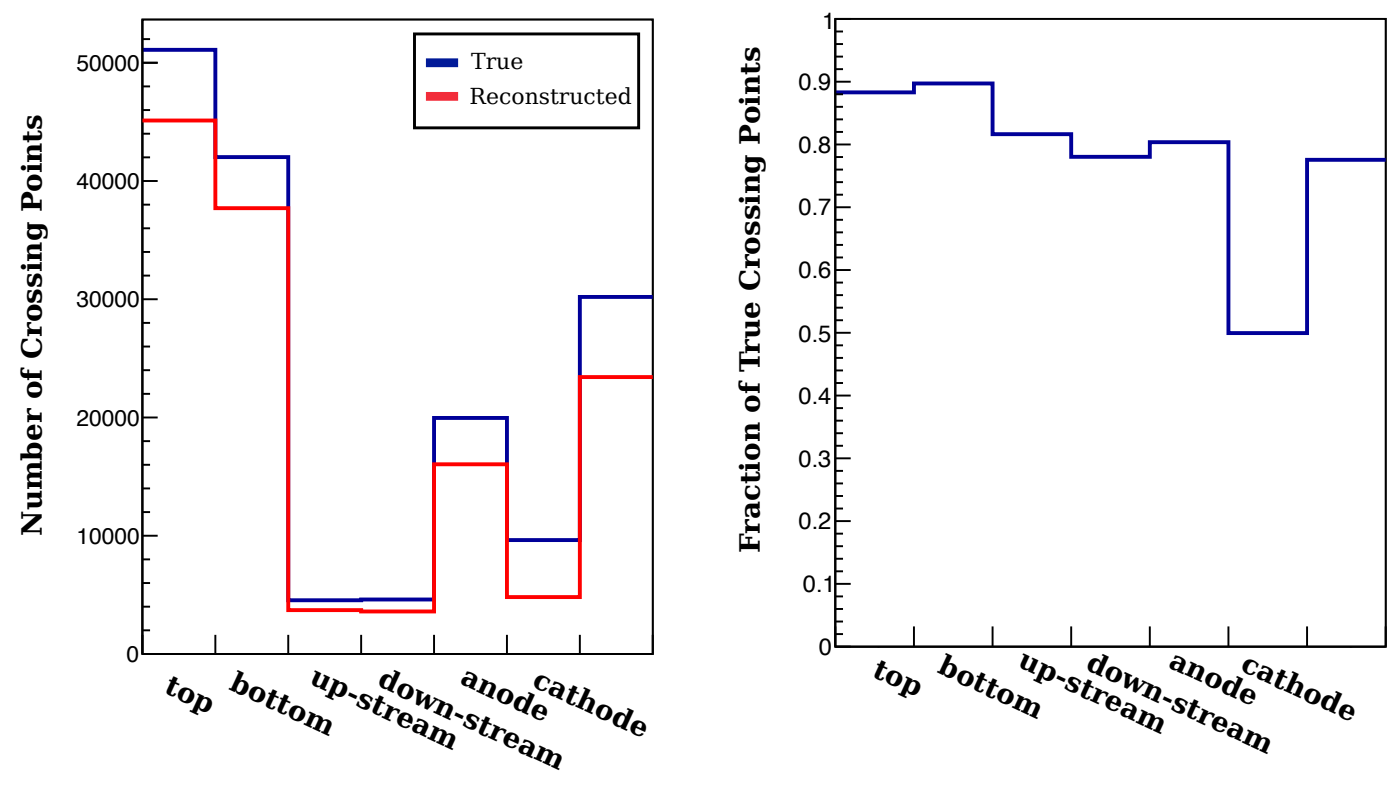

Figure 8.14: Performance of the boundary point tagging algorithm for six types of detector boundaries. Left: Number of true (blue), and reconstructed (red) crossing points for each boundary type. Right: Ratio of the number of reconstructed to true crossing points. Top and bottom points are best reconstructed correctly while approximately half of anode crossing points are reconstructed.

On average, $85 \%$ of the track ends are found by the boundary crossing tagger. This fraction remains flat as the complexity of the image, measured by the number of occupied pixels, increases as demonstrated in Fig. 8.15

\subsubsection{Through-Going Muon Reconstruction}

Through-going particles are muons which feature a straight track with slight curvature, due to the space charge effects, connected at two boundary crossings. An algorithm, called ThruMu, reconstructs through-going muon particles. 

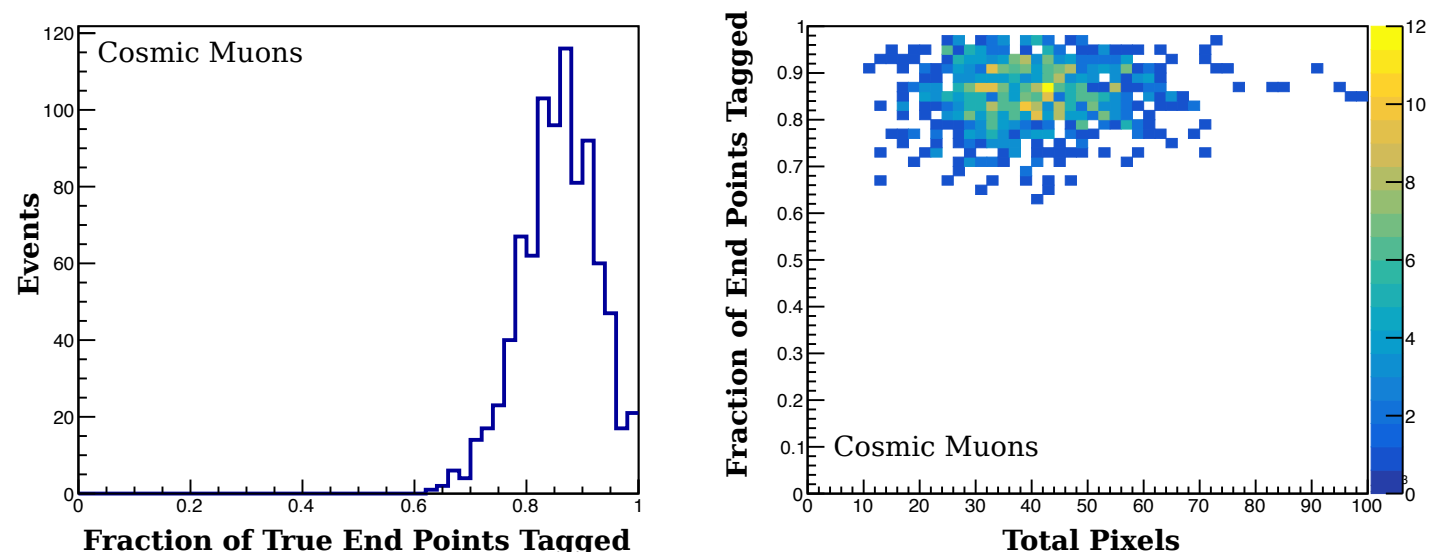

Figure 8.15: Left: Fraction of true crossing muon end points tagged by the ThruMu tagger. On average, an MC event will have approximately $80 \%$ or more end points identified correctly. Right: The fraction of end points tagged versus the total number of pixels in the image.

The algorithm begins by selecting a pair of boundary crossings and executes a path finding algorithm to follow charge between the pair of points. Two path finding algorithms are used based on track topology. First, a straight line is drawn in 3D between the boundary points and projected into each of the three views separately. A search is performed in each view of pixels lying along the straight line, within a distance of five pixels, stepping forward in the drift direction. At each point in time, all three views must find charge; or else two views must find charge, while the third has a tagged bad region. This allows the algorithm to proceed through detector regions where one plane is dead. If $95 \%$ of the steps along the track have charge where expected then all pixels found in the search are tagged as throughgoing. The other $5 \%$ of pixels that did not satisfy the charge and bad channel requirement are also included in the ThruMu track and are tagged as ThruMu. If the amount of observed charge is between $20 \%$ and $95 \%$ of what is expected, then a second algorithm based on the A* path-finding method [33, which looks for tracks with non-linear deviations is applied. In the $\mathrm{A}^{*}$ path-finding algorithm, the images are compressed such that each pixel becomes 4 wires by 24 time ticks $(1.2 \mathrm{~cm}$ in wire view and $1.3 \mathrm{~cm}$ in time view). This allows for faster search over the image by reducing the resolution of the $3 \mathrm{D}$ grid. A single bad and dead 
pixel, therefore, now has 4 wires that are failing to respond. If a path can be formed, then a ThruMu candidate is created. Finally, the image is decompressed and the pixels along the path are tagged as ThruMu. An example of ThruMu tracking is shown in Fig. 8.16 for a simulated event.

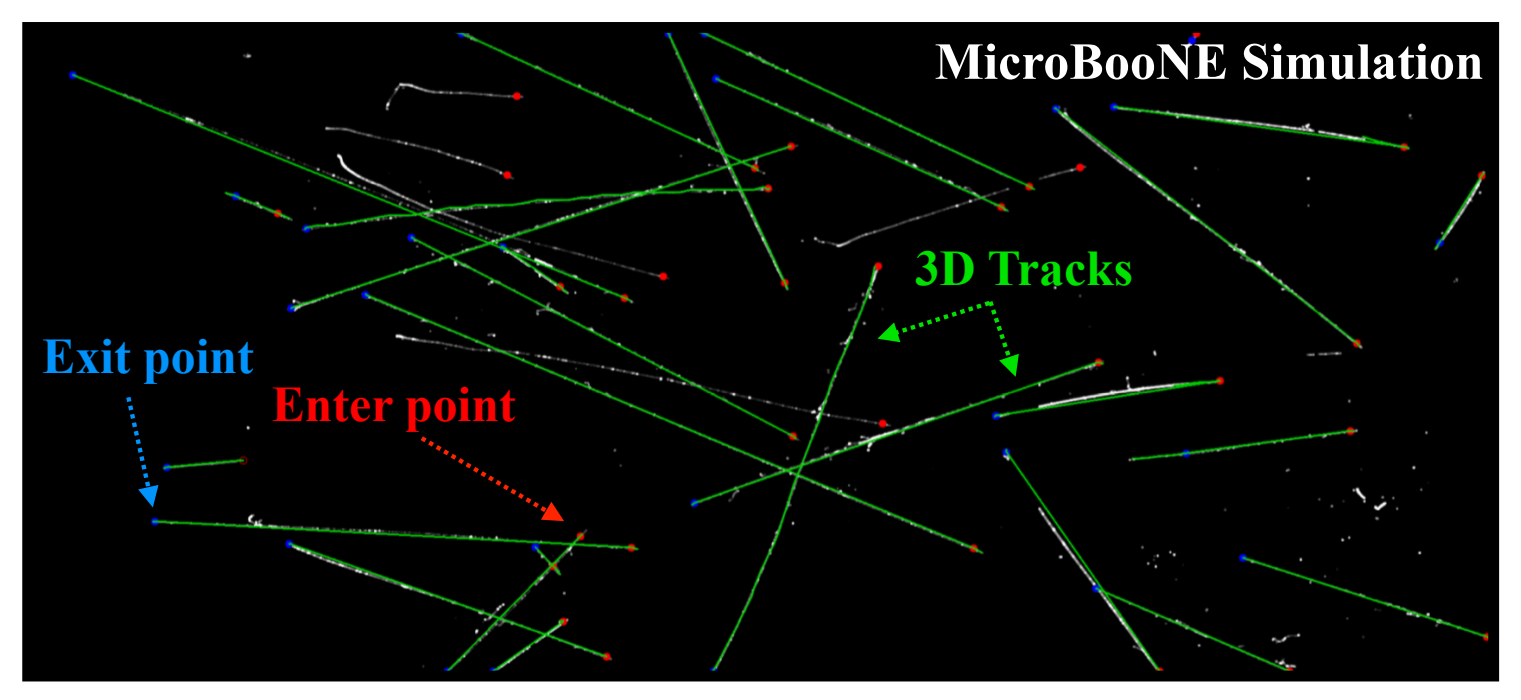

Figure 8.16: Example of ThruMu tracking applied to a simulated cosmic ray event. Crossing muon entry (red) and exit (blue) points are shown. Green lines represent the output of the ThruMu algorithm.

\subsubsection{Stopping Muon Reconstruction}

After ThruMu tracks are reconstructed, the remaining pairs of boundary points along with the set of untagged pixels are scanned using an algorithm which identifies stopping muon particles, called StopMu. The StopMu algorithm begins by taking steps away from a boundary point of a defined length that satisfies two constraints. First, the path covered by the step must project back onto all three planes with charge. Second, if multiple steps are possible the track will preferentially follow the previously found direction. By iterating using these criterion, the algorithm builds a 3D path for the candidate stopping muon into the detector. An example of stopping muons found by the StopMu algorithm is shown in Fig. 8.17. 


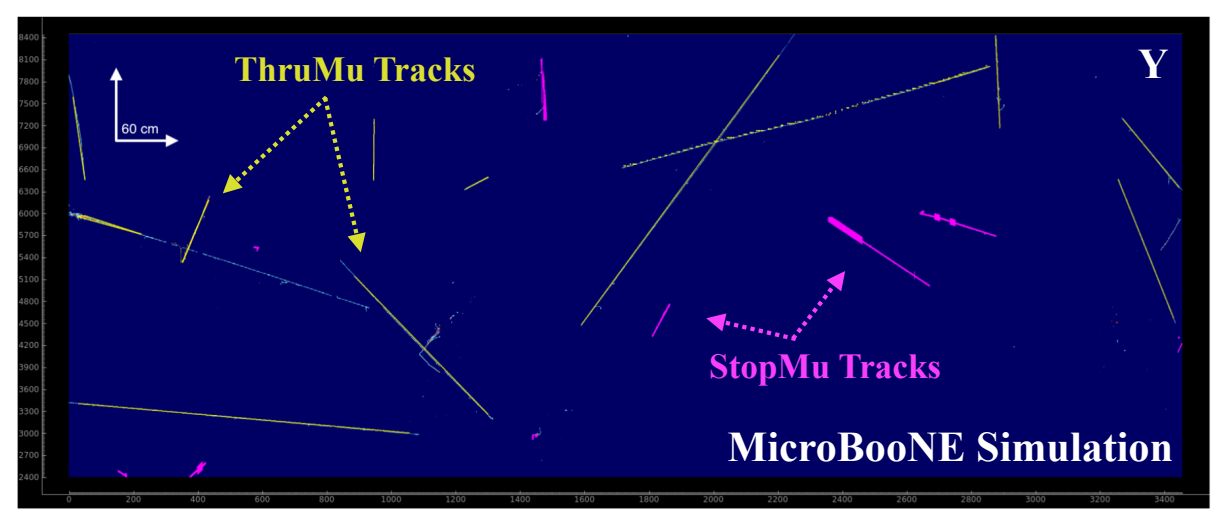

Figure 8.17: An example MC intrinsic $\nu_{e}$ events with CORSIKA cosmic rays. ThruMu and StopMu tracks are shown as yellow and pink lines respectively.

\subsubsection{Cosmic Pixel Tagging Efficiency}

The cosmic tagging algorithms are analyzed by studying the fraction of pixels on all three planes above a threshold that are tagged. Fig. 8.18 shows the fraction of pixels with an intensity value above 10 that have been tagged by the ThruMu, ThruMu and StopMu, and pixels which remain un tagged. On average the ThruMu and StopMu algorithms tag approximately $75 \%$ of all above threshold pixels in an image. After the StopMu algorithm is applied, the pixel fraction does not change significantly. In the reconstruction and analysis chain the StopMu algorithm is disabled due to poor performance on simulated data.

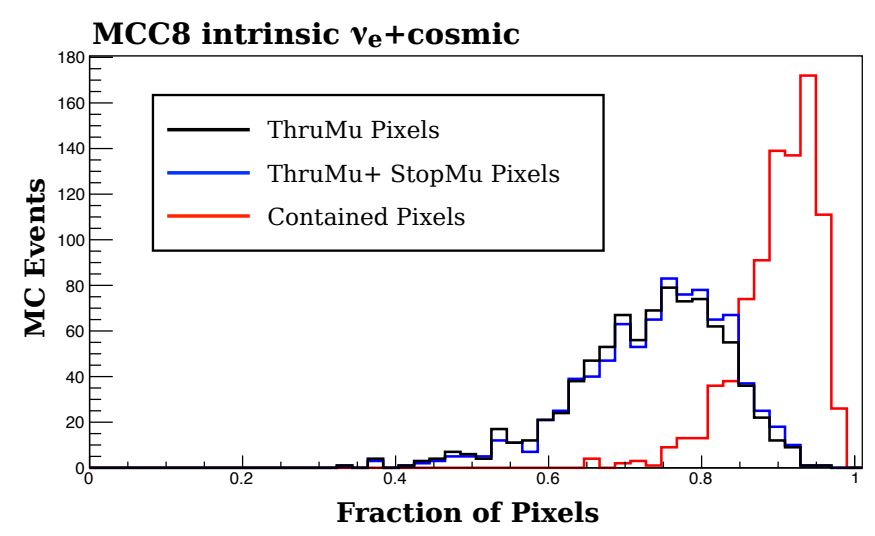

Figure 8.18: Distribution of cosmic tagged pixels for a sample of MCC8 intrinsic $\nu_{e}$ with CORSIKA cosmic rays. 
The performance of the ThruMu algorithm is profiled on CORSIKA cosmic muon MC. To separate issues of the ThruMu tracker from those of the boundary crossing point finder, truth information is used for the start and end points of the muons to the algorithm. Given true boundary point locations, the algorithm can successfully tag over $90 \%$ of the tracks from start point to end point.

One point of inefficiency introduced by the cosmic ray tagger is the misidentification of neutrino induced pixels as cosmic rays. This can occur via two primary means. First, if the neutrino interaction interacts inside the TPC and is large enough in energy, the daughter particles may exit the TPC, producing a boundary point. This boundary point may be connected to another boundary point via the tracking algorithm and cause neutrino daughter particles to be tagged as cosmic ray. The second way is if a through-going cosmic ray crosses contained neutrino charge in the plane projection. When this occurs, the cosmic tagger can follow the cosmic track onto the neutrino pixels and partially label them as cosmic ray. An example is shown in the top of Fig. 8.19.

Using a sample of $1 e 1 \mathrm{P}$ events, approximately $50 \%$ of interactions have at least $10 \%$ of their visible neutrino pixels tagged as cosmic ray. The interference of the neutrino interaction by the cosmic tagger is partially alleviated by detailed topological reconstruction described in Section 8.8.

\subsubsection{Contained Region of Interest Reconstruction}

The optical flash information of the event contains an indication of neutrino activity present inside the TPC. An algorithm is designed to localize a $\mathrm{Z}$ region of the detector where the in time flash occurred, called a Contained Region of Interest, or cROI. An example of two cROI on top a LArCV event image is shown in Fig. 8.20.

The cROI is of fixed size and placed on the event image at the mean $\mathrm{Z}$ position of the flash. The mean flash position is defined as the PE weighted PMT position. The cROI defines a 3D region in TPC space to execute detailed reconstruction of neutrino activity.

The cROI efficiency is shown in Fig. 8.21 and is defined as the ratio of events for which the true neutrino vertex is located inside the cROI to all simulated events.

The two plane cROI efficiency is approximately $95 \%$ or larger with minimal dependence 

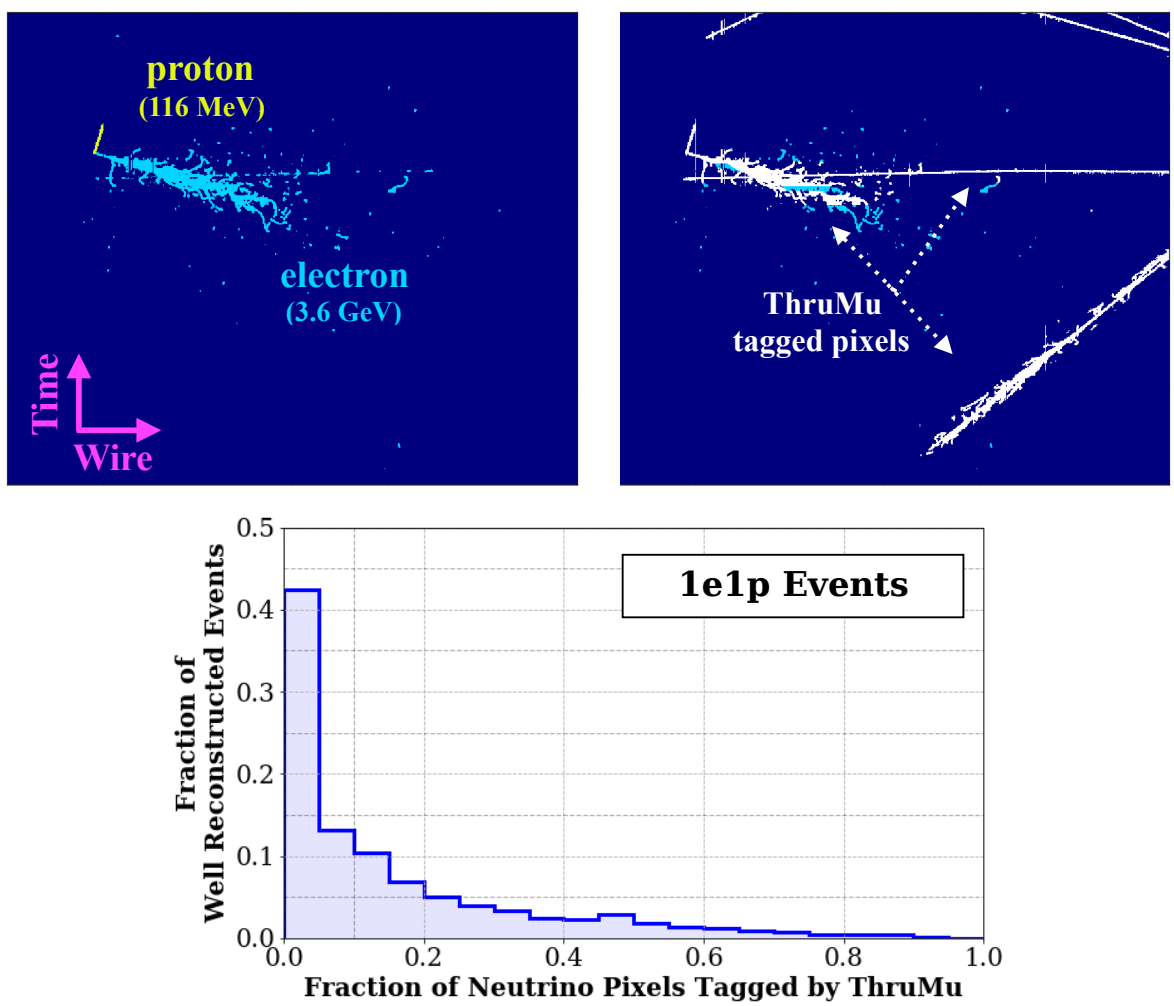

Figure 8.19: Top: Example of a 1e1P interaction on the Y plane in false color scale with cosmic rays artificially removed. The right image shows the effect of the cosmic tagging algorithm (white pixels) applied to the event. Bottom: The distribution of fraction of neutrino pixels tagged as ThruMu for a sample of well reconstructed $1 e 1 \mathrm{p}$ events.

on the true neutrino energy.

\subsection{Track and Shower Pixel Identification using Semantic Segmentation}

\subsubsection{Network Architecture}

The SSNet (Semantic Segmentation Network) architecture used is called U-ResNet which is a hybrid of the U-Net [34], which has been used to identify pixels in microscopic images of living cells for biomedical research, and ResNet [35]. An overview of the U-ResNet architecture is shown in Fig. 8.22 . 


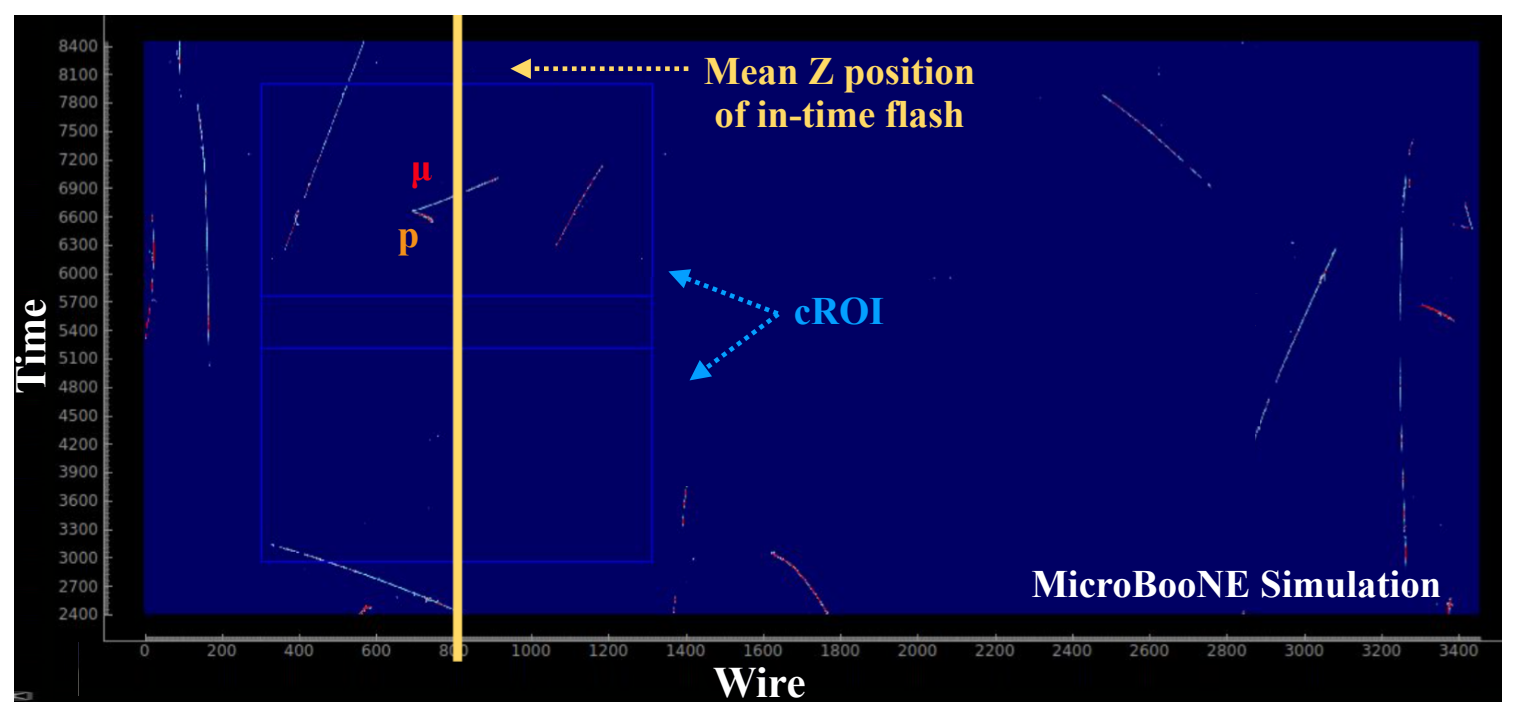

Figure 8.20: Example 1 $\mu 1 \mathrm{P}$ collection plane event image featuring two cROI (blue boxes) placed at the mean position of the in-time flash (beige line). The cROI defines a sub region of the detector space for further pattern recognition.

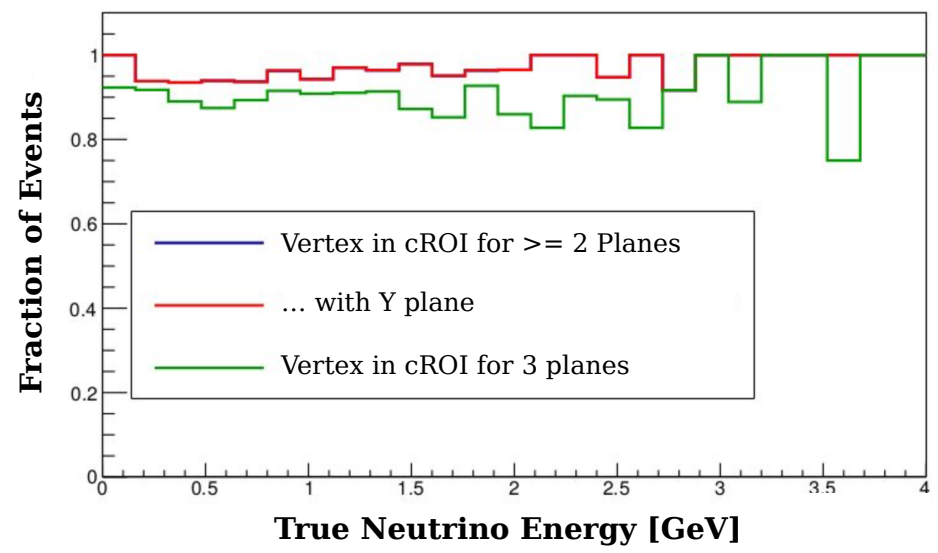

Figure 8.21: Efficiency of placing cROI on the event image consistent with the true neutrino vertex location as a function of true neutrino energy. The blue and red curves show the efficiency for placing the cROI on the image covering the neutrino vertex in at least two planes, and requiring one of them to be the collection plane respectively. The green curve shows the efficiency for placing the cROI on the image covering the vertex in all three TPC planes. 


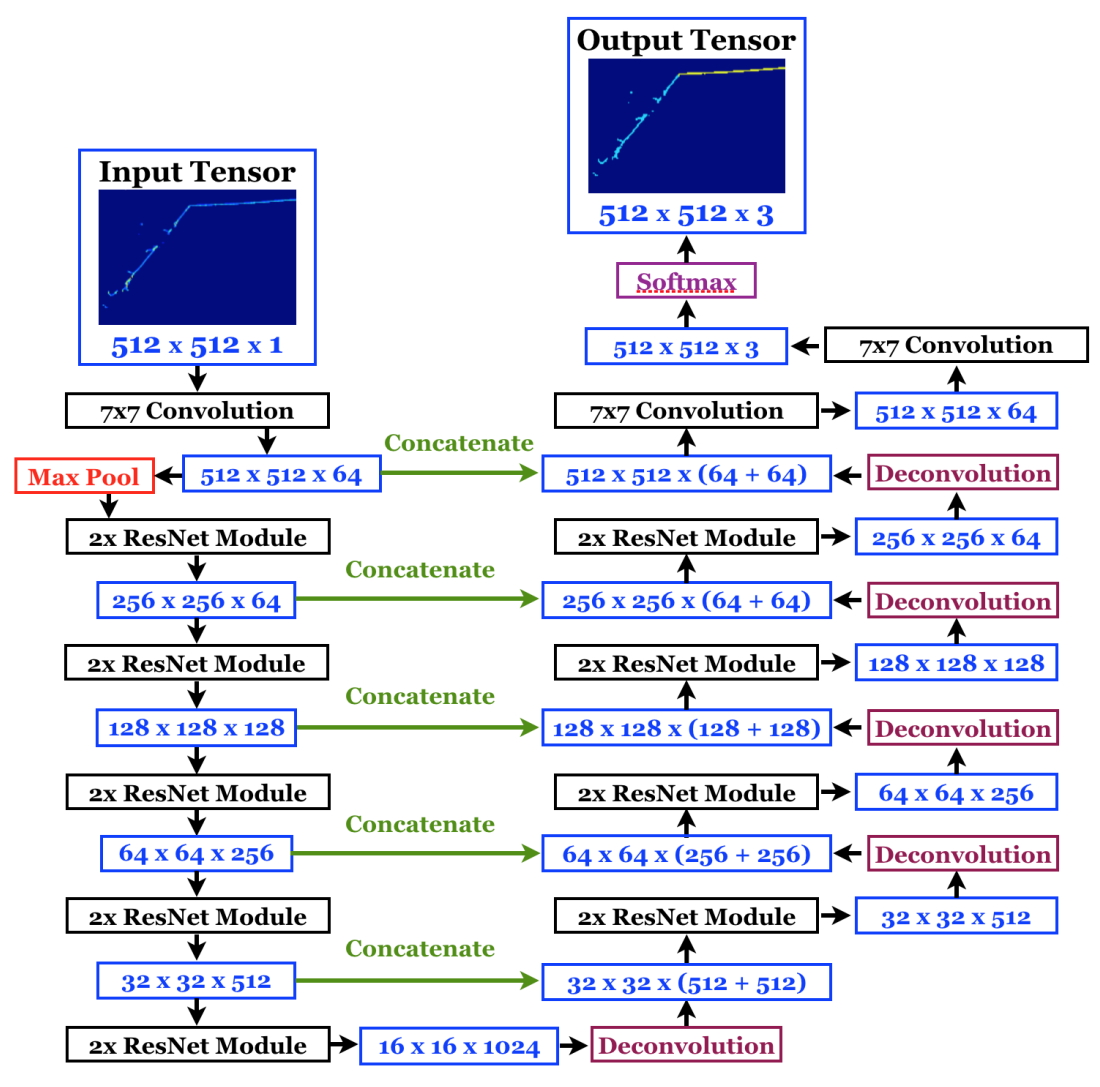

Figure 8.22: Diagram of U-ResNet architecture. A 512 x 512 single channel image is input. Black arrows describe the direction of tensor data flow. Dashed green lines indicate concatenation operations to combine filter outputs from the convolution layers to the input of deconvolution layers. The final output has the same spatial dimension as the input except for the depth which becomes 3 channels, one per pixel class

The structure of U-ResNet follows a typical network design for a SSNet. The architecture consists of two parts. The first part of the network mimics that of an image classification CNN and is responsible for learning complex image features. The left-half of Fig. 8.22 corresponds to this first component, sometimes referred to as the convolutional half, and can be seen as encoding the image onto different image features. This half of U-ResNet contracts the spatial dimension from the input size of $512 \times 512$ pixels down to $16 \times 16$ pixels through 11 convolution blocks where the number of feature maps becomes 1024 .

Starting from the output of the first half, the second half of a SSNet expands the spatial 
dimension by successive interpolation or up-sampling layers. In other words the second-half of the network, referred sometimes as the deconvolution half, projects the encoded feature information back into image. Here, deconvolution refers to backward strided convolution or interpolation. Using the projected image features, the last layers of the network's second half makes a classification prediction for each pixel. The right half of U-ResNet performs expansion of the spatial dimension from $16 \times 16$ to $512 \times 512$ by a series of deconvolution operations.

The output of U-ResNet is an image with the same spatial dimension as the original input but expanded to 3 channels. Each channel contains a pixel-wise score of being one of three types: background, shower, or track. Fig. 8.23 shows a visualization of the network output on the right column for electron and proton final state from a $1 e 1 \mathrm{P}$ interaction.
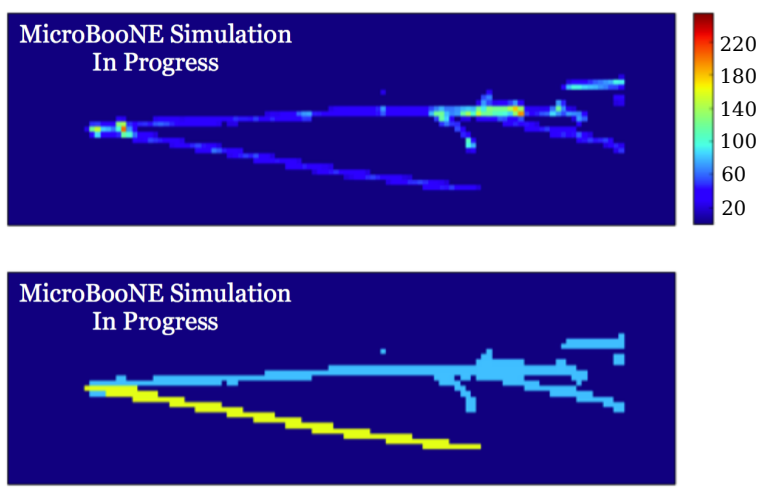

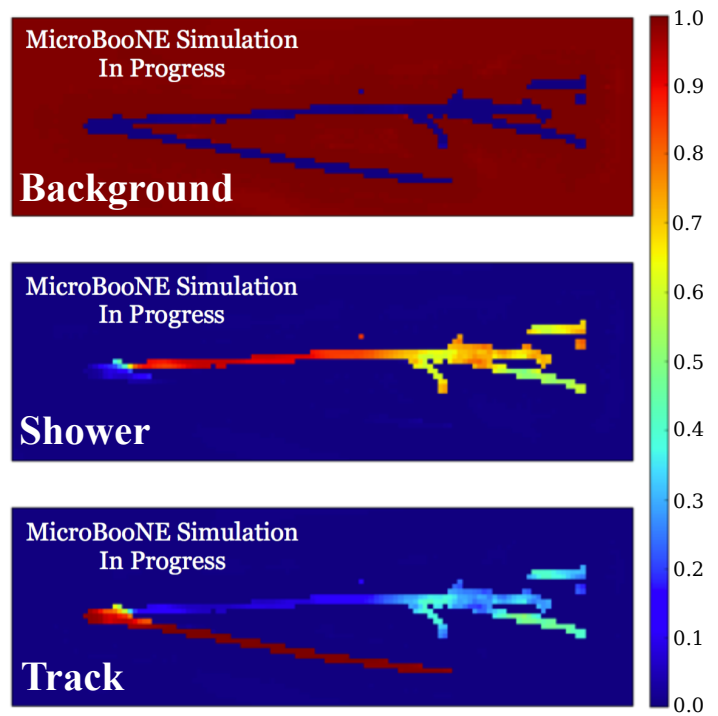

Figure 8.23: Visualization of example input and output data tensors for a $1 e 1 \mathrm{P}$ interaction. The left column shows the input LArCV image (top) and the true pixel-wise label (bottom) used to train the SSNet. The labeled image shows track pixels in yellow, and shower pixels in cyan. The right column shows the network output which is a pixel-wise normalized score of being a background (top), shower (middle), and track (bottom) type.

The class label per pixel is determined by taking the label with the largest score at each pixel point. 


\subsubsection{Network Training}

The U-ResNet architecture is implemented using Caffe [36] and customized [37] to employ spatial loss weighting. A transfer learning technique is employed by first training the firsthalf of U-ResNet for an image classification task using the identical data set from described in [7]. The data set contains single particle images of electron, photon, muon, pion, or proton. The network's weights, trained first to discriminate between different particle images, provide a initial state to perform pixel-level track and shower separation.

Both sides of U-ResNet network are trained starting with pre-trained weights for the convolution side as described above, and randomly initialized weights for the deconvolution side. Training data is produced using a custom event generator called MultiPartVertex $(\mathrm{MPV})$. The generator produces simulated 3D interaction vertices at random locations inside the MicroBooNE TPC with no neutrino model dependence. Five types of particles are simulated: electron, gamma, muon, pion, and proton. The particles are emitted with isotropic momentum from the vertex. The data set consists of 140,000 images and contains the following particle and multiplicity distributions:

- $80 \%$ of events are simulated requiring one of the generated particles to be a electron or muon with kinetic energy ranging from 50 to $1000 \mathrm{MeV}$. For the other generated particles, the MPV is configured to randomly assign their types to a photon, charged pion, proton, or another lepton.

- $20 \%$ of events are generated with random particle type and the total multiplicity is set randomly between one and four particles. For each particle type, the maximum multiplicity is set to two. The ranges for the randomly assigned momentum are specified as 30 to $100 \mathrm{MeV} / \mathrm{c}$ for electron and photon, 85 to $175 \mathrm{MeV} / \mathrm{c}$ for muon, 95 to $195 \mathrm{MeV} / \mathrm{c}$ for pion, and 300 to $450 \mathrm{MeV} / \mathrm{c}$ for proton.

Additionally, a pixel wise loss is implemented to focus the network's attention on nonbackground pixels in the image as shown in shown in Fig. 8.24. The first category contains background (i.e., zero) pixels that surround non-background (i.e., non-zero) pixels within 2 pixels. The second category is the rest of background pixels in the image that do not belong to the first category. The third category represents non-zero pixels within 4 pixels of 
the generated event vertex. Finally, the fourth category is defined for each particle instance and includes non-zero pixels that belong to a particle.
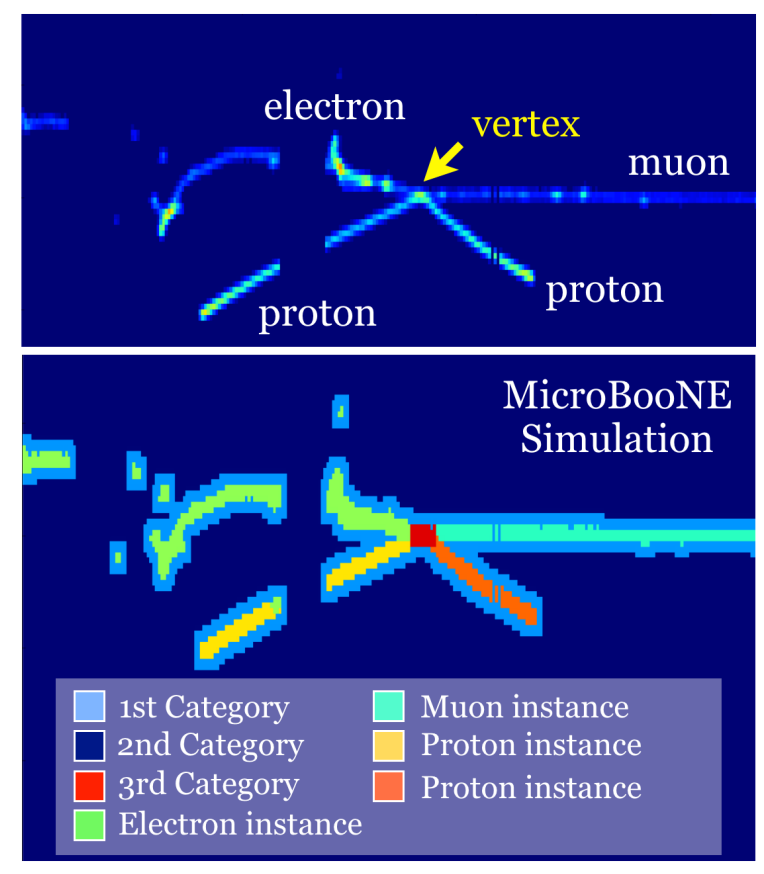

Figure 8.24: Top: An example LArCV image from the training set in which two protons, one electron, and one muon are produced. Bottom: The event from the top image that shows the categories for pixel-wire loss weighting indicated in different colors.

The RMSProp algorithm [38] is used for optimization with a batch size of 50 images and performed on a NVIDIA Titan X GPU. The loss and accuracy, defined as Incorrectly Classified Pixel Fraction (ICPF), as a function of epoch is shown in Fig. 8.25.

\subsubsection{Performance}

The performance of U-ResNet is benchmarked on test simulation samples of $1 \mathrm{e} 1 \mathrm{P}$ events, and low energy $1 \mu 1 \mathrm{P}$ and $1 e 1 \mathrm{P}$ events. All samples are generated using the MPV generator as described in Section 8.5.2. The samples are configured to generate one proton and one lepton only. Particles are simulated with a uniform energy distribution and isotropic momentum direction distribution. The kinetic energy range is set to be 50 to $500 \mathrm{MeV}$ for electron and 50 to $300 \mathrm{MeV}$ for protons. In addition, there are two low energy MPV samples 
(a)

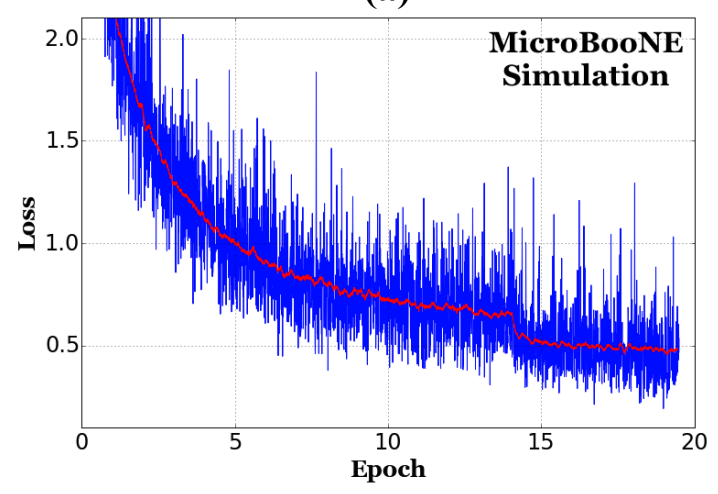

(b)

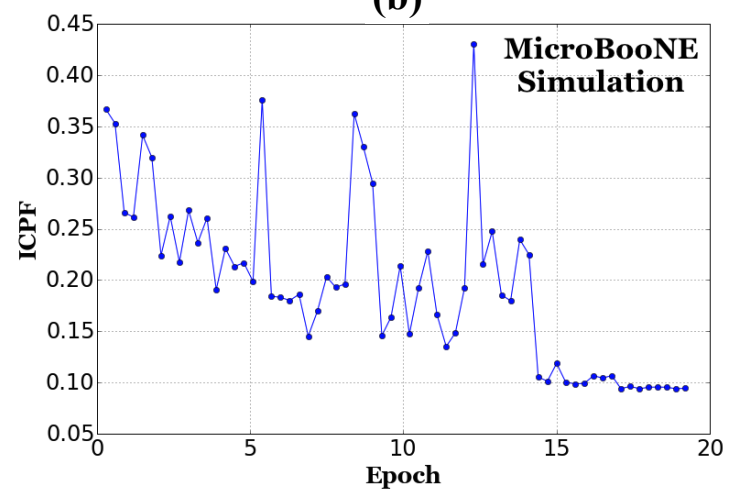

Figure 8.25: (a) The loss value as a function of training time using the validation sample. The red line shows the average at a given epoch using 200 the neighboring points. (b) Incorrectly Classified Pixel Fraction (ICPF) as a function of training time using the validation sample. The drop in both plots at epoch 14 is due to lowering of the learning rate by a factor of 10 .

generated: low energy $1 e 1 \mathrm{P}(1 e 1 \mathrm{P}-\mathrm{LE})$ and low energy $1 \mu 1 \mathrm{P}(1 \mu 1 \mathrm{P}-\mathrm{LE})$. For $1 e 1 \mathrm{P}-\mathrm{LE}$, the electron has momentum distributed from 30 to $100 \mathrm{MeV} / \mathrm{c}$. For $1 \mu 1 \mathrm{p}-\mathrm{LE}$, the momentum of muon is distributed from 85 to $175 \mathrm{MeV} / \mathrm{c}$. In both samples, the momentum of the proton is distributed from 300 to $450 \mathrm{MeV} / \mathrm{c}$. Four metrics based on the error rate are used. The error rate is defined as,

$$
\text { Error rate }=1-\frac{1}{N} \sum_{\text {image }}^{N}\left(\frac{\text { Number of correctly labelled non }- \text { zero pixels }}{\text { Number of total non }- \text { zero pixels }}\right)
$$

- ICPF mean: the average value of incorrectly classified pixel fraction per image computed over all events in a sample. The ICPF metric is the combined track and shower pixel label error rate.

- ICPF $90 \%$ quantile: the ICPF value below which $90 \%$ of events in a sample are present.

- Shower error rate: the average value of the shower pixel only error rate, averaged over all images in a sample.

- Track error rate: the average value of the track pixel only error rate, averaged over 
all images in a sample.

The results can be found in Table 8.1 .

\begin{tabular}{|c|c|c|c|c|}
\hline Sample & ICPF Mean [\%] & ICPF 90\% [\%] & Shower [\%] & Track [\%] \\
\hline $1 e 1 \mathrm{P}$ & 2.2 & 5.7 & 2.8 & 4.0 \\
\hline $1 e 1 \mathrm{P}-\mathrm{LE}$ & 3.9 & 11.5 & 3.8 & 8.0 \\
\hline $1 \mu 1 \mathrm{P}-\mathrm{LE}$ & 2.3 & 2.2 & 6.2 & 2.4 \\
\hline
\end{tabular}

Table 8.1: Values of the four metrics defined in Section 8.5.3 to benchmark the SSNet performance.

The network is generalized to perform well on simulated neutrino events to a level that allows us to apply the technique as a part of the reconstruction chain. We do not train the SSNet on our 1L1P signal predictions as simulated by GENIE because this may introduce a model bias. The benchmark results on 1L1P-LE events demonstrate that the U-ResNet can classify pixels from the low energy two particle topologies of track and shower at the ICPF mean value of $3.9 \%$ and $2.3 \%$, respectively. In the $1 \mu 1 \mathrm{p}-\mathrm{LE}$ sample, despite the fact that no showers are produced in the primary neutrino interaction, challenges for the network arise from similarities between muons and electrons at very low energies and from secondary interactions like Michel electrons from muon decays. The error rate for the $1 e 1 \mathrm{P}$ and 1e1P-LE samples as a function of kinematic variables is shown in Fig. 8.26.

Fig. 8.26(c) shows the correlation with the opening angle between the two particles in 1e1p sample. The error value is expected to increase when the two particles are colinear and the $2 \mathrm{D}$ projections of the particles overlap. When the particles are back-to-back, the difficulty in distinguishing them arises from the fact that two trajectories may appear as the trajectory of one particle. Fig. 8.26(a) and (b) show the dependence of the performance on the kinetic energy of a particle from 1e1P-LE sample. The network performs worse at lower energies. The ICPF value reaches approximately $15 \%$ at $50 \mathrm{MeV}$ proton kinetic energy. A proton at this energy only travels a few centimeters in liquid argon, which translates into 10 pixels or less in the collection plane image. Such a small amount of information makes the networks task difficult. A similar trend of decreasing performance can be also seen for electron kinetic energy. The critical energy above which electrons primarily produce 
(a)
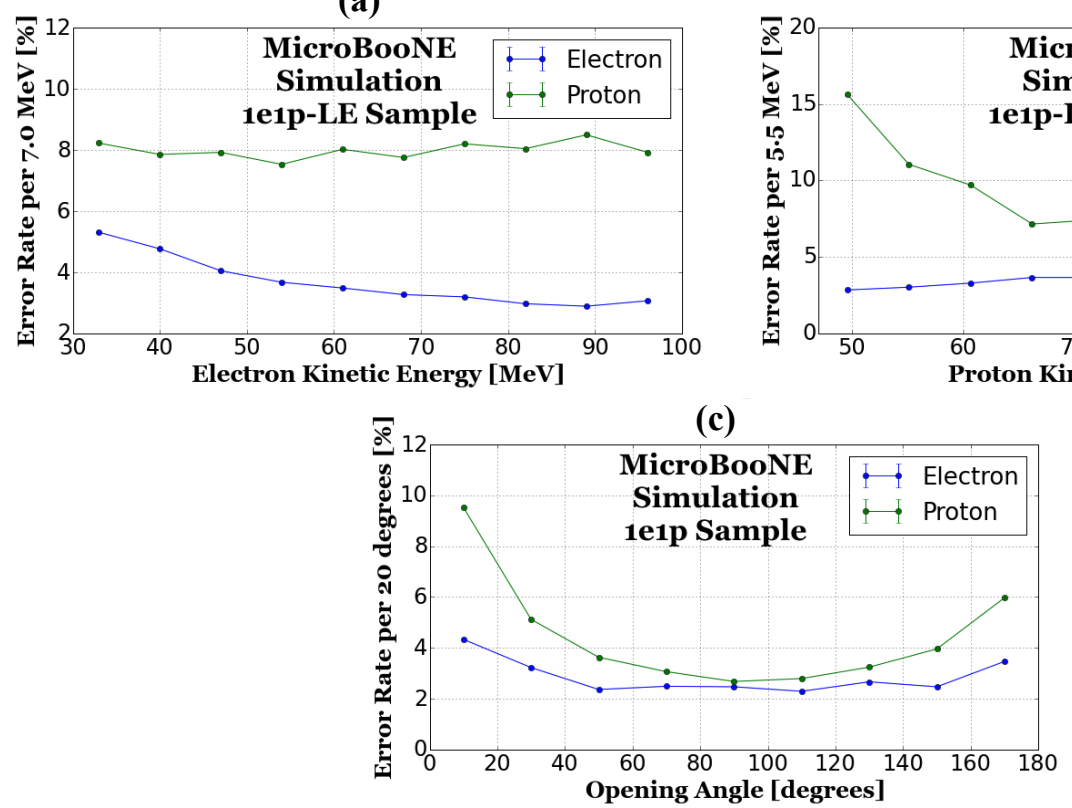

(b)

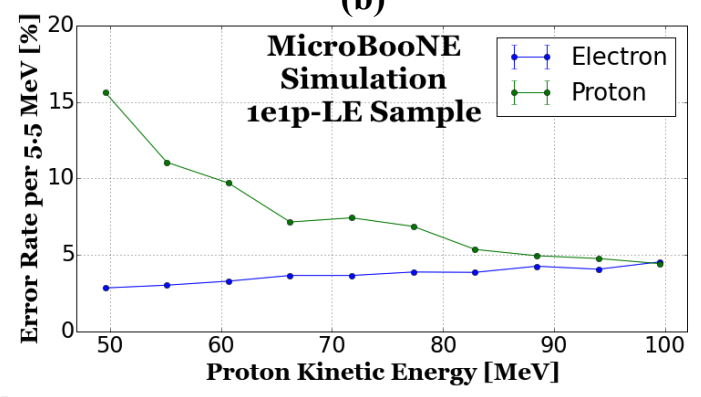

. 

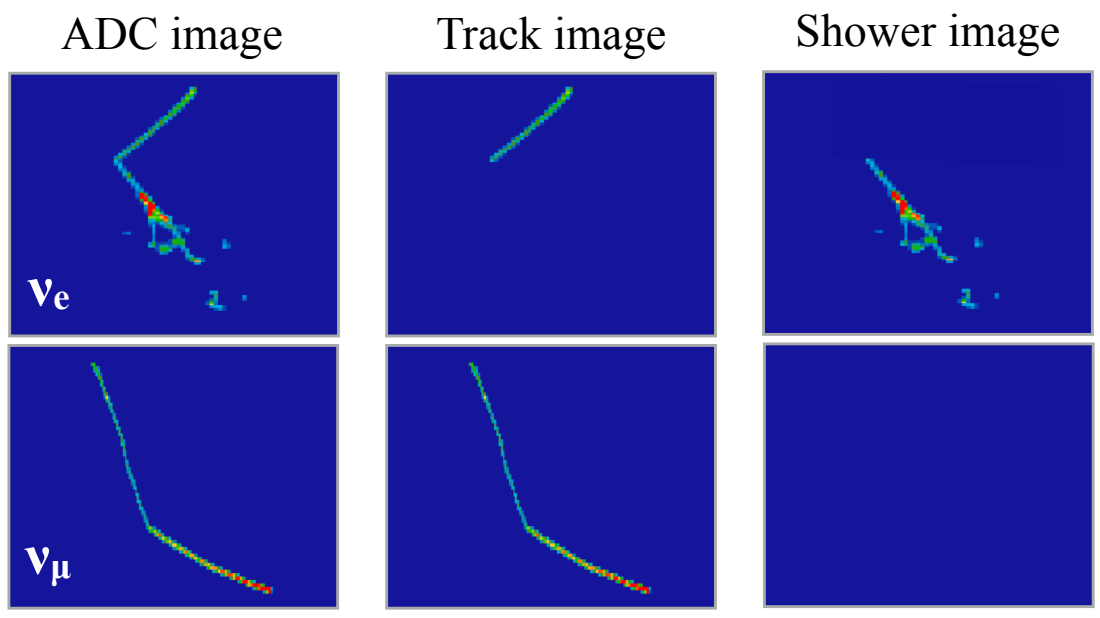

Figure 8.27: Three separate images are provided as input into the vertex finding algorithm. An ADC image which contains all pixels in the cROI. A track image which contains only track labelled pixels. A shower image containing only shower labelled pixels. Example inputs are shown for a $1 e 1 \mathrm{P}$ (top row) and $1 \mu 1 \mathrm{P}$ (bottom row) interaction.

is designed for dedicated reconstruction of tracks and showers separately where different algorithm techniques are be used. The algorithm then reconstructs combined features by considering all pixels together. In this design the algorithm is detached from having to algorithmically identify track and shower particles and can make different assumptions about how to locate the vertex for $1 e 1 \mathrm{P}$ interactions, which feature a single track and shower, and $1 \mu 1 \mathrm{P}$ interactions, which feature two tracks. By knowing the track and shower label before hand, the pattern recognition is simplified for finding the vee shape.

The algorithm is additionally supplied a set of images with tagged cosmic ray information and with images marking the spatial location of dead wires. The cosmic ray images contains through going muon pixels and stopping muon pixels tagged. In this reconstruction, through going pixels are erased from the image while stopping muon pixels are unused. With through going muon pixels removed from the ADC, track, and shower images, the probability that the algorithm will reconstruct cosmic background is greatly reduced. The dead wire image set marks the location with dead wires. This way the algorithm can know precisely which region in the image contains pixels which contain no charge and make a decision about whether to veto certain detector regions during reconstruction. 


\subsubsection{Track Vertex Finding}

To reconstruct $1 \mu 1 \mathrm{P}$ interactions, which features two track-like particles, the algorithm searches for a vertex in the track image. The algorithm searches for a vee shape in three dimensions by first analyzing 2D track clusters to identify "vertex seeds". Vertex seeds are $2 \mathrm{D}$ objects which represent potential projections of a $3 \mathrm{D}$ vertex. For example, a vertex seed can be a kink in a track which could represent an interface between two particles or the centroid of an object with any number of prongs emitted out of a single point. To identify vertex seeds an algorithm for breaking a generic track object and into straight line components was developed. An example of a non-trivial track object which is broken into unique clusters is shown in Fig. 8.28.
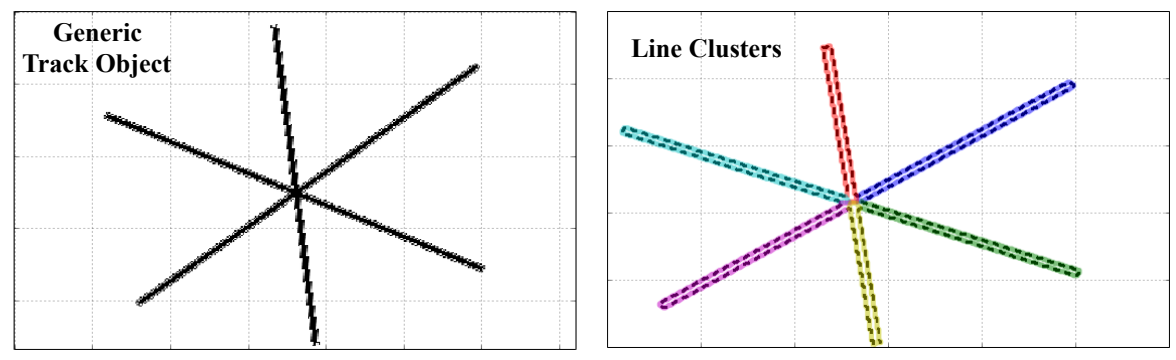

Figure 8.28: Left: Example of a star shaped object containing 6 obvious prongs. Right: Output of the algorithm. A collection of 6 straight clusters connected at a central point, or a potential vertex seed.

In Fig. 8.29 a LArCV image of a simulated $1 \mu 1 \mathrm{P}$ event is shown on the $\mathrm{V}$ plane. In this image the muon particle travels toward the upper left hand portion of the image and the proton toward the lower right. This event will serve as a demonstration of the track vertex finding algorithm.

In Step 1, a LArOpenCV image of a $1 \mu 1 \mathrm{P}$ interaction is shown.

In Step 2, two types of track clusters are identified by separating two different charge scales. A distinction between pixels in the Low Charge (LC) and High Charge (HC) regime as shown in Fig. 8.30 is performed. The boundary between LC and HC ADC count is a constant threshold per plane and is determined from a study of the pixel intensities of simulated protons. The minimum $\mathrm{HC}$ value for the $\mathrm{U}, \mathrm{V}$, and $\mathrm{Y}$ planes are set at $140 \mathrm{ADC}$, 

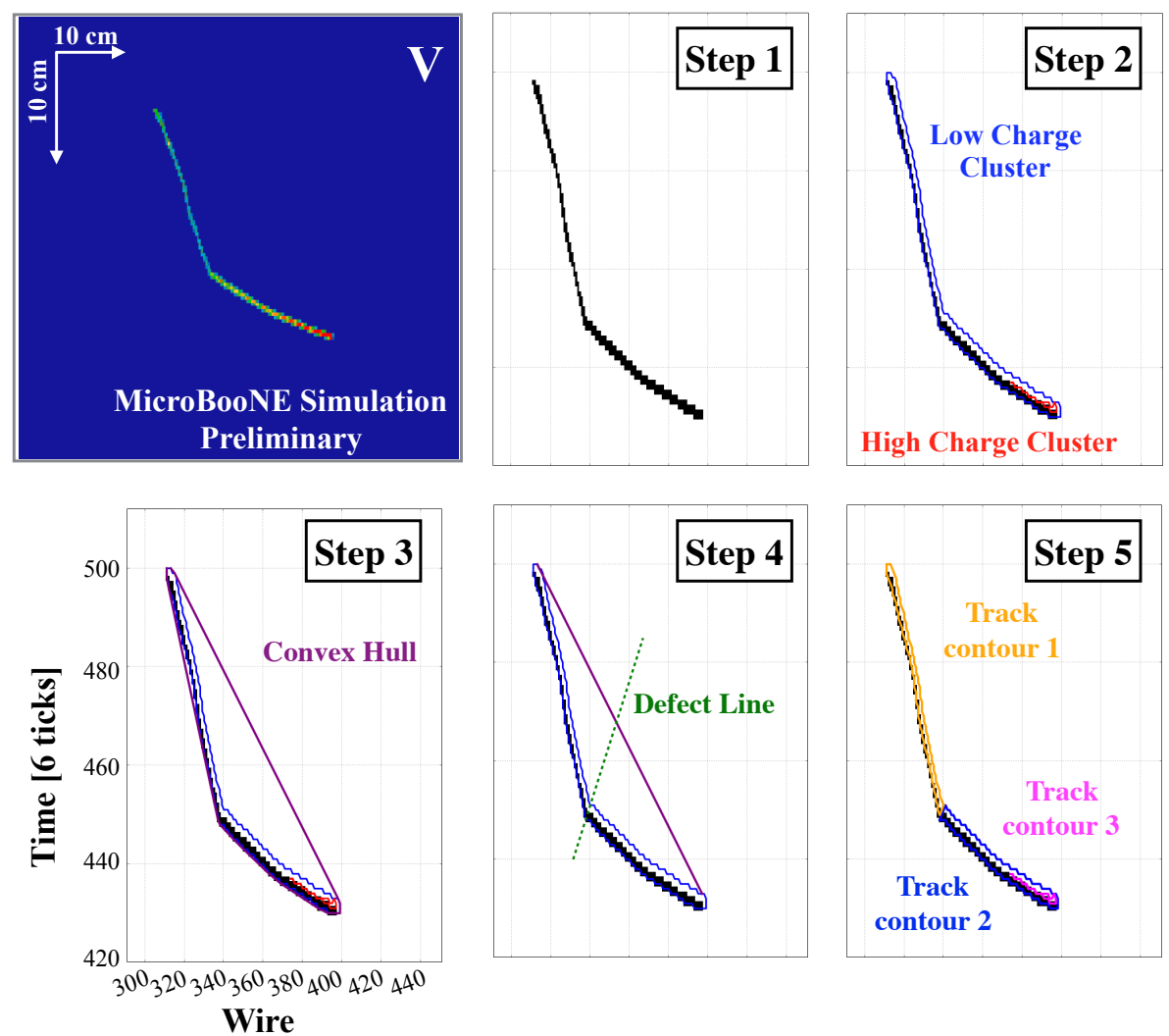

Wire

Figure 8.29: Algorithm steps 1 - 5, described in Section 8.6.2, for analyzing a $1 \mu 1 \mathrm{P}$ interaction to locate vertex seeds.

$120 \mathrm{ADC}$, and $80 \mathrm{ADC}$ respectively. These values correspond to $10 \%$ of the average pixel value for a proton track on each plane. Pixels with values below $10 \mathrm{ADC}$ count are not considered in the reconstruction.

Once the pixel ranges are separated, the algorithm finds groups of LC and $\mathrm{HC}$ pixels by applying the OpenCV contour finder. This step defines the LC and HC pixel clusters. Pixels that satisfy the HC condition are also included in the LC clusters, to avoid hollow clusters. HC clusters are therefore a subset of the LC clusters.

In Step 3 the vertex algorithm computes the convex hull, which is the smallest convex polygon which bounds the original cluster.

In Step 4, the algorithm identifies a defect point, which are the sides of the convex hull which are far away from their corresponding sides on the contour. The point on the contour 

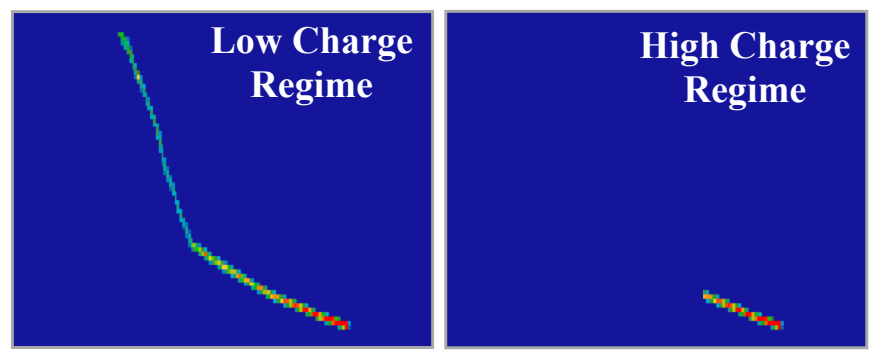

Figure 8.30: Distinction between low (left) and high (right) charge regimes for a $1 \mu 1 \mathrm{P}$ interaction. The low charge regime encapsulates all pixels in the image above 10 ADC. The high charge regime contains pixels above a higher charge threshold. In this example, only the proton bragg peak passes the HC threshold.

that is farthest away from the corresponding hull side is called the "defect point", and is a location where the cluster is potentially bending and changing direction. If the convex hull side is far enough away, 5 pixels, from the defect point the contour is then broken into two at that point. The bottom image shows the three clusters obtained after this stage, 1 $\mathrm{HC}$ cluster and $2 \mathrm{LC}$ clusters. The algorithm then iteratively breaks down all clusters into linear segments until no defect points remain.

The result are three straight track contours for the example $1 \mu 1 \mathrm{P}$ interaction shown in Step 5.

Next, two types of vertex seeds are identified in using the line clustered image as shown in Fig. 8.31 ,

The first set of vertex seeds are the location of the defect points and as shown in Step 6 of Fig. 8.31 .

The second set of vertex seeds is found using a Principal Component Analysis (PCA) procedure which fits the clusters to a straight line hypothesis. The PCA is a linear approximation which minimizes the perpendicular distance between the data (the pixel points), and the estimated line. A PCA is calculated for each line cluster separately. Since all clusters have been broken into linear segments by removing defects, a linear approximation is suitable. The algorithm then computes the intersection of all possible PCA lines on the plane. If the lines intersect near a location on the image with charge, the point is saved and 

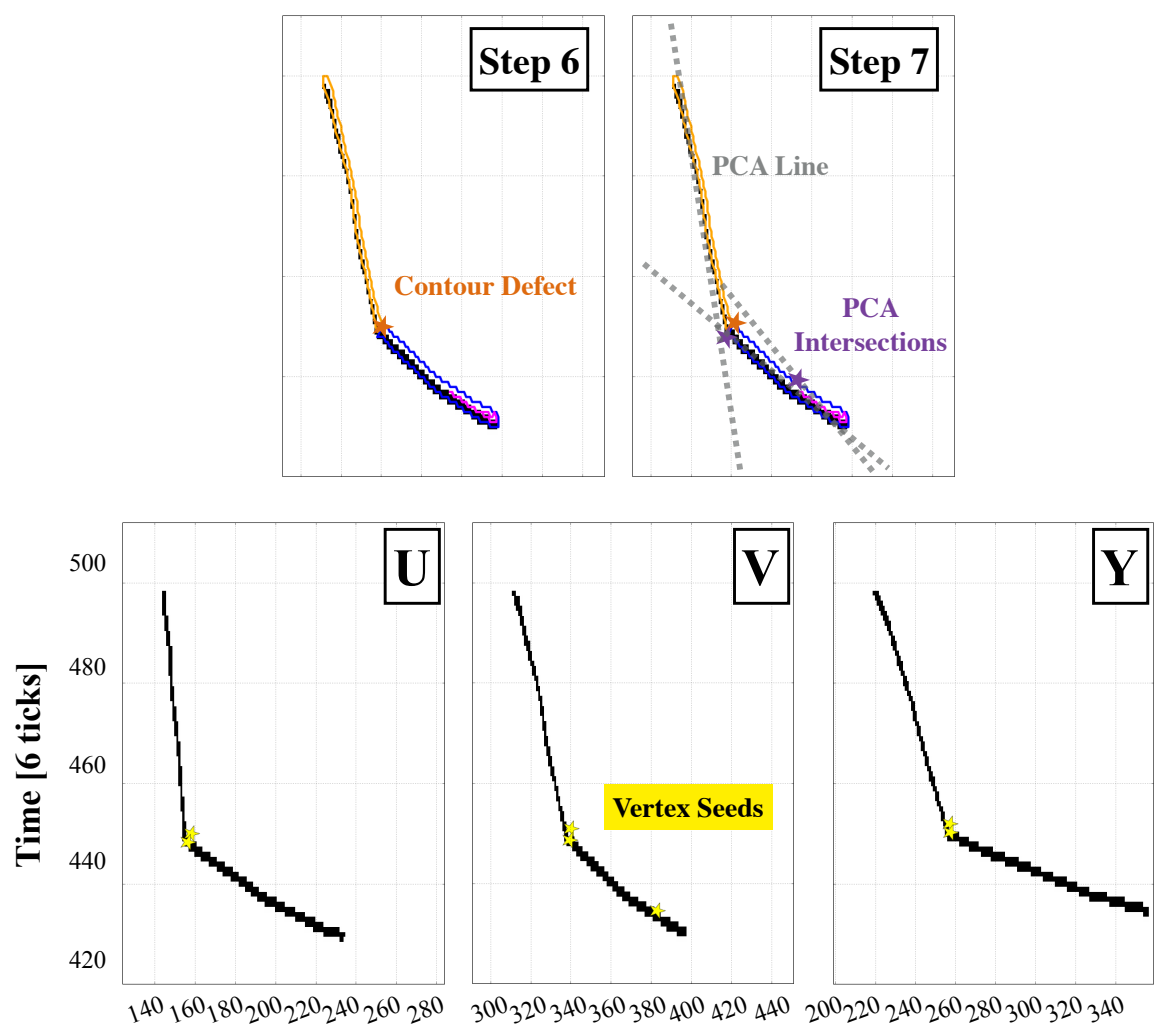

Wire

Figure 8.31: Algorithm steps $6 \&$, described in Section 8.6.2, for analyzing a $1 \mu 1 \mathrm{P}$ interaction to locate vertex seeds.

is added to the set of vertex seeds. Intersection points far from any charge are ignored. The upper right image in Fig. 8.31 shows the three PCAs found in the event example. Although three intersections points are found, only two correspond to pixels with charge and are then kept (middle image). This type of vertex seed helps find the 2D location where tracks which may be changing direction in the image. Also, using a linear approximation for the clusters provides an additional set of vertex seeds which increases the efficiency to find the $3 \mathrm{D}$ vertex.

The set of vertex seeds for the example $1 \mu 1 \mathrm{P}$ interaction is shown in the lower half of Fig. 8.31 for the U, V, and Y planes as yellow stars. One can notice a clustering of vertex seeds around the true projected neutrino scattering point where the muon and proton particle are produced. 
The algorithm then performs an exhaustive search for a 3D vertex around each seed by minimizing a quantify called the "angular metric" or $\mathrm{d} \Theta$ for short. The angular metric is a single quantity which measures the likeliness of two tracks being emitted radially outward from the same position across two or more planes. This metric is minimized when a point in $3 \mathrm{D}$ space is found where the $2 \mathrm{D}$ projections indicate that tracks are coming out of a single point.

The algorithm begins a search for a $3 \mathrm{D}$ vertex by defining a local search region around each vertex seed as shown in Fig. 8.32 a.
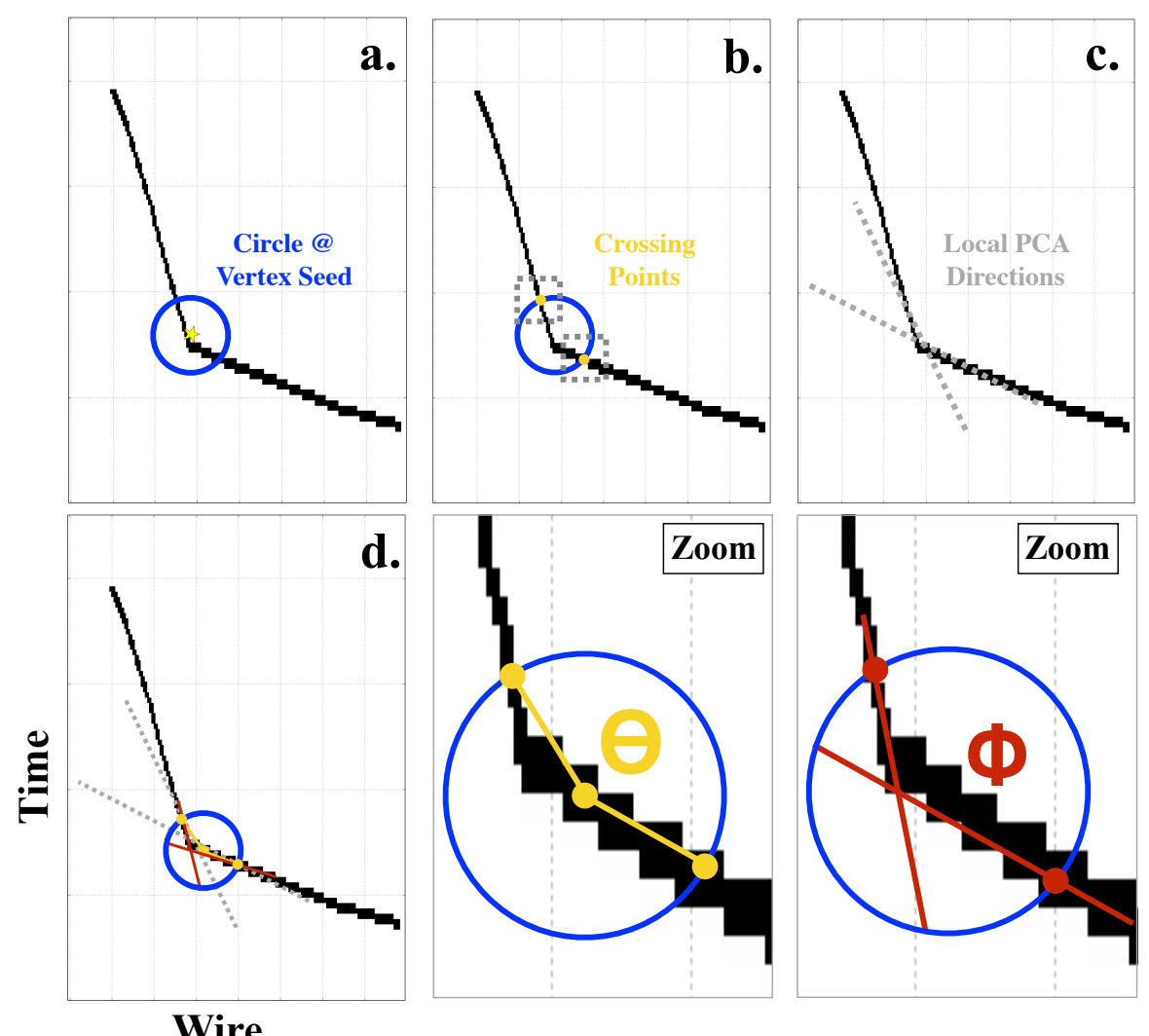

Wire

Figure 8.32: Algorithm steps a-d, described in Section 8.6.2, for analyzing a $1 \mu 1 \mathrm{P}$ interaction to locate the $3 \mathrm{D}$ vertex from vertex seeds.

Circles of radius 6 and 12 pixels are drawn with the given vertex seed at the center. The circle size with the greatest number of tracks coming out is used. In cases where the same number of tracks cross the circles, the largest circle is kept. The algorithm then identifies 
the points at which the out-going tracks cross the circle. In the example shown in Fig $8.32 \mathrm{~b}$ crossing points are found and are labelled as yellow dots. For each pair of out-going tracks, the algorithm evaluates two angular quantities:

1. The first angular quantity, $\Theta$, is the smallest angle between the center of the circle, and each pair of track-circle intersection points.

2. The second quantity, $\Phi$, is calculated in the following way. At each cluster-circle intersection point a small region of $7 \times 7$ pixels is identified. In this region, a PCA of charge carrying pixels is computed, and provides an estimate of the local track directions. $\Phi$ is the smallest angle between each local PCA pair.

The definition of the two angles are shown in the low half of Fig. 8.32 ,

Next, the location for which $\mathrm{d} \Theta$ is minimized is found. Starting at the vertex seed, two lines segments are drawn from the initial local PCA approximations as shown in Fig. 8.32. The circle center is then stepped in increments of 1 pixel along the straight line segments. The algorithm then computes the magnitude difference $\mathrm{d} \Theta=|\Theta-\Phi|$. When the local track direction on the circle boundary $(\Phi)$ matches the direction between the center and crossing points $(\Theta)$ the difference is small and indicates that the circle is at a location where the tracks are coming out straight from the center point, likely indicating a kink feature. At each step $\mathrm{d} \Theta$ is evaluated and stored per time tick. The stepping stops when the algorithm has scanned a $40 \times 40$ pixel region around the initial vertex seed. This procedure is repeated for each vertex seed in the plane. The evolution of $\mathrm{d} \Theta$ as a function of the corresponding time tick is represented at the bottom of Fig. 8.33 .

If two vertex seeds happen to scan the same time tick, the lowest $\mathrm{d} \Theta$ value is stored. Next, the $\mathrm{d} \Theta$ versus time-tick maps for the three views are summed to produce a single distribution of $\mathrm{d} \Theta$ values as shown in Fig. 8.34. The summed $\mathrm{d} \Theta$ profile is to be minimized to find the best vertex location. The distribution is smoothed using a rolling mean approximation of 6 time ticks. Finally the algorithm searches for local minima in the spectrum to find regions where a coincident vertex feature appears across multiple planes shown as the black vertical line in Fig. 8.34 .

In each plane, the circle at the local minima time is examined and matched across 


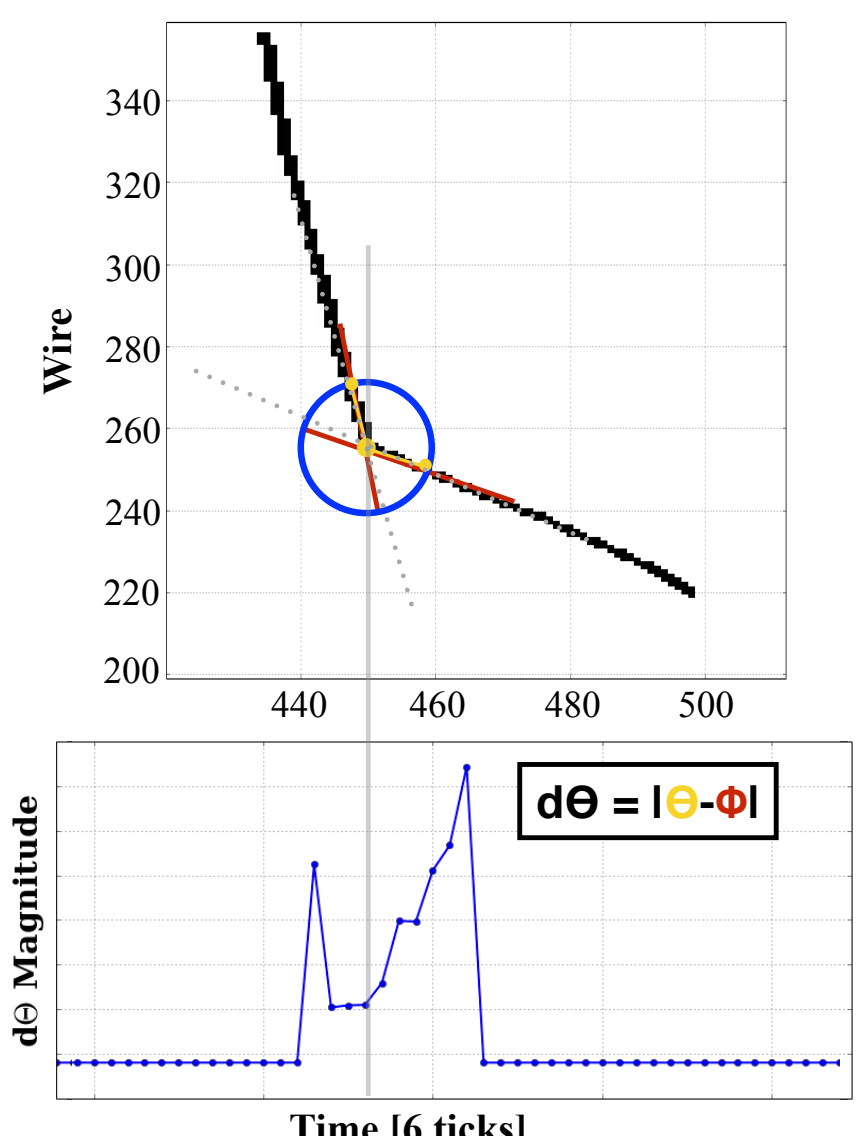

Figure 8.33: Procedure for scanning the collection of vertex seeds to search for the best kink location by minimizing $\mathrm{d} \Theta$ as described in the text.

planes using wire coincidence. If coincident wires are found, then a $3 \mathrm{D}$ vertex is claimed. An equivalent procedure is carried out by performing the search for local minima in the angular spectrum in overlapping wire regions.

To obtain the $3 \mathrm{D}$ vertex position, the vertex time provides the $\mathrm{X}$-coordinate. The $\mathrm{Y}$ and $\mathrm{Z}$ spatial information of the vertex is extracted from wire coincidence across any pair of $\mathrm{U}, \mathrm{V}$, and Y planes at the minimum time tick. Finally, the resulting 3D vertex is refined using a $3 \mathrm{D}$ volume scan in a $(4 \times 4 \times 4) \mathrm{cm}^{3}$ region around the vertex as described in Section 8.6.3. For each location in that space, the 2D projections of the point in $3 \mathrm{D}$ space are estimated, and the $\mathrm{d} \Theta$ variable is computed. This stage allows to further improve the estimation of the best $3 \mathrm{D}$ location of the vertex. The reconstructed $3 \mathrm{D}$ vertex location is 

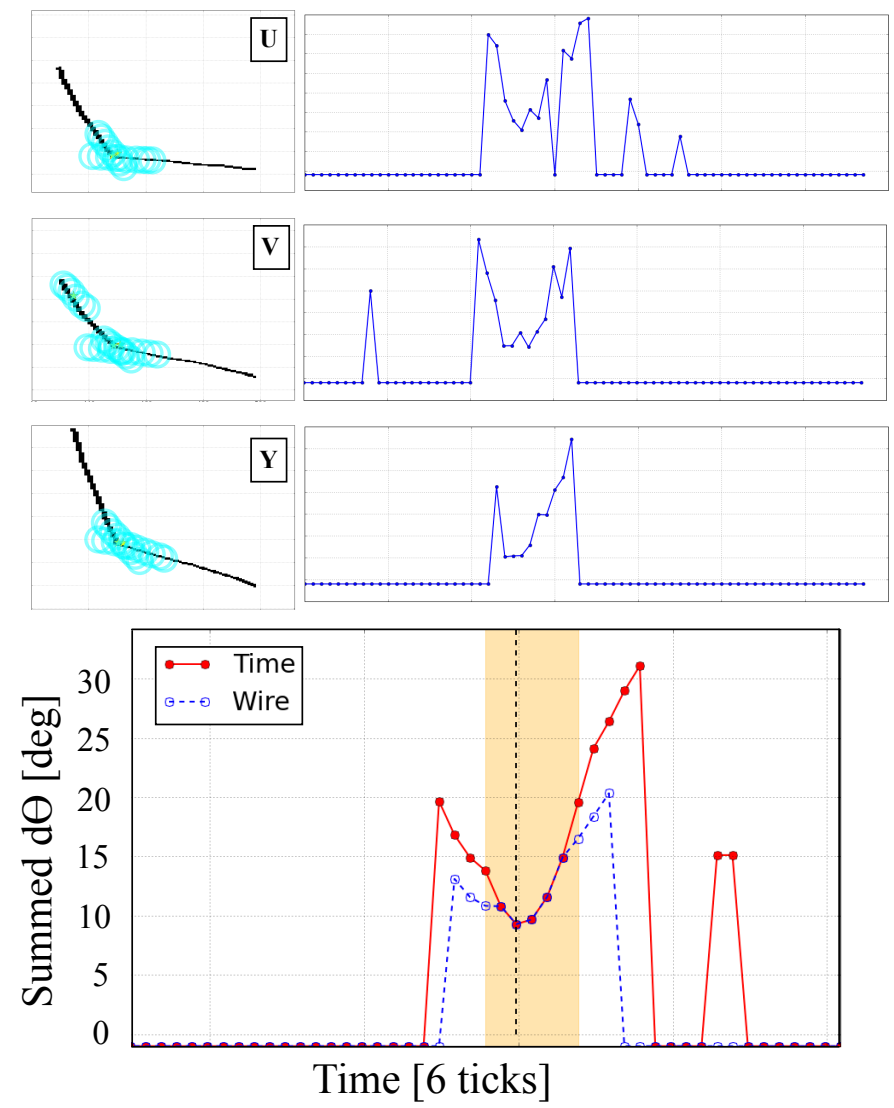

Figure 8.34: Top: Minimization of $\mathrm{d} \Theta$ performed for vertex seeds in each of the three wire planes. Bottom: Sum of $\mathrm{d} \Theta$ for coincident time tick and wire. A local minimum is found which defines a 3D consistent kink.

shown for the example $1 \mu 1 \mathrm{P}$ event in Fig. 8.35.

\subsubsection{Track - Shower Vertex Finding}

To reconstruct $1 \mathrm{e} 1 \mathrm{P}$ interactions the algorithm searches for a vee shape at the interface between track and shower pixels. An example SSNet image of a $1 e 1 \mathrm{P}$ interaction is shown in Fig. 8.36. Vertex finding is less complex than in the track-only case as two topologically different particles types are emitted from the vertex, one track and one shower. Since the track and shower pixels have been previously separated by the SSNet the algorithm can make an assumption about the straightness of track pixels. The vertex can be located on the edge of a single straight track connected to a cluster of shower pixels. Due to track and 


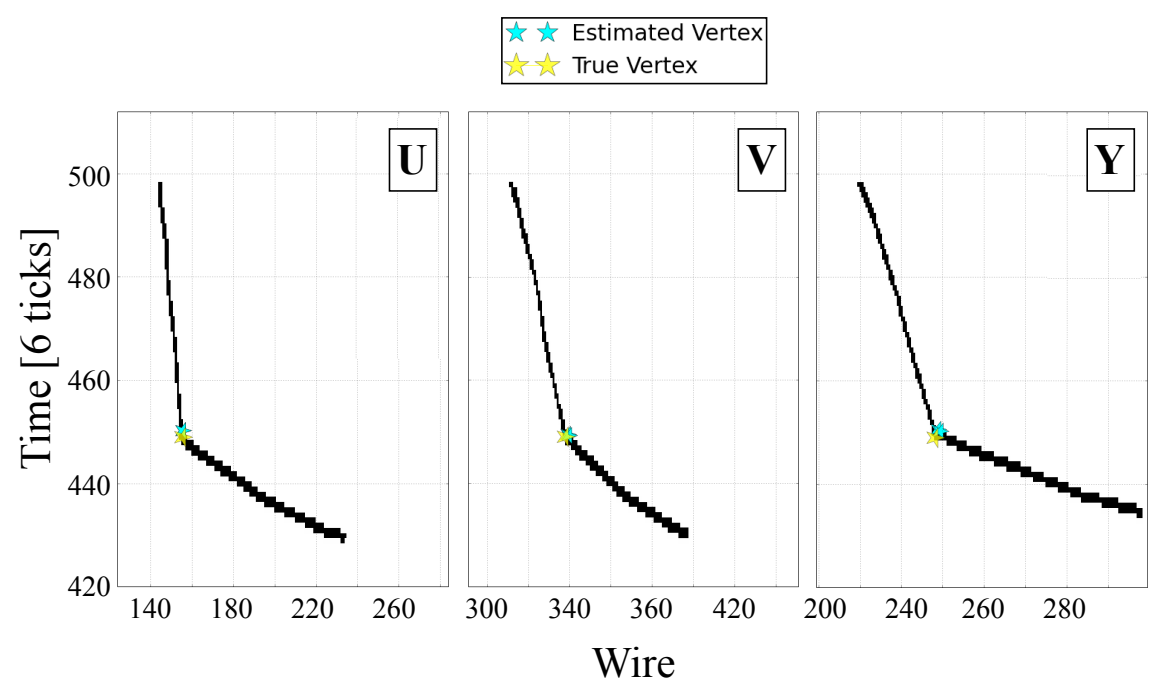

Figure 8.35: Reconstructed 3D vertex position (cyan) and true vertex position (yellow) for the example $1 \mu 1 \mathrm{P}$ interaction.

shower pixel classification near the true vertex point may not be precise, a kink point is optimized as in the case of track vertex finding.
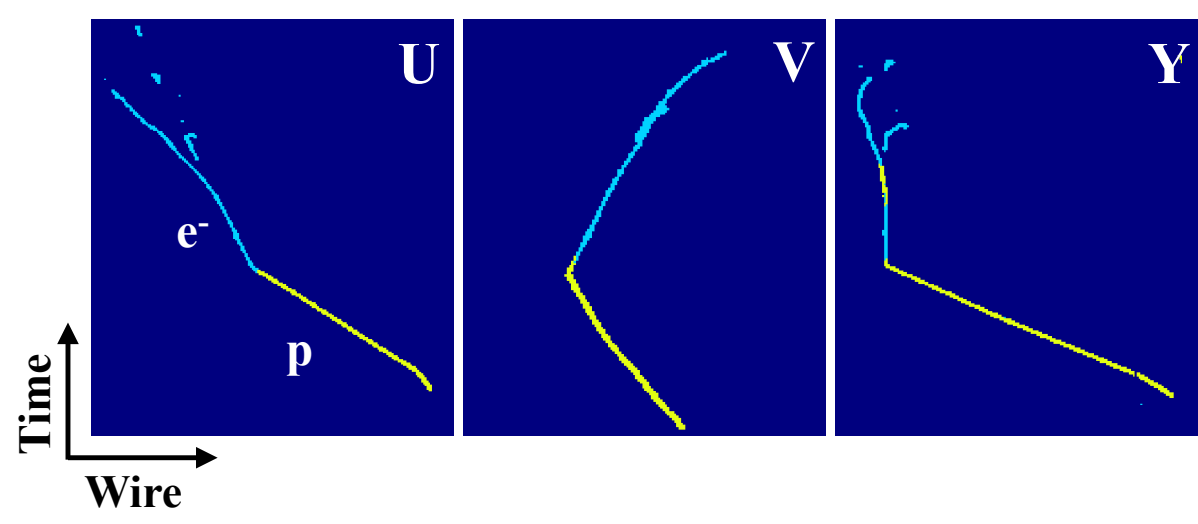

Figure 8.36: Example SSNet images of a 1e1P interaction to demonstrate the track-shower vertex finding algorithm. Track and shower pixels are labelled as yellow and cyan respectively.

First the algorithm identifies edge points in the track only image as shown in Step 1 of Fig. 8.37. Under the assumption that tracks are straight, edge points are found by finding the point farthest away from the mean position of all pixels of a cluster, then finding the 
second edge point by locating the furthest point away from the first edge. Each track cluster will have two $2 \mathrm{D}$ edge points.

At each edge point a circle of radius 10 pixels is drawn. In Step 2 the algorithm counts the number of times shower pixels cross the circle boundary. If zero points are found, no shower exists. If one or more crossing points are found, the track edge point likely does not contain a signal shower particle and is vetoed. To ensure that the shower pixels are physically connected to the track edge, a straight line is drawn between the vertex and the circle boundary. If a continous cluster of shower pixels lie on the line, then the shower crossing point is accepted. This ensures the shower particle is not a photon. In the example $1 e 1 \mathrm{P}$ interaction a single valid track-shower point is found on each of the three wire planes.
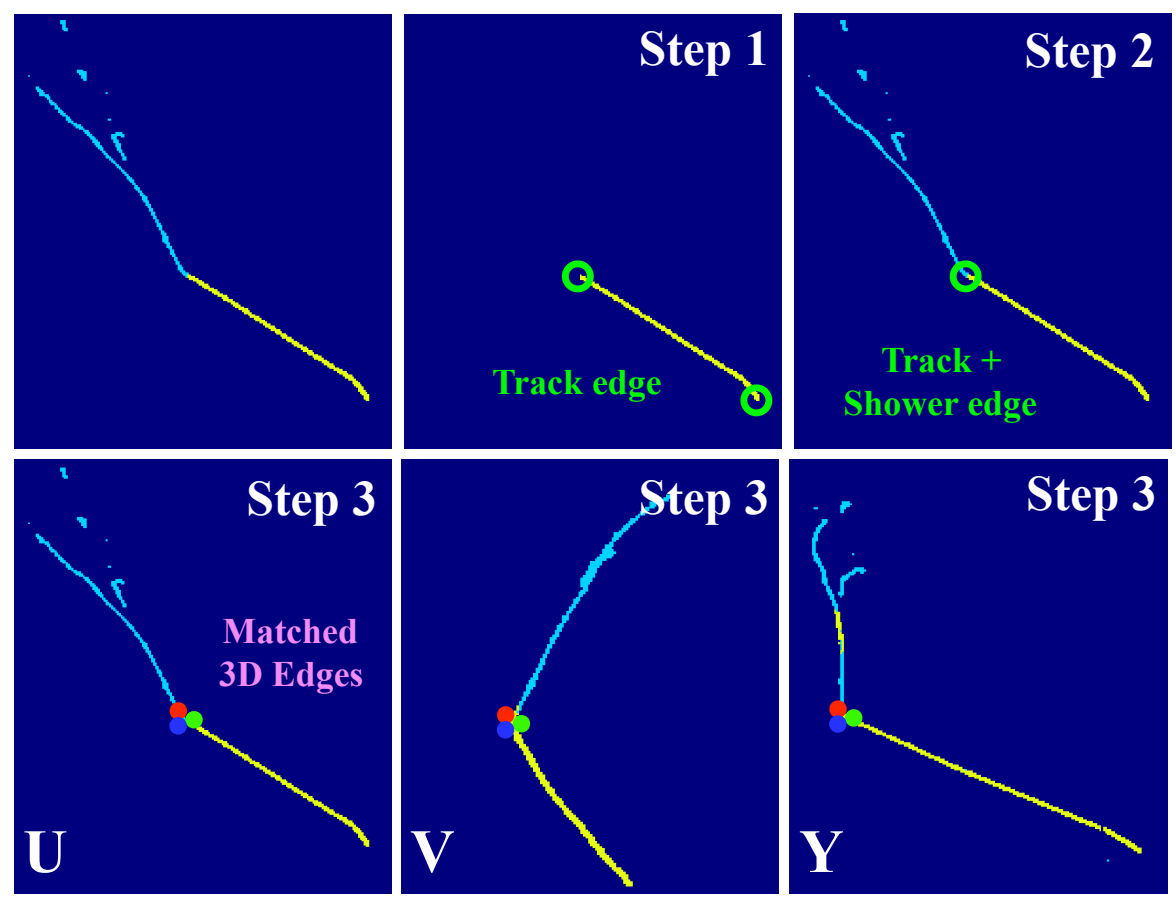

Figure 8.37: Algorithm step, described in Section 8.6.3, used to locate a consistent 3D edge where a shower is attached to a track.

Next, in Step 3, the algorithm compares the track-shower edge points pairwise across planes to form a candidate 3D point. A 3D point is formed for each pair of track-shower edges by matching the time and coincident wires. The $\mathrm{Y}$ and $\mathrm{Z}$ position of the candidate $3 \mathrm{D}$ point is formed by comparing coincident wires. The $\mathrm{X}$ position is determined as the 
average tick location between the two points. Candidate 3D point formation is rejected if the pair of track-shower edge points do not have overlapping wires or if the time coordinate of the pair are separated by 12 pixels $(\sim 4 \mathrm{~cm})$. Allowing for a generous time-coincidence between $2 \mathrm{D}$ edges helps handle the case where a shower exists on the end of a track which may not be consistent in three dimensions. This case may occur for instance when the trunk of the electron shower is classified as track pixels, and the number of trunk pixels classified as track varies across two or more planes, preventing a good $3 \mathrm{D}$ edge point to be found. In Fig. 8.37, three candidate 3D points are found and labelled as red, green, and blue.

To locate the single point in space where a vertex is likely present a grid scan is implemented to minimize the angular metric $\mathrm{d} \Theta$ in the space locally around each candidate point. At each 3D candidate point, a $4 \times 4 \times 4 \mathrm{~cm}$ volume is constructed as shown in Fig. 8.38 a. If two or more candidate points overlap in $3 \mathrm{D}$ space, the volumes are combined to form a mutual volume. This prevents the algorithm from repeatedly scanning the same 3D space. In Step 4 of Fig. 8.38 three 3D candidate points have been combined into one volume.
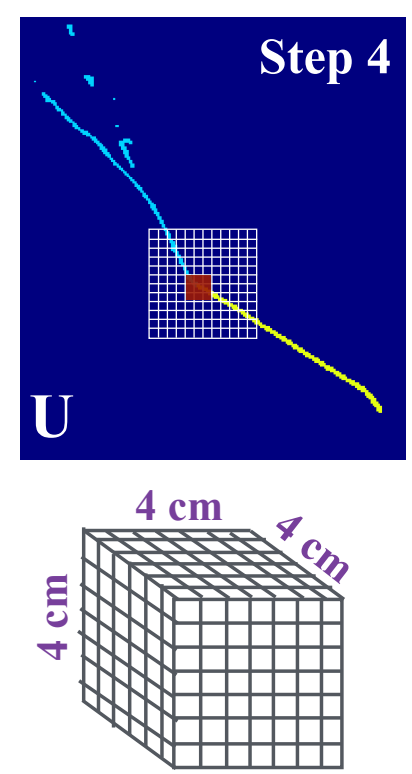

a.
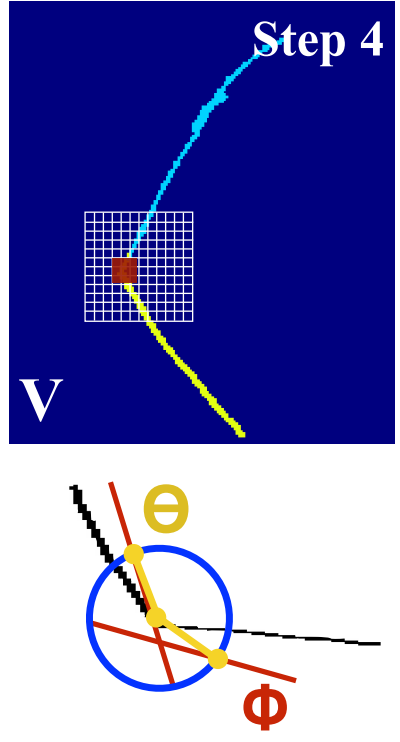

b.
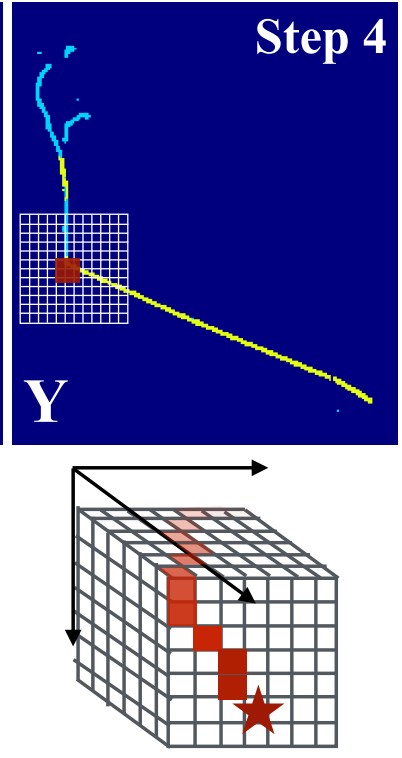

c.

Figure 8.38: The grid scan procedure for locating the best 3D interaction vertex for trackshower events. Steps are described in Section 8.6.3.

The grid is scanned in steps of $0.5 \mathrm{~cm}$ across the volume. At each $3 \mathrm{D}$ step in the volume 
a $2 \mathrm{D}$ point is formed by projecting onto the image. If the pixel is empty on two or more planes, the $3 \mathrm{D}$ point is rejected. Next, a circle is graduated in size from 6 pixels to 10 pixels in steps of 2 pixels. At each radial step the $3 \mathrm{D}$ angular metric $\mathrm{d} \Theta$ is computed. The product of $\mathrm{d} \Theta$ across planes is minimized over the $3 \mathrm{D}$ volume and the point with the lowest value is claimed as a $3 \mathrm{D}$ vertex candidate. By optimizing the product of $\mathrm{d} \Theta$ over multiple radii the best kink point location is found.
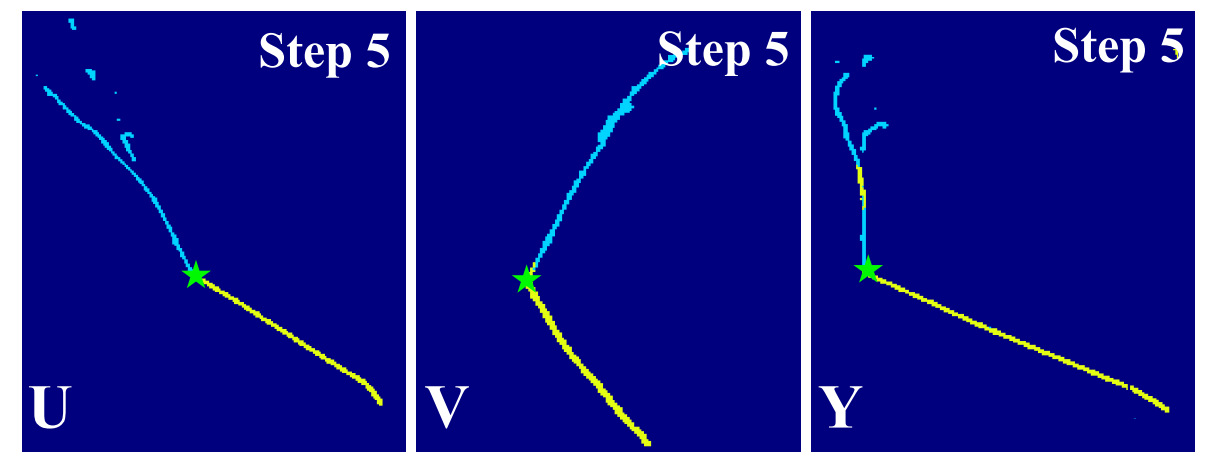

Figure 8.39: Location of the reconstructed $3 \mathrm{D}$ vertex (green star) for the example $1 e 1 \mathrm{P}$ interaction.

The result of the grid scan procedure for the example 1e1P event is shown in Fig. 8.39. Step 5 shows a single candidate vertex being located at the track and shower interface.

\subsubsection{Particle Clustering}

The algorithm performs a pixel clustering procedure to find unique particles coming out of each $3 \mathrm{D}$ vertex candidate. A particle is represented by a cluster of pixels found on two or more planes which represent the same energy deposition. First, a clustering algorithm groups unique sets of pixels together using the ADC image and the $3 \mathrm{D}$ vertex information. Finally, the algorithm matches plane-wise clusters across planes using temporal overlap to form particle objects.

The clustering procedure is applied to an example $1 \mu 1 \mathrm{P}$ event is shown in Fig. 8.403. For each reconstructed vertex the algorithm draws a circle of fixed size centered at the projected 2D point on each plane as shown in Fig. 8.40p.

The algorithm then locates where the pixels intersect the circle boundary. The clustering 

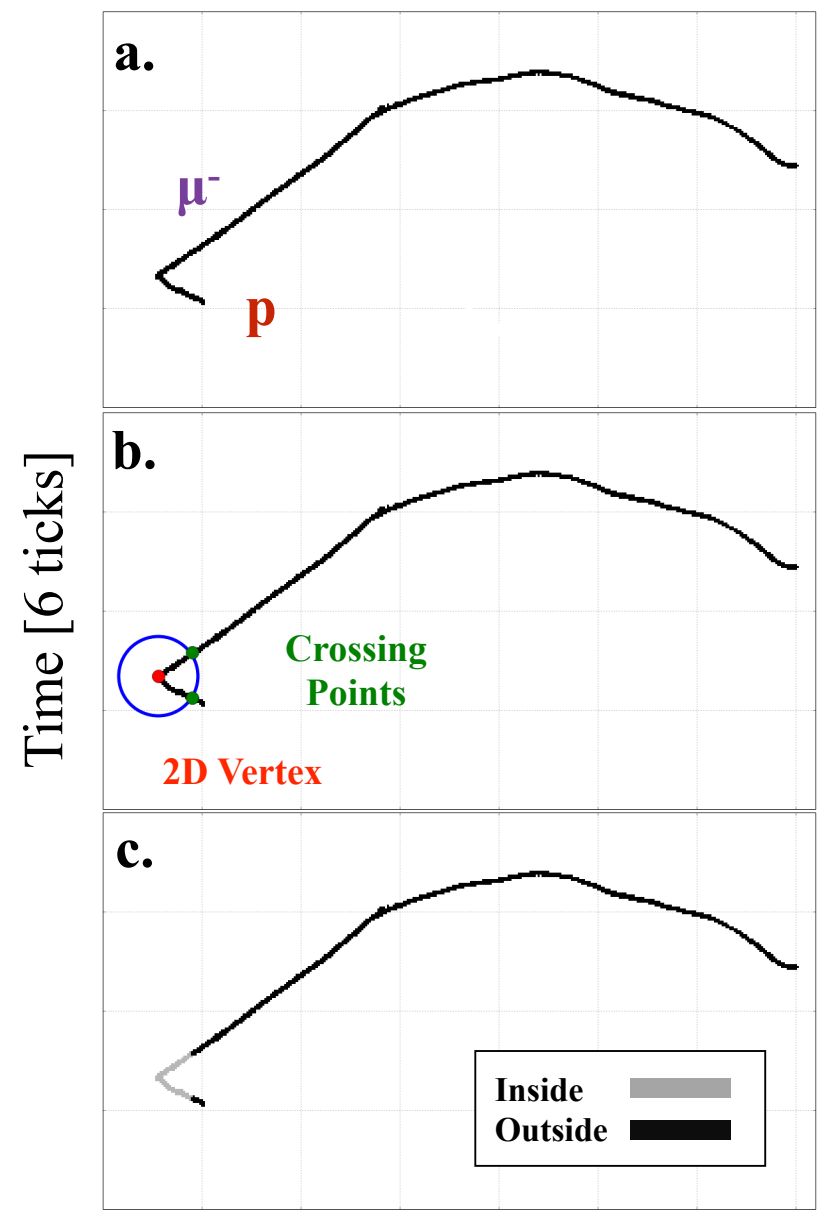

Wire

Figure 8.40: Particle clustering steps a-c for a $1 \mu 1 \mathrm{P}$ interaction as described in Section 8.6 .4 .

procedure is separated into two regions: inside and outside. Inside the circle the tracks emitted from the vertex point are straight, and outside they may exhibit some curvature as shown in Fig. 8.40.

First the algorithm considers only pixels inside the circle. A crop of the pixels inside the circle are shown in Fig. 8.413.

Pixels are transformed to polar space to be clustered. Polar space is a natural coordinate system to identify unique clusters coming out of the vertex since inside the circle clusters are locally straight and come out radially at fixed angle. Therefore, unique particles will be 

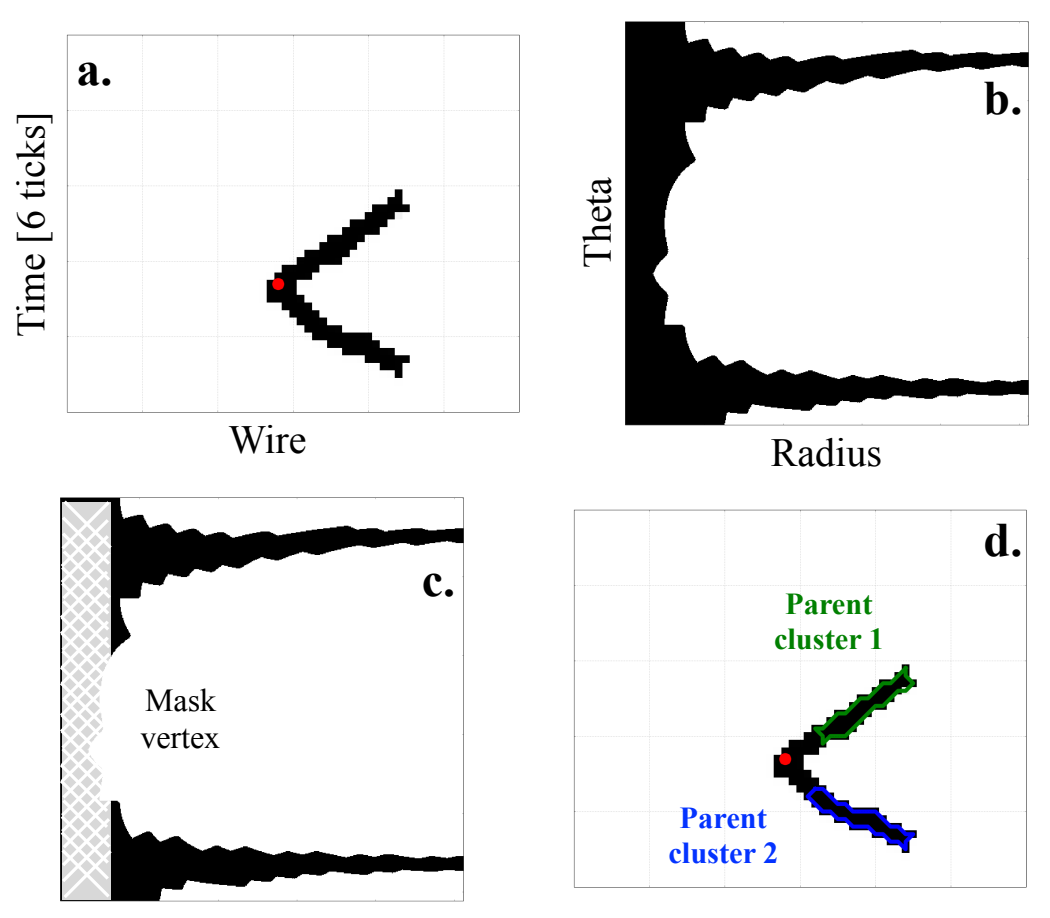

Figure 8.41: Particle clustering steps using a polar coordinate system described in Section 8.6 .4

be separated in the polar angle as shown in Fig. 8.41 b. To ensure particles are disconnected in polar space, a mask of 3 pixels is applied around the vertex, excluding those pixels from clustering as shown in Fig. 8.41k. In the polar space the algorithm finds two clusters at two separate polar angles. Both clusters are projected back into cartesian space as shown in Fig. 8.41 d.

Outside the circle unique clusters are identified in cartesian space to capture long range behavior. In the $1 \mu 1 \mathrm{P}$ example two child clusters are found in Fig. 8.42 b.

The algorithm then connects the parent clusters inside the circle with the child clusters on the outside by minimizing their distance to the circle boundary. This particle clustering procedure ensures that a unique cluster can be found near the vertex by using polar coordinates, and the cluster's long range behavior, which may be non linear, is captured as well.

Next, 3D consistent particles are formed from 2D clusters. The algorithm calculates the overlap in time of pairs $(\mathrm{U}, \mathrm{V}),(\mathrm{U}, \mathrm{Y}),(\mathrm{V}, \mathrm{Y})$ of plane clusters and assigns a score based 

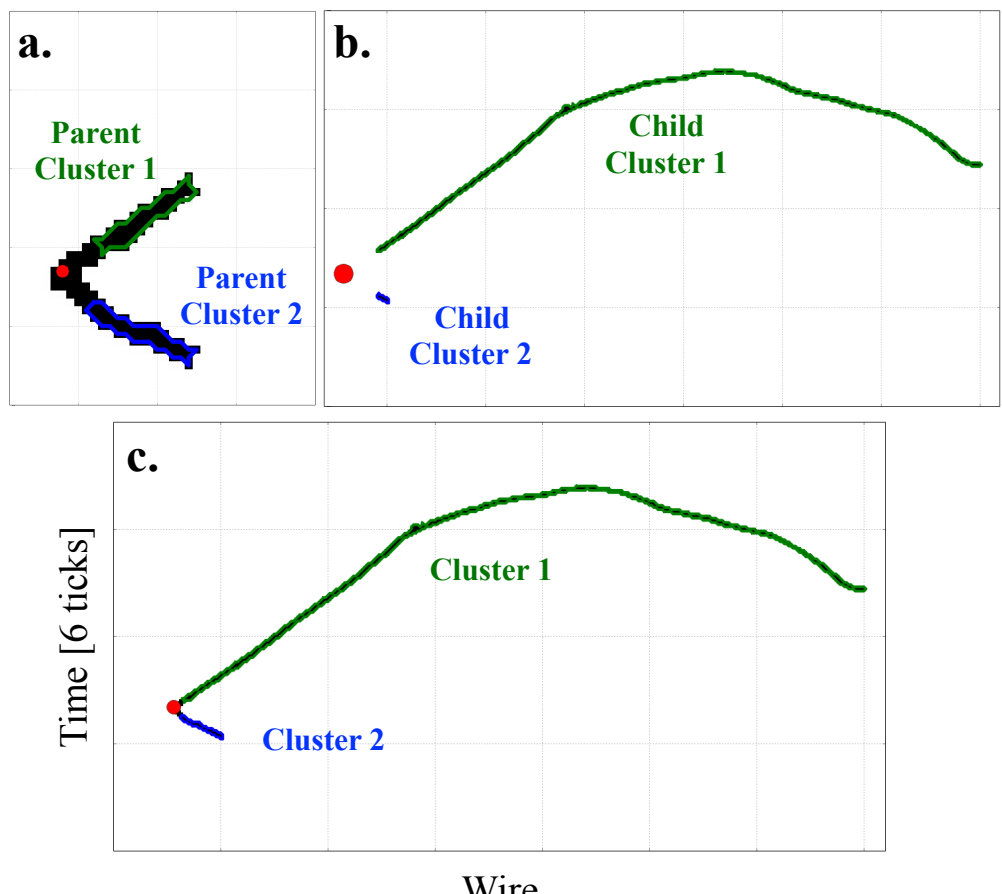

Wire

Figure 8.42: Particle clustering steps to connect parent clusters found inside the circle to clusters found outside. Two unique clusters are identified for the example $1 \mu 1 \mathrm{P}$ interaction.

on their overlap. Cluster pairs with the highest score are then associated with the same particle object. A figure of merit called the intersection over union, or IoU, measures the time interval common to two clusters over the union of the two clusters time interval. IoU is defined for two clusters A and B as,

$$
\mathrm{IoU}=\frac{\mathrm{T}_{\max }^{\mathrm{A}}-\mathrm{T}_{\min }^{\mathrm{B}}}{\mathrm{T}_{\max }^{\mathrm{B}}-\mathrm{T}_{\min }^{\mathrm{A}}}
$$

where max and min represent the largest and smallest time values of the cluster $\mathrm{A}$ and B. Cluster $\mathrm{A}$ is defined as the cluster which contains a pixels value later in time then cluster B. An IoU score of 1.0 signifies perfect overlap of clusters across planes. A minimum IoU of 0.1 is required to match two clusters, and cluster pairs are sorted in order of their score. If a cluster has a match with two or more clusters, the pair with the largest score is chosen. A triplet of clusters $(\mathrm{U}, \mathrm{V}, \mathrm{Y})$ is analyzed by computing their mutual pair wise IoU. If the difference between the maximum and minimum IoU is above threshold, the maximum IoU score is saved. The matching continues until no cluster-pairs with a match above the 
threshold value remain. An example of the IoU matching algorithm is shown in Fig. 8.6.4.

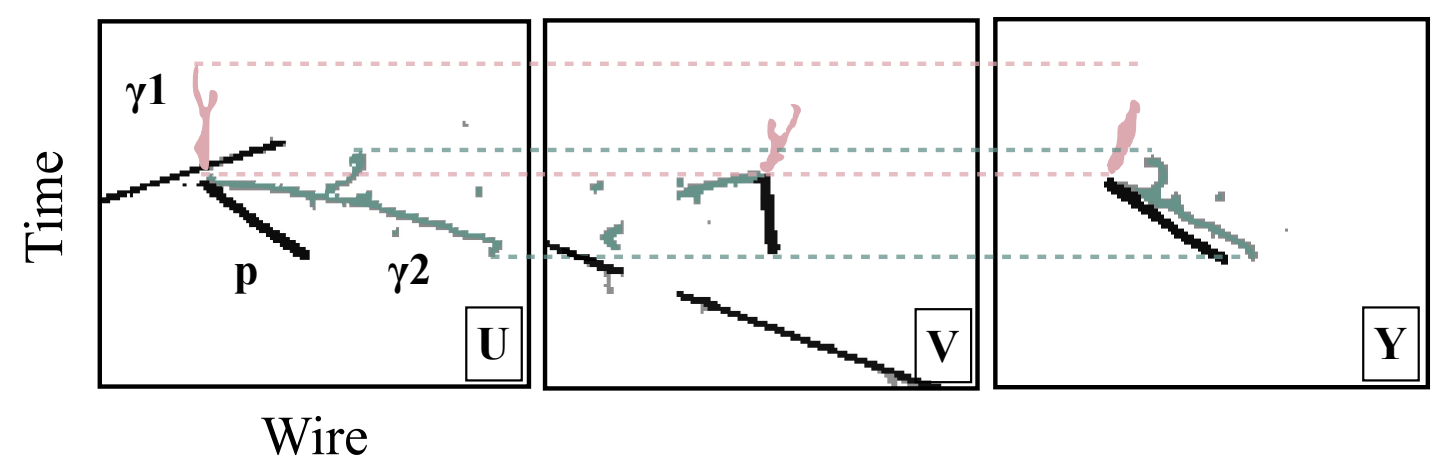

Figure 8.43: Example of the cluster matching procedure applied to a simulated neutrino event and described in Section 8.6.4.

In this example a vertex is found and three particles are identified: a proton (p), and two photons $(\gamma 1, \gamma 2)$ labeled as pink and green respectively. By comparing each clusters temporal extent across planes, denoted with pink and green dashed lines, clusters which share a common charge deposition are identified. A vertex must have at least 1 particle successfully reconstructed to be considered a candidate vertex.

\subsubsection{Vertex Resolution}

The quality of the track and track-shower image vertex-finding can be assessed using Monte Carlo by considering $\Delta \mathrm{R}$, the distance between the simulated true neutrino vertex to the reconstructed vertex. The $\Delta \mathrm{R}$ distribution for $1 \mu 1 \mathrm{P}$ and $1 e 1 \mathrm{P}$ events is shown in Fig. 8.44.

Monte carlo $1 \mu 1 \mathrm{P}$ and $1 e 1 \mathrm{P}$ events are required to interact inside the $\mathrm{TPC}$ active volume region and are reconstructed in the absence of cosmic rays. For $1 \mu 1 \mathrm{P}$ events $68 \%$ of vertices are reconstructed within $0.9 \mathrm{~cm}$ (3 pixels) of the true vertex. For $1 e 1 \mathrm{P}$ events $68 \%$ of vertices are reconstructed within $1 \mathrm{~cm}(\sim 3$ pixels $)$. Both topologies exhibit some fraction of reconstructed vertex $3 \mathrm{~cm}$ (10 pixels) or larger. These events are primarily associated with reconstructed vertex found on $3 \mathrm{D}$ kink points associated with proton scatters and on muon decay to michel electrons. 

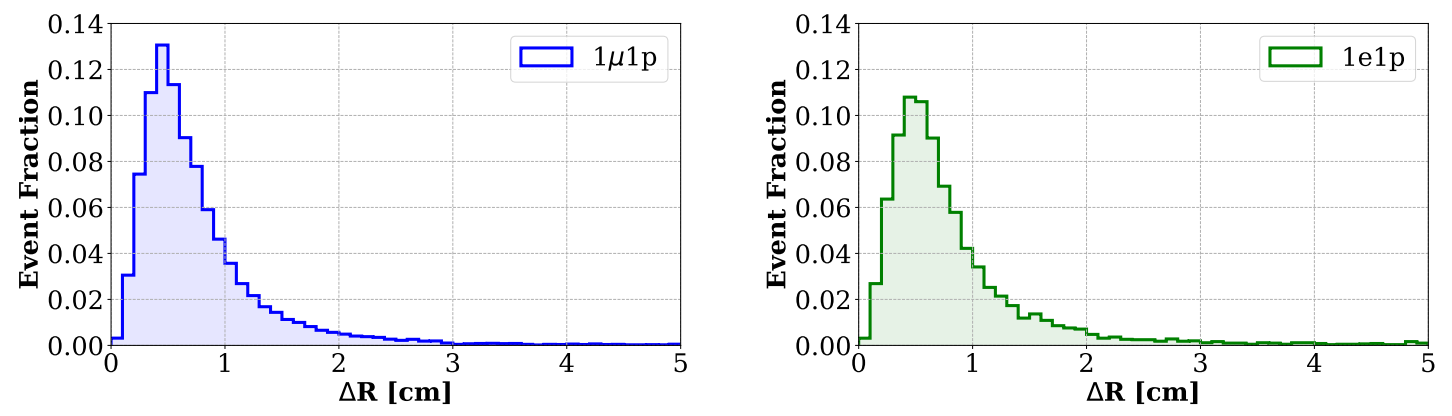

Figure 8.44: Distribution of vertex resolution $(\Delta \mathrm{R})$ for $1 \mu 1 \mathrm{P}$ (left) and $1 e 1 \mathrm{P}$ (right) events.

\subsubsection{Vertex Efficiency}

The vertexing algorithm is sensitive to a number of upstream factors which impact the ability to find a consistent 3D vertex across planes. Three factors are analyzed separately to determine their impact on vertex reconstruction efficiency as a function of true neutrino energy. The three factors are:

- Dead wires \& cROI Location

- SSNet pixel classification

- Cosmic Pixel Tagging

To decouple these effects on the vertexing algorithm each upstream factor will be applied individually to a Monte Carlo sample of 1L1P events without the presence of cosmic rays. All simulated events are located inside the TPC active volume. The true neutrino energy distribution for $1 \mu 1 \mathrm{P}$ and $1 e 1 \mathrm{P}$ events before any reconstruction is applied are shown in Fig. 8.45.

First, if the true neutrino vertex lies in or within 5 pixels of a dead wire region across two or more planes (the typical algorithmic circle size) then neutrino induced pixels can not be vertexed. Second, the neutrino can only be searched for inside a well reconstructed cROI, such that the cROI contains the neutrino vertex in at least two planes. A cROI is "correct" if it contains the true neutrino vertex position. Finding both the correct cROI, and the true neutrino vertex being located at least 5 pixels away from a dead wire in at 

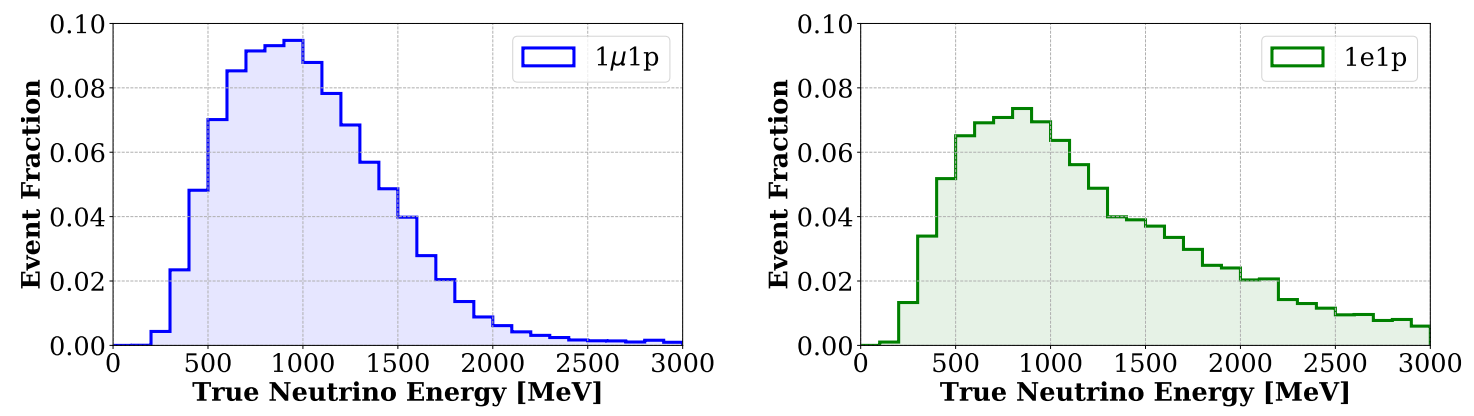

Figure 8.45: True neutrino energy distribution are shown for $1 \mu 1 \mathrm{P}$ (left) and $1 e 1 \mathrm{P}$ (right) events.

least 2 plane is called a "Good Event". The Good Event efficiency for 1L1P events as a function of true neutrino energy is shown in Fig. 8.46.
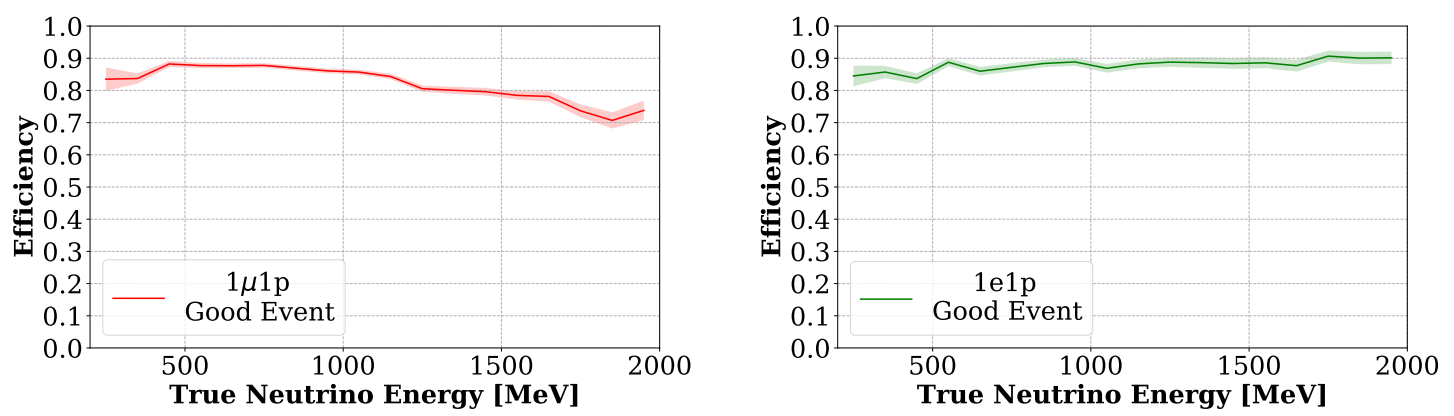

Figure 8.46: Good Event efficiency as a function of true neutrino energy for $1 \mu 1 \mathrm{P}$ (left) and $1 e 1 \mathrm{P}$ (right) events. Approximately $90 \%$ of events feature the interaction vertex inside a cROI and at least 5 pixels away from dead wires.

The Good Event efficiency is the ratio of number of Good Events to all simulated events. For both $1 \mu 1 \mathrm{P}$ and $1 \mathrm{e} 1 \mathrm{P}$ events the Good Event efficiency has no significant energy dependence. The efficiency is between $85 \%$ and $90 \%$ for both samples. To study the spatial dependence of the efficiency, the detector space is binned into $25 \mathrm{~cm}$ regions in the $\mathrm{Z}$ direction and $5 \mathrm{~cm}$ in the $\mathrm{Y}$ direction. An efficiency map over the detector for finding a Good Event is shown in Fig. 8.47.

A Good Event depends primarily on a vertex's spatial location to dead wires. The largest population of coincident dead wires is found below $200 \mathrm{~cm}$ in the $\mathrm{Z}$ direction, and 


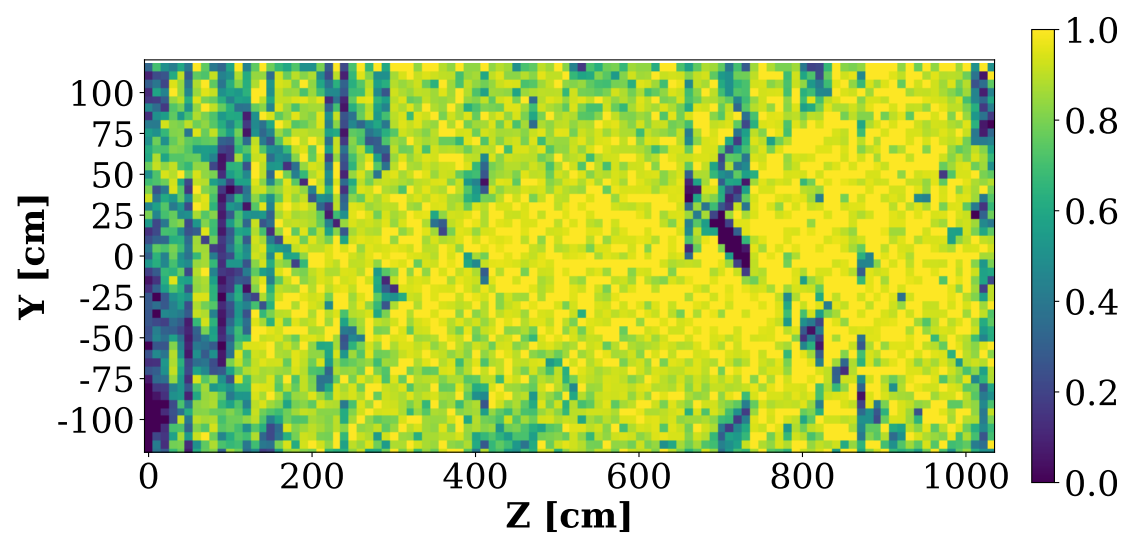

Figure 8.47: Spatial distribution of the Good Event efficiency binned in the Y-Z detector space.

in a region between 600 and $800 \mathrm{~cm}$. In these regions the dead wire inefficiency dominates and the Good Event decreases below 50\%. In regions where at least two planes feature good wires, such as between 400 and $600 \mathrm{~cm}$, the cROI finding inefficiency is dominant. In these region the Good Event efficiency is between $95 \%$ and $100 \%$. Averaged over the entire detector, approximately $10 \%$ of events will be unable to be vertexed using this algorithm.

With the Good Event efficiency deconvolved, we can study the reconstruction efficiency of vertexing algorithm given optimal inputs. The vertexing efficiency is defined as the ratio of events which contain a vertex within $5 \mathrm{~cm}$ of the true vertex to the total number of events. The second factor which can effect the vertex algorithm efficiency is the SSNet pixel classification. To study this effect we study the vertexing algorithm efficiency given perfect track and shower labelling using Monte Carlo information, called "Perfect Segment", and then turning on the reconstructed pixel labelling called, called "SSNet". The result is shown in Fig. 8.48 .

In this study, the inefficiency for a Good Event is deconvolved such that each MC event is a Good Event. For $1 \mu 1 \mathrm{P}$ events the vertex reconstruction efficiency for Perfect Segment rises from approximately $70 \%$ in the sub $500 \mathrm{MeV}$ region to approximately $80 \%$ above 750 $\mathrm{MeV}$ in true neutrino energy. In the lowest energy bin, the efficiency is approximately $60 \%$. When the SSNet is used a global decrease of $5 \%$ efficiency is observed. This means that 

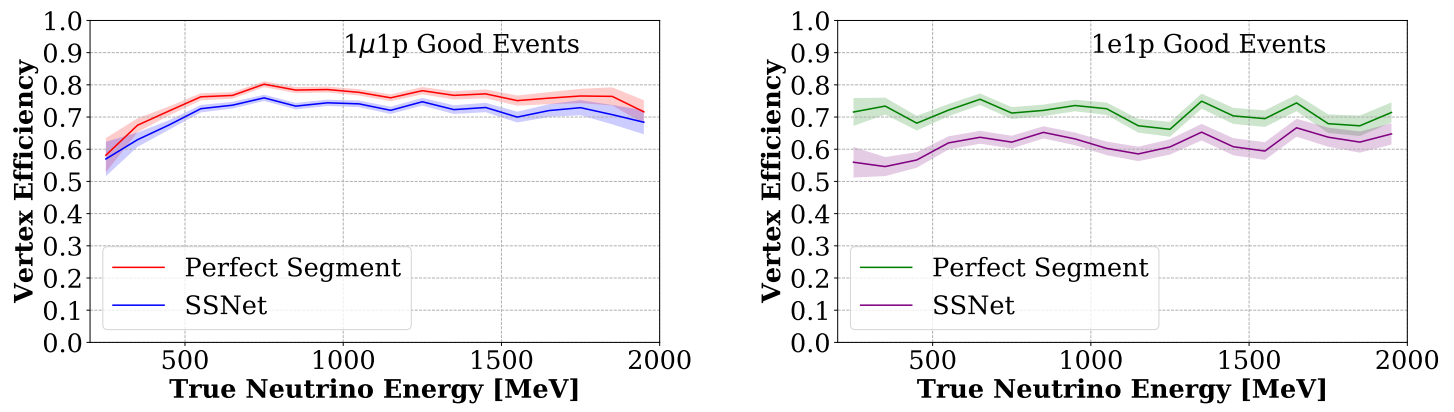

Figure 8.48: Vertexing efficiency as a function of true neutrino energy for $1 \mu 1 \mathrm{P}$ (left) and $1 e 1 \mathrm{P}$ (right) Good Events. The upper line in each plots shows the vertexing efficiency for perfect pixel labelling and the lower line shows the efficiency for using the SSNet pixel label.

the SSNet is classifying muon and proton pixels correctly as track with good performance. For $1 e 1 \mathrm{P}$ the vertex reconstruction efficiency for Perfect Segment is approximately $70 \%$ and flat over true neutrino energy. When the SSNet is applied a global decrease of $10 \%$ is observed. This is primarily due to a misclassification of electron trunk as track type particle inconsistently across planes causing the shower on track edge feature to be inconsistent in 3D. An example is shown in Fig. 8.49. The difference between Perfect Segment and SSNet is important in the near vertex region where the pixel classification determines which method to search for the vertex.

The final upstream factor which can effect the vertexing algorithm performance are from the cosmic ray tagging algorithms called ThruMu, and StopMu. ThruMu removes likely cosmic ray pixels which pass through the detector. The second cosmic tagging algorithm called StopMu is turned off by default in the reconstruction chain and is not analyzed here. The ThruMu algorithm is not completely efficient at removing all thorough going cosmic rays and can interfere with neutrino induced pixels. When this occurs, the neutrino pixels are labelled as cosmic rays, removed from the image, and no vertex can be reconstructed. An example of the negative effect of the ThruMu algorithm can be seen in Fig. 8.19 for an example $1 e 1 \mathrm{P}$ event. To study the effect of the ThruMu cosmic tagging algorithm on the vertex reconstruction efficiency we turn on ThruMu tagging in the case of the Perfect Segment image. The result is shown in Fig. 8.50 , 


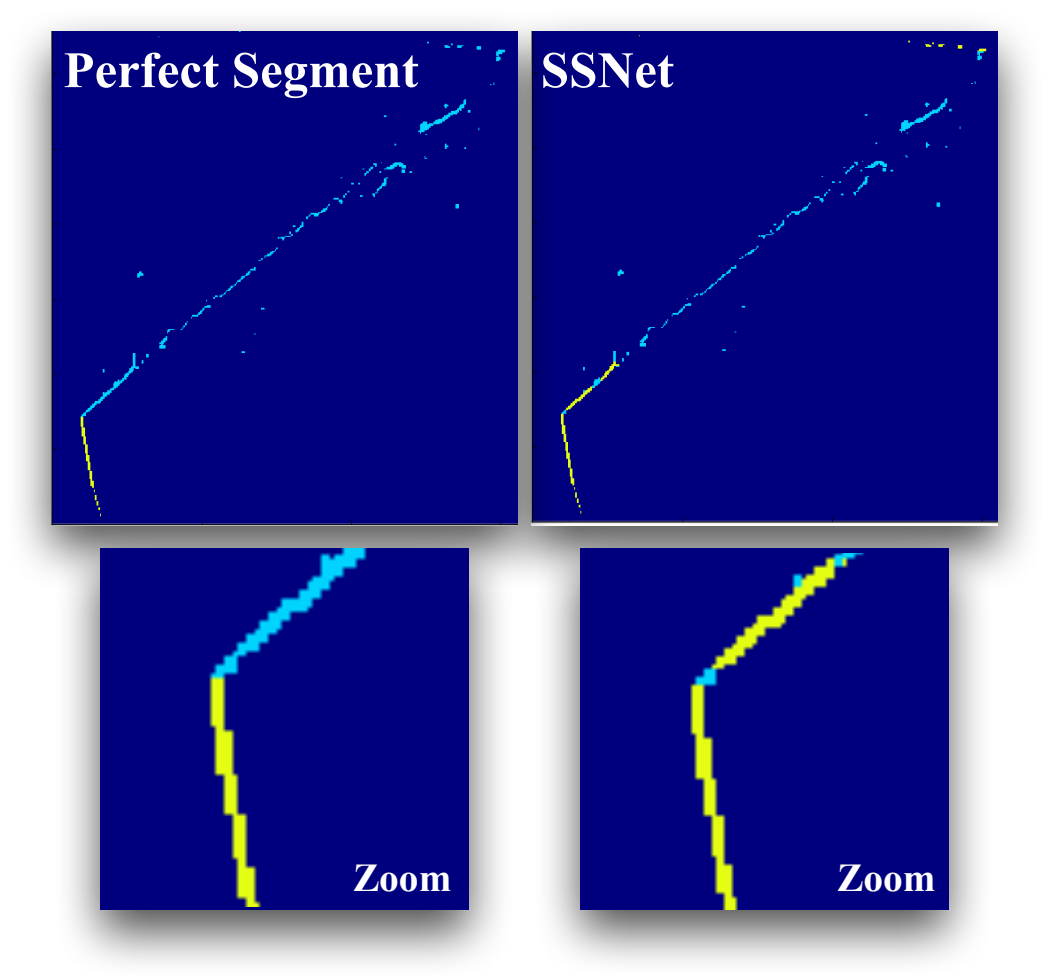

Figure 8.49: A 1e1P interaction with Perfect Segment (left), and SSNet (right). Track and shower pixel are labelled as yellow and cyan respectively. A zoom of the vertex region is shown in the bottom portion of the figure. A mis-classification of electron pixels as track in the vertex region removes the presence of a shower on track edge feature.

For the $1 e 1 \mathrm{P}$ sample we can expect approximately a flat $5 \%$ drop across all true neutrino energy. The $1 \mu 1 \mathrm{P}$ sample exhibits an energy dependent feature with respect to applying the cosmic tagger. As the neutrino energy increases above $750 \mathrm{MeV}$ the vertex reconstruction efficiency with cosmic tagging enabled decreases from $70 \%$ to $60 \%$. This feature can be attributed an increase in the muon daughter particle length. As the muon particle energy increases the muon track can exit the detector, this can cause the cosmic ray tagger to tag the neutrino as cosmic rays.

Additionally, the vertex algorithm is sensitive to parameters which affect the topology of the neutrino around the vertex point. In this section the reconstruction efficiency is studied as a function of simulation parameters which directly impact vertex finding. The simulation parameters are: 

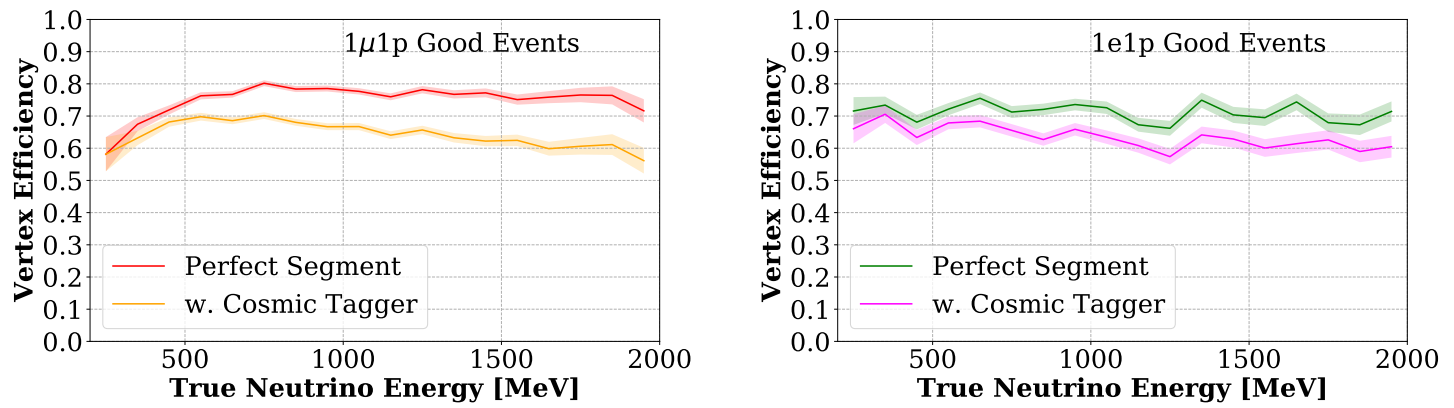

Figure 8.50: Vertexing efficiency for $1 \mu 1 \mathrm{P}$ (left) and $1 e 1 \mathrm{P}$ Good Events (right) as a function of true neutrino energy. The top line in each plot represents the vertexing efficiency for Perfect Segment. The bottom line represents turning on the ThruMu cosmic ray tagging algorithm.

- 3D opening angle between the lepton and proton (Fig. 8.51)

- Projected length on the Y plane of proton (Fig. 8.52)
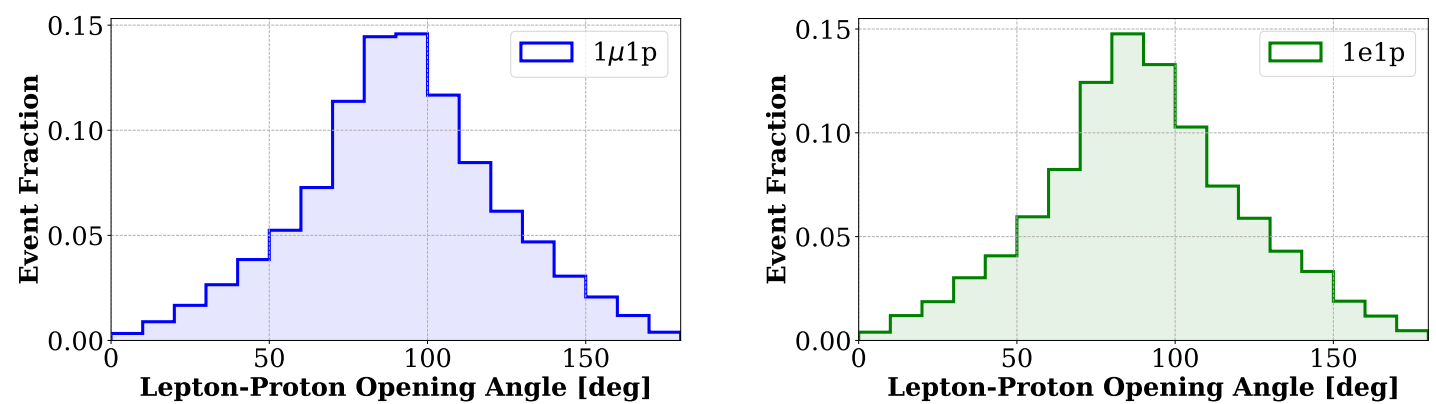

Figure 8.51: Opening angle distribution between the lepton and proton for $1 \mu 1 \mathrm{P}$ (left) and $1 e 1 \mathrm{P}$ (right) events.

The 3D opening angle between the lepton and proton is a proxy for the degree by which the vee shape topology is expressed in the image. The efficiency of the vertexing algorithm as a function of the lepton-proton opening angle using perfect segmentation for Good 1L1P events is shown in Fig. 8.53 .

Both $1 \mu 1 \mathrm{P}$ and $1 e 1 \mathrm{P}$ events feature a similar efficiency profile. When the lepton and proton are co-linear and span an angle less than approximately 30 degrees the efficiency 

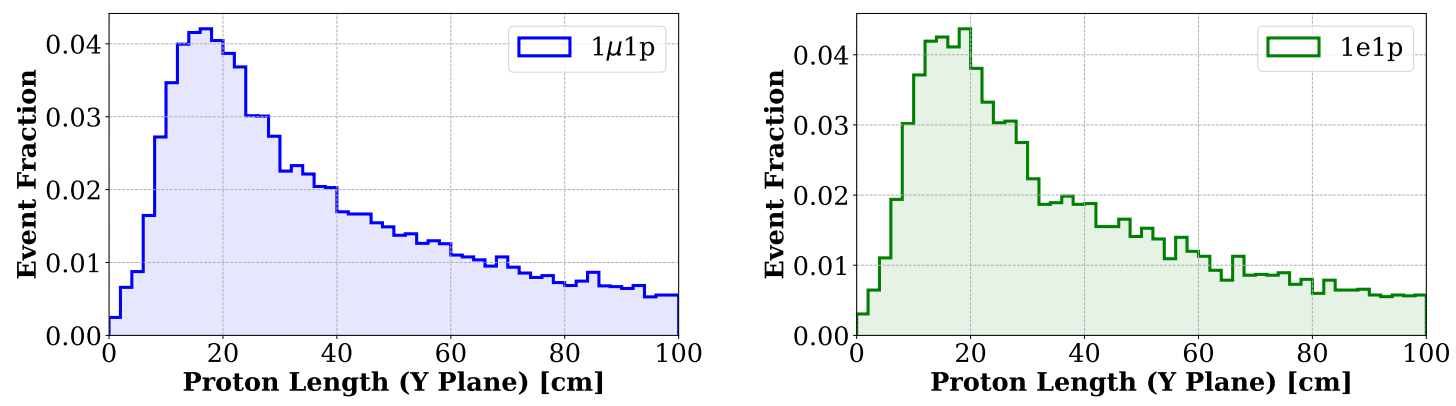

Figure 8.52: Proton length distribution for $1 \mu 1 \mathrm{P}$ (left) and $1 e 1 \mathrm{P}$ (right) events.
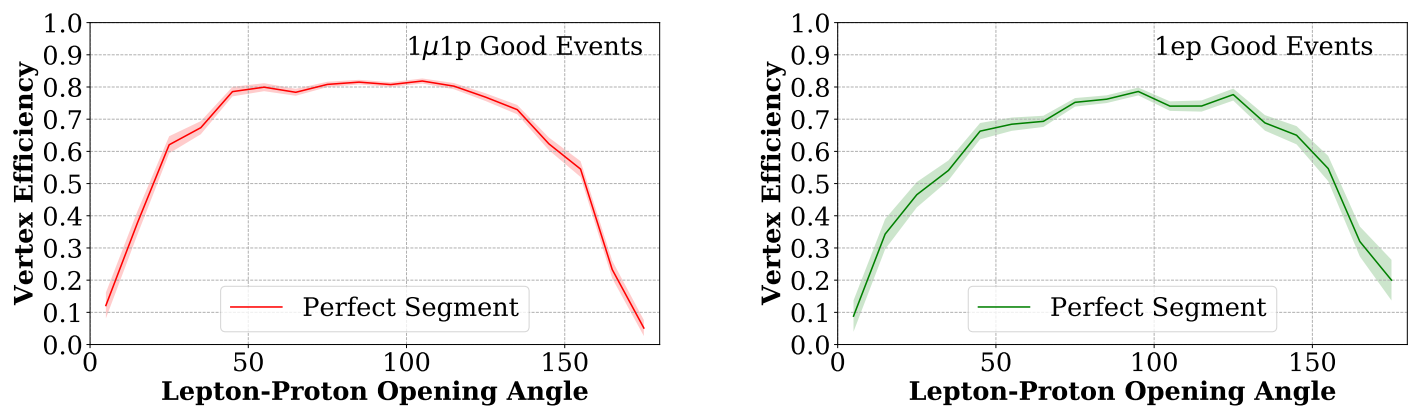

Figure 8.53: Vertex efficiency as a function of lepton and proton opening angle for $1 \mu 1 \mathrm{P}$ (left) and $1 e 1 \mathrm{P}$ events (right). The highest efficiency is achieved when the opening angle is near 90 degrees.

drops rapidly. This is due to particles being co-linear in the projected image and feature overlapping charge depositions. When the particle are perfectly co-linear, no vertex can be found. On the opposite end of the spectrum when the particles are emitted anti-parallel from the neutrino interaction point the efficiency decreases rapidly above 150 degrees. In this regime the vertexing algorithm is inefficient at locating obtuse vee shapes. The algorithm explicitly vetoes vertices which are located on vee shapes between 170 and 180 degrees opening angle. This helps reduce backgrounds associated with vertices found on cosmic rays. For both topologies, when the opening angle is within the range of 90 degrees, the vertex reconstruction efficiency approaches $80 \%$ or larger.

The vertex reconstruction efficiency naturally depends on the length of the emitted proton. In the low neutrino energy regime, the proton length is the topologically limiting 
factor for producing a vee shape. To study the effect of the proton length on the vertexing algorithm, the $3 \mathrm{D}$ proton length is projected onto the $\mathrm{Y}$ plane so the centimeter scale can be simply translated into pixel scale. The vertex reconstruction efficiency as a function of proton particle length is shown in Fig. 8.54
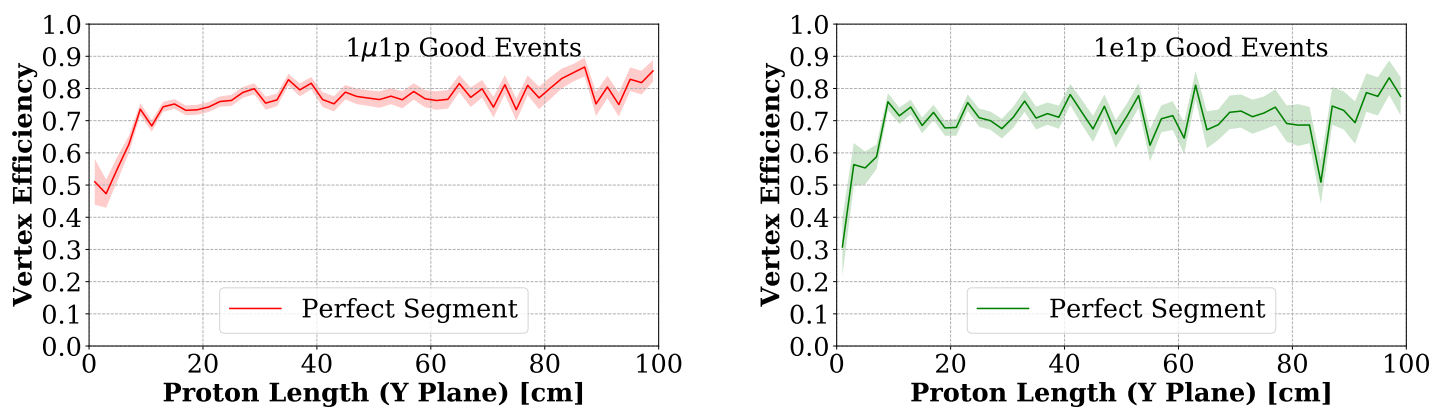

Figure 8.54: Vertexing efficiency as a function of proton length projected on to the $\mathrm{Y}$ plane image for $1 \mu 1 \mathrm{P}$ (left) and $1 e 1 \mathrm{P}$ (right) events. The efficiency decreases as the proton decreases in size.

Both topologies feature a similar efficiency profile as a function of proton length. An efficiency drop is observed when the proton length drops below $6 \mathrm{~cm}$. This threshold represents approximately the size of the largest circle used by the vertexing algorithm.

From studying the opening angle and proton length we can see the theoretical maximum vertexing efficiency for both $1 \mu 1 \mathrm{P}$ and $1 e 1 \mathrm{P}$ topologies approaches $80 \%$ or larger given perfect inputs. The vertex efficiency convolving all aspects of the upstream reconstruction and averaging over all kinematic variables is shown in Fig. 8.55

In the low energy region, the algorithm applied to the $1 \mu 1 \mathrm{P}$ sample is approximately $60 \%$ efficient while for the $1 e 1 \mathrm{P}$ sample is approximately $50 \%$. As the energy increases the algorithm efficiency on the $1 \mu 1 \mathrm{P}$ sample decreases in efficiency above $1 \mathrm{GeV}$, which can be attributed to the cosmic ray tagger, while the $1 e 1 \mathrm{P}$ sample remains relatively flat. A summary of the relative efficiency contributions for each of the three factors is shown in Table. 8.2 

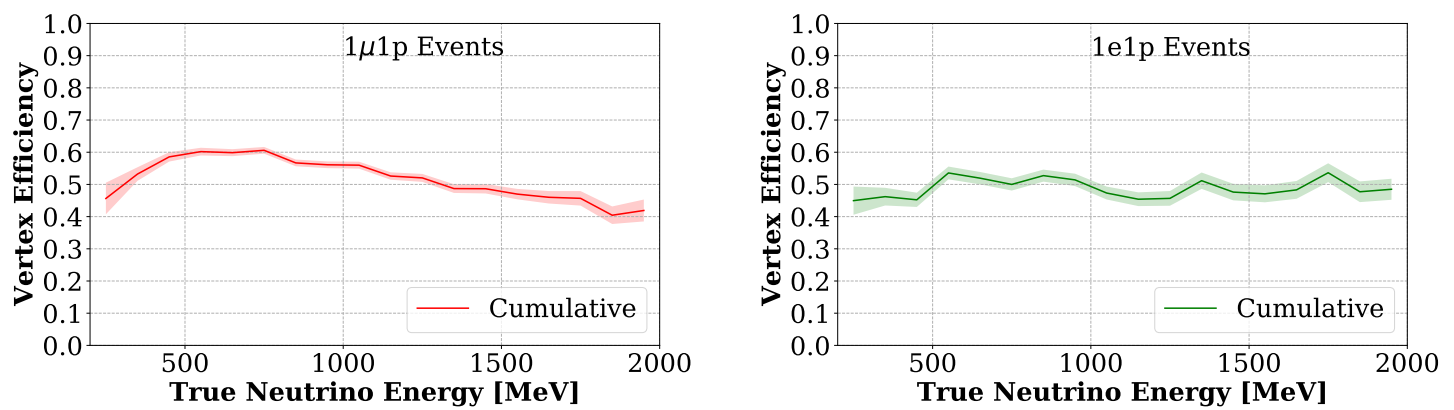

Figure 8.55: Cumulative vertexing efficiency as a function of true neutrino energy for $1 \mu 1 \mathrm{P}$ (left) and $1 e 1 \mathrm{P}$ (right) events.

\begin{tabular}{|l|c|c|}
\hline \multicolumn{1}{|c|}{ Factor } & $\begin{array}{c}\mathbf{1} \mu \mathbf{1 P} \text { Efficiency } \\
\text { (\% average) }\end{array}$ & $\begin{array}{c}\mathbf{1} e \mathbf{1 P} \text { Efficiency } \\
\text { (\% average) }\end{array}$ \\
\hline Good Event & 90 & 90 \\
\hline Vertexing & 80 & 70 \\
\hline SSNet & 95 & 90 \\
\hline Cosmic Tagger & 88 & 93 \\
\hline Overall: & $\mathbf{6 0}$ & $\mathbf{5 3}$ \\
\hline
\end{tabular}

Table 8.2: Table of relative efficiency for 5 components which contribute to the vertexing efficiency. The cumulative, or expected efficiency, for $1 \mu 1 \mathrm{P}$ and $1 e 1 \mathrm{P}$ events is shown in the bottom row.

\section{7 $1 \mu 1 \mathrm{P}$ Reconstruction}

\subsubsection{Track Finding}

The track finding algorithm takes as input the full LArCV ADC images for each of the three planes and the vertex 3D point. The ADC image is thresholded by zeroing all pixels of value less than 15 ADC. The tracking algorithm begins at the $3 \mathrm{D}$ vertex and does not use the output of the particle clustering algorithm due to its sensitivity to the presence of dead wires. Moreover, the location of the vertex within the cROI may cause the tracks and shower to exit that cROI. If this happens, although the vertex can be identified, the particle clustering may not reach the end of the tracks and showers, causing a mis-reconstruction. 
Additionally, it is important to have the capability to pursue a track beyond the cROI.

Reconstruction begins by finding a set of $3 \mathrm{D}$ points that belong to a given track by performing a stochastic search in the neighborhood of previously found 3D points. A regularization is then performed to find a minimal set of ordered 3D points that describe the track at the desired spatial resolution. Finally, observables such as length, local and average charge deposition, and angles are estimated.

Given a 3D point on a track (the vertex, or an already found 3D point), finding the neighboring points proceeds as follows:

- The seed of the reconstruction step is placed at the last found point.

- A set of random 3D points is generated inside a sphere of radius $r_{\text {search }}=2+4 * e^{-L / 5}$ $\mathrm{cm}$, where $L$ represents the distance between the vertex and the previous track point. The purpose is to allow a wider search around the vertex point. Once a track is found the restricted radius helps to prevent the track reconstruction from jumping to a nearby track.

- If the current track is longer than $5 \mathrm{~cm}, 3 \mathrm{D}$ points are chosen inside a forward going cone in the average direction of the track from the last $10 \mathrm{~cm}$. If the track is shorter than $10 \mathrm{~cm}, 3 \mathrm{D}$ points are chosen inside the cone of opening angle $30^{\circ}$. The forward search region extends to $2 \times r_{\text {search }}$. While the spherical search points allows for fine grained tracking and resolution of sudden direction changes, the forward search region allows faster progress on locally straight regions.

- Only points that project back on pixels with non-zero charge deposition on all planes are kept, with at most one plane on which the point projects on a dead wire.

- New points are added if the sum of the ADC values of the deposited charge on the pixels on which they project is greater than that of the already placed points of that iteration.

At this point, a set of neighbors to the seed are identified. Some of these points are not relevant, because they are too close to an already found point or track. Points are rejected is they are placed closer than $0.3 \mathrm{~cm}$ from an already existing point. All the remaining points 
at this stage are added to a proto-track, a cloud of un-related 3D points that correspond to non-zero pixels. The point within the new found points that is the furthest away from the current seed is now used as the new seed. The procedure is iterated as long as new points can be found ensuring the explored region pushes as far as possible along the track.

The points in the proto-track are not ordered and do not follow a linear path, zig-zagging back and forth within the track thickness. The next step orders the points by linking each one to its most likely neighbor. The next neighbor is found by minimizing a score ,

$$
\text { score }=5 \cdot L_{1}+0.1 \cdot L_{2}+2 \cdot(2-\cos \theta)-10 \cdot(2-\cos \phi) .
$$

The distances $L_{1}$ and $L_{2}$, as well as the angles $\theta$ and $\phi$ are summarized in Fig. 8.56a.

Once the points in the proto-track have been ordered, there is a logical path from one point to the next, and some points are rejected as they are not the best candidates according to the score function. A second pass through the track smooths the path and makes the more direct based on several criteria:

- a new set of points is created by performing a rolling average of two consecutive points,

- the new set is ordered by moving to the closest neighbor,

- the new point must be closer to the end point than the previous one,

- the distance from previous point cannot be more than $5 \mathrm{~cm}$, indicating a possible jump to another nearby track,

- the points that deviate by less than $0.5 \mathrm{~cm}$ from the line between points $\mathrm{n}-1$ and $\mathrm{n}+1$ are removed.

These operations are then iterated as long as a new track is found. An example of a reconstructed $1 \mu 1 \mathrm{P}$ event is shown in Fig. 8.7. Two tracks are found, corresponding to the trajectory of a muon and a proton. The tracking algorithm is able to follow both tracks to their true end points inside the detector as well as cross two regions of dead wires. 

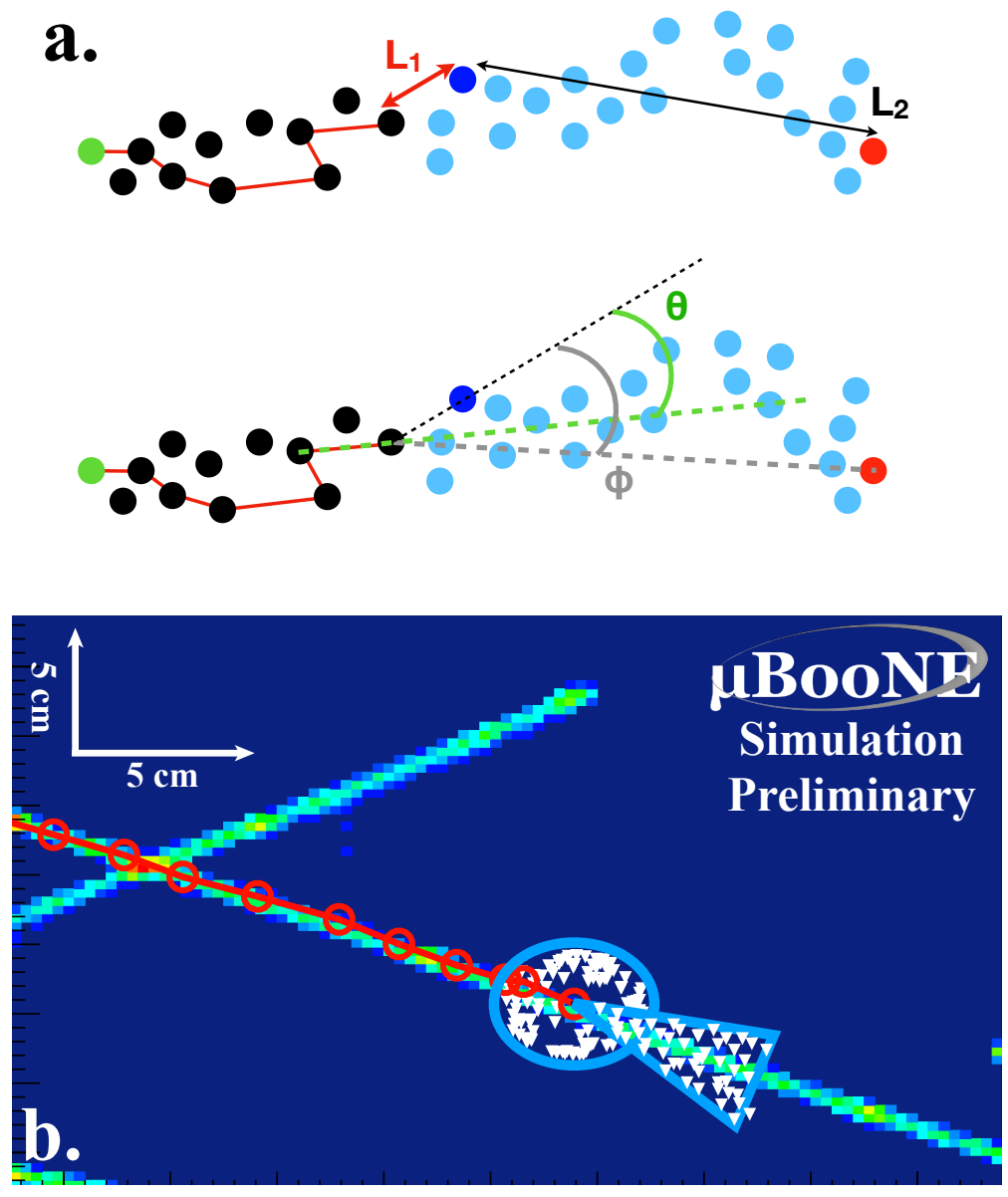

Figure 8.56: (a) Dots represent the set of 3D points in the proto-track. The black dots are the points that have been already sorted, and the light blue dots are the remaining unsorted points. The green and red dots correspond to the vertex and end of track respectively. The end point is selected as the 3D point the furthest away from the vertex. The red line corresponds to the path found within the sorted 3D points. For each unsorted point (dark blue point) the two lengths and angles are computed : $L_{1}$ is the distance to the last selected $3 \mathrm{D}$ point, $L_{2}$ is the distance to the end of the proto-track, $\theta$ is the angle from the last two sorted points to the candidate, and $\phi$ is the angle between the candidate, the last sorted point, and the end of the proto-track. (b) Tracking algorithm following a track using the stochastic search procedure. 


\subsubsection{Track Efficiency}

The efficiency of the track finding with respect to the kinetic energies of the individual particles at the vertex is shown in Fig. 8.57. The track efficiency is defined as the ratio of events with at least 1 track matched to the MC particle divided by the number of events. A track is considered reconstructed if the tracking algorithm follows a particle longer than $5 \mathrm{~cm}$ and ends near a particle's true end point.
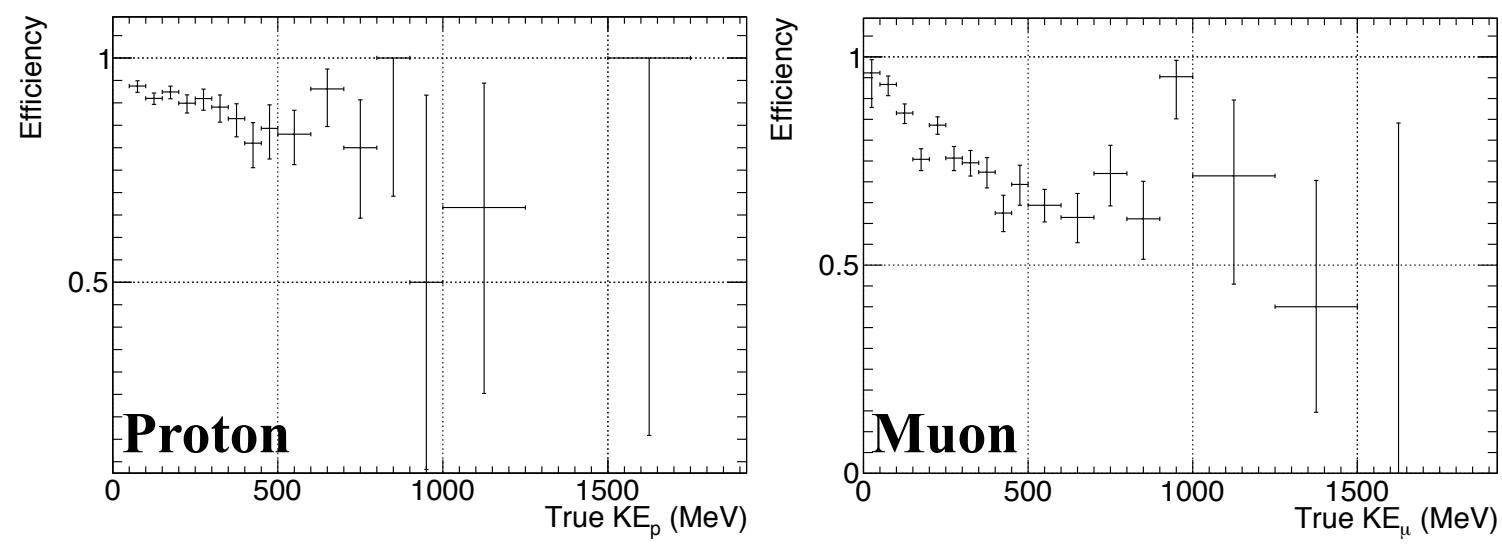

Figure 8.57: Tracking efficiency as a function of kinetic energy for proton (left) and muon (right).

Both efficiencies display a downward trend as the kinetic energy increases which is linked to the increased probability of encountering a failure as the track length increases. Fig. 8.58 shows the evolution of the tracking efficiency with respect to the true neutrino energy. The average efficiency of the track reconstruction is $(75.5 \pm 0.9) \%$ over the full energy range, however, a downward trend is visible, compatible with the behavior observed in the muon and proton single-particle efficiencies from Fig. 8.57. The efficiency within the energy range of interest to a low energy analysis $[200-500] \mathrm{MeV}$ is $(80 \pm 1) \%$

\subsubsection{Angular Resolution}

The track angle is estimated from the first $15 \mathrm{~cm}$ of the track near the vertex. The vector from the vertex to mean track position describes the path of the particle at short range. The angles $\phi$, projected angle in the $(\mathrm{X}, \mathrm{Y})$ plane, and $\theta$, angle with respect to the beam 


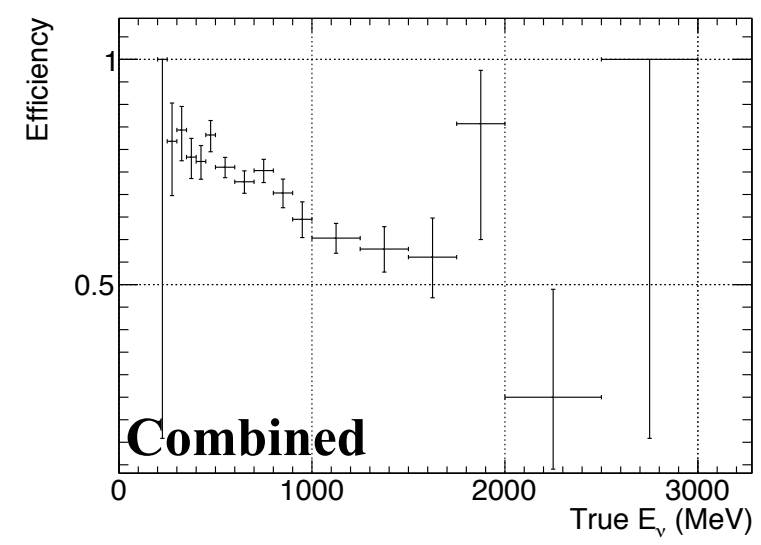

Figure 8.58: Tracking efficiency as a function of true neutrino energy for combined proton and muon track reconstruction.

axis, are evaluated for each track as described in Fig. 8.59.

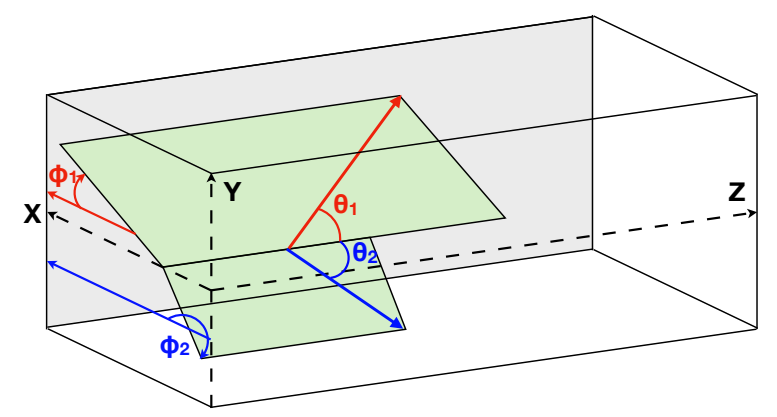

Figure 8.59: Track angle definition for $\phi$ and $\theta$ with respect to the MicroBooNE detector geometry.

Fig. 8.60 and Fig. 8.61 show the angular errors in the track reconstruction of protons and muons in $1 \mu 1 \mathrm{P}$ interactions.

\subsubsection{Energy Reconstruction}

The reconstruction of $3 \mathrm{D}$ tracks is used to obtain the track energy for the $1 \mu 1 \mathrm{P}$ topology. The $3 \mathrm{D}$ length of the track is computed by summing the distance between each consecutive 

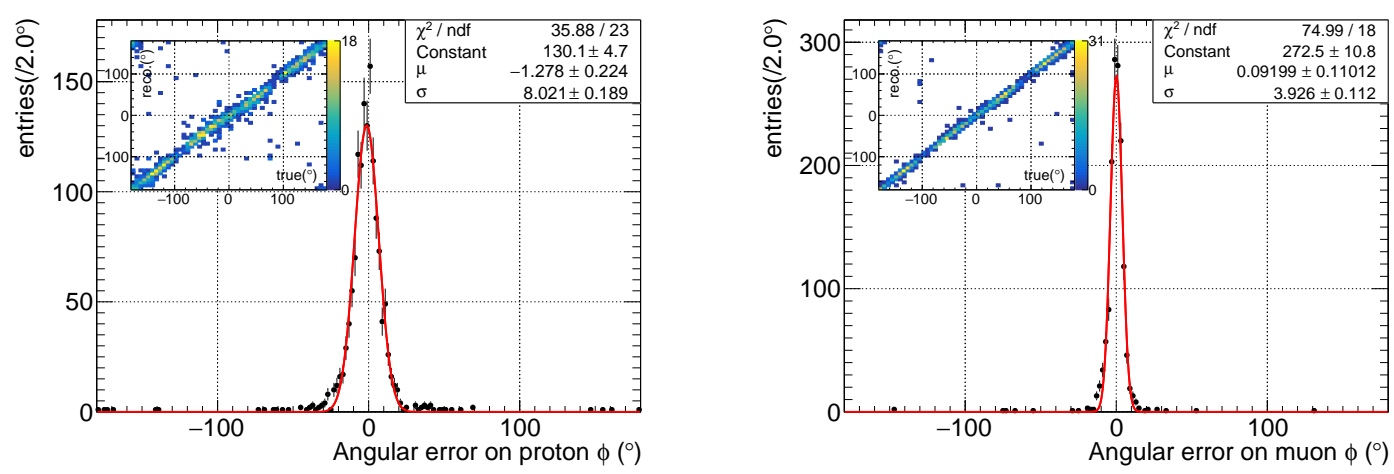

Figure 8.60: Error on the reconstruction of $\phi$ for protons (left) and muons (right). The red line corresponds to a Gaussian fit of the central peak. A resolution of (7.9 \pm 0.2$)$ deg. for protons and $(4.1 \pm 0.1) \mathrm{deg}$. for muons is achieved. The inserts in the upper left hand portion of the plot show the track by track correlation between the reconstructed and the true $\phi$ angle.
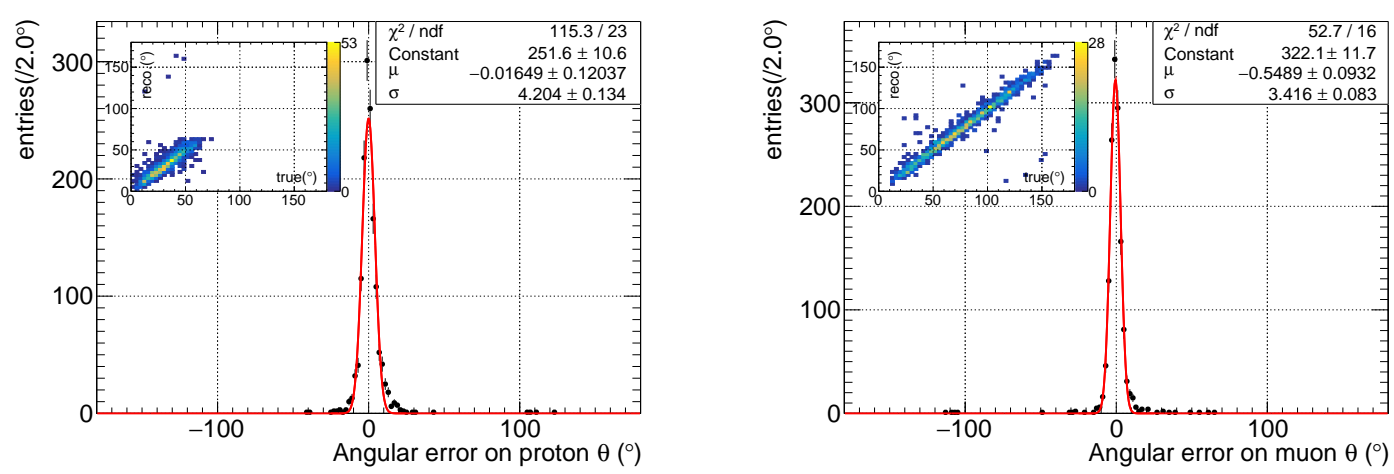

Figure 8.61: Error on the reconstruction of $\theta$ for protons left) and muons (right). The red line corresponds to a Gaussian fit of the central peak. A resolution of $(4.2 \pm 0.1)$ deg. for protons and $(3.5 \pm 0.1)$ deg. for muons is achieved. The inserts in the upper right hand portion of the plot show the track by track correlation between the reconstructed and the true $\theta$ angle.

point along the track. The length is then converted to kinetic energy using the known stopping power of each type of particle in liquid argon. The relationship between a proton 
and muons range and its kinetic energy is shown in Fig. 8.62 obtained from [39].

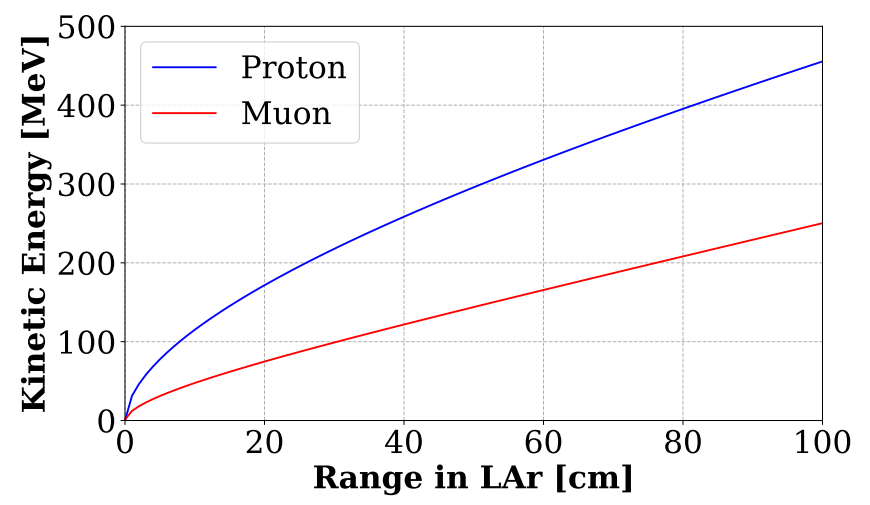

Figure 8.62: Kinetic energy as a function of range in liquid argon (LAr) for proton (blue) and muon (red) particles.

Fig. 8.63 shows the relative error made when reconstructing the energy of one of muon and proton tracks in $1 \mu 1 \mathrm{P}$ events as a function of the true particle kinetic energy using the range table lookup. The track is attributed to a proton or muon using MC truth information.
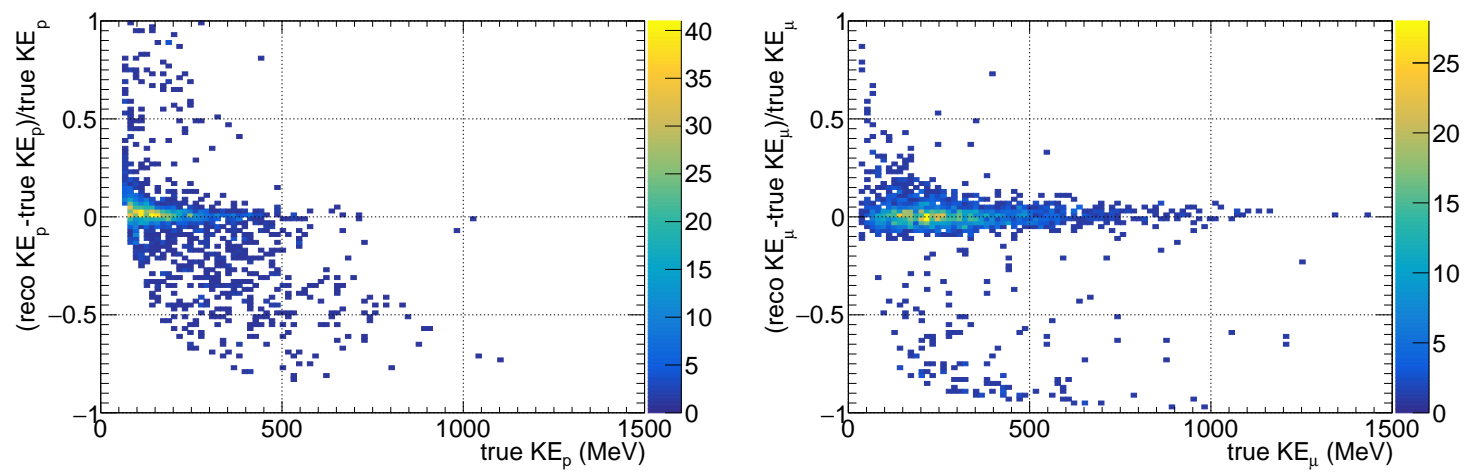

Figure 8.63: Relative difference between the reconstructed energy and the true energy as a function of the true kinetic energy at the single-track level proton (left) and muon (right) tracks.

The main populations are flat with a relative error centered on $\left(\mathrm{KE}^{\mathrm{reco}}-\mathrm{KE}^{\text {true }}\right) / \mathrm{KE}^{\text {true }}=$ 0 showing no systematic bias. 
To estimate the full energy of the neutrino, a range based definition is used,

$$
\mathrm{E}_{\nu}^{\text {range }}=\mathrm{KE}_{p}+\mathrm{KE}_{\mu}+m_{\mu}+m_{p}+B-m_{n}
$$

where $B \sim 40 \mathrm{MeV}$ is an effective nuclear binding energy [40]. Fig. 8.64 shows a comparison of the true and reconstructed energy. Each slice in true energy is fitted by a gaussian around its mean value. The fit results are shown as the black dots, and the errors on these dots correspond to the $\sigma$ of the Gaussian. A linear fit performed on the result shows a good linearity, with a slope factor of $0.97 \pm 0.01$ and an offset of $(19 \pm 6) \mathrm{MeV}$, for a neutrino energy range of $[200-1000] \mathrm{MeV}$.
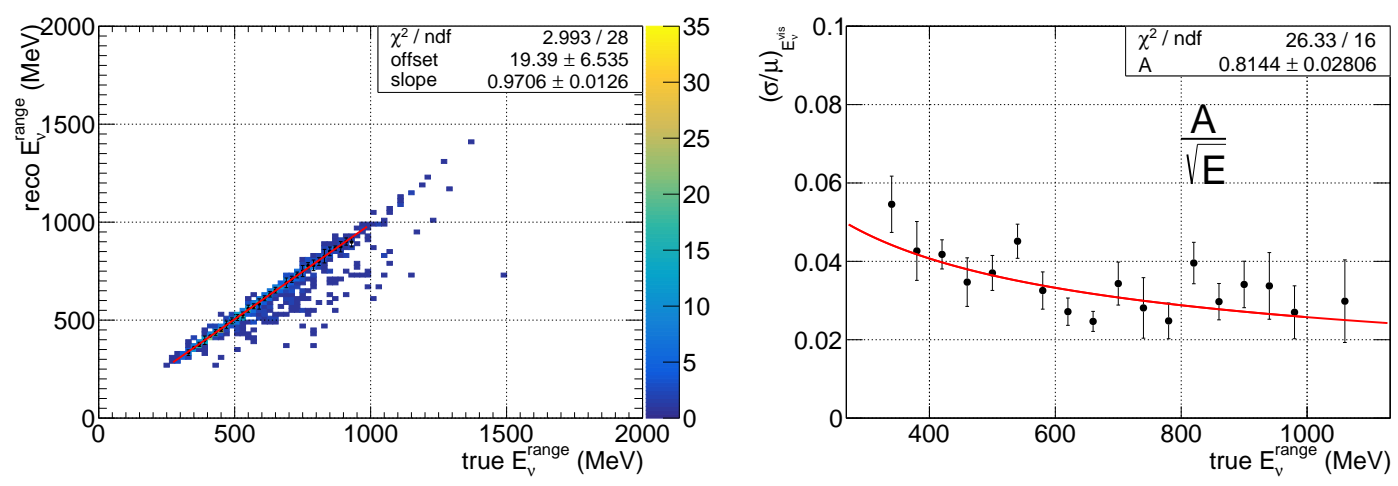

Figure 8.64: Left: Comparison of the reconstructed energy to the true MC energy. Right: Evolution of the resolution as a function of the true energy.

Fig. 8.64 also shows the evolution of the fractional resolution $(\sigma / \mu$ from the previous Gaussian fits) as a function of energy. The errors are the plot are errors on the parameters estimated by the Gaussian fit. The relative resolution follows a stochastically dominated reconstruction in $1 / \sqrt{E}$.

\subsection{5 $\mathrm{dE} / \mathrm{dx}$ Reconstruction}

For each $3 \mathrm{D}$ point, the local charge deposition, $d Q / d x$, for a given plane is computed by integrating the values of non-zero pixels in a 2 pixel radius around the projection of the $3 \mathrm{D}$ point on that plane. 
Once the local charge deposition around each 3D point has been found for each of the three planes, other physics features for particle identification can be derived such as the average $d Q / d x$ :

$$
\left\langle\frac{d Q}{d x}\right\rangle=\frac{1}{n} \sum_{i<n}\left(\frac{d Q}{d x}\right)_{i}
$$

where $\mathrm{n}$ is the number of reconstructed 3D points and $\left(\frac{d Q}{d x}\right)_{i}$ the local ionization of a given point $i$ using only the collection plane information.

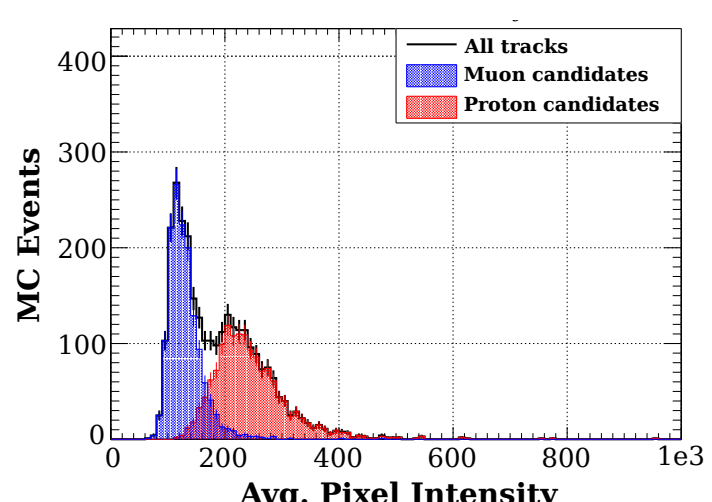

Figure 8.65: Average pixel intensity along each reconstructed track defined in Equation 8.5. The red and blue distributions represent the tracks with the highest (proton like) and lowest (muon like) average ionization.

The average ionization reconstructed for $1 \mu 1 \mathrm{P}$ simulated events in MicroBooNE is shown in Figure 8.65. Two underlying distributions are shown. The blue and red distributions are tracks matched to proton and muon particle respectively. The blue and red distributions can be separated by applying a cut on the average ionization as an example of particle identification at the track level.

Once the local charge deposition $d Q / d x$ is obtained, one can estimate the local deposited energy $d E / d x$ using the so-called modified box model [41] relationship,

$$
\frac{d E}{d x}=\frac{\rho \mathcal{E}}{\beta}\left[\exp \left(C \frac{d Q}{d x} \frac{\beta W_{\text {ion }}}{\rho \mathcal{E}}\right)-\alpha\right],
$$

where,

- $\rho=1.38$ is the density of the liquid argon, 
- $\mathcal{E}=0.273 \mathrm{kV} / \mathrm{cm}$ is the electric field, and

- $W_{\text {ion }}=23.6 \times 10^{-6} \mathrm{MeV}$ is the ionization energy.

The parameters $\alpha$ and $\beta$ are detector-independent and are expected with the values,

$$
\begin{aligned}
& \alpha=0.93 \pm 0.02 \\
& \beta=0.212 \pm 0.002(\mathrm{kV} / \mathrm{cm})\left(\mathrm{g} / \mathrm{cm}^{2}\right) .
\end{aligned}
$$

Finally, $C$ is a calibration factor accounting for the pixel ADC count to $e^{-} / \mathrm{cm}$.

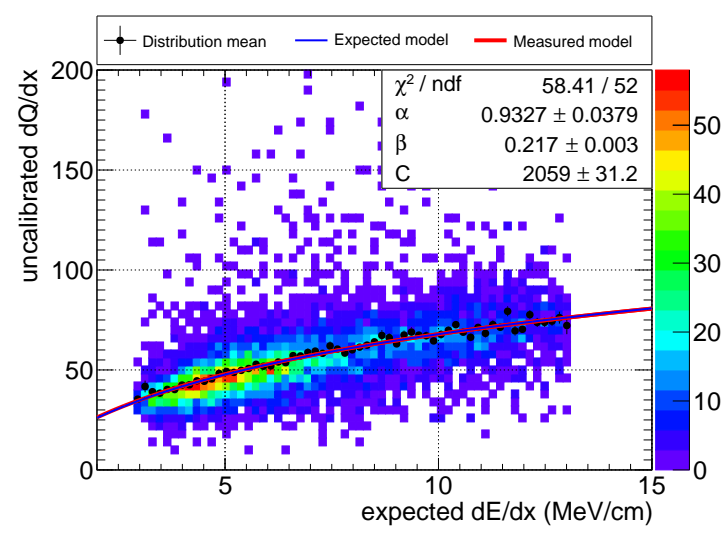

Figure 8.66: Estimation of the recombination parameters for the modified box model. The 2D histogram shows for each reconstructed 3D point over a sample of proton tracks, the pixel intensity as a function of the expected $d E / d x$. The black dots correspond to the average of the distribution for each slice in expected $d E / d x$. The blue line represents the expected profile, and the red line is a fit of the model to the black dots.

Fig. 8.66 shows the relationship between the measured $d Q / d x$ along the reconstructed proton candidate tracks and the expected $d E / d x$. For each point along the reconstructed tracks, the measured $d Q / d x$ is shown as a function of the expected $d E / d x$, estimated based on the residual length of the point (i.e. the distance along the track between that point and the end of the track).

The black dots correspond to the mean value in $d Q / d x$ for each vertical slice in $d E / d x$, with the error bar corresponding to the uncertainty on the mean position. The blue solid line corresponds to the expected relationship based on Equation 8.6 and the $\alpha$ and $\beta$ parameters 
from [41]. The red line corresponds to a fit of Equation 8.6 to the mean of the distribution. The parameters $\alpha, \beta$ and the calibration scale parameter are extract from simulated protons and found to be,

$$
\begin{aligned}
& \alpha=0.93 \pm 0.04 \\
& \beta=0.217 \pm 0.003(\mathrm{kV} / \mathrm{cm})\left(\mathrm{g} / \mathrm{cm}^{2}\right) \\
& C=2059 \pm 31\left(e^{-} / \mathrm{cm}\right) / \text { pixel }
\end{aligned}
$$

Using these parameters the $d E / d x$ at each reconstructed point can be converted from $d Q / d x$ along tracks. Figure 8.67 shows the $d E / d x$ profile as a function of the residual length for protons candidates, and for the corresponding muon candidates. Overlaid are the expected curves from [39].
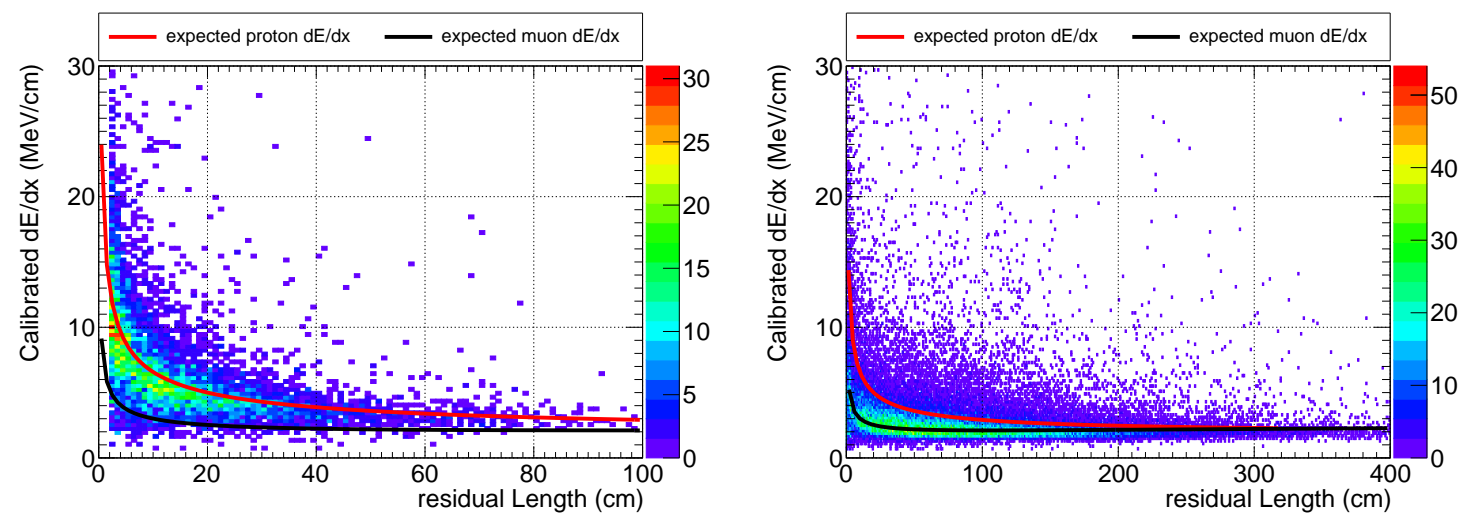

Figure 8.67: Distributions of reconstructed $d E / d x$ as a function of residual length for proton (left) and muon (right) tracks. The black and red lines respectively show the expected muon and proton $d E / d x$ versus residual length curves.

To correct for detector non-uniformities in charge collection a $d Q / d x$ correction factor is estimated using cosmic ray muon tracks. The LArTPC response is not uniform due to many factors: gain variation, dead wires, shorted wires, space charge effects, and attenuation due to impurities. After removing these effects at a low level, the cosmic ray muons can be used to remove residual non-uniformity. In this study we estimate a global $d Q / d x$ calibration by mapping the Y-Z spatial uniformity in charge collection. First, two to three meters long muon tracks are reconstructed using externally triggered cosmic ray data and a $d Q / d x$ value 
is calculated in a segmented location along the track. Next, the Y-Z plane is divided into cells. The correction factor per cell is calculated as the average $d Q / d x$ per cell divided by the global average of $d Q / d x$. The result is shown in Fig. 8.68.

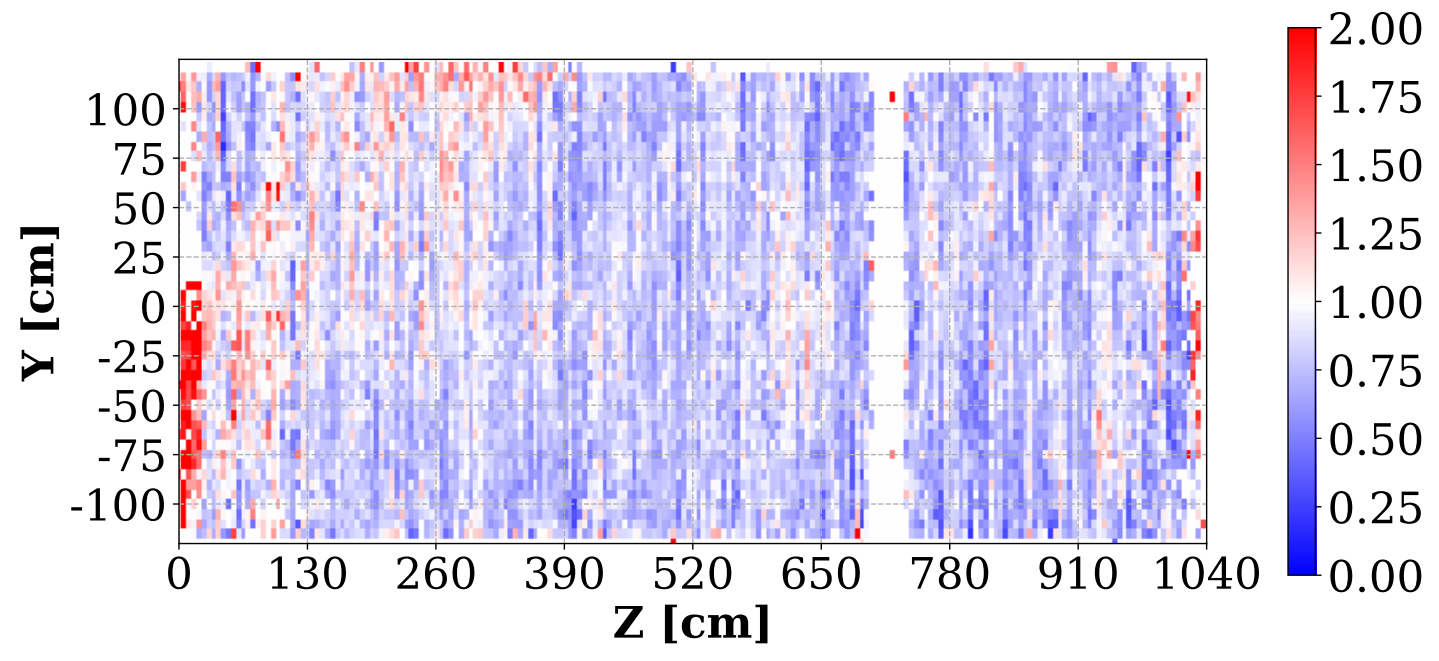

Figure 8.68: Correction factor as a function of the Y-Z detector space defined as the ratio of $d Q / d x$ in each divided by the global average $d Q / d x$.

Most cells feature an average calibration constant less than 1. A band of calibration factor larger than one can be observed in the low $\mathrm{Z}$ region of the detector. This region is associated with poor charge collection due to the presence of dead or noisy wires. An average calibration factor of approximately 0.8 is found over the $\mathrm{Y}-\mathrm{Z}$ space.

\subsection{1e1P Reconstruction}

\subsubsection{Topological Features}

Low energy $1 e 1 \mathrm{P}$ neutrino events feature two particles of distinct topological character connected at a single point in 3D space. In this algorithm the reconstruction and analysis are restricted to a true neutrino energy range below $600 \mathrm{MeV}$, the energy range of the predicted MiniBooNE low energy excess. In this energy regime the proton and electron particle each have simple topological features which can be used to separate signal from 
background.

The electron particle exhibits two distinct feature as it exits the neutrino interaction vertex as an electromagnetic shower. First is the presence of a tree-like structure characterized by a straight ionization pattern, called a trunk, near the vertex point and then evolving to a branching pattern. Second is the emission of electromagnetic radiation above a "critical" kinetic energy. Two effects contribute to the stopping power for electrons and determine it's energy loss in liquid argon as shown in Fig. 8.69.

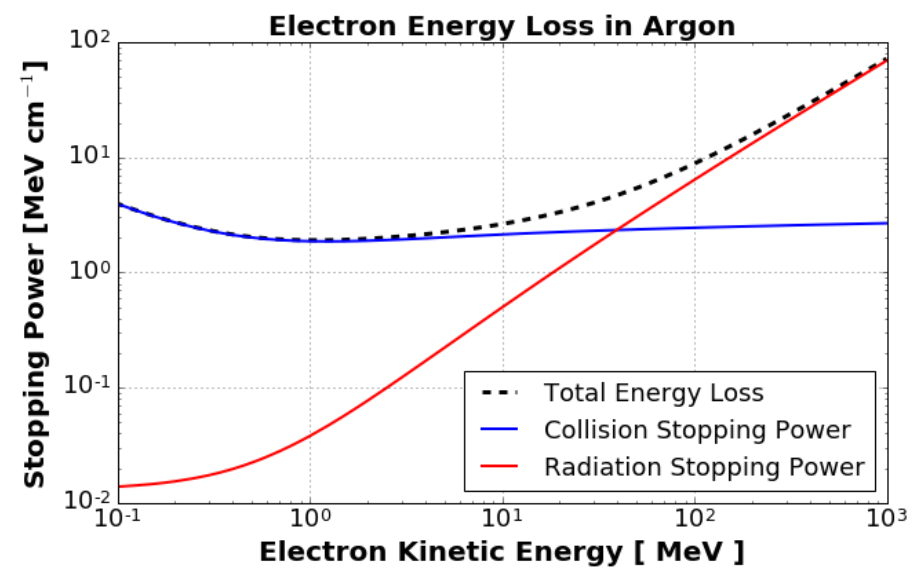

Figure 8.69: Energy loss for electrons as a function of initial kinetic energy.

The first effect is electron collisions with electrons located in the argon orbital cloud as the electron propagates through the bulk. This effect dominates the electron stopping power below approximately $30 \mathrm{MeV}$ kinetic energy. Above this so called critical energy, the electron will produce radiation in the form of photons. This radiation is called bremsstrahlung and produces a topological pattern in liquid argon. This patten involves an empty (charge-less) gap between the photon emission point, and where the photon converts into an electronpositron pair where a electromagnetic cascade begin to develop.

The electron deposited energy spectrum associated with low energy $1 e 1 \mathrm{P}$ neutrinos is shown in Fig. 8.70. The energy spectrum peaks at approximately $200 \mathrm{MeV}$ and extends up to approximately $500 \mathrm{MeV}$. In select true shower deposited energy bins, an event display from the Y plane is shown below the energy spectrum. As the shower energy is increased, the electromagnetic shower features described above develops. First, the electron shower grows 
in physical extent and contains an increasing number of prongs as well as becoming more tree like. Second, the electron begins to emit increasingly large electromagnetic radiation depositions.
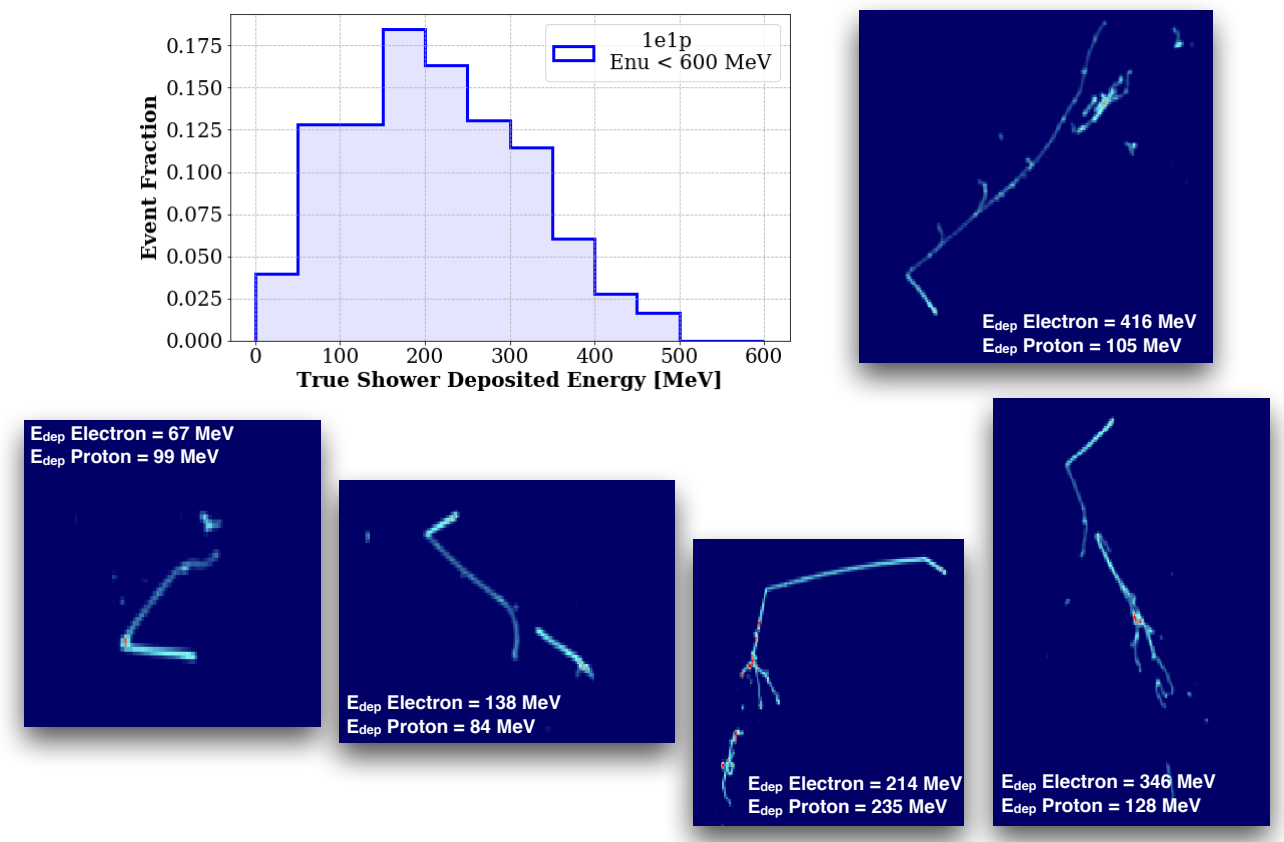

Figure 8.70: True shower deposited energy distribution for $<600 \mathrm{MeV} 1 e 1 \mathrm{P}$ interactions. Event displays from five energy bins are shown to illustrate the electron features described in Section 8.8.1.

The lowest energy bin in Fig. 8.70, which contains events both above and below the electron critical energy for radiation, represents approximately $5 \%$ of interactions. The feature presence of a tree like structure, and presence of radiation emitted in the forward direction of the electron are targeted in reconstruction to identify an electron particle.

The proton particle's ionization pattern for low energy 1e1P interactions is characterized by a straight line with increasing charge deposition toward the end of the track. Shown in Fig. 8.71 is the proton deposited energy for $1 e 1 \mathrm{P}$ events below $600 \mathrm{MeV}$. The energy 
distribution peaks in the second bin of 50 to $100 \mathrm{MeV}$ deposited charge and falls off to 300 $\mathrm{MeV}$. In three energy bins an event display shows a proton particle's line-like topological structure. As the proton particle increases in deposited energy, the proton track increases in length while remaining straight. As the proton travels outward from the vertex an increase in the ionization per unit length can be seen. Both the line like structure and the $d E / d x$ features are targeted in algorithm to identify a proton particle.
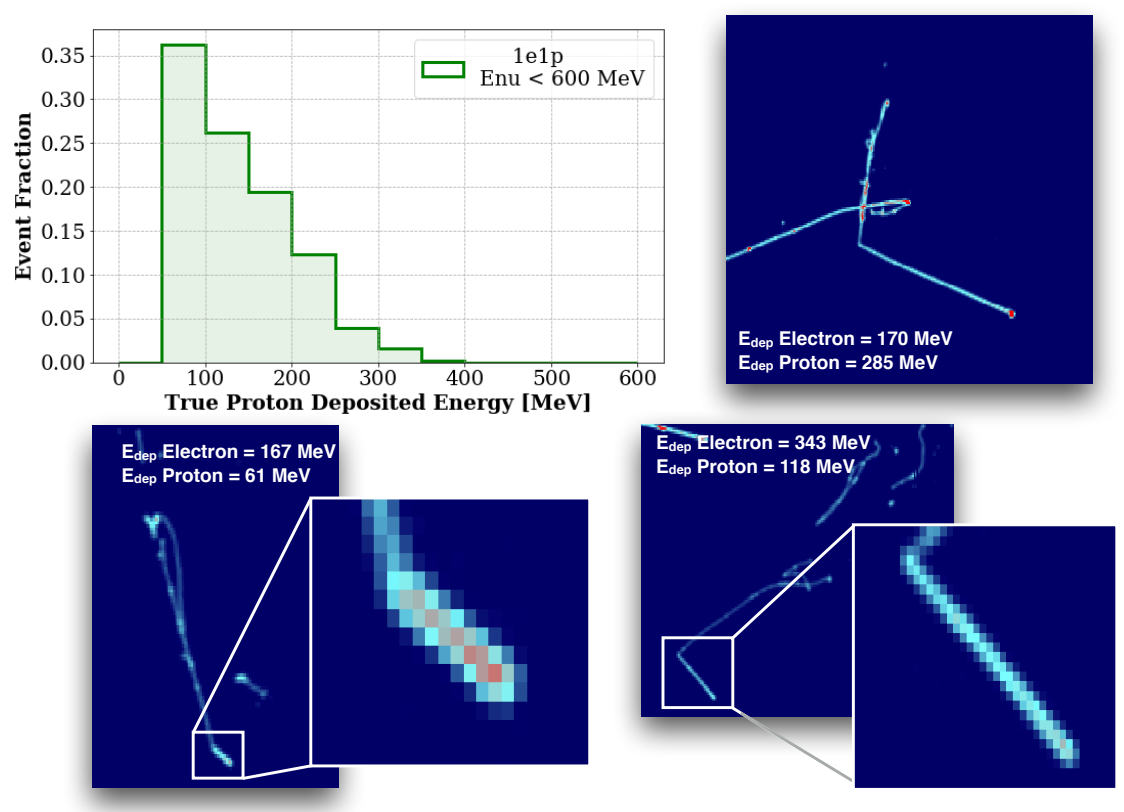

Figure 8.71: True proton deposited energy distribution for $<600 \mathrm{MeV} 1 e 1 \mathrm{P}$ interactions. Event displays from three energy bins are shown to illustrate the proton features described in Section 8.8.1.

Finally, the $1 e 1 \mathrm{P}$ algorithm provides improved particle clustering capability by alleviating some inefficiencies in the generic clustering algorithm. The particle clustering algorithm developed in the vertex finding code is incapable of associating disconnected charge to clustered particles. For shower type particles which typically feature the presence of 
bremsstrahlung, the disconnected charge will not be optimally clustered. Additionally, the particle clustering algorithm is heavily impacted by interference of the cosmic ray tagging algorithm described in Section 8.4.4. Performing topological analysis on a particle cluster which may have some component truncated by the cosmic ray tagging algorithm will not provide complete features. In the $1 e 1 \mathrm{P}$ algorithm the cosmic ray tagging is turned off.

\subsubsection{1e1P Algorithm}

The $1 e 1 \mathrm{P}$ algorithm is a LarOpenCV based traditional algorithm to reconstruct less than $600 \mathrm{MeV}$ neutrino events. By restricting our target neutrino energy range to below $600 \mathrm{MeV}$ the algorithm is applied in a restricted region around the $3 \mathrm{D}$ reconstructed vertex point. This region fully contains the ionization pattern associated with the electron and proton particle. The crop region around the vertex is set with an edge length of 120 centimeters (400 pixels) such that activity around the vertex is captured at least 60 centimeters (200 pixels) in each direction. A crop size of 60 centimeters has been optimized using a Monte Carlo truth level study of low energy $1 e 1 \mathrm{P}$ events as shown in the left plot of Fig. 8.72 .

The truth length distribution projected on the $\mathrm{Y}$ plane for proton and electrons is shown for sub $800 \mathrm{MeV} 1 e 1 \mathrm{P}$ events. Both the electron and proton particle complete their charge deposition within 200 pixels from their true vertex point. The right hand plot of Fig. 8.72 shows a low energy $1 e 1 \mathrm{P}$ event on the $\mathrm{Y}$ plane. A typical low energy $1 e 1 \mathrm{P}$ event features an electron of approximately 20 centimeters (70 pixels) and a proton of 3 centimeters (10 pixels).

The location of the $3 \mathrm{D}$ vertex is placed at the center of the image and provides the starting point for electron and proton feature finding. An example LArCV image input into the $1 e 1 \mathrm{P}$ algorithm is shown in Fig. 8.73 .

The image displays a $225 \mathrm{MeV} 1 e 1 \mathrm{P}$ neutrino which produces a single proton and electron as projected onto the three MicroBooNE TPC planes. Each plane image is a 60 centimeters square crop around the vertex and centered at the reconstructed vertex location. One electron of $174 \mathrm{MeV}$ is emitted producing a branch like structure and some amount of radiation. Connected to the electron is a $50 \mathrm{MeV}$ proton which appears bright red (indicating high charge deposition). Also present in the image are cosmic rays which 

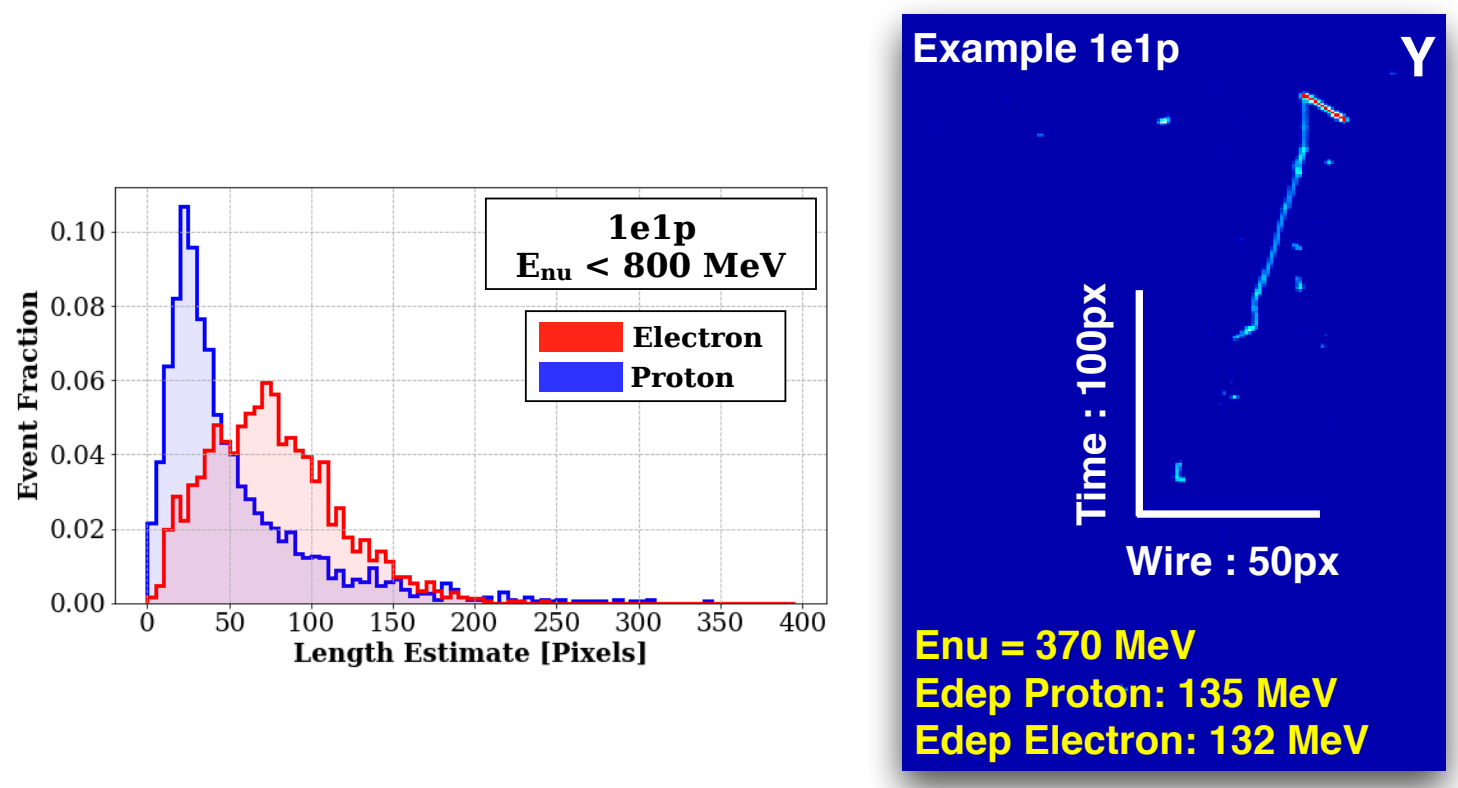

Figure 8.72: Left: Distribution of electron (red) and proton (blue) true particle length as projected onto the Y plane for sub $800 \mathrm{MeV} 1 e 1 \mathrm{P}$ interactions. Right: A $370 \mathrm{MeV} 1 e 1 \mathrm{P}$ interaction with X and Y pixel scale. The charge deposition fits in a 400 x 400 pixel window centered around the vertex.

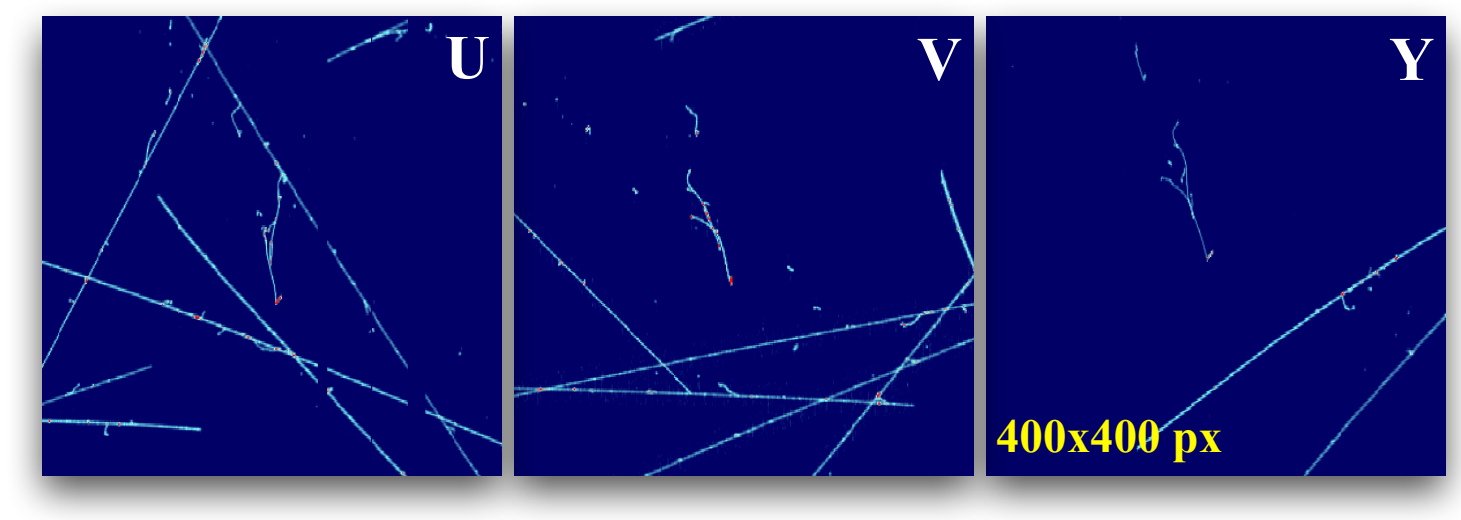

Figure 8.73: An example input into the $1 e 1 \mathrm{P}$ algorithm showing a $400 \times 400$ pixel crop around the vertex in each of the three planes.

can either pass through the image or enter the image and stop. In some cases, the cosmic ray will be fully contained in the image. Cosmic rays observed in these image crops are 
easy for the human eye to distinguish from electron neutrinos and a simple algorithm has been developed to remove them.

In a typical image crop, cosmic rays pass through from one edge to another, or enter the image edge and stop inside. An algorithm identifies cosmic rays using $2 \mathrm{D}$ information only to alleviate reconstruction inefficiencies associated with 3D track finding. The three steps for identifying cosmic rays are shown in Fig 8.74 .

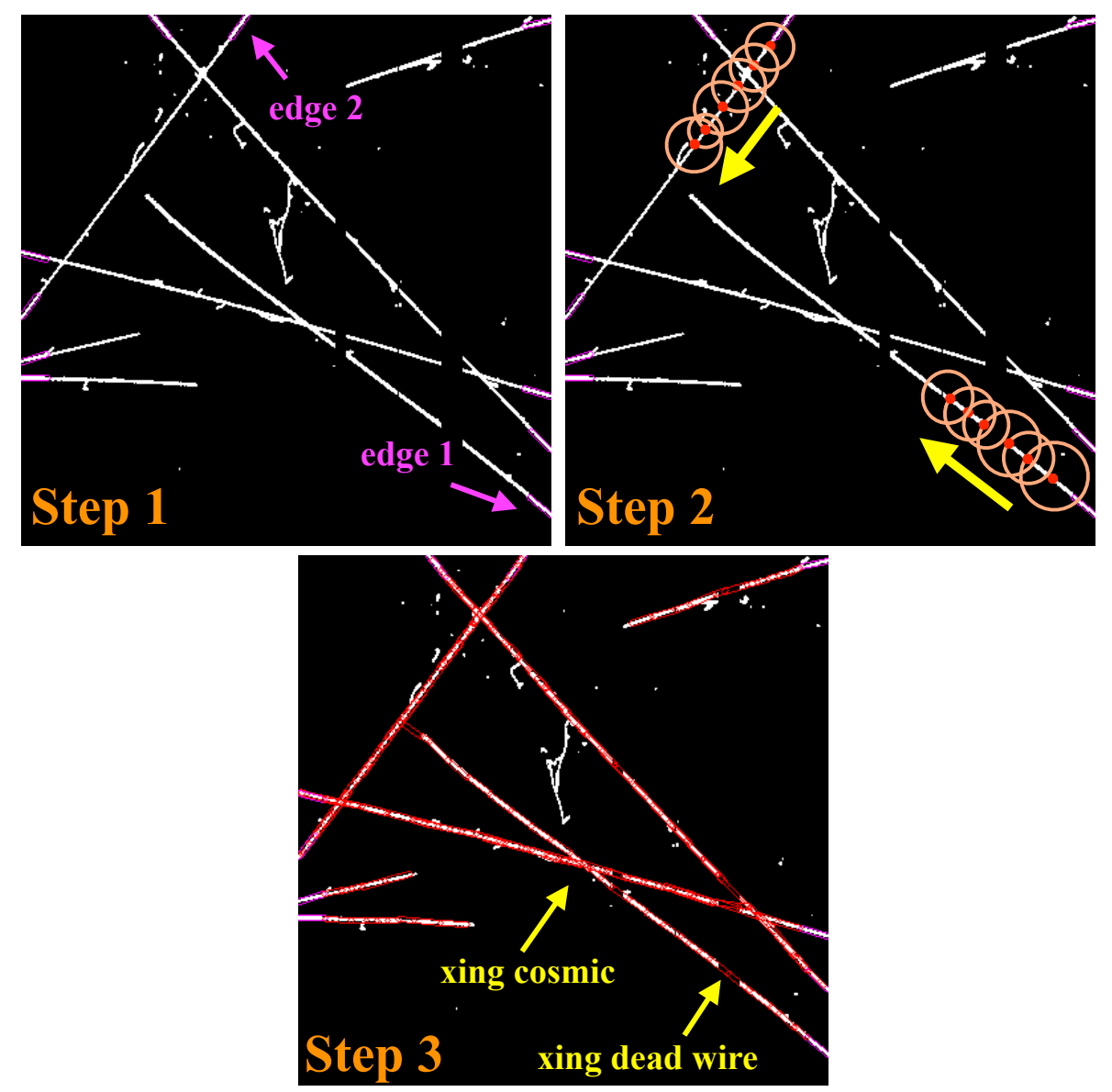

Figure 8.74: Steps for identifying cosmic ray tracks within the image crop described in Section 8.8 .2

First, edge crossings are identified on the image boundary by locating charge on the border as shown in Step 1. A 6 centimeter line is drawn on the image to best estimate the $2 \mathrm{D}$ direction of the line at the border. This is done by optimizing the number of pixels for which the $2 \mathrm{D}$ line covers in a half circle around the border point. The lines are 3 pixels 
thick and are shown in purple color. In the Step 1 a total of 10 edges are identified. Next, in Step 2, the lines are followed in 2D using a custom line following algorithm. Starting at the border point, the line is followed using a series of graduated circles. The algorithm moves from one point to the next by minimizing the distance between the previous circle crossing point and the next crossing point. The algorithm is always attempting to follow the straightest possible path but is allowed to bend gradually to handle the effect of cosmic rays curving due to the space charge effect. The algorithm can also cross dead wires by changing its circle size to step over the dead wire region or by using a custom algorithm to compute a linear extension for the cosmic ray track inside the dead regions. The full cosmic ray tagged result is shown in Step 3. The red lines are an output of the cosmic ray tagging algorithm's attempt to follow the cosmic ray from the image boundary. There are three features to note. First, the cosmic tagger can cross dead wires as indicated by the yellow arrow. Second, the tagger can cross other cosmic rays in the same plane. This is because the algorithm is trying to follow a straight line and is penalized for deviating from a straight trajectory. Third, the cosmic ray tagger does not tag the neutrino interaction in the upper right portion of the image. Here you see the red line pass by the electron particle as well as the associated electrons radiated photon. The red pixels are tagged, and removed from the image leaving only the neutrino interaction pixels and various delta rays attached to the tagged cosmic ray tracks. Examples of the cosmic ray tagger for reconstructed $1 e 1 \mathrm{P}$ events is shown in Fig 8.75 .

Once the cosmic rays have been tagged and removed from the image the algorithm reconstructs particle clusters using an optimized method for low energy $1 e 1 \mathrm{P}$ events as described in Fig. 8.76 .

Step 4 of Fig. 8.76 shows the result of removing tagged cosmic pixels from the crop, leaving only the neutrino interaction. Next, in Step 5, the pixels within 5 centimeters of the vertex are identified. Using a circle crossing point method, clusters are uniquely identified coming out of the vertex. A triangle shape is fit with floating base length and height using an oriented bounding box algorithm to enclose the cluster. The triangle serves as a generic shape to capture the physical extent of the particle in two dimensions and define the rough direction of propagation. In Step 6 the $2 \mathrm{D}$ direction of each particle is optimized 

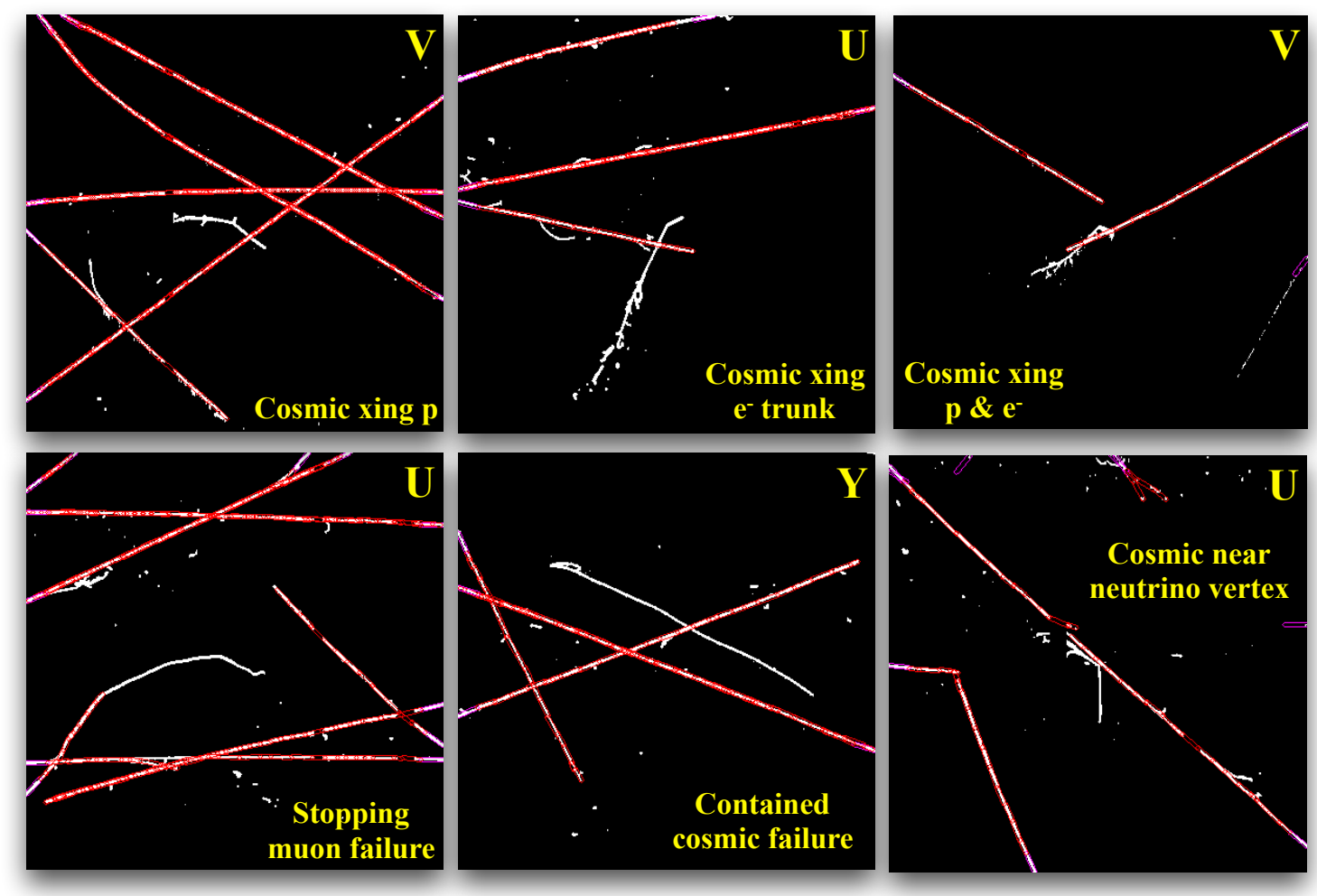

Figure 8.75: Examples of the cosmic ray tagging algorithm applied to six reconstructed vertices. Top Row: Cosmic algorithm crosses a proton particle of a $1 e 1 \mathrm{P}$ interaction. Cosmic algorithm crosses the trunk of an electron particle. Cosmic crosses both the electron and proton of a $1 e 1 \mathrm{P}$ interaction. Bottom Row: Cosmic algorithm follows a stopping muon until the track bends too severely, stopping before the true end point. A stopping muon is completely contained inside the image and not tagged by the cosmic algorithm. Cosmic tagger touches a $1 e 1 \mathrm{P}$ interaction vertex.

by perturbing the location of the $2 \mathrm{D}$ projected vertex (red dots) and drawing a line (cyan color) between the perturbed vertex and the base of the fit triangle. The $2 \mathrm{D}$ line is scanned inside the triangle to maximize the number of non-zero pixels enclosed inside. The result is a line which best represents the estimated $2 \mathrm{D}$ direction of the particle trajectory. The benefits for using a triangle and $2 \mathrm{D}$ line to represent the initial particle cluster rather than a generic cluster are two fold. First, it gives the particle a well defined temporal extent which 

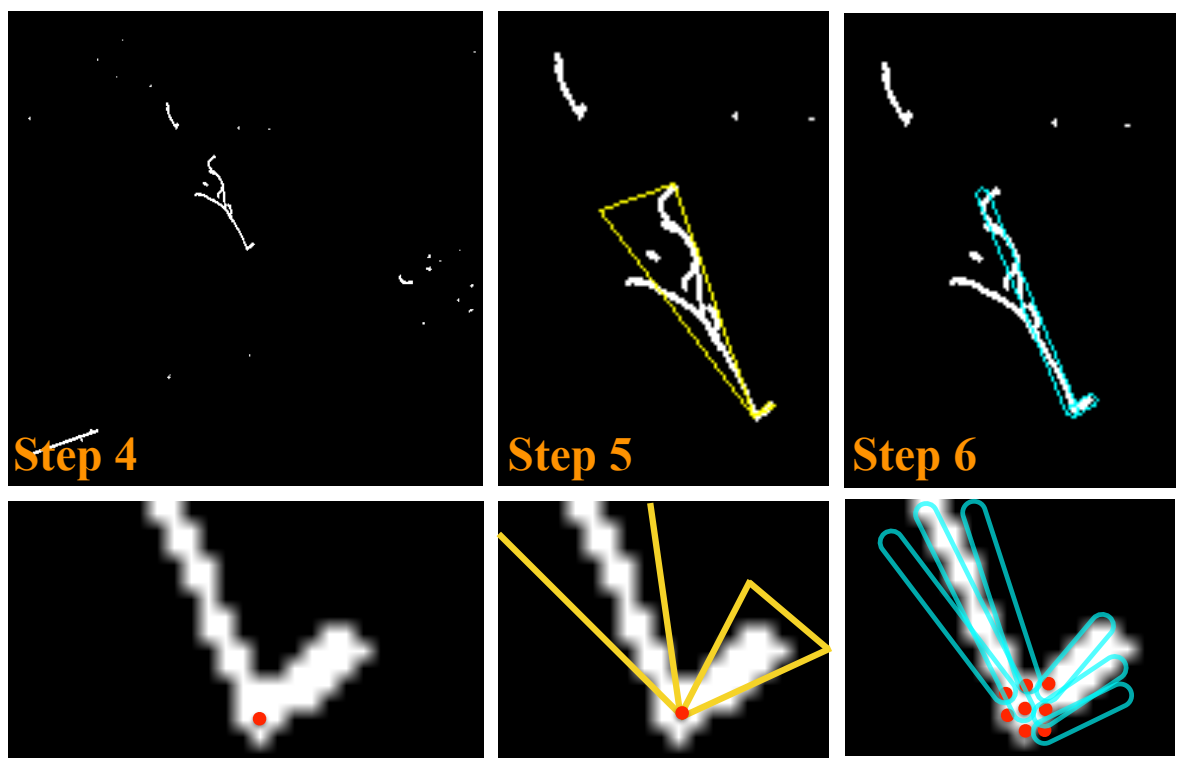

Figure 8.76: Steps 4-6 of the 1e1P algorithm as described in Section 8.8.2

improves the chance of matching across planes. Second, it allows the cluster to be connected across tagged cosmic rays and dead wire regions by recursively extending the line and refitting the bounding triangle until all charge associated with the particle is collected. With the ability to extend across these two regions, the maximum amount of connected charge can be identified as coming from the vertex point giving a better topological representation of the particle.

After the clusters have been identified on each plane, the 2D lines are matched across planes using the time overlap algorithm using the method described in Section 8.6.4. Particle 1 (P1) and particle 2 (P2), have been identified using the algorithm as shown in Fig. 8.77. $\mathrm{P} 1$ has been reconstructed using two planes of information, the $\mathrm{U}$ and $\mathrm{Y}$ plane. $\mathrm{P} 2$ has been reconstructed using 3 planes of information, the $\mathrm{U}, \mathrm{V}$ and $\mathrm{Y}$ planes.

\subsubsection{Topological Analysis}

A number of topological parameters are estimated for each reconstructed particle to identify the presence of an electron and proton particle. For all particles attached to the vertex the momentum is reconstructed. In addition, the $3 \mathrm{D}$ length and $d E / d x$ are characterized. 


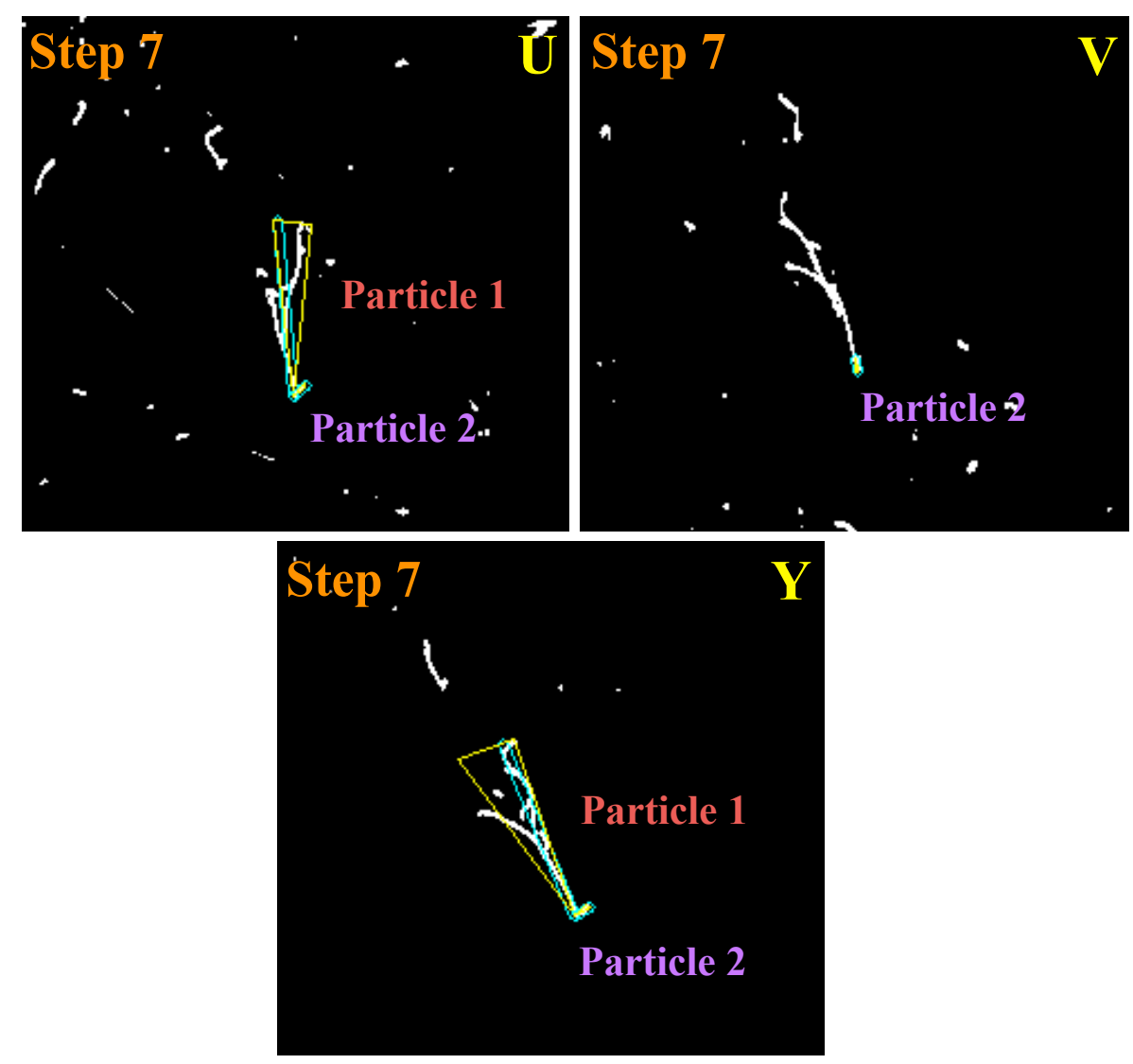

Figure 8.77: The final step of the $1 e 1 \mathrm{P}$ algorithm as described in Section 8.8.2. Two particles have been reconstructed and matched across planes.

To determine if an electron particle is present at the vertex the cluster shape is analyzed to detect features shown in Fig. 8.78 using a custom set of OpenCV based algorithms. The electron shower radiation is identified by extending a triangle in the $2 \mathrm{D}$ direction of the cluster whose length is a function of the radiation length and the length of the trunk as shown in Fig. 8.78a. Charge which is enclosed by this extended triangle is compared across planes to determine if the pixels are 3D consistent. Topological features such as the number of branch points and prongs extending off a common trunk are identified to characterize the charge deposition profile as the particle exits the vertex shown in Fig. 8.78c. In Fig. 8.78d,e] features such as determining the fraction of empty area inside the convex hull as well as the number, and length of the defect lines in the trunk cluster used to understand if a tree like structure is present. 
To determine if a proton particle is present at the vertex the cluster is simply compared with a straight line as shown in Fig $8.78 \mathrm{~b}$. A low energy proton particle exhibits a straight line trajectory in the LArTPC image as described in the previous section. To know if a straight line exists coming out of the vertex, a straight line of thickness 3 pixels is drawn from the $2 \mathrm{D}$ vertex location to the cluster end point. If the underlying particle cluster is consistent with the drawn straight line, in the sense of number of pixels covered by the line, then the particle is straight. Testing a straight line template is an economical way to determine if the cluster is straight and avoids a principle component analysis of the cluster.
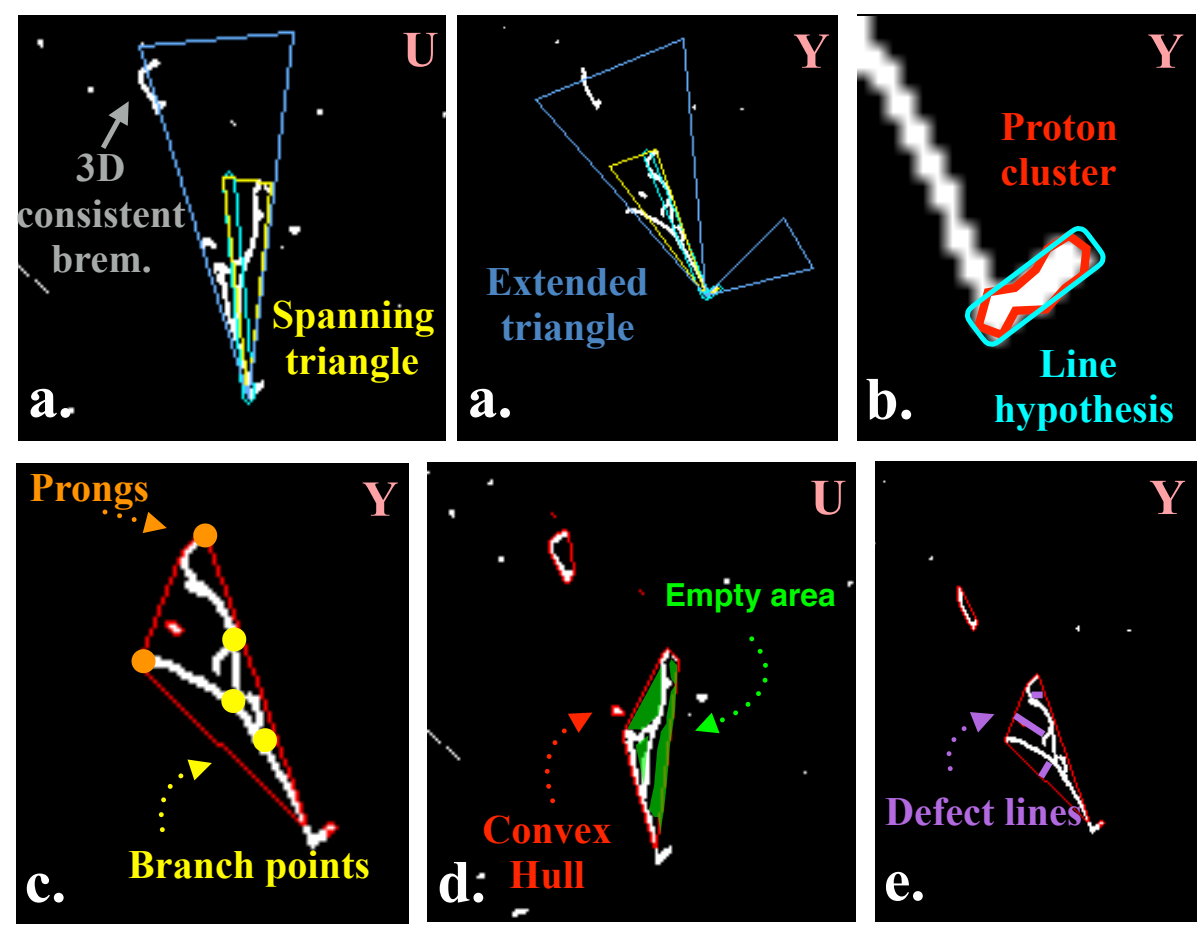

Figure 8.78: Topologically reconstructed parameters for electron and proton particles using the $1 e 1 \mathrm{P}$ algorithm described in Section 8.8 .3

\subsubsection{Angular Reconstruction}

The $3 \mathrm{D}$ direction of a shower is reconstructed by transforming the two $2 \mathrm{D}$ directions reconstructed for the pair of particles into $3 \mathrm{D}$ coordinates. The $2 \mathrm{D}$ direction on each plane is calculated by measuring the charge-weighted $2 \mathrm{D}$ direction with respect to the vertex 
location $\left(x_{\mathrm{vtx}}, y_{\mathrm{vtx}}\right)$ of all pixels associated with the particle on a given plane according to,

$$
\begin{aligned}
& x_{p}=\frac{1}{\sum_{i=0}^{N_{\text {pixel }}} Q_{i}} \times \sum_{i=0}^{N_{\text {pixel }}} Q_{i}\left(x_{i}-x_{\mathrm{vtx}}\right) \\
& y_{p}=\frac{1}{\sum_{i=0}^{N_{\text {pixel }}} Q_{i}} \times \sum_{i=0}^{N_{\text {pixel }}} Q_{i}\left(y_{i}-y_{\mathrm{vtx}}\right),
\end{aligned}
$$

where the components $x_{p}$ and $y_{p}$ represent the vector components of a particle $2 \mathrm{D}$ direction on plane $p$. Given two 2D weighted directions, the 3D direction is calculated using geometric relations between the planes and clusters. The reconstructed 3D angle of the particle with respect to the beam direction is a useful input into the CCQE formula which can be used to estimate the neutrino energy.

The ability to resolve the proton direction with respect to the beam is shown in Fig. 8.79 for $1 e 1 \mathrm{P}$ events with true neutrino energy less than $800 \mathrm{MeV}$. A magenta line at $y=x$ is used to guide the eye.
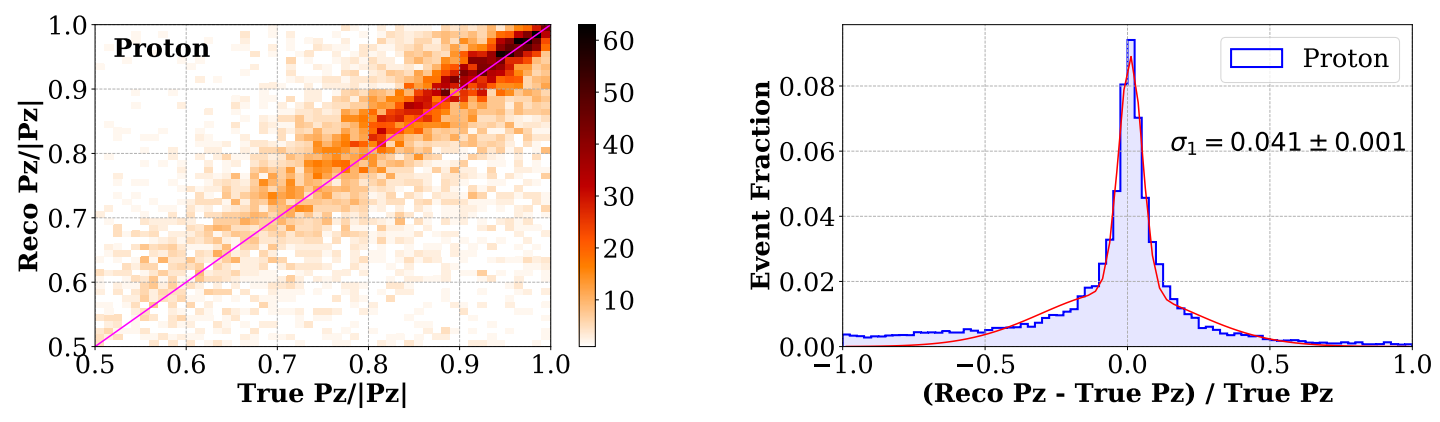

Figure 8.79: Right: Reconstructed versus true proton angle with respect to the beam for a sample of $1 e 1 \mathrm{P}$ events. The magenta line shows $y=x$ to characterize the bias. Left: Fractional difference between the reconstructed and true angle with a double gaussian fit. The angular resolution is approximately $4 \%$.

Proton particles produced in low energy $1 e 1 \mathrm{P}$ interactions are primarily forward going with respect to the beam. Good agreement is found between the reconstructed proton angle with respect to the beam versus true angle. The fractional resolution of the proton angle is approximately $4 \%$ as estimated from a double gaussian fit to the fractional difference between the reconstructed and true angle. The fractional angular resolution increases as the 
proton kinetic energy decreases since the proton particle spans less pixels, as reconstructing the proton direction becomes challenging.

The ability to resolve the electron direction with respect to the beam is shown in Fig. 8.80. A good agreement is found between the reconstructed electron direction and the true direction.
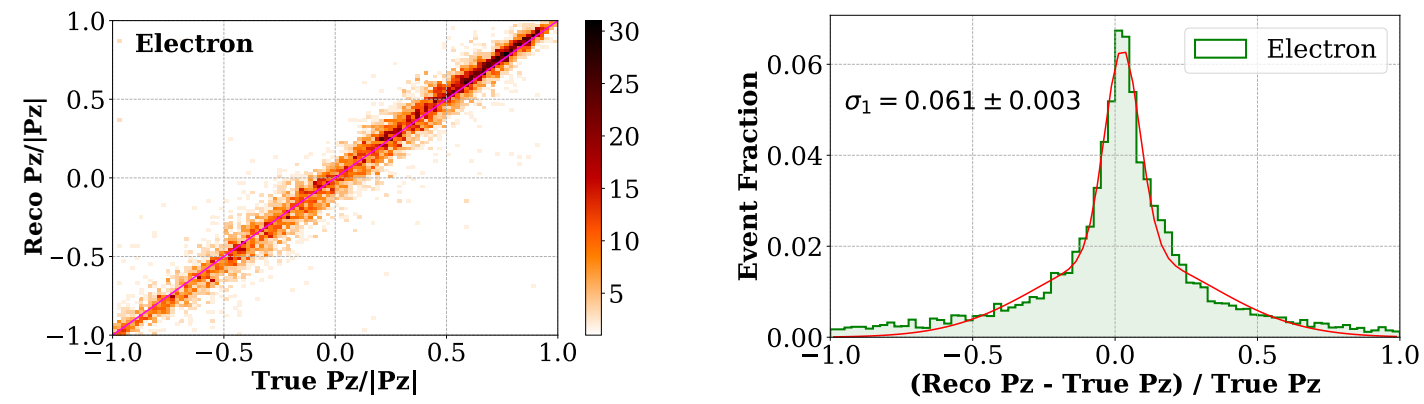

Figure 8.80: Right: Reconstructed versus true electron angle with respect to the beam for a sample of $1 e 1 \mathrm{P}$ events. The magenta line shows $y=x$ to characterize the bias. Left: Fractional difference between the reconstructed and true angle with a double gaussian fit. The angular resolution is approximately $6 \%$.

In low energy $1 e 1 \mathrm{P}$ events, the electron can take on any angle with respect to the beam axis. The fractional resolution of the electron and is approximately $6 \%$. There is no significant dependence of the electron shower direction with energy.

\subsubsection{Energy Reconstruction}

The electron and proton energies are estimated for $1 e 1 \mathrm{P}$ interactions by integrating all of pixels associated with the collection-plane belonging to the particle in the LArCV image. The summed pixel count, representing the underlying charge deposition, is converted to $\mathrm{MeV}$ by a linear calibration. The calibration for electron and proton particles for $1 e 1 \mathrm{P}$ interactions is shown in Fig. 8.81.

For each particle type, the integrated charge distribution is binned in both true deposited energy and summed pixel value. In each true energy bin a gaussian function is fit to the pixel value distribution. The mean position of the gaussian fit is shown as the blue dot with 

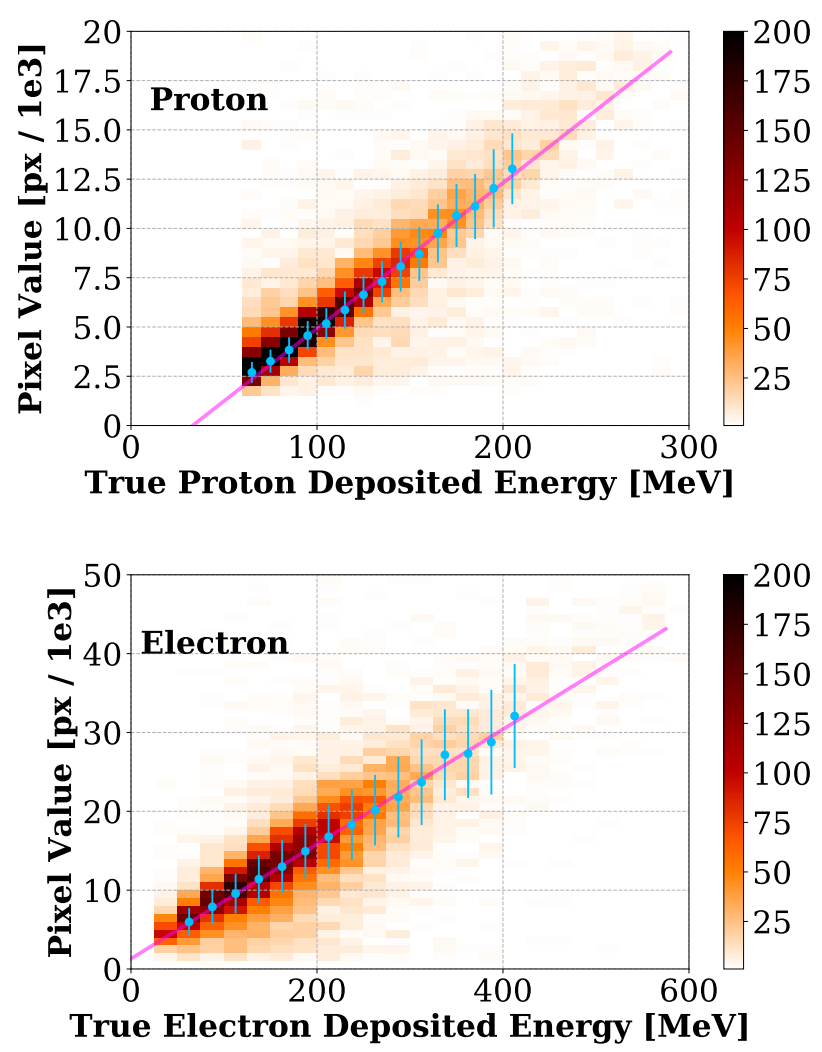

Figure 8.81: Proton (top) and electron (bottom) summed collection plane pixel value versus true deposited energy for low energy $1 e 1 \mathrm{P}$ events. In each true energy bin a gaussian is fit to the pixel value distribution to estimate the mean and spread (blue points). A linear fit (magenta line) is optimized for the blue points.

vertical uncertainty given by the standard deviation. A two parameter fit is optimized for the blue points. The slope from each particle fit are then averaged and a single parameter fit is optimized to determine the pixel value offset at $0 \mathrm{MeV}$.

The fit represents the calibration between the pixel and $\mathrm{MeV}$ scale. The relations for converting pixel to $\mathrm{MeV}$ scale for electron and proton particles are described as,

$$
\begin{aligned}
E_{\text {proton }}^{\text {cal }} & =\left(1 / 72.66 \times E_{\text {pixel }}+30.68\right) \mathrm{MeV} \\
E_{\text {electron }}^{\text {cal }} & =\left(1 / 72.66 \times E_{\text {pixel }}-27.14\right) \mathrm{MeV}
\end{aligned}
$$

A difference in the zero point energy can be attributed to a difference in charge recombina- 
tion between electron and proton particles. The fraction energy resolution for electron and proton particles is shown as a function of true particle energy in Fig. 8.82 .
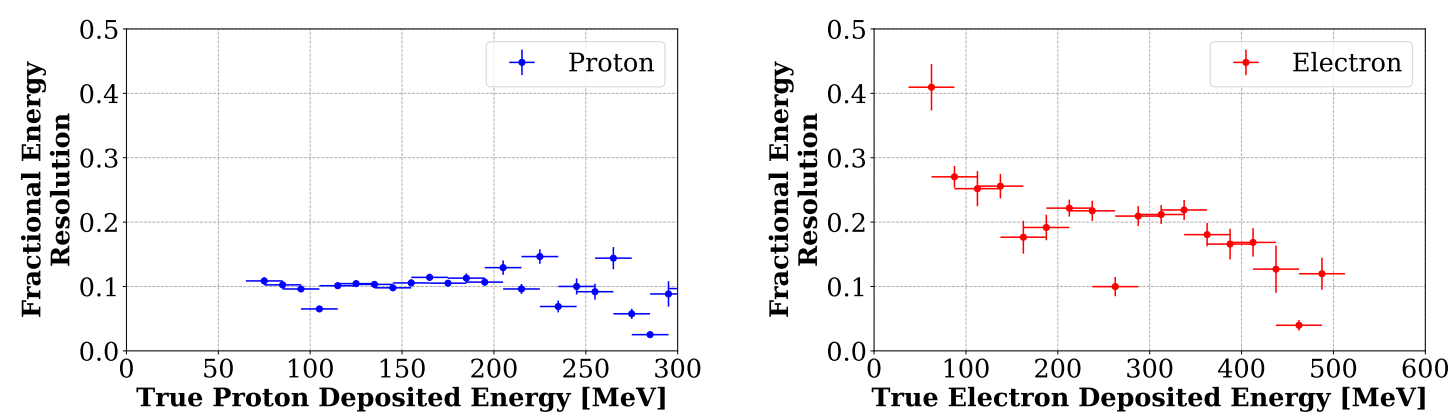

Figure 8.82: Fractional energy resolution for proton (left) and electron (right) particles as a function of true deposited energy. An approximately $10 \%$ and $20 \%$ energy resolutions are observed for proton and electron particles respectively using calorimetry.

Both proton and electron energy resolutions are approximately $10 \%$ and $20 \%$ respectively. In the method described above, an accurate estimate of the electron and proton energy can be determined without the need for hit based reconstruction.

\subsection{6 dE/dx Reconstruction}

The $d E / d x$ for $1 e 1 \mathrm{P}$ events is reconstructed by estimating the $d Q / d x$ as a function of length for each particle and applying a calibration factor. For each particle reconstructed at the vertex point, a 3D line is extended from the vertex point in the direction of the reconstructed particle direction. The line is segmented into units of $0.3 \mathrm{~cm}$ and projected onto each of the planes. Next, the charge is projected perpendicularly onto the line. In each segment the median pixel value is chosen and then corrected for the pitch of the track with respect to the wire plane. An example spectrum for a 1e1P interaction is shown in Fig. 8.83 ,

Proton and electron $d Q / d x$ spectra are reconstructed using a straight line hypothesis. The proton particle exhibits a larger baseline value than the electron, and a Bragg peak is typically observed. The example electron particle remains at a minimally ionizing value through a region of dead wires until its showering point. A truncated mean algorithm is applied to each particle's charge spectrum to smooth out local distortions in the charge 

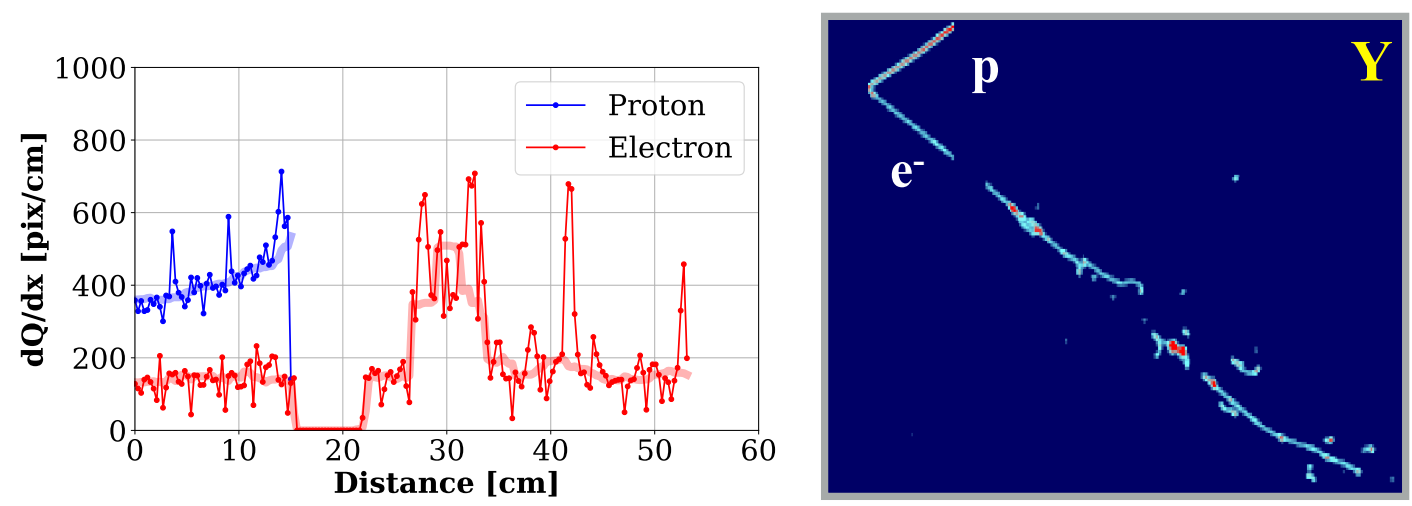

Figure 8.83: Left: $d Q / d x$ profiles as a function of particle length for proton (blue) and electron (red). The dotted line represents the binned charge deposition per length and the solid line is a truncated mean for smoothing. Right: The $1 e 1 \mathrm{P}$ events corresponding to the $d Q / d x$ profiles shown on the left.

profile. The $d Q / d x$ spectrum are converted to $d E / d x$ using the MeV scaling factor derived in Section 8.8.5 and by applying an average $d Q / d x$ spatial correction factor. The result is shown in Fig. 8.84 for a sample if low energy $1 e 1 \mathrm{P}$ events.

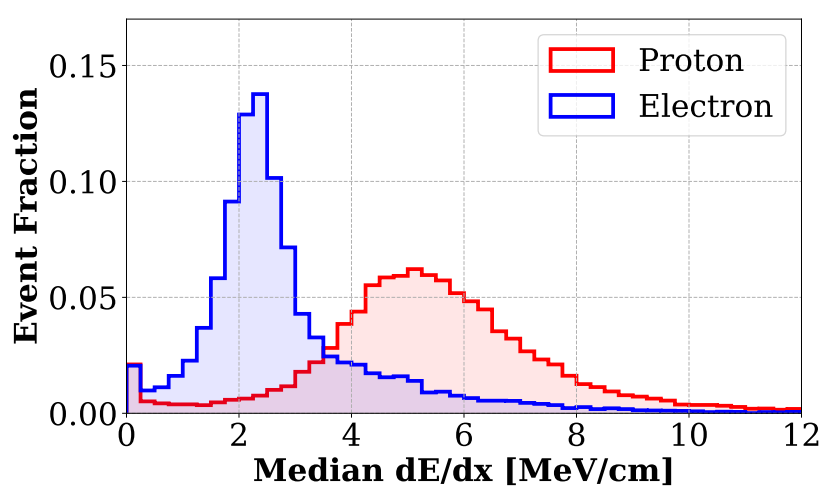

Figure 8.84: Electron (blue) and proton (red) median $d E / d x$ value for a simulated sample of $1 e 1 \mathrm{P}$ events. A median of $\sim 2.2 \mathrm{MeV} / \mathrm{cm}$ and $\sim 5 \mathrm{MeV} / \mathrm{cm}$ is observed.

The proton $d E / d x$ is estimated as the average $d E / d x$ over the full proton track while the electron $d E / d x$ is estimated using the first 4 centimeters to avoid including charge past an electrons showering point. The $d E / d x$ profile as a function of residual range for protons 
is shown in Fig. 8.85 .

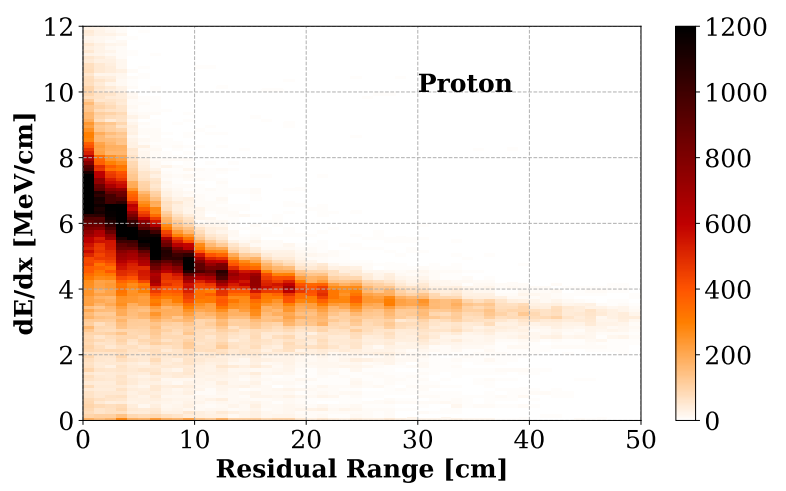

Figure 8.85: Proton $d E / d x$ as a function of residual range. An increase in charge deposition per unit length is observed toward the end of a proton's track.

An increase in charge deposition as the proton nears its end point is observed consistent with the Bethe-Bloch theory.

\subsubsection{Flash Matching}

The PMT optical system in MicroBooNE can be used for neutrino event reconstruction. By matching the observed photo-electron spectrum with objects reconstructed in the TPC, a good-ness of fit, $\chi_{\mathrm{PE}}^{2}$, can be calculated. The $\chi_{\mathrm{PE}}^{2}$ can be used in neutrino selection to determine if the interaction is consistent with the observed flash. For the 1L1P events, the lepton and proton $d E / d x$ profiles are reconstructed in 3D. The algorithm utilizes the full muon and proton reconstructed track as described in Section 8.8.6. In the case of the electron, a straight line hypothesis is used, as a detailed 3D reconstruction of the electron shower past the trunk point is unavailable. At each binned $d E / d x$ point, a library, called the Photon Library, is used to estimate the light yield per particle type and light hypothesis is estimated for each PMT in the optical system. The photon library is generated by segmenting the detector volume into "voxels" and simulating a photon density per voxel. The photons in each voxel are then tracked through Rayleigh scattering, reflection, and absorption to the PMTs. The result of the simulation is a library of voxel visibility information for each PMT. An cartoon flash match procedure for a $1 \mu 1 \mathrm{P}$ event is shown in Fig. 8.86 . 


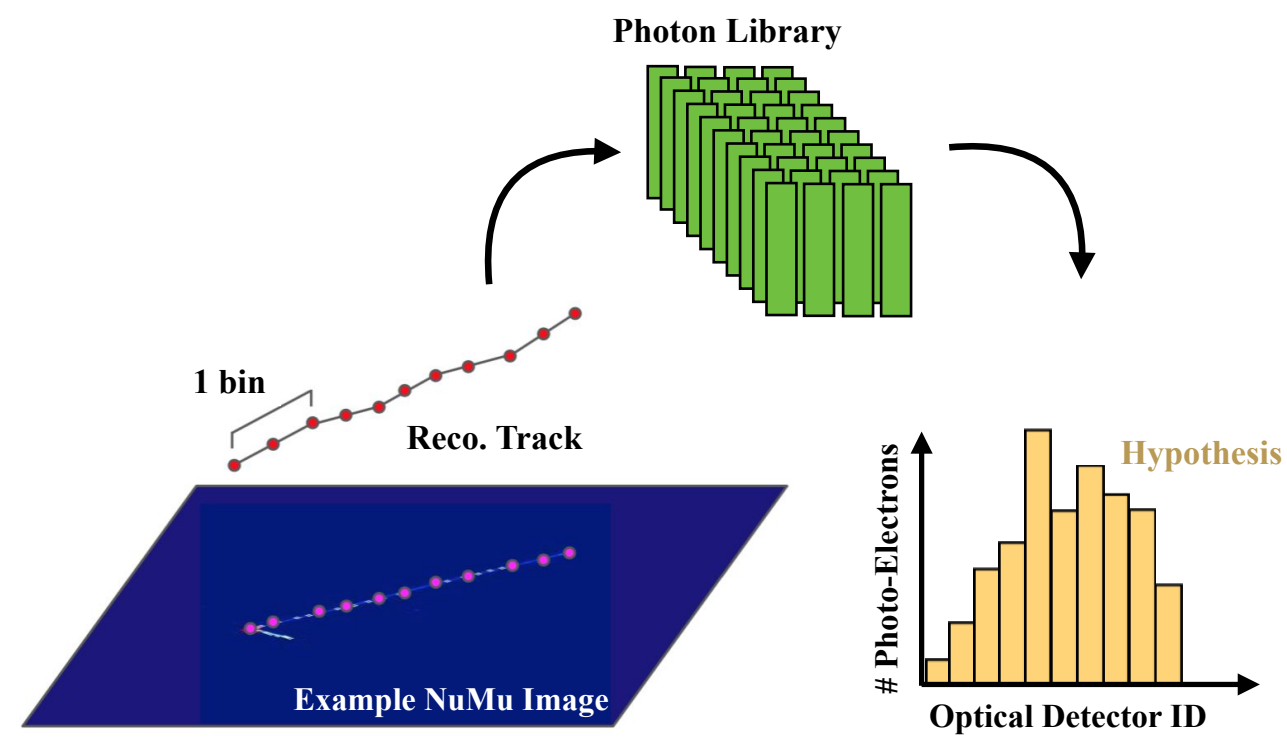

Figure 8.86: Cartoon of a flash hypothesis is constructed of a $1 \mu 1 \mathrm{P}$ interaction. A binned $d E / d x$ is reconstructed for the muon particle. Each charge deposition is converted to a light yield using the Photon Library forming a light hypothesis per PMT.

The hypothesis PE spectrum is then compared to the first reconstructed flash object for the beam window. A flash matching example for a well reconstructed $800 \mathrm{MeV} 1 e 1 \mathrm{P}$ interaction is shown in the left hand plot of Fig. 8.87 where a shape only $\chi_{\text {pe }}^{2}$ is formed between the hypothesis and the reconstructed (data) flash. A shape $\chi_{\mathrm{PE}}^{2}$ is better suited for the comparison since the absolute normalization of PE using the Photon Library contains uncertainty.

For well reconstructed $1 e 1 \mathrm{P}$ topologies we can expect $90 \%$ of the events to lie below a $\chi_{\mathrm{PE}}^{2}$ of approximately 2 as shown in the right hand plot in Fig. 8.87 .

\subsection{Particle ID using a Convolutional Neural Network}

\subsubsection{Network Architecture}

For single particle identification, two benchmark network architectures were studied: AlexNet and GoogLeNet. In addition, two down sampling schemes were profiled. The best result, defined as the highest particle identification accuracy over the five particles types, came 

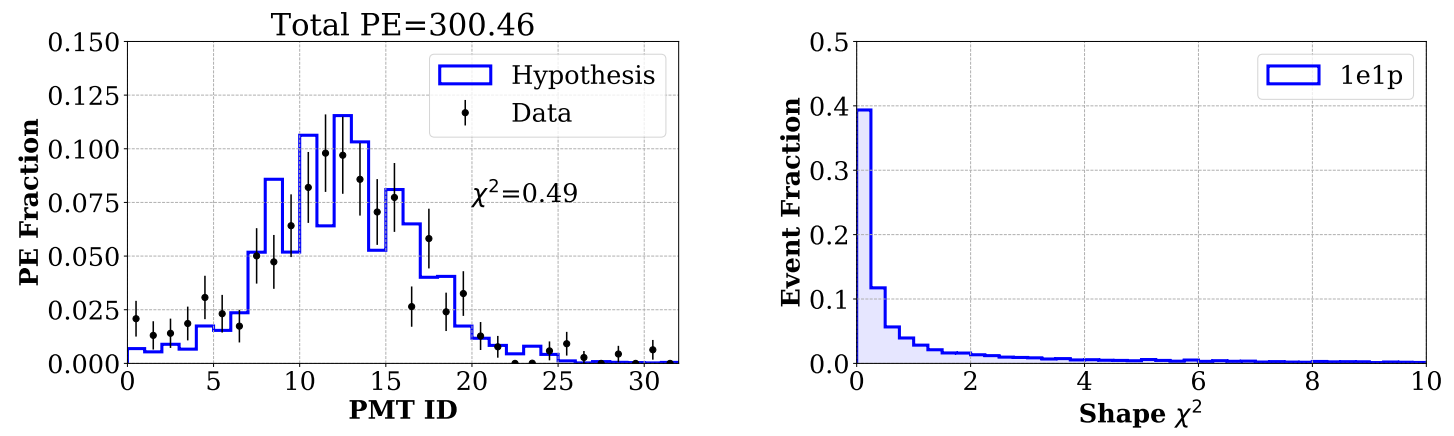

Figure 8.87: Example flash match for a 1e1P event. Left: The hypothesis (blue) spectrum is compared to the reconstructed flash (black) and a shape only $\chi^{2}$ is formed. Right: Distribution of shape only $c h i^{2}$ for a simulated sample of $1 e 1 \mathrm{P}$ events.

from using high resolution images with the GoogLeNet architecture. A new network, called VGG16b, has been optimized on high resolution LArTPC images for improved particle classification performance. An improvement of approximately 2 to 3 percentage points over AlexNet and GoogLeNet are shown in Table 8.3 .

\begin{tabular}{cccccc} 
& \multicolumn{5}{c}{ Classified Particle Type } \\
\cline { 2 - 6 } Network & $e^{-}[\%]$ & $\gamma^{-}[\%]$ & $\pi^{-}[\%]$ & $\mu[\%]$ & proton [\%] \\
\hline AlexNet & $73.6 \pm 0.8$ & $81.3 \pm 0.8$ & $84.8 \pm 0.5$ & $73.1 \pm 0.8$ & $87.2 \pm 0.5$ \\
GoogLeNet & $77.8 \pm 0.8$ & $83.4 \pm 0.7$ & $89.7 \pm 0.4$ & $71.0 \pm 0.8$ & $91.2 \pm 0.4$ \\
VGG16b & $82.9 \pm 0.7$ & $86.4 \pm 0.8$ & $90.7 \pm 0.4$ & $74.8 \pm 0.7$ & $90.8 \pm 0.4$ \\
\hline
\end{tabular}

Table 8.3: Single particle ID performance for high resolution LArTPC images for three CNN networks. AlexNet and GoogLeNet results reproduced from the reference while VGG16b results are new.

The Multi-PID network is implemented in the TensorFlow [42] framework and is shown in Fig. 8.88, The network architecture is modelled off of the VGG16b network and consists of 5 blocks of convolution with an increasing number of neurons (filters) separated by rectified linear units (ReLU) and average pooling procedures. Each convolution block retains a filter size of $3 \times 3$ and features a stride 2 convolution application in the first operation of each block. A single fully connected layer with 5 neurons is attached at the bottom of the network 
which outputs to a sigmoid function. The sigmoid function at the output layer models the probability of each particle being present in the image as a Bernoulli distribution, with each particle type being independent from one another. The Multi-PID network takes as input a $512 \times 512$ LArCV image, a crop centered at the reconstructed vertex, and returns a vector of length 5 with particle presence probabilities in the image.

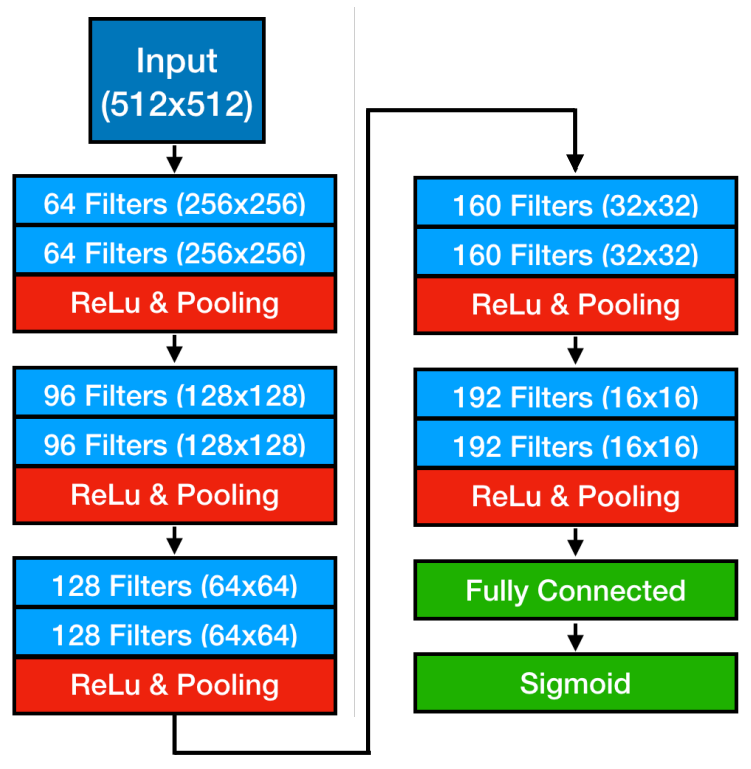

Figure 8.88: Multi-PID network architecture described in Section 8.9.1.

\subsubsection{Network Training}

The Multi-PID network is trained using a multi-particle generator simulation as described in Section 8.5.2. The generator produces simulated 3D interaction vertices at random locations inside the MicroBooNE TPC with no neutrino model dependence. Five types of particles are simulated: electron, gamma, muon, pion, and proton. The particles are emitted with isotropic momentum from the vertex with a random multiplicity of 1 to 4 . The data set consists of 95,000 images and contains the following energy distribution:

1. $80 \%$ of events are simulated with kinetic energy between 100 and $1000 \mathrm{MeV}$, except for proton which is simulated between 100 and $400 \mathrm{MeV}$.

2. $20 \%$ of events are simulated with kinetic energy between 30 and $100 \mathrm{MeV}$, except for 
proton which is simulated between 40 and $100 \mathrm{MeV}$.

The dataset is separated into a training set with 45,000 events and a testing set with 40,000 images. A batch size of 5 images is used for training. The network is trained using stochastic gradient descent for a period of 100 epochs for each 3 planes independently. The accuracy on the training and test samples, and the loss curve are shown in Fig. 8.89 .

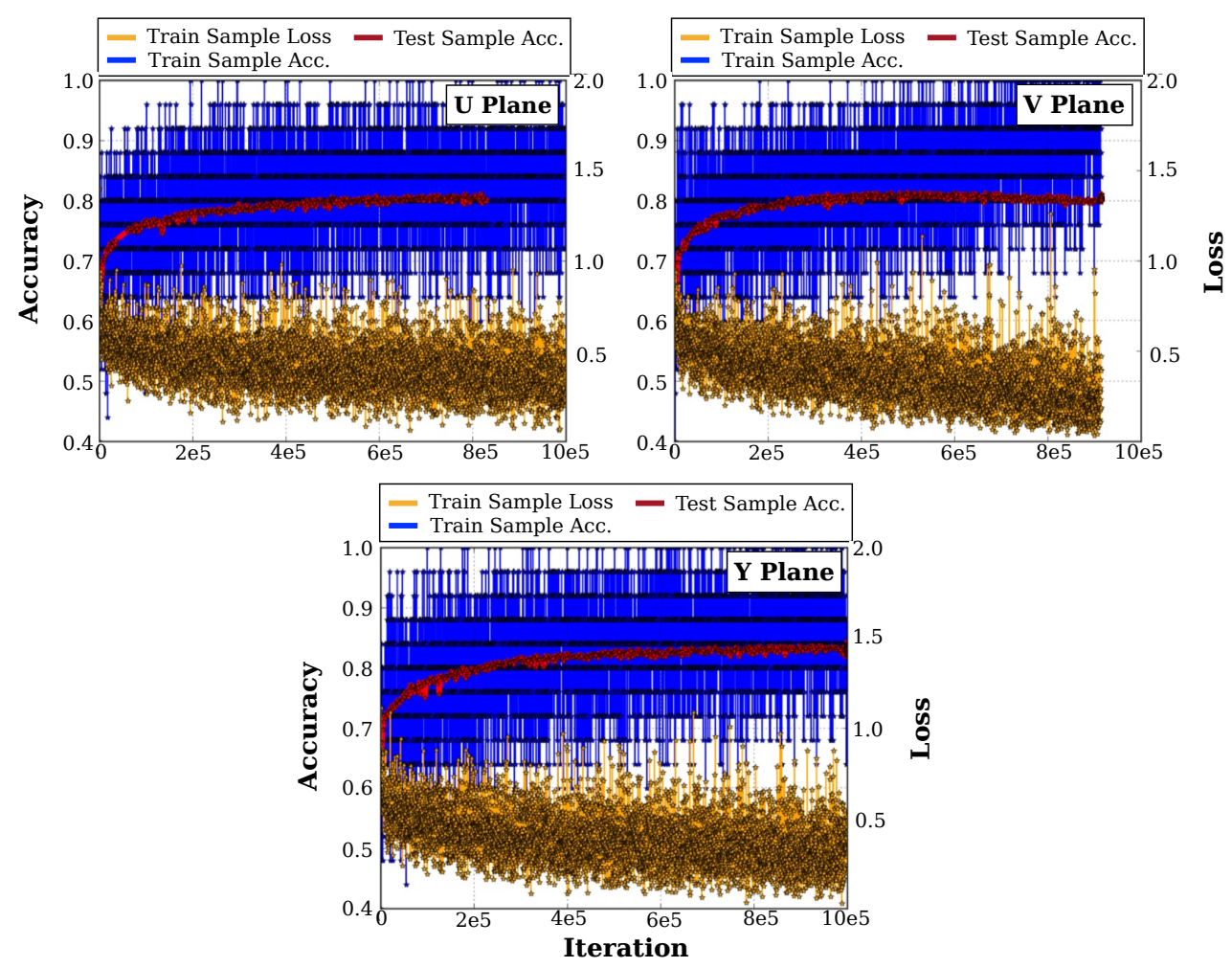

Figure 8.89: Network training for U (upper left), V (upper right), and Y (bottom) plane. The blue and red curves show the accuracy on the train and test samples respectively. The gold curve shows the loss value as a function of time.

The $\mathrm{U}$ and $\mathrm{V}$ induction planes exhibit plateaus in testing accuracy at approximately $80 \%$. Over training is observed on the $\mathrm{V}$ plane as training sample accuracy diverges from the testing sample accuracy toward the end of training. The Y plane performs the best with testing accuracy approaching 85\%. Weights for the Multi-PID network are selected 
before over training occurs at approximately 50 epochs.

\subsubsection{Performance}

The Multi-PID network is validated on simulated $1 e 1 \mathrm{P}$ and $1 \mu 1 \mathrm{P}$ events to determine the efficiency for identifying electron, muon, and proton particles. In practice, a cut on the network score value is used to determine the presence of particle type. The exact score value per particle type is optimized alongside hand designed features in Section 8.8 for neutrino selection. A reasonable score cut of 0.8 is used to claim the presence of a particle in an image. The network score distributions for 1L1P events on the $\mathrm{Y}$ plane are shown in Fig. 8.90.
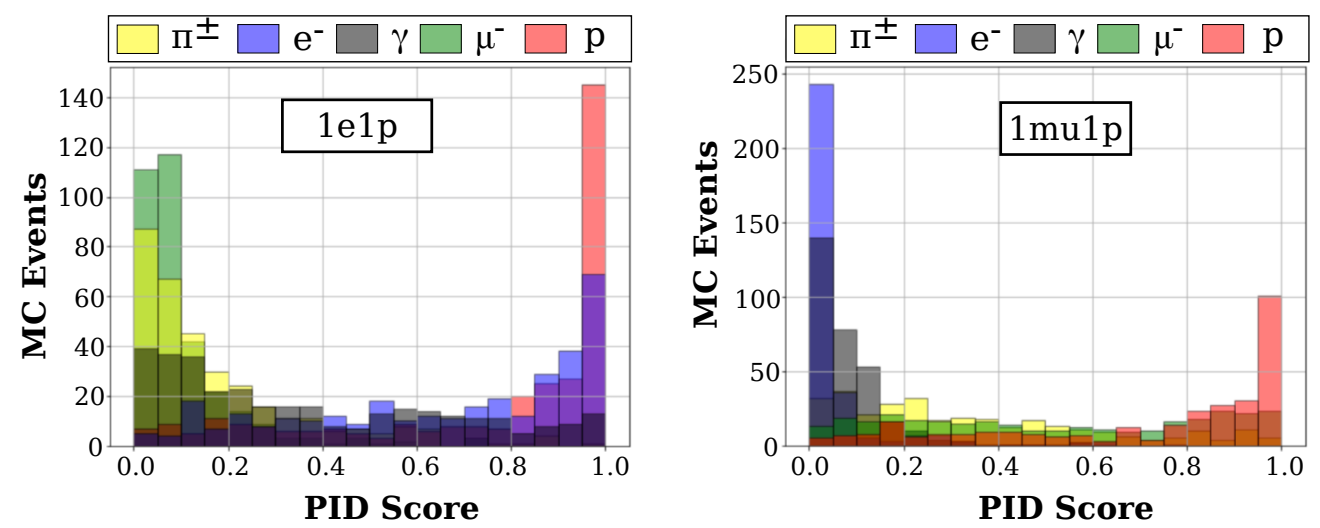

Figure 8.90: PID score distributions from the Multi-PID network for five particle types for $1 e 1 \mathrm{P}$ (left) and $1 \mu 1 \mathrm{P}$ (right).

For $1 e 1 \mathrm{P}$ approximately $62 \%$ of events have an electron score above 0.8 . The network exhibits the best response for identifying proton particles against the four others. Pion, muon, and gamma particles cluster below a score value of 0.2 . For $1 \mu 1 \mathrm{P}$ the network exhibits poor performance for identifying muon type particles as only $42 \%$ of events lie above a score value of 0.8. Studies have shown that muon particle score is strongly correlated with particle track length. As the muon decreases in length the network assigns lower scores and the overall distribution resembles that of pion scores. This provides evidence for the fact that muon and pion particles are challenging to distinguish using LArTPC images as they have similar features. As in the case of $1 e 1 \mathrm{P}$ events, the network exhibits the best response 
on proton particles.

Next the number of electron, muon, and proton particles in the interaction is varied from 0 to 2 to study how well the network can identify electron and muon particles given the event multiplicity. The mis-classification rate of the electron and muon with gamma and pion respectively are studied. The results for varying electron and proton multiplicity is shown in Table 8.4 .

\begin{tabular}{c|c|c|c} 
& $e>0.8$ & $\gamma>0.8$ & $e>\gamma$ \\
\hline $1 e 0 \mathrm{P}$ & 0.65 & 0.22 & 0.77 \\
$1 e 1 \mathrm{P}$ & 0.62 & 0.25 & 0.74 \\
$1 e 2 \mathrm{P}$ & 0.61 & 0.24 & 0.72 \\
$2 e 0 \mathrm{P}$ & 0.64 & 0.46 & 0.70 \\
$2 e 1 \mathrm{P}$ & 0.86 & 0.29 & 0.84 \\
$2 e 2 \mathrm{P}$ & 0.81 & 0.25 & 0.81
\end{tabular}

Table 8.4: Multi-PID network classification accuracy for low multiplicity electron neutrino interactions for a given topology. Values reported represent the fraction of events.

The network is more likely to identify an event as having an electron particle if more than one electron is present in the image as expected. If no protons are present at the vertex two electrons are most confused with the gamma type particle with $46 \%$ of events identifying the presence of a gamma above the score threshold. In general, the electron score is larger than the gamma score in approximately $75 \%$ of low multiplicity events.

The results for muon and proton events in Table 8.5 .

The muon has best classification when two muons alone are present in the image with no proton present. The muon exhibits some mis-classification with the pion particle when more than one muon is present in the image. In general, the muon score is larger than the pion score for each type of low multiplicity event topology. 


\begin{tabular}{c|c|c|c} 
& $\mu>0.8$ & $\pi>0.8$ & $\mu>\pi$ \\
\hline $1 \mu 0 \mathrm{P}$ & 0.59 & 0.06 & 0.79 \\
$1 \mu 1 \mathrm{P}$ & 0.42 & 0.17 & 0.65 \\
$1 \mu 2 \mathrm{P}$ & 0.43 & 0.25 & 0.54 \\
$2 \mu 0 \mathrm{P}$ & 0.86 & 0.42 & 0.79 \\
$2 \mu 1 \mathrm{P}$ & 0.76 & 0.42 & 0.65 \\
$2 \mu 2 \mathrm{P}$ & 0.69 & 0.46 & 0.46
\end{tabular}

Table 8.5: Multi-PID network classification accuracy for low multiplicity muon neutrino interactions for a given topology. Values are reported represent the fraction of events. 


\section{Chapter 9}

\section{Neutrino Event Selection}

This chapter will describe the $1 \mu 1 \mathrm{P}$ and $1 e 1 \mathrm{P}$ neutrino selections to reduce cosmic ray and neutrino induced backgrounds for the LEE search. At this stage multiple vertices have been found in the MicroBooNE event image. The distribution of reconstructed 1e1P events as a function of reconstructed neutrino energy (quantity described in chapter) after image based data reconstruction is shown in Fig. 9.1. Each candidate vertex represents a potential neutrino interaction or background which must be classified to obtain a pure sample of 1L1P events. Before selection is applied, cosmic ray backgrounds dominate the data sample. The $1 \mu 1 \mathrm{P}$ and $1 e 1 \mathrm{P}$ selection procedures follow a separate multivariate approach to select the most likely candidate vertex per event which represents the desired neutrino flavor. The $1 \mu 1 \mathrm{P}$ selection applies a two log-likelihood scheme, a cosmic and neutrino discriminator, to identify the best candidate vertex per event. The $1 e 1 \mathrm{P}$ selection pursues a machine learning based classification scheme using a combination of Boosted Decision Tree (BDT) and Support Vector Machine (SVM) algorithms. 1L1P reconstructed energy and kinematic distributions are compared to the $5 \times 10^{19}$ POT MicroBooNE Run 1 data set. The $1 e 1 \mathrm{P}$ rate prediction is shown for $13.2 \times 10^{20} \mathrm{POT}$. 

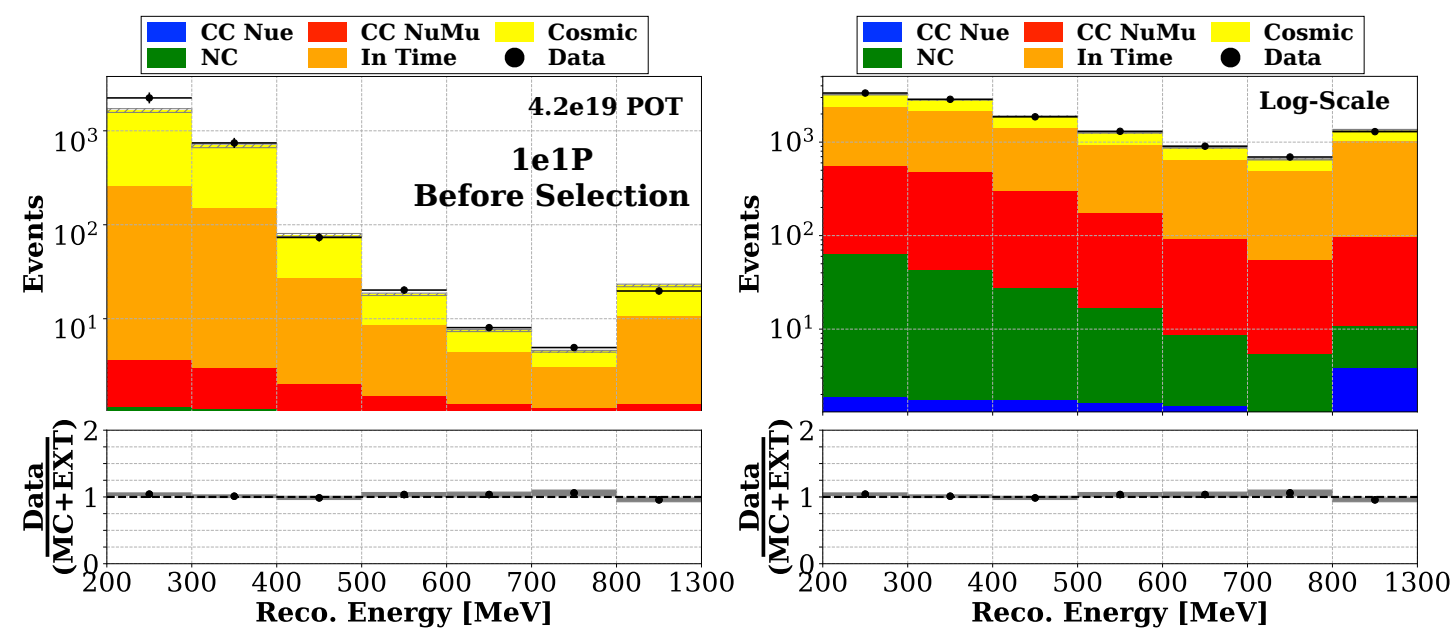

Figure 9.1: The 1e1P stacked background prediction as a function of reconstructed neutrino energy for estimating the $5 \times 10^{19}$ POT open data set before selection as described in Chapter 9. Black dots represents Run 1 detector data. The gray boxes represent MC statistical uncertainty. The cosmic ray contamination (yellow and orange distributions) for the $1 e 1 \mathrm{P}$ search is high. Selection is applied in this chapter to reduce cosmic and neutrino induced backgrounds. Other distributions are described in the text. Data are shown in linear scale (left) and logarithmic scale (right).

\section{$9.1 \quad 1 \mu 1 \mathrm{P}$ Selection}

\subsubsection{Overview}

An event selection based on the $3 \mathrm{D}$ tracking algorithm is developed to isolate $1 \mu 1 \mathrm{P}$ events against background. The primary background are two track topologies reconstructed from candidate vertices found on cosmic rays which feature two tracks emitted from a common $3 \mathrm{D}$ origin. A secondary background are from neutrino interactions which do not satisfy the $1 \mu 1 \mathrm{P}$ criteria (e.g. multiple protons, or tracks which exit the active volume). The event selection involves two components. The first component is a series of sequential rectangular cuts on 3D vertex and track features. The second component is a log-likelihood based discriminant which includes eight features to separate the $1 \mu 1 \mathrm{P}$ topology against cosmic ray and neutrino backgrounds.

The selection is benchmarked on a Monte Carlo generated sample of BNB interactions 
equivalent to $6.6 \times 10^{20} \mathrm{POT}$, referred to as the cocktail sample. The cocktail sample contains events with a GENIE simulated neutrino interaction (version 2.12.2, MCC8 era) overlaid with externally unbiased data cosmic ray events. The cosmic ray data overlay provides real cosmic ray data from beam off detector data taking and includes many detector effects not modelled in simulation such as some classes of wire noise. The overlay sample features real TPC signal formation which effects the topological shape of charge depositions in the image. Additionally, the cosmic ray background rate and distribution on the detector edges is better estimated. The event rate prediction is compared to the $5 \times 10^{19} \mathrm{POT}$ data set in reconstructed neutrino energy and kinematic variables to asses the Data-MC agreement.

The dominant background to the $1 \mu 1 \mathrm{P}$ selection are cosmic rays reconstructed in events which do not contain a neutrino interaction. The second most prevalent background involves events where one of the outgoing particles fails to be reconstructed such as topologies with additional protons or charged pions.

\subsubsection{Pre-cuts}

The event selection performs a series of rectangular cuts on vertex and track reconstructed features to reduce the cosmic ray background before likelihood selection and are described below.

\section{Fiducial Volume}

The reconstructed vertex location, $(x, y, z)$, is required to be located inside a $10 \mathrm{~cm}$ fiducial volume (FV) defined as $x \in[10,246.25] \mathrm{cm}, y \in[-106.5,106.5] \mathrm{cm}$, and $z \in[10,1026.8]$ $\mathrm{cm}$. The FV cut removes candidate vertices reconstructed close to the edge of the detector where one particle is likely to exit and feature information about the track may be incomplete.

\section{Two Track Requirements}

Exactly two tracks of length larger than 5 centimeter must be reconstructed at the candidate vertex point. The $1 \mu 1 \mathrm{P}$ signal definition is a two pronged topology with energy threshold set such that both the proton and muon are larger than $5 \mathrm{~cm}$ in length. This 
analysis cut explicitly removes charged current interactions where additional protons or pions exit the vertex.

Both tracks must be fully contained inside the active volume. Candidate vertices are vetoed if at least one of the tracks end point approaches within 15 centimeters of the detector boundary.

The muon and proton particle are identified using the average pixel intensity value of each of the tracks as shown in distribution shown in Fig. 8.65. The track with the largest average pixel intensity is labelled as the proton particle, and the smallest as the muon.

\section{Positive Track Diagnostic}

The tracking algorithm features an internal self diagnostic algorithm to categorize the quality of the reconstructed track. This diagnostic is called the track "goodness". The goodness of a track takes into account the ability for the track to reach a suitable end point by evaluating the quality of the TPC signal used to generate the track, whether the waveform is faint or interrupted, or the charge depositions being tracked are relatively straight. Two good tracks are required to be reconstructed at the candidate vertex.

\section{Quasi-Elastic Energy Consistency}

The 1L1P topology consists primarily of CCQE events which have a kinematic correlation between the emitted proton and lepton. Three estimates of the neutrino interaction energy for CCQE events are,

$$
\begin{aligned}
E_{\nu}^{\mathrm{range}} & =\mathrm{E}_{p}+E_{\mu}+M_{\mu}+M_{p}-\left(M_{n}-B\right) \\
E_{\nu}^{Q E}[p] & =\frac{1}{2} \frac{2\left(M_{n}-B\right) \cdot E_{p}-\left(\left(M_{n}-B\right)^{2}+M_{p}^{2}-M_{\mu}\right)}{\left(M_{n}-B\right)-E_{p}+\sqrt{\left(E_{p}^{2}-M_{p}^{2}\right) \cdot \cos \theta_{p}}} \\
E_{\nu}^{Q E}[\mu] & =\frac{1}{2} \frac{2\left(M_{n}-B\right) \cdot E_{\mu}-\left(\left(M_{n}-B\right)^{2}+M_{\mu}^{2}-M_{p}\right)}{\left(M_{n}-B\right)-E_{\mu}+\sqrt{\left(E_{\mu}^{2}-M_{\mu}^{2}\right) \cdot \cos \theta_{\mu}}}
\end{aligned}
$$

where $E_{\nu}^{\text {range }}, E_{\nu}^{Q E}[p], E_{\nu}^{Q E}[\mu]$ are the range based energy, and the quasi-elastic energy estimated from the proton and muon particles respectively. For each energy variable, the kinetic energy of the proton $E_{p}$ and the muon $E_{\mu}$ are estimated from the reconstructed track length as described in Section 8.7.4. A $40 \mathrm{MeV}$ effective nuclear binding energy, $B$, as 
well as the masses of the proton, $M_{p}$, muon, $M_{\mu}$, and the neutron $M_{n}$. The reconstructed angle of the proton and muon with respect to the beam, $\cos \theta_{p}$ and $\cos \theta_{\mu}$, are used.

The consistency between each estimated neutrino energy is used to isolate CCQE events against cosmic and non-CCQE neutrino backgrounds. Three analysis variables are defined by taking the difference between pairs of estimated reconstructed neutrino energy,

$$
\begin{aligned}
\Delta_{p \mu} & =E_{\nu}^{Q E}[p]-E_{\nu}^{Q E}[\mu], \\
\Delta_{\text {range }-\mu} & =E_{\nu}^{Q E}[p]-E_{\nu}^{\text {range }}, \\
\Delta_{\text {range }-p} & =E_{\nu}^{Q E}[p]-E_{\nu}^{\text {range }} .
\end{aligned}
$$

A requirement on the sum squared of $\Delta \mathrm{s}$ is,

$$
\Delta_{p \mu}^{2}+\Delta_{\text {range }-\mu}^{2}+\Delta_{\text {range }-p}^{2}<1 \mathrm{GeV}^{2}
$$

\section{Transverse Momentum Variables}

To exploit the nature of quasi-elastic scattering kinematics, the transverse kinematic imbalance can be used as a discriminant by considering momentum conservation in the transverse plane. Three single-transverse variables (STV) [5] are defined as,

$$
\begin{aligned}
\delta p_{\mathrm{T}} & =\vec{p}_{\mathrm{T}}^{\vec{\mu}}+p_{\mathrm{T}}^{\vec{p}}, \\
\delta \phi_{\mathrm{T}} & =\cos ^{-1}\left(\frac{\overrightarrow{p_{\mathrm{T}}^{\mu}} \cdot \overrightarrow{p_{\mathrm{T}}^{p}}}{p_{\mathrm{T}}^{\mu} p_{\mathrm{T}}^{p}}\right), \\
\delta \alpha_{\mathrm{T}} & =\cos ^{-1}\left(\frac{\overrightarrow{p_{\mathrm{T}}^{\mu}} \cdot \delta \vec{p}_{\mathrm{T}}^{p}}{p_{\mathrm{T}}^{\mu} \delta p_{\mathrm{T}}}\right),
\end{aligned}
$$

where $\delta p_{\mathrm{T}}$ is the overall 3 -momentum imbalance in the transverse plane and is generated by nuclear effects, $\delta \phi_{\mathrm{T}}$ is the angular difference between the final state particles if the particles were back to back in the transverse plane, and $\delta \alpha_{\mathrm{T}}$ is defined by the direction of the transverse momentum imbalance in relation to the axis defined by the muon. A diagram of the STV are shown in Fig. 9.2. The distributions for both $\delta p_{\mathrm{T}}$ and $\delta \phi_{\mathrm{T}}$ are shown in Fig. 9.3 . 


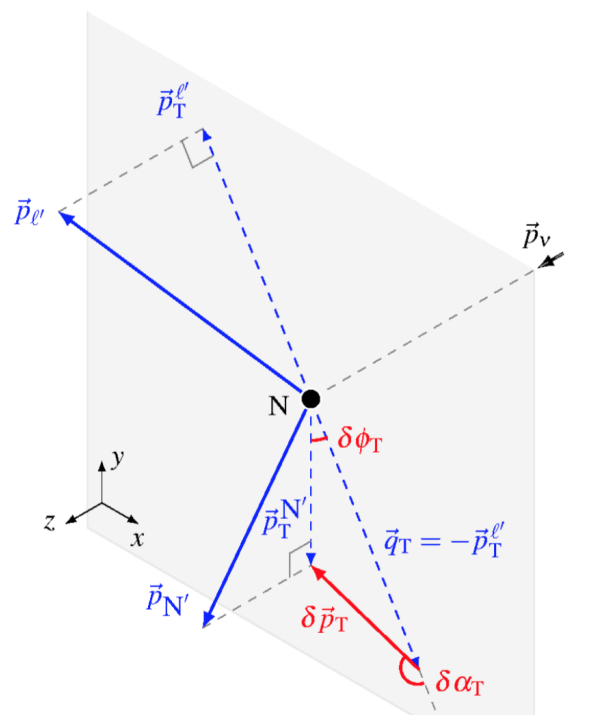

Figure 9.2: Schematic of single-transverse kinematic imbalance [5].
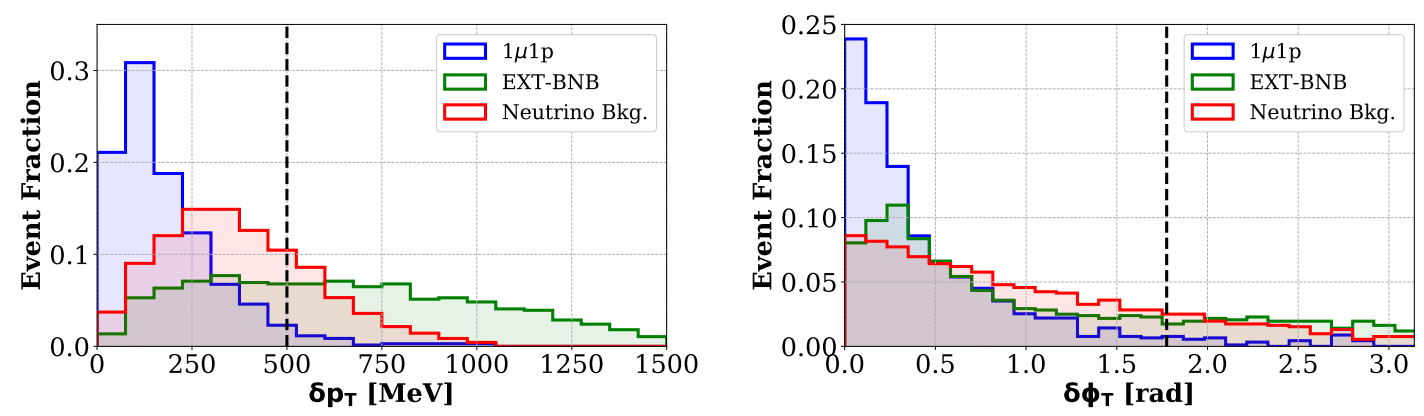

Figure 9.3: Transverse momentum variables $\delta p_{\mathrm{T}}$ and $\delta \phi_{\mathrm{T}}$ described in Section 9.1.2. Three distributions are shown for $1 \mu 1 \mathrm{P}$ events (blue), EXT-BNB events (green), and neutrino backgrounds events (red). Black dashed line indicates the location of the cut value.

The $1 \mu 1 \mathrm{P}$ events cluster around zero transverse momentum with small transverse angular deflection with some smearing due to nuclear effects present. A cut on $\delta p_{\mathrm{T}}<500 \mathrm{MeV}$ and $\delta \phi_{\mathrm{T}}<3 \pi / 8$ is applied.

\section{Four Momentum Transfer}


The magnitude of the four momentum transfer for the interaction is computed via,

$$
Q^{2}=2 E_{\nu}^{Q E}\left(E_{\mu}-p_{\mu} \cos \theta_{\mu}\right)-m_{\mu}^{2}
$$

and is expected to be larger than 0 for pure CCQE scattering. The distribution of $Q^{2}$ is shown in Fig. 9.4 for a sample of $1 \mu 1 \mathrm{P}$ events and the two primary backgrounds, EXT-BNB and neutrino backgrounds. Due to final state interactions, and the presence of MEC events

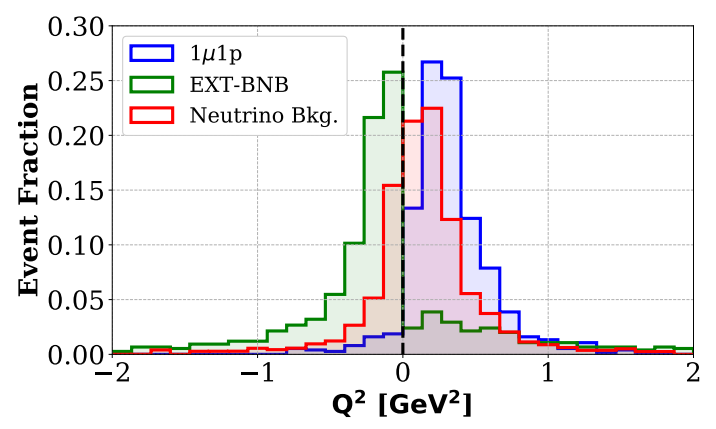

Figure 9.4: Four momentum transfer, $Q^{2}$, distribution for $1 \mu 1 \mathrm{P}$ events (blue), EXT-BNB events (green), and neutrino background events (red). Black dashed line indicates the location of the cut value.

in the $1 \mu 1 \mathrm{P}$ topology, a small population of events is found below 0 . Cosmic ray events feature $Q^{2}$ values below 0 as the two out going tracks do not represent neutrino interactions.

\section{Shower Activity}

Finally, two discriminants are formed by using the SSNet to detect the presence of shower activity at the candidate vertex location. Two track clusters are expected in a $1 \mu 1 \mathrm{P}$ interaction. The candidate vertex is rejected if a track follows a clustered particle with greater than $50 \%$ of its pixels tagged as shower like by the SSNet. This requirement reduces the intrinsic $\nu_{e}$ background before applying the likelihood selection in the following section. The second discriminant requires that there be no detached showers in a fixed region around the candidate vertex. The observation of detached showers indicates the potential presence of $\pi^{0}$ activity and are rejected. At the present moment, the reconstruction chain is unable to quantify energy associated with detached particles emitted from the vertex location. 


\subsubsection{Likelihood Selection}

A binned log-likelihood (LL) function is trained to select the candidate vertex per event which best represents the $1 \mu 1 \mathrm{P}$ interaction. A LL function is defined as,

$$
L L(E)=\sum_{i}^{N} \log \left(\frac{s_{i}}{b_{i}}\right),
$$

where $E$ is a vector of $N$ parameters, and $s_{i}$ and $b_{i}$ are the corresponding probabilities for the parameter $E_{i}$ to signal or background respectively. The distributions of $s_{i}$ and $b_{i}$ are discrete probability density functions (PDFs) which are constructed from signal and background populations. For a given vertex each parameter, $E_{i}$, of $E$ is reconstructed. Each parameter is look-ed up in the corresponding PDF and the ratio of the probability for the parameter to be signal to background is computed. The ratio is analogous computing the bayes factor for two competing hypothesis. The log probability is summed over $N$ parameters and the output, $L L(E)$, quantifies the likelihood for the vertex to be signal as opposed to background. The LL function returns a single score per reconstructed vertex which will determine the likelihood of being a $1 \mu 1 \mathrm{P}$ interaction versus a cosmic or neutrino background. A cut on the LL output is used to further reduce cosmic and neutrino backgrounds. The score is maximized to select the most likely $1 \mu 1 \mathrm{P}$ interaction.

Two likelihood functions are trained to discriminate $1 \mu 1 \mathrm{P}$ versus a cosmic ray, called the Cosmic LL, and other neutrino backgrounds, called the Neutrino LL. The LL functions are designed by considering three sample of events. First is a signal sample which consists of well reconstructed $1 \mu 1 \mathrm{P}$ events. Second is a cosmic background sample, represented by EXT-BNB events which pass the pre-cuts defined in the previous section. Third is a neutrino background sample, consisting of candidate vertices which pass the pre-cuts defined in the previous section and which have reconstructed vertices within 5 centimeters of the true vertex. Eight parameters are used to train the LL functions and are described below.

\section{dQ/dx Asymmetry:}

A $1 \mu 1$ p interaction vertex has one highly ionizing proton attached to a minimum ionizing muon. This feature is quantified by taking the ratio of the average charge deposition per unit length for the candidate muon and proton particle, referred to as $\eta$. For each reconstructed 
track, an average $d Q / d x$ is computed over the full track length. The charge collection efficiency varies spatially as described in Section 8.7.5 so the absolute magnitude of a proton or muon's $d Q / d x$ may not be consistent at all points in the detector. To account for spatial variation the asymmetry between the muon and proton $d Q / d x$ is calculated as,

$$
\eta=\frac{\left(\frac{d Q}{d x}\right)_{p}-\left(\frac{d Q}{d x}\right)_{\mu}}{\left(\frac{d Q}{d x}\right)_{p}+\left(\frac{d Q}{d x}\right)_{\mu}}
$$

The distribution of $\eta$ is shown in Fig. 9.5.

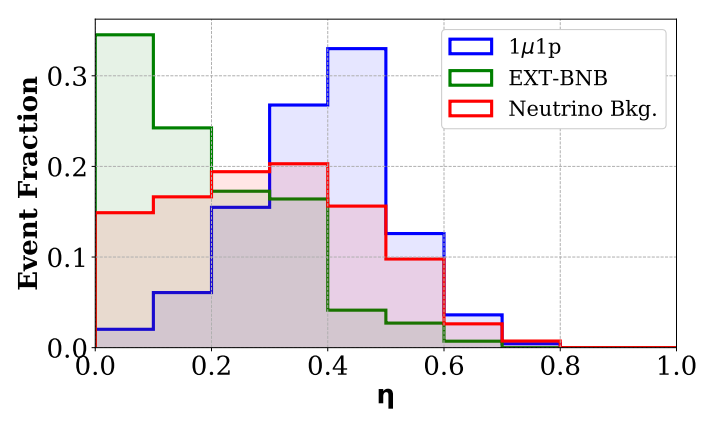

Figure 9.5: Charge deposition per unit length asymmetry, $\eta$, between the proton and muon track. Three distributions are shown: $1 \mu 1 \mathrm{P}$ (blue), EXT-BNB (green), and neutrino background (red).

As expected, the cosmic ray background events concentrate at lower values of $\eta$ as the associated particles are two muons. Some discrimination is observed against neutrino backgrounds which may contain a charged pion.

\section{Track Kinematics:}

Track kinematic parameters are used to aid in identifying CCQE-like scattering. The three single transverse variables, $\delta p_{\mathrm{T}}, \delta \phi_{\mathrm{T}}$, and $\delta \alpha_{\mathrm{T}}$ described in the previous section are used as input into the likelihood. Additionally three kinematic parameters, as shown in Fig. 9.6, characterize scattering in the longitudinal plane are quantified.

First is the 3D opening angle between the proton and muon particle. For CCQE scattering a vee shape topology will be present. For cosmic ray backgrounds particles will be 

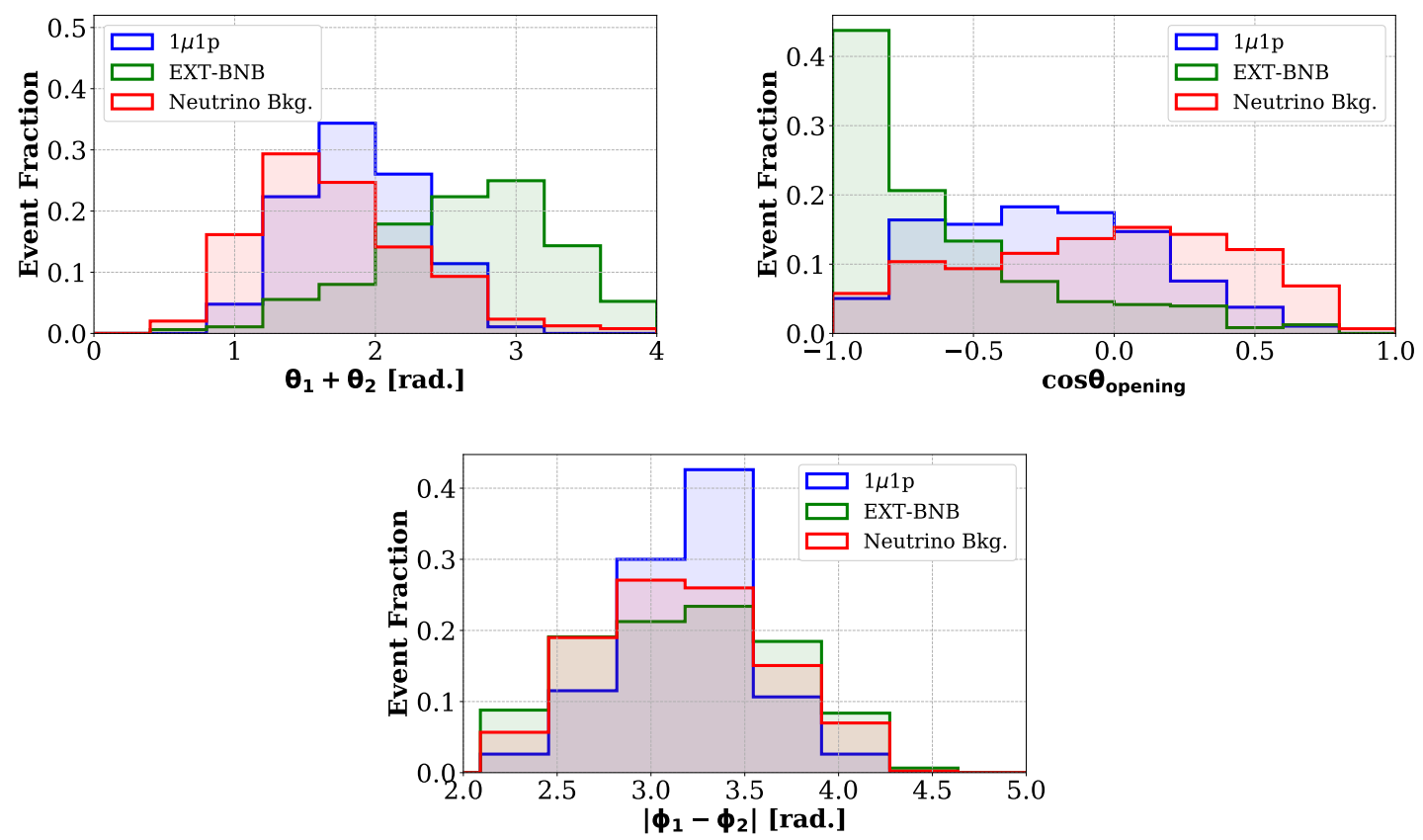

Figure 9.6: Track kinematic variables described in Section 9.1.3. Three distributions are shown: $1 \mu 1 \mathrm{P}$ (blue), EXT-BNB (green), and neutrino background (red)

emitted back to back or feature large, obtuse opening angles. Neutrino backgrounds with multi-body final states are distinguished from $1 \mu 1 \mathrm{P}$ events by featuring a larger fraction of acute opening angles. The angular expectations for $1 \mu 1 \mathrm{P}$ events are captured by computing the sum of the track thetas, or angle with respect to the beam line, which should be forward biased for beam induced events, and by computing the absolute difference in $\phi$ angle, which should peak at $\pi$ radians for two body events.

\section{Bjorken $\mathrm{x}$ :}

The final input into the log likelihood function is the Bjorken scaling factor computed as,

$$
x=\frac{Q^{2}}{2 M_{p} M_{\nu}},
$$

where $Q^{2}$ is the momentum transfer, $M_{p}$ is the proton mass, and $M_{\nu}=E_{\nu}^{\mathrm{range}}-E_{\mu}$. The Bjorken scaling factor corresponds to the fraction of proton momentum carried by the parton which takes part in an elastic scattering process. The scaling factor for the three 
samples is shown in Fig. 9.7. For CCQE scattering the Bjorken scaling factor has a value of

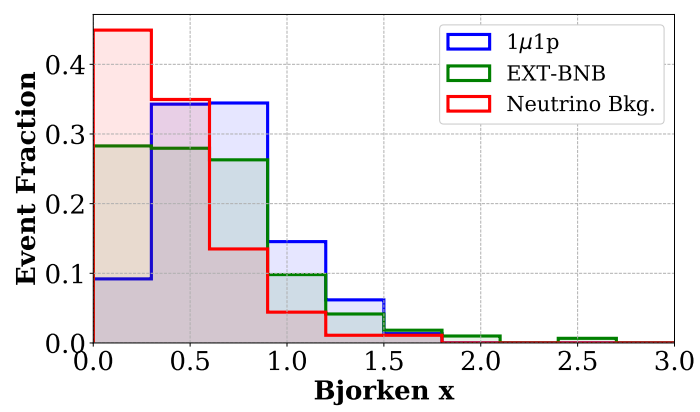

Figure 9.7: Bjorken scaling factor $\mathrm{x}$ for three samples: $1 \mu 1 \mathrm{P}$ (blue), EXT-BNB (green), and neutrino background (red).

one (completely elastic) as the squared momentum transfer matches the twice product of the proton mass and energy difference between the neutrino and the kinetic energy of the muon. A bias is observed due to reconstruction effects.

The two log likelihood functions are trained and independently evaluated on a sample of signal and background events and are shown in Fig. 9.8. The Cosmic LL distribution
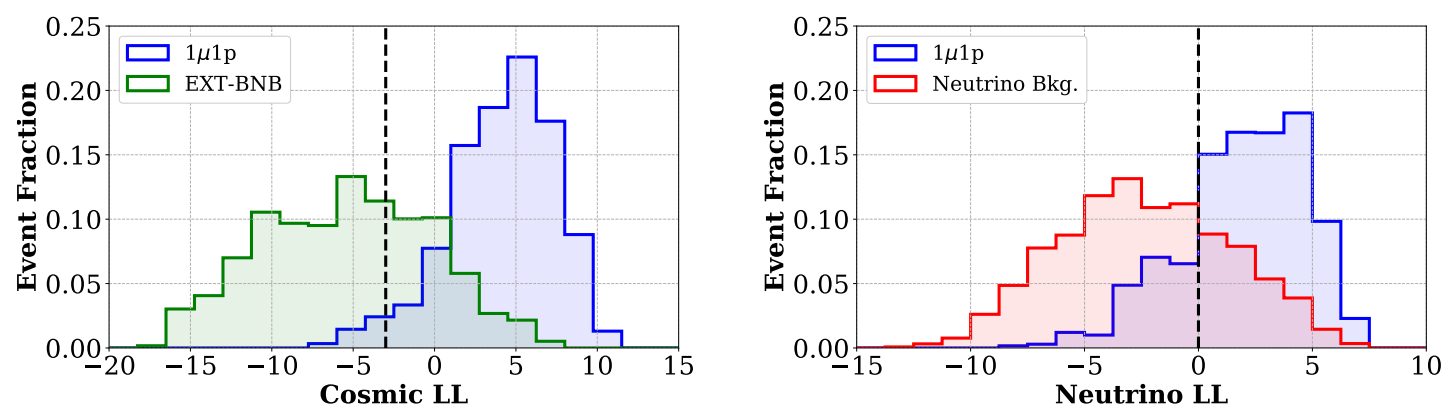

Figure 9.8: Log-likelihood distributions for discriminating $1 \mu 1 \mathrm{P}$ events against cosmic rays (left) and neutrino backgrounds (right). The black dashed line represent the LL cut value to categorize a signal versus background.

shows good separation between signal and background. A rectangular cut value of -3 is placed on the Cosmic LL to distinguish signal. The distribution of Neutrino LL exhibits 
weaker discrimination where a score cut of 0 is applied. The candidate neutrino vertex with the highest Cosmic LL score above threshold is considered the $1 \mu 1 \mathrm{P}$ interaction.

The efficiency of the Pre-cuts and the LL procedure is shown as a function of true neutrino energy in Fig. 9.9. The resulting selection is approximately $18 \%$ efficient and $63 \%$ pure of $1 \mu 1 \mathrm{P}$ events after both reconstruction and final selection is applied.

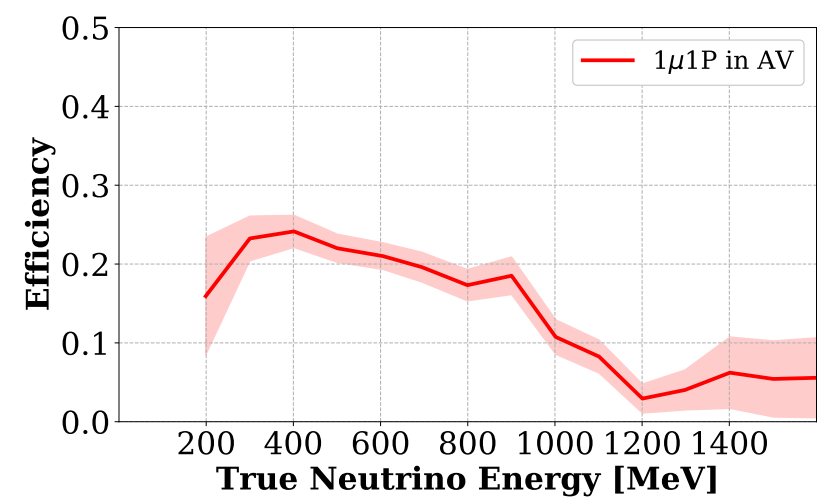

Figure 9.9: $1 \mu 1 \mathrm{P}$ selection efficiency as a function of true neutrino energy for interactions located in the active volume. A downward trend in efficiency is observed above $800 \mathrm{MeV}$ as muons reach large enough energy to exit the detector. A maximum efficiency of approximately $20 \%$ is achieved in the sub $600 \mathrm{MeV}$ region.

The efficiency of the rectangular cuts in the precut stage is shown in Table 9.1. The global efficiency for finding a $1 \mu 1 \mathrm{P}$ vertex is approximately $60 \%$ and is described in Section 8.6.6. After track reconstruction, and applying the rectangular and LL cuts the efficiency drops to $18 \%$. The background rejection capability for EXT-BNB and neutrino background events is $99.97 \%$ and $98.7 \%$ respectively. The dominant backgrounds are neutrino induced topologies which feature a charged pion or additional protons above $60 \mathrm{MeV}$ which fail to be reconstructed. A breakdown of the various backgrounds are described in the following section.

\subsubsection{Data-MC Comparison}

The $1 \mu 1 \mathrm{P}$ selection is applied to the MicroBooNE Run 1 dataset, equivalent to $4 \times 10^{19}$ POT after data quality cuts have been applied. Two samples are used to estimate the 


\begin{tabular}{|c|c|c|c|}
\cline { 2 - 4 } \multicolumn{1}{c|}{} & \multicolumn{3}{c|}{ Efficiency [\%] } \\
\cline { 2 - 4 } \multicolumn{1}{c|}{} & $\mathbf{1} \mu \mathbf{1 P}$ & EXT-BNB & Neutrino Bkg. \\
\hline $\begin{array}{c}\text { Fiducial Volume, } \\
\text { Two Track, } \\
\text { \& Positive Track Diagnostic }\end{array}$ & 26 & 2.9 & 5.0 \\
\hline $\begin{array}{c}\text { Quasi-Elastic Energy } \\
\text { Consistency }\end{array}$ & 22 & 0.6 & 3.1 \\
\hline $\begin{array}{c}\text { Transverse Momentum Variables, } \\
\text { \& Four Momentum Transfer }\end{array}$ & 21 & 0.3 & 2.1 \\
\hline Shower Activity & 20 & 0.2 & 1.8 \\
\hline Likelihood Selection & 18 & 0.03 & 1.3 \\
\hline
\end{tabular}

Table 9.1: $1 \mu 1 \mathrm{P}$ selection efficiency for stages of cuts described in Section 9.1.3

rate of neutrino and cosmic ray backgrounds. The cocktail neutrino sample quantifies the neutrino content of the selected sample and represents the events where neutrinos interact in the beam spill window. A sample of cosmic rays from externally triggered BNB events represents the event rate when the neutrino does not interact in the detector. The cosmic and neutrino likelihood functions absolutely normalized to $4 \times 10^{19}$ POT, then subsequently normalized by area are shown in Fig. 9.10.

Post pre-cut selection, both likelihoods show good agreement between data (black dots) and prediction across likelihood score value. Statistical errors only are shown per bin. The predicted neutrino content of the selection is broken down in the CCQE, MEC, inclusive $\pi^{ \pm}$ and $\pi^{0}$, and other charge current resonant events. In the low likelihood score the cosmic ray contribution dominates. The selection becomes increasingly rich in CCQE content as the likelihood is increased. No significant shape deviation is observed between the prediction and data.

After the two likelihood selection is applied, the sample of $1 \mu 1 \mathrm{P}$ candidates the becomes CCQE enriched. The reconstructed angle with respect to the beam for the identified muon and proton particles are shown in Fig. 9.11. 

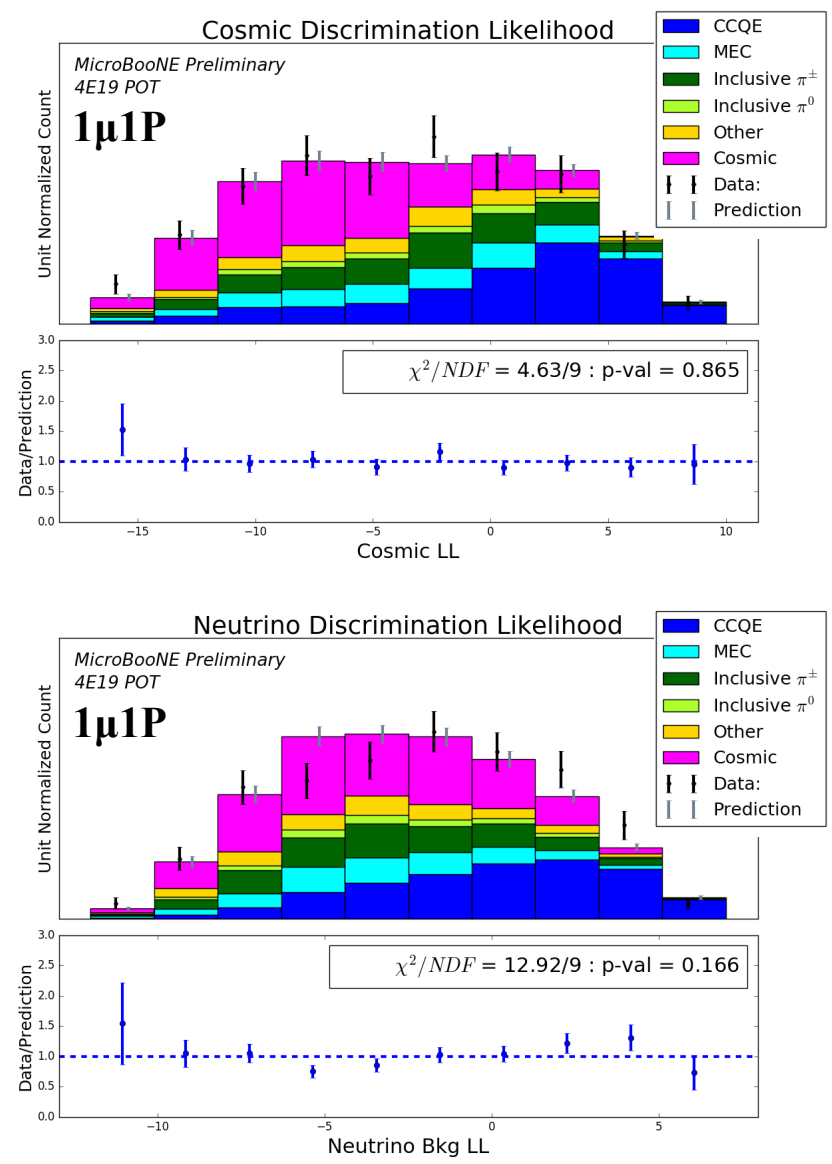

Figure 9.10: Cosmic (top) and Neutrino (bottom) LL score distribution absolutely normalized to $4 \times 10^{19} \mathrm{POT}$ for the $1 \mu 1 \mathrm{P}$ selection. The data and prediction are shown with black and gray dots respectively with statistical uncertainty only. The predicted likelihood distributions are broken down by neutrino interaction type. The ratio of data to prediction per bin is shown in the lower plot.

The proton is found to be primarily forward going with respect to the beam axis as expected for CCQE scattering. The muon particle features a range of kinematic angle. The sample is most pure with respect to cosmic ray events when the muon is forward going (above $\cos \theta_{\mu}>0$ ) as cosmic tracks do not feature two forward going particles. The cosmic contribution is the highest when the angle with respect to the beam is perpendicular indicating a downward going muon as expected. No significant shape deviation is observed between data and prediction in any of the reconstructed kinematic variables for the outgoing 

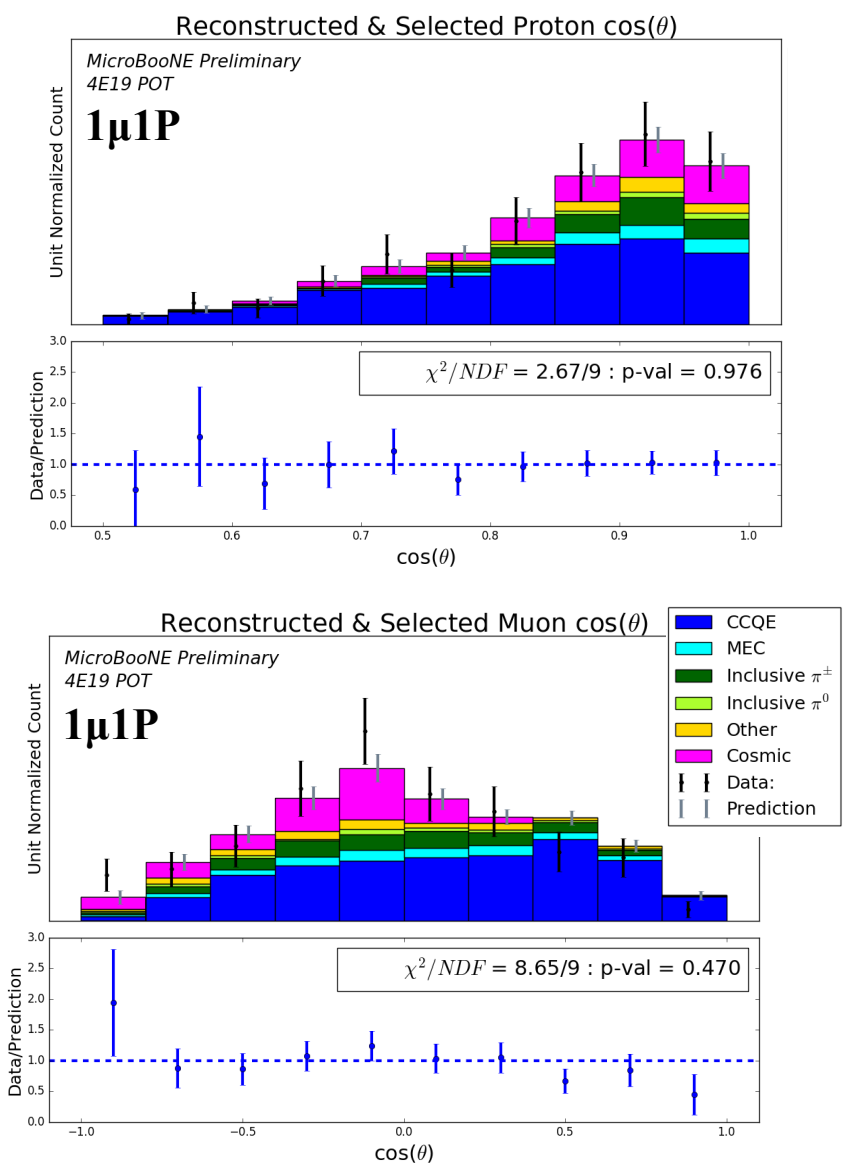

Figure 9.11: Proton (top) and Muon (bottom) angle with respect to the beam absolutely normalized to $4 \times 10^{19}$ POT for the $1 \mu 1 \mathrm{P}$ selection. The data and prediction are shown with black and gray dots respectively with statistical uncertainty only. The predicted $\cos \theta$ distributions are broken down by neutrino interaction type. The ratio of data to prediction per bin is shown in the lower plot.

proton and muon.

The reconstructed neutrino energy spectrum using the range based formula described in the previous section is shown in Fig. 9.12.

The sample is enriched in CCQE above $600 \mathrm{MeV}$ in reconstructed neutrino energy as the proton and muon particles can be optimally reconstructed. A total of 178 events are predicted while 163 are observed in data. The number of reconstructed muon neutrino events is sufficient at this number of POT to be used as a contraint of electron neutrino 


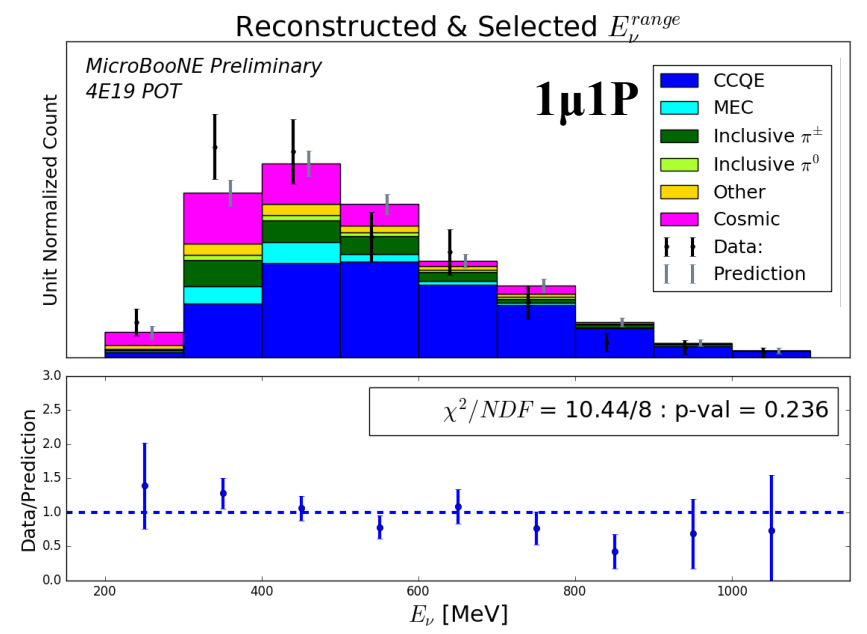

Figure 9.12: Reconstructed neutrino energy from the range based formula absolutely normalized to $4 \times 10^{19} \mathrm{POT}$ for the $1 \mu 1 \mathrm{P}$ selection. The data and prediction are shown with black and gray dots respectively with statistical uncertainty only. The predicted range based energy distribution is broken down by neutrino interaction type. The ratio of data to prediction per bin is shown in the lower plot.

systematic uncertainty.

To quantify the overall agreement between data and prediction, a shape only chi-square is computed for 23 reconstructed variables. The distribution of p-value is flat from 0.01 to 0.99 indicating good global agreement between data and prediction.

\section{$9.21 e 1 P$ Selection}

\subsubsection{Overview}

A selection is developed to identify $1 e 1 \mathrm{P}$ events against cosmic ray and neutrino backgrounds. A three stage multivariate approach based on machine learning algorithms are used for signal discrimination after a series of square cuts are applied to reduce cosmic ray backgrounds. First, a BDT is trained to topologically identify electron particles using features identified using the collection plane image. A BDT for cosmic discrimination is also studied. Second, an SVM is developed using 3D tracker kinematics to isolate a two particle topology. Finally, the Multi-PID network is leveraged via an SVM. The three scores are 
combined into a single discriminant for $1 e 1 \mathrm{P}$ selection.

The complexity of the $1 e 1 \mathrm{P}$ selection is high as identifying low energy electron neutrinos presents unique challenges not present in the muon neutrino selection. Due to the BNB flux, which is primarily of muon neutrino type, approximately 1 in 200 neutrino events is expected to be an electron neutrino interaction. As such, the expected number of cosmic ray only events is much larger. Topologically, low energy $1 e 1 \mathrm{P}$ events feature an electron shower whose feature is stochastic in nature and varies as a function of energy which increases the complexity of pattern recognition. Additionally, due to the expected size of the proton particle particle ID using the $d E / d x$ method must accurately characterize the charge deposition over a restricted number of wires depending on the projection. Two muon neutrino backgrounds are important in the $1 e 1 \mathrm{p}$ search. First, neutral current $\pi^{0}$ interactions featuring a single proton and two photons can fake a $1 e 1 \mathrm{P}$ signal when a single photon converts immediately at the vertex. The event rate for this topology is comparable to $1 e 1 \mathrm{P}$ interactions. Finally, low energy muons from $1 e 1 \mathrm{P}$ interactions feature a short trajectory and may generate radiation via delta rays which can topologically mimic an electron particle.

The selection is benchmarked on a Monte Carlo generated sample of BNB interactions equivalent to $6.6 \times 10^{20}$ POT to characterize the neutrino backgrounds. The same cocktail sample is used as in Section 9.1. An intrinsic electron sample, corresponding to $1 \times 10^{22}$ POT, features a GENIE generated neutrino interaction with data cosmic ray overlay and is used to estimate the expected electron neutrino contribution to the MC prediction. The

event rate prediction is compared to the $5 \times 10^{19}$ POT data set in reconstructed neutrino energy and pattern recognition variables to asses the Data-MC agreement.

\subsubsection{Topological BDT}

A topological selection based on the reconstruction outlined in Section 8.8 is performed. The reconstruction applies dedicated image based pattern recognition algorithms inside a restricted crop around the candidate vertex. First a series of cosmic ray rejection cuts are applied to candidate $1 e 1 \mathrm{P}$ vertices using the $2 \mathrm{D}$ cosmic ray tagging algorithm. Next, a cut on the number of particles exiting the vertex is applied and the electron and proton are 
identified. Finally topological features are identified and a BDT is trained for $1 \mathrm{e} 1 \mathrm{P}$ selection.

\section{Cosmic Rejection}

Three classes of cosmic ray interactions which appear as background to the $1 \mathrm{e} 1 \mathrm{P}$ search interact in some way with the candidate reconstructed vertex at the center of the crop. A visual aid is shown in Fig. 9.13. To reject these backgrounds, a set of square cuts is applied based on the proximity of tagged cosmic rays to the candidate vertex.
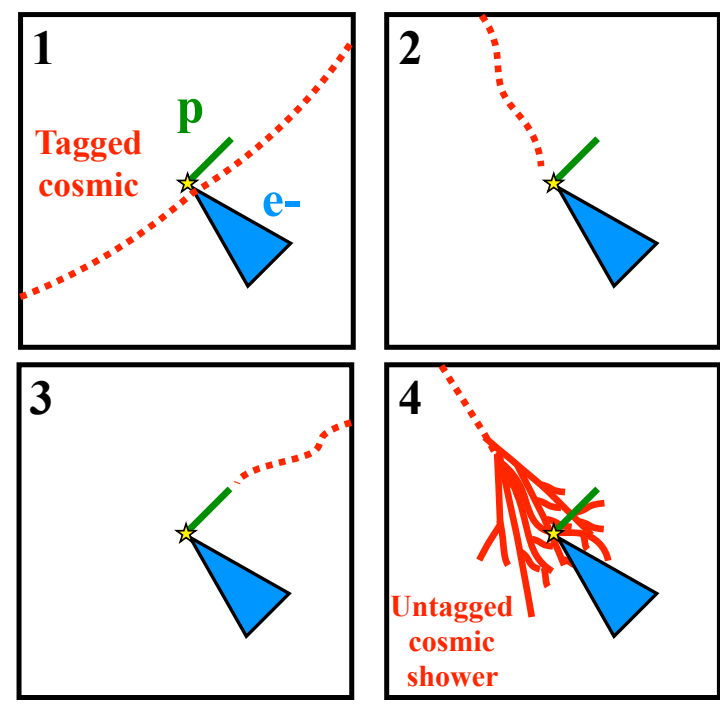

Figure 9.13: Four cosmic ray rejection precuts described in the text for topologically rejecting cosmic ray muons at the vertex location.

1. Through-going cosmic: Cosmic rays which travel through the image and approach within 2 centimeters of the candidate vertex are rejected. The clustering algorithm used to identify two prongs coming out of the vertex necessitates a clean search region. A nearby cosmic may leave some debris near the candidate vertex and may be misreconstructed.

2. Stopping cosmic: Cosmic rays which enter from the image edge may stop in the center via a michel electron decay. Cosmic rays which have a reconstructed end point within $6 \mathrm{~cm}$ of the candidate vertex are rejected. Additionally, the candidate vertex 
is rejected if either of the reconstructed particle end points is within $6 \mathrm{~cm}$ of a cosmic ray end point. This cut is reversed to select michel electron events and described in Section B.1.

3. Cosmic shower: High energy cosmic rays which enter into the image may produce appreciable energy loss due to bremsstrahlung photons. These cosmic showers produce a dense collection of charge and can cause a high degree of mis-reconstruction by the track-shower vertexing algorithm. If the candidate vertex is within a region of dense charge above a threshold, the vertex is rejected.

\section{Particle ID}

The candidate vertex is required to have exactly two particles reconstructed. The distribution of number of reconstructed particles is shown in Fig. 9.14. This reduces the cosmic ray and neutrino background by approximately $75 \%$ and $60 \%$ respectively while retaining approximately $80 \%$ of $1 e 1 \mathrm{P}$ events. Approximately $20 \%$ of $1 e 1 \mathrm{P}$ events are reconstructed with 3 particles and feature the presence of an additional proton below $60 \mathrm{MeV}$. These events are produced with MEC interactions.

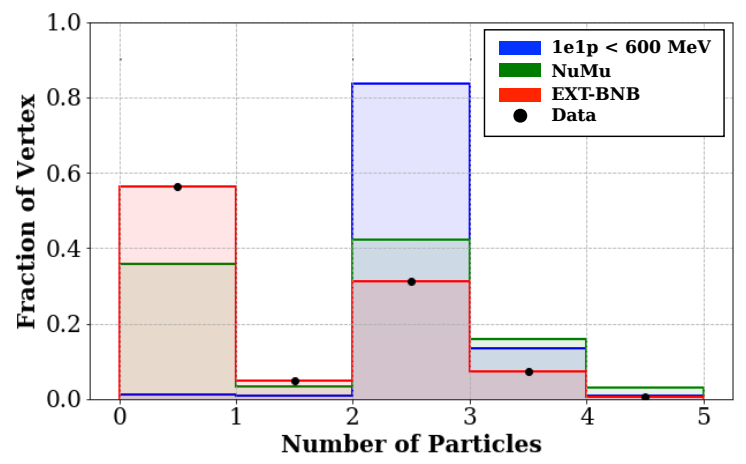

Figure 9.14: Number of particles reconstructed using the $1 e 1 \mathrm{P}$ algorithm for four samples.

The difference between the proton and electron topological feature is used to set the particle ID for the candidate vertex. For each reconstructed particle a line hypothesis is drawn on the image from the vertex to the particle end point. The fraction of the particle cluster which is contained inside the line hypothesis is called the line fraction. The particle with the largest line fraction is labelled as the proton particle, the other the electron. The 
distribution of line fraction for electron and proton particles is shown in Fig. 9.15
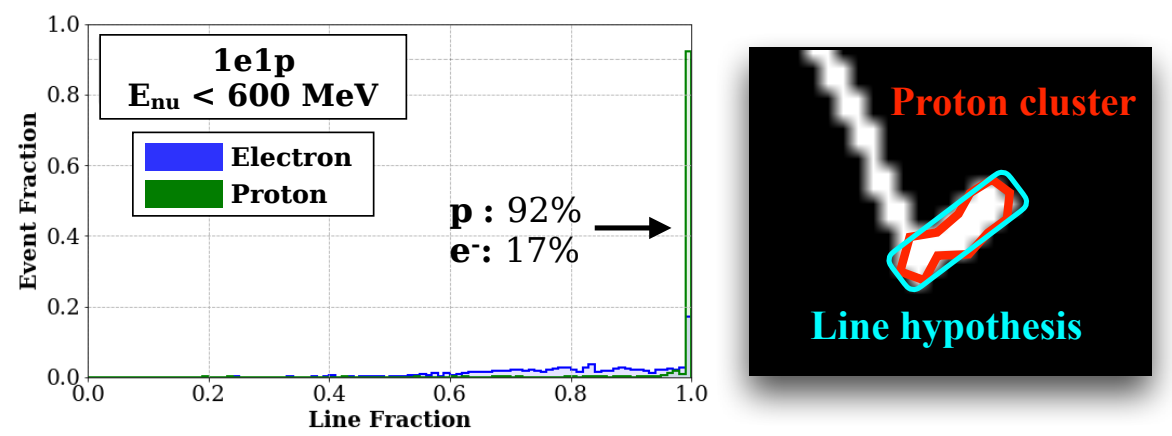

Figure 9.15: Left: Distribution of line fraction for true electron and proton particles for sub $600 \mathrm{MeV} 1 e 1 \mathrm{P}$ interactions. Right: Example estimation of the line fraction by drawing a line of fixed thickness (cyan) for a proton cluster (red).

Approximately $85 \%$ of $1 e 1 \mathrm{P}$ interactions feature a proton particle which is more straight than the corresponding electron. In some cases, the proton particle can scatter once, deviating from a straight line, or overlap with an additional low energy proton in the plane projection. It is also possible for the electron particle to have no shower feature and propagate with a straight line trajectory. Finally, a minimum calorimetric energy cut is applied to both the electron and proton particle of $60 \mathrm{MeV}$. The calorimetric energy is estimated by summing the charge of the collection plane particle cluster and is converted to $\mathrm{MeV}$ scale as described in Section. 8.8.5,

\section{Topological Features}

Electron and proton particles have unique features in a LArTPC image which can be used jointly to discriminate against backgrounds. Features identified using the $1 \mathrm{e} 1 \mathrm{P}$ algorithm from particle clusters are reconstructed on only the collection plane, due top poor agreement between data and simulation for features reconstructed on induction plane due to poor modelling of the signal shape. The selected features have been shown to have good agreement between data and MC and have been used successfully in traditional cut and likelihood based selection to isolate $1 e 1 \mathrm{P}$ events.

Eight features are used for proton particle identification. Discriminants are primarily 
used to distinguish proton particles against muons reconstructed on cosmic ray backgrounds. Profiles of select distributions are shown.

- Line Fraction The line fraction variable encodes the line like feature exhibited by low energy proton tracks. The distribution of line fraction is shown in Fig. 9.15. Particles which are perfectly straight in the projection will have a line fraction of 1.0 .

- Proton Kinematics For CCQE scattering the proton particle is expected to be forward going in the beam direction. The distribution of proton direction cosine in the $\mathrm{X}, \mathrm{Y}$, and $\mathrm{Z}$ are included as features for discrimination. To make the CCQE scattering requirement explicit, the fractional difference between the proton CCQE energy the reconstructed visible energy of the interaction is computed. The distribution of fractional difference is shown in Fig. 9.16. Finally, the 3D opening angle between the proton and electron is used to provide the strength of the vee shape topology.

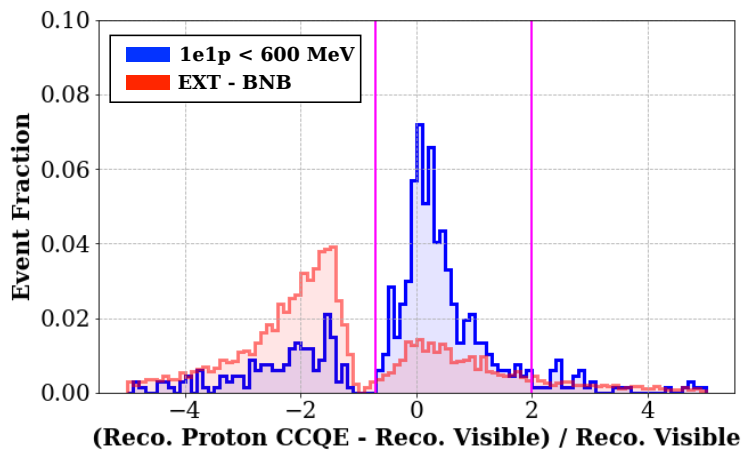

Figure 9.16: Distribution of fractional difference between the CCQE and visible energy of the interaction for $1 e 1 \mathrm{P}$ events (blue) and EXT-BNB events (red). Magenta lines represent the inner $68 \%$ of $1 e 1 \mathrm{P}$ events. $80 \%$ of EXT-BNB events lie outside the magenta region.

- Shower Fraction The likeliness that the selected identified proton particle is track like is quantified by counting the fraction of particle pixels on the collection plane which are shower like, called the shower fraction. A proton particle features a low shower fraction as the proton cluster is primarily labelled as track type. The distribution for proton and electron shower fraction is shown in Fig. 9.17. The SSNet classifies 
protons in low energy $1 e 1 \mathrm{P}$ events primarily of track type approximately $99 \%$ of the time, providing a useful discriminant against two track backgrounds.
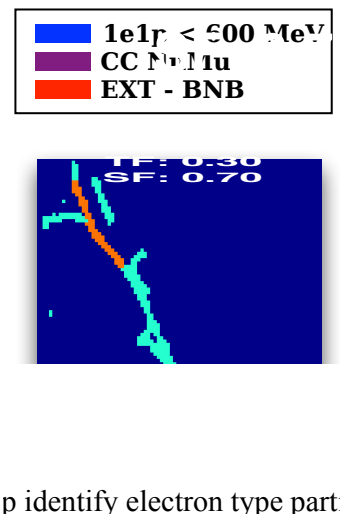

Orange $=$ track pixels Cyan $=$ shower pixels
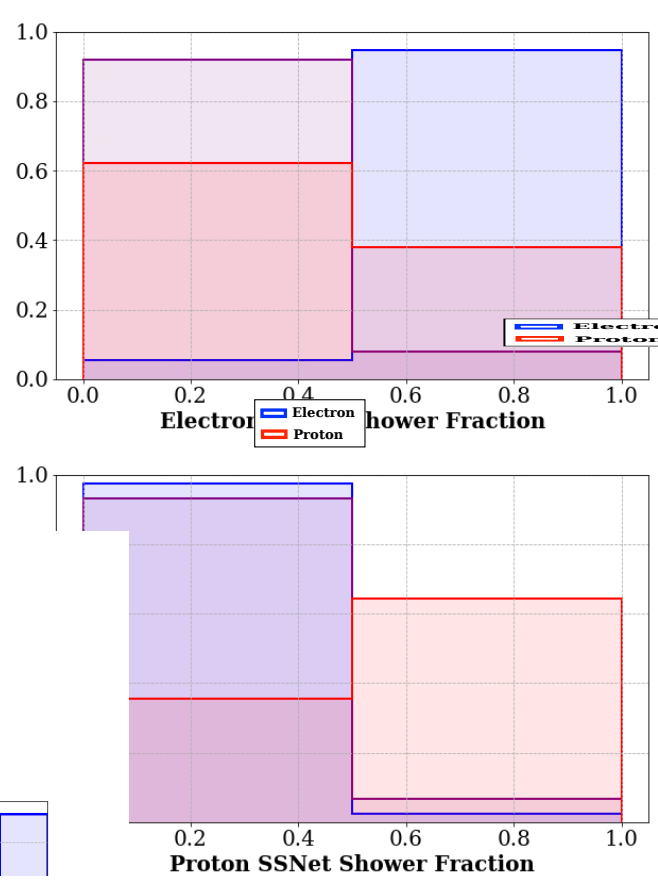

Figure 9.17: SSNet shower fraction for electron (top right) and proton (bottom right) particles reconstructed in $1 e 1 \mathrm{P}, \mathrm{CC} \nu \mu$, and EXT-BNB events. An example SSNet image of a $1 e 1 \mathrm{P}$ interactions is shown on the left where an electron particle features an SSNet shower fraction of 0.75 .

- $\mathbf{d E} / \mathbf{d x}$ The $d E / d x$ for the proton particle calculated as the median value over the full track length, as described in Section 8.8.6. The charge deposition per unit length is a valuable physics motivated discriminant to distinguish between short and straight proton particles from muons found on cosmic ray backgrounds.

- Proton Multi-PID Score. The Multi-PID network has been shown to perform well at identifying proton particles in simulated vertices in liquid argon. The proton score from the Multi-PID network is used a feature for background discrimination

The primary means to identify an electron particle are to identify average shower features and quantify the topological shape of the electromagnetic cascade. A subset of the 
extracted shower features are described in Section 8.8.3. These features are used as discriminants against low energy muon particles produced in BNB neutrino interactions. Shower characteristics are estimated using tweleve features for electron particle identification.

- Triangle Parameters The presence of an electron shower feature can be can be estimated via the ionization spread of the particle perpendicular to its propagation direction. The $1 e 1 \mathrm{P}$ algorithm provides a triangle optimized to fit the electromagnetic shower of the electron and is used to estimate the spread. The ratio of the shower length to its width quantifies the spread and is shown in Fig. 9.18, The ratio is related to the radiation length, and Moliere radius for electrons in liquid argon which are 14 $\mathrm{cm}$ and $9.042 \mathrm{~cm}$ respectively [6].
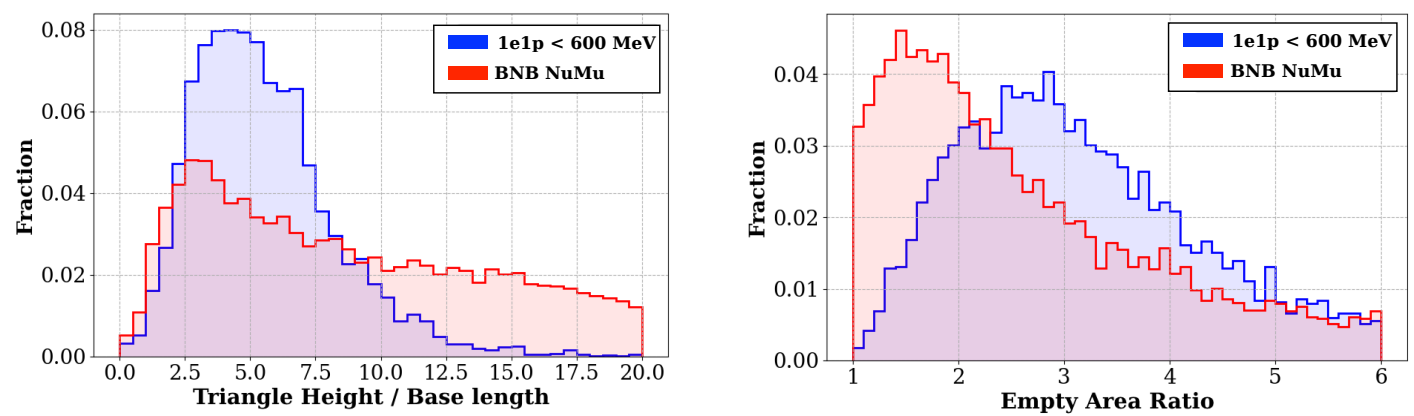

Figure 9.18: Left: Distribution of ratio of electron shower length to width for $1 e 1 \mathrm{P}$ events (blue) and neutrino backgrounds events (red). Right: Distribution of empty area ratio for electron particles for 1e1P events (blue) and neutrino background events (red).

- Empty Area Ratio To further capture the spread of an electron shower's electromagnetic cascade the ratio of the amount of empty area enclosed by the convex hull to the number of non-zero pixels inside is used, called the empty area ratio. The ratio captures the degree to which the radiation spreads to cover an area. The ratio is high if the cascade divides into smaller branches as it propagates outward and low if very little radiation is emitted, such as if a muon features some delta radiation. The metric is shown in Fig. 9.18. 
- Shower Structure The micro-structure of the shower is quantified using custom topological algorithms to examine the fine details of the electromagnetic cascade. The fraction of the shower cluster which deviates from a straight line measured after the showering point is used remove the bias of the electron trunk which is typically straight. The branching structure of the electron is quantified by counting the number of edges and branches which compose the cascade. Branches are defined as points on the cascade which form a "Y" shape where a single line of charge deposition breaks into two or more prongs. The edges are the end points of branches which manage to terminate off the central core of the shower. To further characterize the shower, the number of defect points using a convex hull approximation for the shower is used to further asses the branching structure. The distribution of branches and defects are shown in Fig. 9.19, Finally, the shower fraction as described above is used to count the number of pixels labelled as shower type by the SSNet.
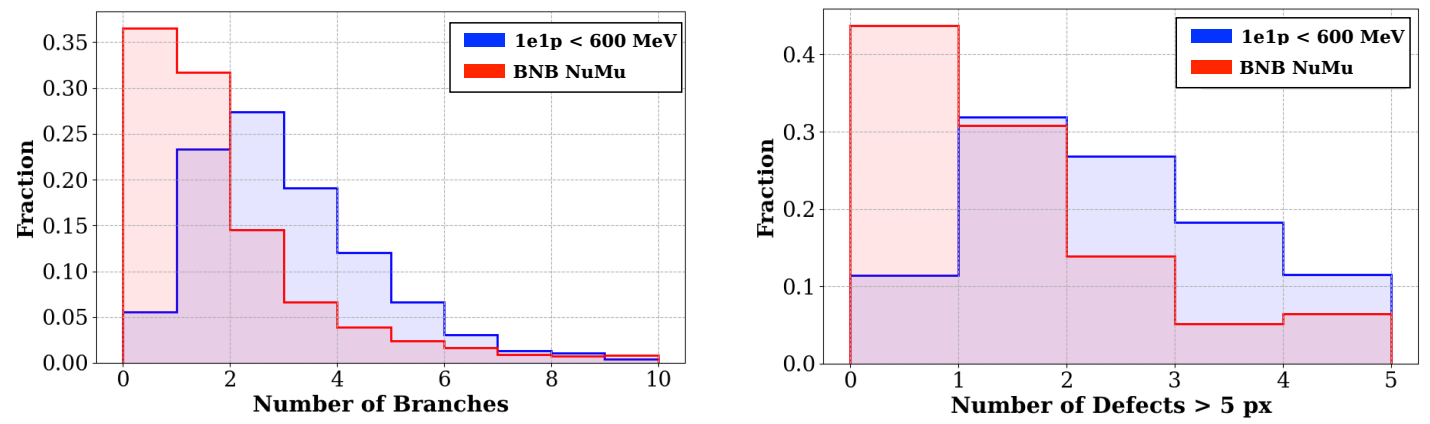

Figure 9.19: Discriminators used to identify electron topologies described in the text. Left: Distribution of number of branches for electron particles identified in $1 e 1 \mathrm{P}$ (blue) and neutrino background (red) events. Right: Distribution of number of defect points larger than 5 pixels.

- Detached Clusters A unique feature of electron particles above approximately 30 $\mathrm{MeV}$ is the emission of radiated photons. Radiative photons are clusters of charge emitted in the direction of electromagnetic shower and are detached from the primary electron shower. The number of detached clusters above $25 \mathrm{MeV}$ is counted and used 
for electron versus low energy muon discrimination.

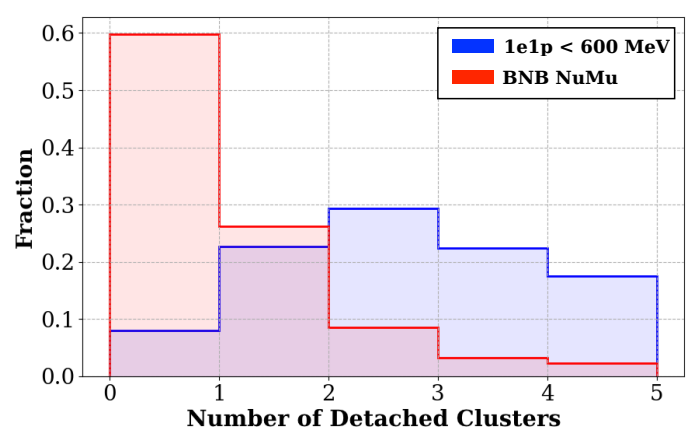

Figure 9.20: Number of identified clusters emitted in the electron direction above $25 \mathrm{MeV}$ for $1 e 1 \mathrm{P}$ (blue) and neutrino background (red) events.

Nineteen combined proton and electron features are used for classification of $1 \mathrm{e} 1 \mathrm{P}$ events against cosmic and neutrino backgrounds described in the following section.

\section{BDT Selection and Efficiency}

Two BDTs are trained to distinguish $1 e 1 \mathrm{P}$ interactions against cosmic ray (Cosmic BDT) and neutrino (Neutrino BDT) backgrounds. A gradient boosted decision tree framework called XGBoost [43] is used to train a decision tree ensemble which consists of a set of classification trees. A decision tree is analogous to a series of sequential rectangular cuts on features used to discriminate two populations such signal and background high energy physics processes. Instead of identifying the relevant features and hand designing the selection value, a machine learned algorithm is used to build a collection of trees with varying number of leafs, or subsequent cuts. Each leaf is analogous to an additional selection cut on a subsequent feature to separate signal and background populations. During the training process a new tree is added, called boosting, during each training iteration to correct the errors mades by the previous sequence of trees. Each tree outputs a prediction score and the result is summed across trees to determine the final classification. The result is a score for being either part of a signal or background population.

The cosmic and neutrino BDTs are trained using 50 trees with a maximum depth of 3 leafs using a softmax objective. The signal sample is prepared from 5000 well reconstructed 
$1 e 1 \mathrm{P}$ interactions with good truth matching between simulated and reconstructed particles. Training and validation samples are randomly split $80 \% / 20 \%$. True neutrino energy range is flat between 0 and $1 \mathrm{GeV}$ with $70 \%$ of events being below $500 \mathrm{MeV}$ to enrich the LEE signal sample. Background samples are prepared as follows:

- Cosmic BDT: 5000 reconstructed cosmic ray events from the EXT-BNB sample which pass the cosmic ray rejection cuts outlined in the previous section are used as the background class.

- Neutrino BDT: 5000 well reconstructed BNB intrinsic $\nu_{\mu}$ events of various interaction channel which have a well reconstructed muon and proton particle.

The network is trained to approximately equivalent train and test accuracy. The results of applying the networks on the respective background samples are shown in Fig. 9.21. Using a score cut of 0.5 , where a value larger than 0.5 is deemed "signal", $85 \%$ of $1 e 1 \mathrm{P}$
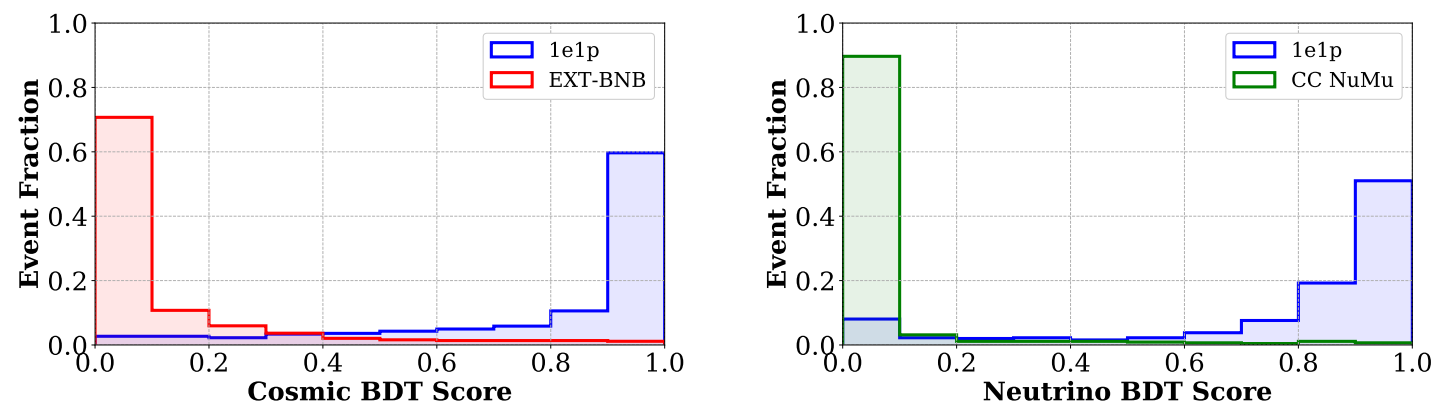

Figure 9.21: Score distributions for Cosmic BDT (left) and Neutrino BDT (right) for signal and background inputs.

and $93 \%$ of EXT-BNB testing events are classified correctly via the Cosmic BDT. For the Neutrino BDT, $84 \%$ of $1 e 1 \mathrm{P}$ and $96 \%$ of neutrino backgrounds are classified as correct. To determine the relative importance of each feature the number of times a feature appears in the ensemble as a discriminant is shown in Fig. 9.22 .

For discriminating proton particles the BDT favors the Multi-PID proton score, the $\mathrm{dE} / \mathrm{dx}$, and the angle of the proton as strong features for separation from cosmic backgrounds. The Neutrino BDT favors topological parameters using the particle length, size 

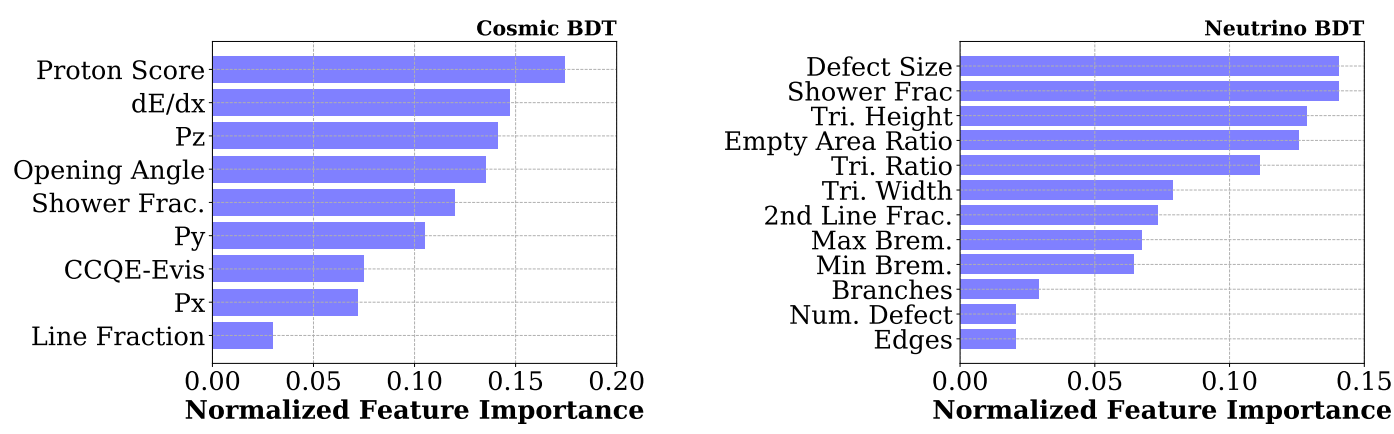

Figure 9.22: Importance of features used in the Cosmic (left) and Neutrino (right) BDTs. Feature importance is a normalized measure of the fraction of algorithm composed of the specified discriminant.

of defects present in the shower, and the number of pixels labelled as shower type by the SSNet.

The Cosmic and Neutrino BDT are applied to events after cosmic ray rejection cuts to estimate the score distribution for $5 \times 10^{19} \mathrm{POT}$ data. For each events the two BDTs are applied. To prevent bias, the candidate vertex with the lowest flash matching score is chosen per event. The absolutely normalized score distribution is shown in Fig. 9.23.
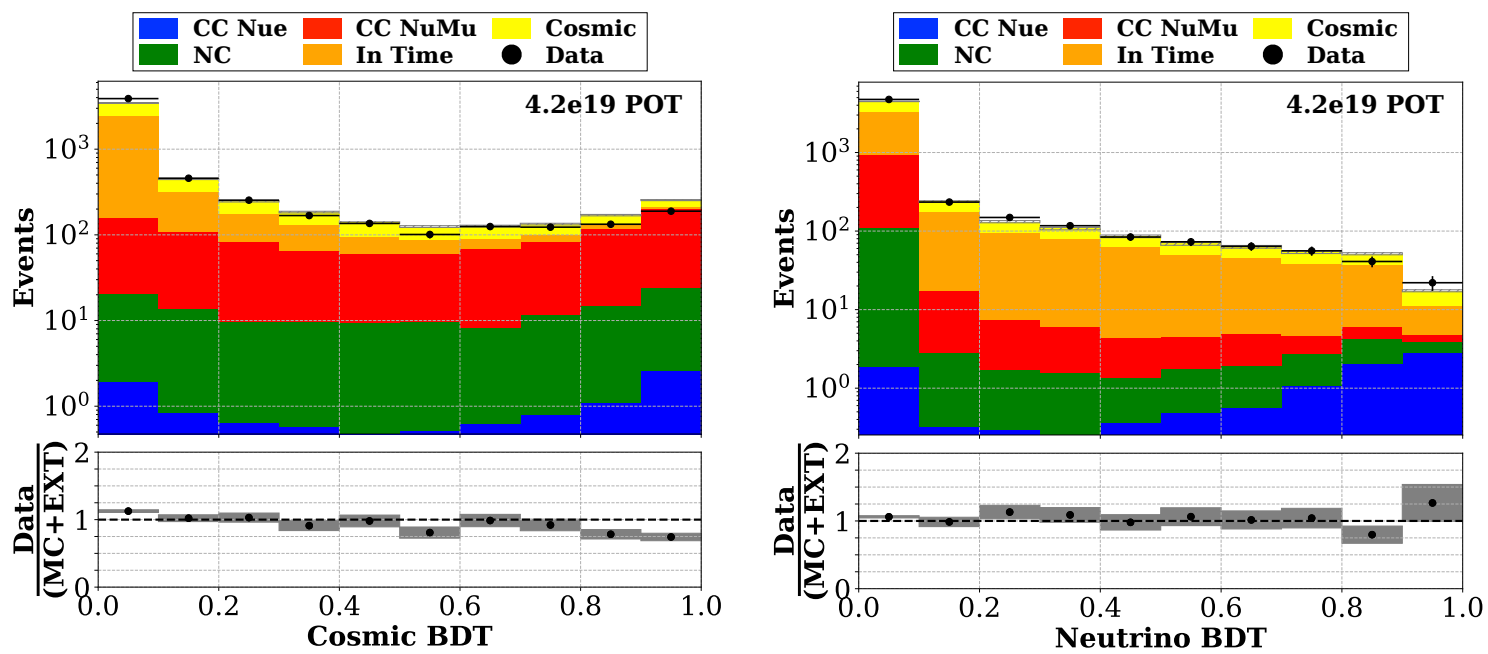

Figure 9.23: Event rate prediction for Cosmic (right) and Neutrino (left) BDT for backgrounds for the $1 e 1 \mathrm{P}$ selection described in the text. Run 1 data are shown as the black dot. 
Five contributions to the event rate are shown, three neutrino induced and two cosmic induced distributions. The event rate for neutrino induced interactions consists of well reconstructed CC Nue (blue), neutral current (NC), and CC NuMu (red interactions). The cosmic event rate is comprised of an "In Time" contribution (orange) which represents events in time with the beam but no neutrino interaction present in the event, and a "Cosmic" contribution which represents an event where a cosmic ray is selected in an event with a neutrino. Good agreement is observed between data and prediction for both BDTs. The neutrino enriched regions (low and high) of the Cosmic BDT approximately $25 \%$ disagreement is observed in the rate. At cut on the score distributions is optimized by considering the ratio of signal $(1 e 1 \mathrm{P})$ to other backgrounds. The rate prediction as a function of reconstructed energy for an optimized score cut of 0.70 on both the Cosmic and Neutrino BDT is shown in Fig. 9.24.

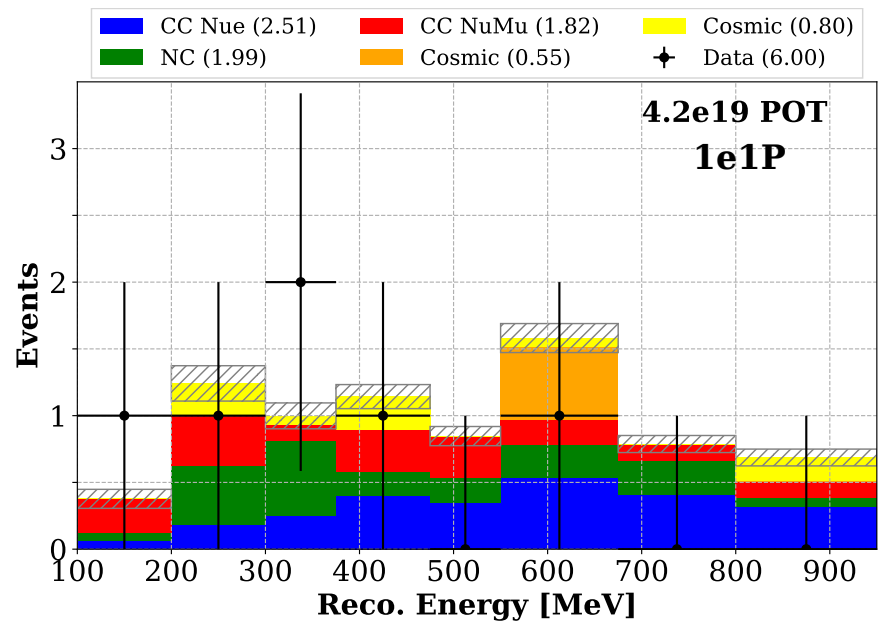

Figure 9.24: Event rate prediction as a function of reconstructed neutrino energy for an optimized BDT score cut of 0.70 for the $1 e 1 \mathrm{P}$ selection. In data $6.0 \pm 2.4$ events are observed with a prediction of $7.3 \pm 0.5$ (stat). Only statistical error bars are shown (gray dash).

After selection, two primary neutrino backgrounds dominate. First, neutral current $\pi^{0}$ events which produce a proton and two photons in which one photon converts immediately at the vertex. This topology can fake a $1 e 1 \mathrm{P}$ interaction. In this selection no algorithm is used to identify the presence of a correlated second shower detached from the vertex point. Second, low energy $\mathrm{CC} \nu_{\mu}$ events feature a short muon which produces delta radiation 
comparable in size to the muon length. These events visually mimic low energy showers as they feature a branching structure after a straight trunk region. From a handscan of the data events two 1e1P candidates are observed and shown in Fig. 9.25 and Fig. 9.26.

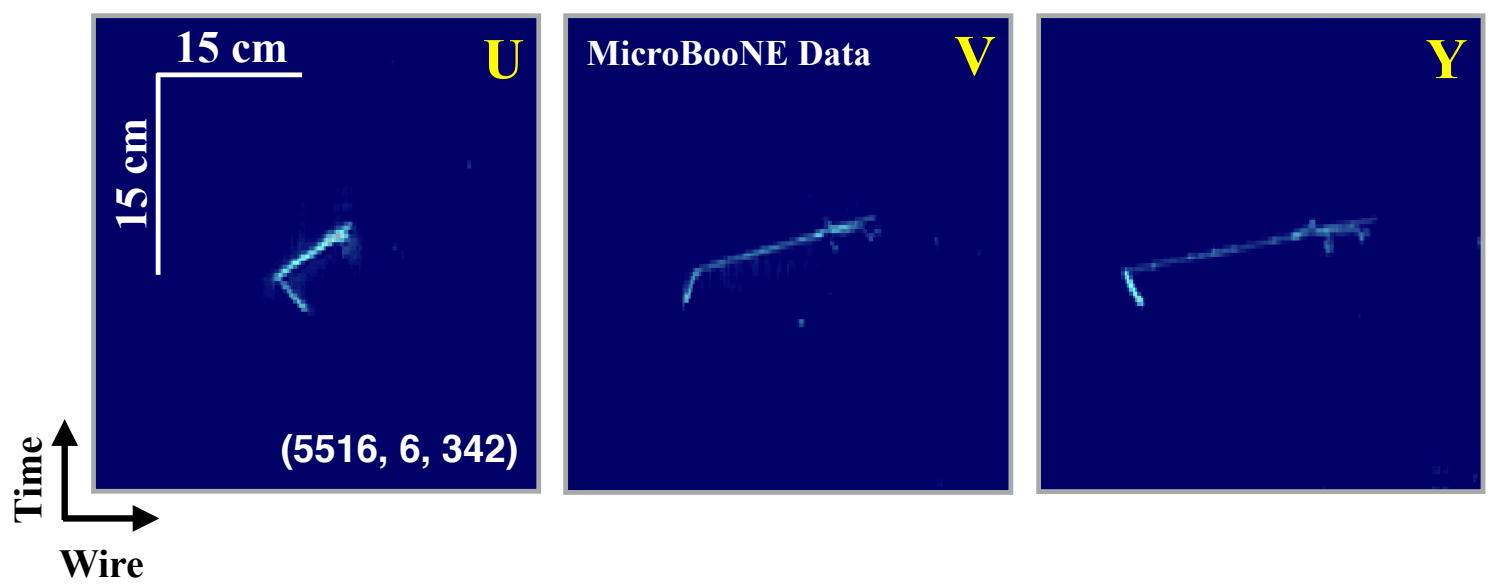

Figure 9.25: Reconstructed $1 e 1 \mathrm{P}$ candidate interaction identified in the Run 1 data set. The reconstructed energy is $166 \mathrm{MeV}$.

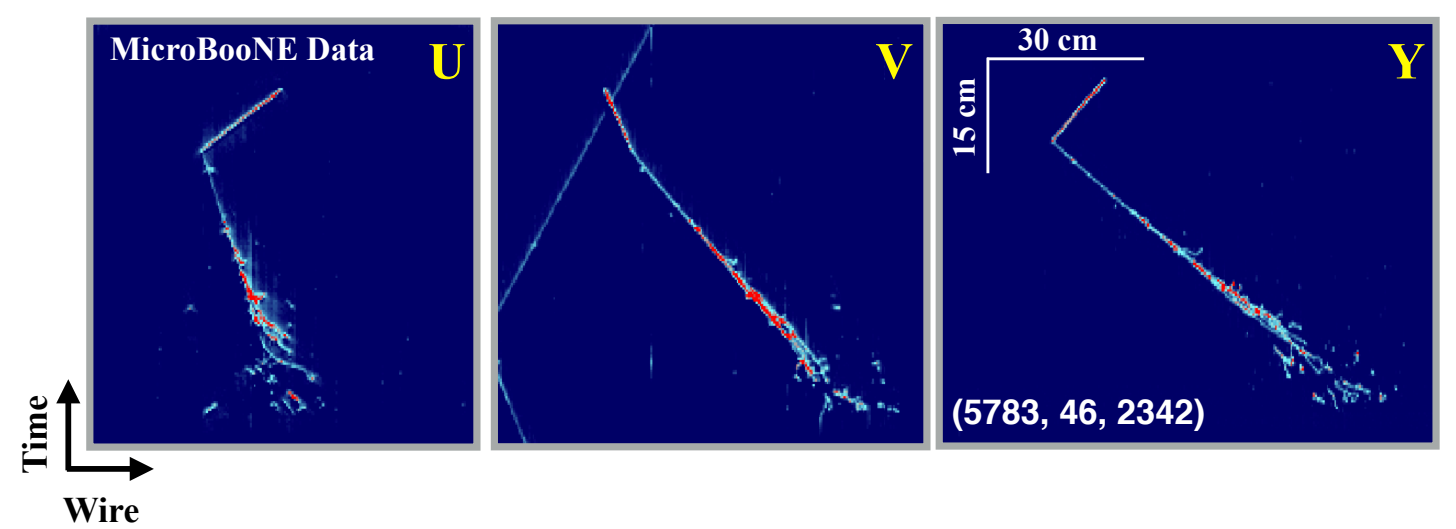

Figure 9.26: Reconstructed $1 e 1 \mathrm{P}$ candidate interaction identified in the Run 1 data set. The reconstructed energy is $1.1 \mathrm{GeV}$.

The efficiency of the BDT based selection is shown as a function of true neutrino energy in Fig. 9.27 after applying the cosmic ray rejection cuts, and the two BDT classifiers. After the final Neutrino BDT cut is applied, the $1 e 1 \mathrm{P}$ efficiency increases from approximately $10 \%$ in the lowest energy bin to $20 \%$ across the low energy excess region. 


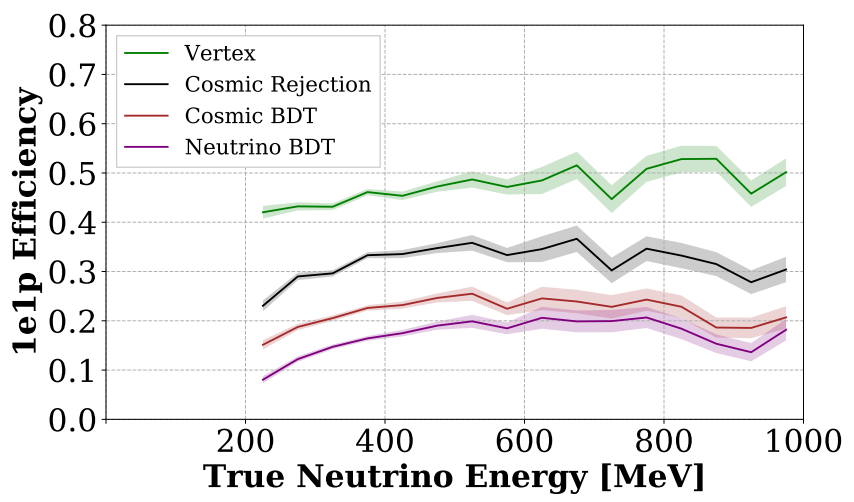

Figure 9.27: 1e1P efficiency as a function of true neutrino energy for 3 analysis cuts in the BDT based selection. The top line (green) is the cumulative vertex efficiency before selection cuts and represents the nominal output of the vertexing algorithm with backgrounds shown in Fig. 9.1. The black, maroon, and purple lines represent the cosmic rejection cuts, and the Cosmic and Neutrino BDT cuts respectively. The stacked backgrounds compared to $5 \times 10^{19}$ POT data are shown in Fig. 9.24 after the Neutrino BDT cut. 


\subsubsection{Kinematic \& Multi-PID SVMs}

To fully exploit the available feature space the 3D tracking and Multi-PID algorithms are used for 1e1P selection using two support vector machines (SVM) algorithms. An SVM is a supervised learning algorithm for classification and regression problems. In this analysis, and SVM is trained to map a set of hand-designed features to a classification score as either signal or background. The SVM is an optimized hyper plane in the number of features dimension found by minimizing a hinge loss function which determines the optimal margin between the hyper plane and the support vectors.

\section{Kinematic SVM}

The Kinematic SVM is a $1 e 1 \mathrm{P}$ vs. CC $\nu_{\mu}$ classifier built from features extracted from the $3 \mathrm{D}$ track reconstruction algo. While not specifically designed for reconstructing electron showers, the tracking algorithm can provide useful information about an electron showers trajectory. The tracker can identify the presence of a straight trunk region followed by a zigzagging trajectory as the tracker attempts to follow the pattern shower-like. As in the case of constructing the Topological BDT as described in Section 9.2.2, a set precuts are applied to reject obvious cosmic ray and muon neutrino background features. A cut is placed on the CCQE energy consistency as described in Section 9.1 to ensure good agreement of the proton and electron particle with CCQE kinematics. Additionally, two cuts are used to reject charge and neutral current $\nu_{\mu}$ interactions. First, a muon identification algorithm detects the presence of a michel electron located at the end of a track. These events are typically $\mathrm{CC} \nu_{\mu}$ events where a vertex is found at the true scattering point as well as the michel decay point. Finally, an algorithm is used to identify the presence of a second correlated shower with the neutrino vertex point. If a large $\left(9 \mathrm{~cm}^{2}\right)$ shower is identified with a larger than 45 degrees opening angle with respect to the attached shower the candidate vertex is vetoed.

Next, the SVM is trained using five features described in Section 9.1 ratio of the transverse momentum to the total momentum, Bjorken $\mathrm{x}, 3 \mathrm{D}$ opening angle, the $d Q / d x$ asymmetry $\eta$, and the CCQE energy consistency cut. The result is shown in Fig. 9.28. Separation is observed between $1 e 1 \mathrm{P}$ and $\nu_{\mu}$ interactions. A score cut of 0.5 is used to de- 


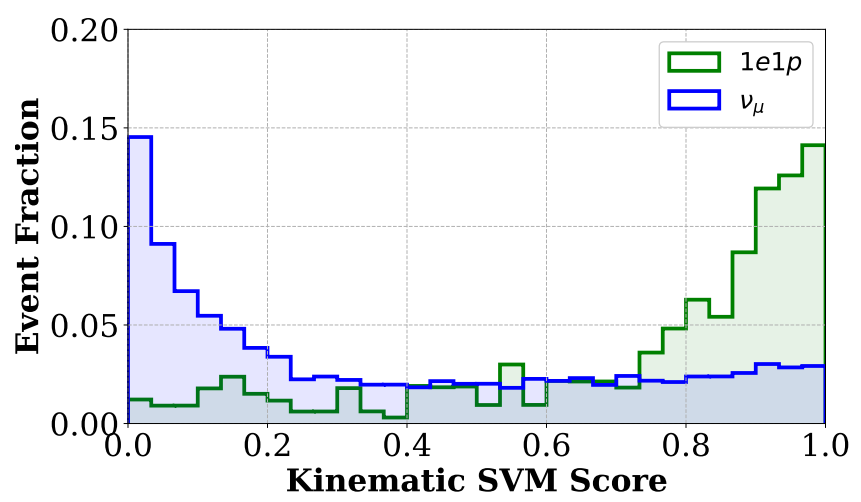

Figure 9.28: Kinematic SVM classifier score applied to $1 e 1 \mathrm{P}$ (green) and $\nu_{\mu}$ (blue) background events.

termine the classification as signal. Approximately $81 \%$ of $1 e 1 \mathrm{P}$ and $35 \%$ of $\nu_{\mu}$ interactions are classified as signal.

\section{Multi-PID SVM}

An SVM is trained using the output of the Multi-PID network to distinguish 1e1P events against $\nu_{\mu}$ backgrounds. Three features are used to distinguish the presence of a proton, muon, and shower type particle. A shower type particle is identified in the interaction by taking the max score between the electron and photon score. Good agreement is observed in rate and shape for applying the Multi-PID network to predict the Run 1 data set. The results are shown in Fig. 9.29

The result of training the Multi-PID SVM as applied to a sample of $1 e 1 \mathrm{P}$ and $\nu_{\mu}$ background events is shown in Fig. 9.30. Separation is observed between $1 e 1 \mathrm{P}$ and $\nu_{\mu}$ interactions. A score cut of 0.5 is used to determine the classification as signal. Approximately $93 \%$ of $1 e 1 \mathrm{P}$ and $7 \%$ of $\nu_{\mu}$ interactions are classified as signal. 

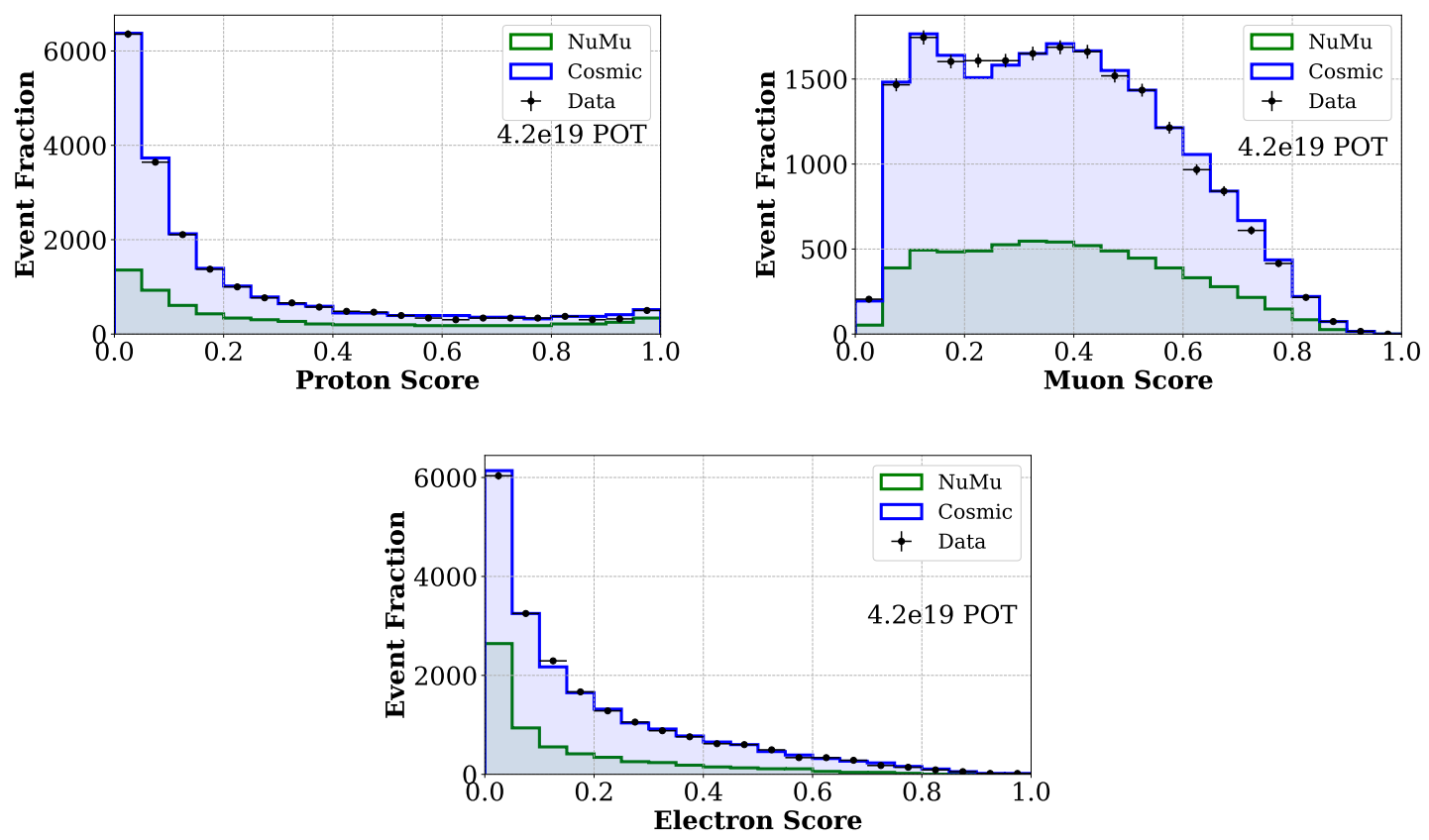

Figure 9.29: Proton (upper left), muon (upper right), and electron (lower middle) score distributions. The blue and green stacked histograms are the Cosmic and $\nu_{\mu}$ backgrounds respectively. The black dots are the score distributions for the Run 1 data set. Good agreement is observed between data and prediction.

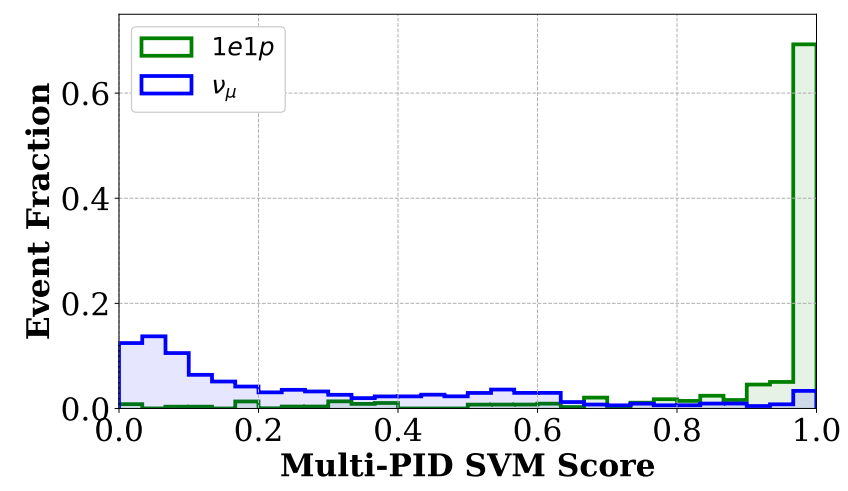

Figure 9.30: Multi-PID SVM classifier applied to $1 e 1 \mathrm{P}$ (green) and $\nu_{\mu}$ (blue) events. 


\subsubsection{Combined Selection}

The output of the three machine learned algorithms is combined into a single score classifier by taking an average. The classifier is shown in Fig. 9.31.

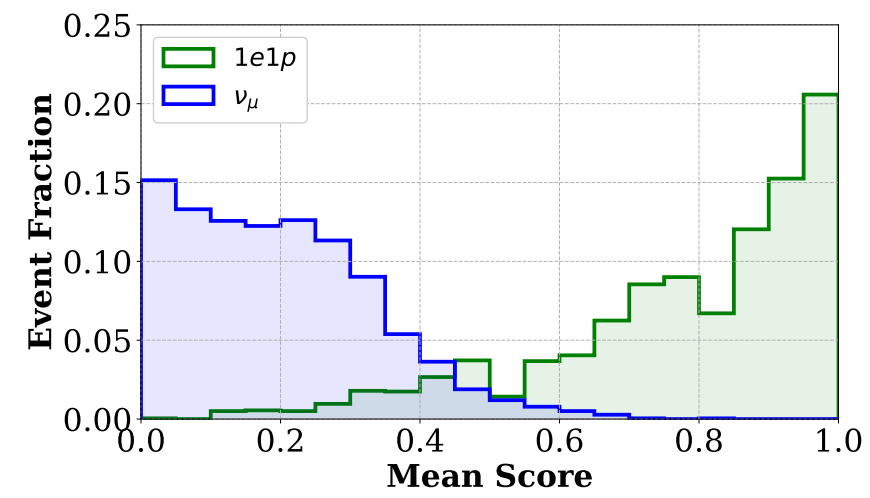

Figure 9.31: Combined classifier for $1 e 1 \mathrm{P}$ selection using the Topological BDT, the Kinematic SVM, and the Multi-PID SVM. Two distributions are shown: 1e1P (green) and $\nu_{\mu}$ (blue) interactions.

A cut is placed at a mean score value of 0.65 to remove all intrinsic $\nu_{\mu}$ backgrounds. The reconstructed energy is calculated by using the range based energy for the proton and calorimetric energy of the electron particle. The energy distribution for intrinsic $\nu_{e}$ events after the combined classifier is shown in Fig. 9.32 scaled to $13.2 \times 10^{20}$ POT. Neither cosmic or intrinsic $\nu_{\mu}$ backgrounds are predicted in $13.2 \times 10^{20}$ POT data with an integrated $1 \mathrm{e} 1 \mathrm{P}$ efficiency of $\sim 5 \%$. 


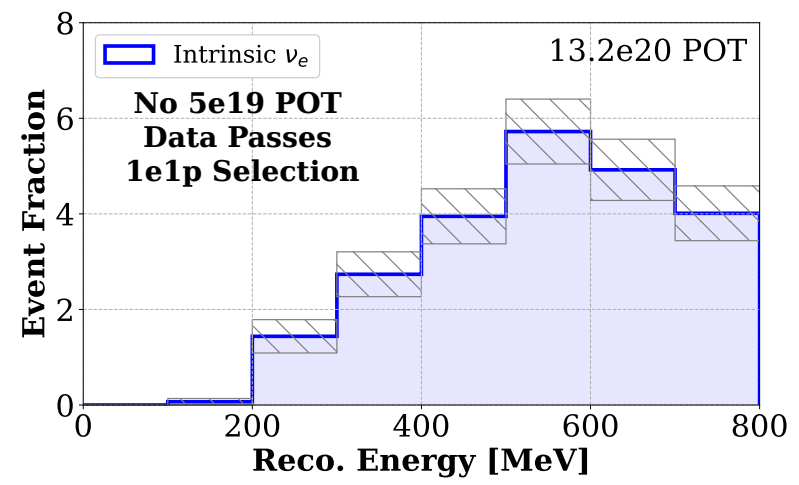

Figure 9.32: Reconstructed energy distribution for intrinsic $\nu_{e}$ events after the combined classifier. MC statistical error bars are shown. No other neutrino or cosmic backgrounds pass the combined selection. 


\section{Chapter 10}

\section{Systematic Errors}

This chapter describes the systematic errors associated with the LEE search. Propagation of flux, cross section, and detector modelling systematic uncertainties are discussed using the covariance matrix formalism. Finally, a demonstration of the $\nu_{\mu}$ constraint of the $\nu_{e}$ systematic uncertainties is presented.

\subsection{Overview}

Quantifying the significance of a low energy excess signal and assessing the sensitivity to $3+1$ neutrino oscillations depends on the accuracy and precision for measuring the neutrino flavor and energy spectrum. In MicroBooNE, the cross section uncertainties in the GENIE neutrino generator, the modelling of the beam flux, and the detector uncertainties contribute to neutrino measurement uncertainties. The covariance matrix formalism is used to propagate the systematic uncertainty from various sources. The covariance matrix itself is a structure which characterizes the systematic shift in an observable, in this thesis the reconstructed neutrino energy, which are correlated across bins. The covariance matrix is constructed by varying a systematic parameter, quantifying the change in the observable spectrum, and comparing the spectrum to the nominal, or central value. Specifically,

$$
M_{i j}=\frac{1}{n} \sum_{k}^{n}\left(N_{i}^{\mathrm{CV}}-N_{i}^{k}\right) \times\left(N_{j}^{\mathrm{CV}}-N_{j}^{k}\right),
$$


where $M_{i j}$ is the covariance matrix, $n$ is the number of "universes" for which the systematic is sampled, and $N_{i}^{\mathrm{CV}}$ and $N_{i}^{k}$ are the nominal and $k$ th variation at bin $i$. The total uncertainty is then a combination of independent matrices for each systematic source,

$$
M^{\text {Sys. }}=M^{\text {GENIE }}+M^{\text {Flux }}+M^{\text {Detector }} .
$$

GENIE and flux uncertainties are estimated using the multisim method [44]. In this approach, MC events are generated with model parameters set to their central value. Then, model parameters are drawn randomly for each parameter within their given uncertainty. For each sampled parameter set, the event is assigned a weight based on the ratio of the flux or cross section for the universe compared to the central value. Uncertainties on the model parameters are propagated to weights for an event, which in the end are used to evaluate the associated systematic uncertainties on the final event distributions using Equation 10.1. A caveat to this procedure is that event re-weighting does not vary the kinematics of the events which may be affected by certain cross section uncertainties. In this chapter, the systematic uncertainties associated with detector modelling are not included as dedicated detector variations are unavailable. A brief discussion is given in Section 10.4.

The procedure for constraining the $\nu_{e}$ systematic uncertainties using the $\nu_{\mu}$ spectrum is powerful technique to exploit flux and cross section correlations between the $\nu_{e}$ and $\nu_{\mu}$ energy bins. The constraint is detailed in Section 10.5 but referred to in Sections 10.2 and 10.3 ,

\subsection{GENIE Cross Section}

Neutrino interaction uncertainties are estimated using the event reweighing technique. A table of tunable neutrino-generator input physics quantities are shown in Table. 10.1 and include form factors for various types of neutrino-nucleus interactions, intra-nuclear hadron transport parameters in the final state interactions (FSI) model, and many more. Only some of the neutrino interaction uncertainties dominate. Weights for several different universes in which the varied parameter value is sampled from a gaussian distribution while all other parameters are held at their central value. The number of universes per parameter is between 50 and 1,000 depending on its importance. One shortcoming of the current ap- 
proach is that it is based on the default GENIE model for quasi-elastic interactions and an empirical model for MEC interactions. The total constrained $\nu_{e}$ neutrino interaction uncertainty is estimated to be approximately $10 \%$ and is dominated by FSI model uncertainties, and $\mathrm{CC}$ resonant and $\mathrm{NC}$ model parameters which are not well constrained. QE related cross section systematics, such as QE vector and axial mass, are well constrained and a reduced to less than $1 \%$ for each variation. For $\nu_{\mu}$ interactions, the neutrino interaction uncertainties total approximately $25 \%$.

\subsection{BNB Flux}

The BNB flux uncertainties are estimated for MicroBooNE using the beamline simulation and techniques developed by the MiniBooNE collaboration [14]. The flux uncertainties considered are,

- Hadron production of $\pi^{+}, \pi^{-}, K^{+}, K^{-}$, and $K_{L}^{0}$.

- Horn current mis-calibration, and mis-modeling of horn current distribution, such as the depth by which the current penetrates the horn conductor or so-called skin-effect.

- Secondary hadron interactions including $\pi^{ \pm}$and nucleon total, inelastic, and quasielastic scattering cross-sections on aluminum and beryllium.

In addition, there is an overall $2 \%$ normalization uncertainty due to uncertainties in proton delivery to the target. The multi-sim procedure is used by varying 13 flux parameters in 1,000 universes. The $1 \sigma$ uncertainty estimates for these parameters are the same as in the MiniBooNE paper, except for the $K^{+}$and $\pi^{ \pm}$production uncertainties, which have been improved by more recent measurements. The $K^{+}$production uncertainty has been constrained using the SciBooNE measurement of $p+\mathrm{Be} \rightarrow K^{+}$at the BNB [16] [45]. The $\pi^{ \pm}$ production uncertainties have been improved by using a better, more direct technique for extracting uncertainties from the HARP data than the original Sanford-Wang parameterization. These updates are both reflected in more recent MiniBooNE papers, such as in [46]. The total constrained $\nu_{e}$ flux uncertainties are estimated to be approximately $3 \%$ and are dominated by uncertainty associated with $\pi^{+}$production. 


\begin{tabular}{|c|c|}
\hline GENIE Reweight & Description \\
\hline MaNCEL & NC Elastic axial mass \\
\hline EtaNCEL & NC Elastic strange FF eta \\
\hline MaCCQE, VecCCQEshape & QE axial and vector mass \\
\hline MaCCRES, MvCCRES & $\mathrm{CC}$ resonance axial and vector mass \\
\hline MaNCRES, MvNCRES & $\mathrm{NC}$ resonance axial and vector mass \\
\hline MaCOHpi, R0COHpi & Coherent pion prod. axial mass, and nuclear size \\
\hline $\begin{array}{l}\text { RvpCC1pi, RvbarnCC1pi, } \\
\text { RvpNC1pi, RvbarnNC1pi }\end{array}$ & Non-res background, 1pi nu \\
\hline $\begin{array}{l}\text { RvnCC1pi, RvbarpCC1pi, } \\
\text { RvnNC1pi, RvbarpNC1pi }\end{array}$ & Non-res background, 1pi nubar \\
\hline $\begin{array}{l}\text { RvpCC2pi, RvbarnCC2pi, } \\
\text { RvpNC2pi, RvbarnNC2pi }\end{array}$ & Non-res background, 2pi nu \\
\hline $\begin{array}{l}\text { RvnCC2pi, RvbarpCC2pi, } \\
\text { RvnNC2pi, RvbarpNC2pi }\end{array}$ & Non-res background, 2pi nubar \\
\hline BR1gamma, BR1eta & $\begin{array}{l}\text { Resonance decays, radiative decay BR, } \\
\text { single } \text { eta } \mathrm{BR}\end{array}$ \\
\hline Theta_Delta2Npi & Resonance decays, angular distribution \\
\hline $\mathrm{NC}$ & NC cross section scaling \\
\hline AhtBY, BhtBY & DIS form factor, high-twist BY scaling \\
\hline CV1uBY, CV2uBY & DIS form factor, GRV98 PDF correction \\
\hline DISNuclMod & DIS nuclear model modification \\
\hline AGKY_xF1pi, AGKY_pT1pi & DIS AGKY hadronization, pion Feyman $x$, and pion $p_{T}$ \\
\hline FormZone & Hadron formation zone \\
\hline CCQEPauliSupViaKF & Fermi Gas Model, Pauli suppression $k_{F}$ \\
\hline CCQEMomDistroFGtoSF & Fermi Gas Model, CCQE vector form factor \\
\hline MFP_N, FrCEx_N & $\begin{array}{c}\text { Intranuke FSI model, nucleon mfp, } \\
\text { nucleon charge ex }\end{array}$ \\
\hline $\begin{array}{l}\text { FrElas_N, FrInel_N, FrAbs_N, } \\
\text { FrPiProd_N, MFP_pi, FrCEx_pi }\end{array}$ & $\begin{array}{l}\text { Intranuke FSI model, nucleon elastic, } \\
\text { inelastic, absorbtion, nucleon } \pi \text { prod, } \\
\text { pi mfp, pi charge ex }\end{array}$ \\
\hline FrElas_pi, FrInel_pi, & Intranuke FSI model, pi elastic \& inelastic, \\
\hline FrAbs_pi, FrPiProd_pi & pi absorption, pi $\pi$ prod. \\
\hline
\end{tabular}

Table 10.1: GENIE neutrino model parameters considered for cross section systematics. 


\subsection{Detector Systematics}

Systematic uncertainties associated with detector modelling can not be evaluated using the reweighing scheme as they directly affect the features present in the LArTPC image. Detector systematics are assessed by independently varying simulation parameters to their minimum and maximum $1 \sigma$ extreme values and generating $\mathrm{MC}$ samples with the same neutrino interaction vertex. All variations use the same CV calibration. The full reconstruction and analysis chain is used to recompute the final observable under the given variation. Then, the variations are compared on an event by event basis to the CV to determine the change in event rate per energy bin. Due to computing constraints, the contribution of detector systematics are not considered in this thesis. Estimation of the effect of the detector systematics based on preliminary work is discussed here. Three detector variations produce the largest effect on the selected $\nu_{\mu}$ spectrum,

- Modifying the space charge effect model based on a data-driven scaling function. This variation reduces the strength of the space charge effect compared to the $\mathrm{CV}$, and has an impact related to the fiducial volume requirement in both the $1 e 1 \mathrm{P}$ and $1 \mu 1 \mathrm{P}$ selections. The number of selected $1 \mu 1 \mathrm{P}$ events is reduced by $15 \%$ after the variation is applied.

- Scaling the longitudinal diffusion constant by $\pm 1 \sigma$. Longitudinal diffusion is diffusion of ionization electrons in the direction parallel to the TPC electric field and has been measured in MicroBooNE. This effect is expected to smear the charge depositions along the time direction as the initial electron ionization cloud expands as it travels in the drift direction toward the wires. This effect is expected to reduce the number of reconstructed $1 \mu 1 \mathrm{P}$ vertices by approximately $40 \%$.

- In the current simulation, charge induction on neighboring wires is only simulated on the wire closest to the drifting charge. In this variation the model is replaced by a model that includes longer-range charge induction which includes 10 neighboring wires on each side. The main effect can be attributed to reducing the intensity of charge observed on the induction planes and making particles appear with larger 
spread. This effect is expected to reduce the number of reconstructed $1 \mu 1 \mathrm{P}$ vertices by approximately $15 \%$.

\section{$10.5 \nu_{\mu}$ Constraint}

The selected $\nu_{\mu}$ events are used to constrain the systematic uncertainty on the $\nu_{e}$ 's by taking advantage of correlations between $\nu_{\mu}$ and $\nu_{e}$ energy bins. The first component of the constraint relies on the physics mechanism of pion decay which produces the flux of muon and electron flavor neutrinos in the beam. Muon and electron neutrinos below $1 \mathrm{GeV}$ are produced primarily through $\pi^{+}$decay. First, muon neutrinos are produced via $\pi^{+} \rightarrow$ $\mu^{+}+\nu_{\mu}$. Then, antimuon decay produces electron neutrinos through $\mu^{+} \rightarrow e^{+}+\bar{\nu}_{\mu}+\nu_{e}$. Alternatively, the pion can decay directly to a positron and electron neutrino. The second component of the constraint is due to the similarity between the low energy cross sections between $\nu_{\mu}$ and $\nu_{e}$ CCQE scattering, which are strongly correlated. The cross sections are fundamentally similar for muon and electron neutrino scattering because they have a similar Fermi motion, Pauli exclusion, and nuclear effects. The correlated flux and cross sections of muon and electron neutrinos is naturally expressed via the covariance matrix, as the correlation between energy bins are built into the off diagonal elements. The correlation and fractional covariance matrices for the selected $1 e 1 \mathrm{P}$ and $1 \mu 1 \mathrm{P}$ events are shown in Fig. 10.1 for 20 reconstructed energy bins.

The $\mathrm{x}$ and $\mathrm{y}$ axis correspond to a reconstructed energy bin in the $1 \mathrm{e} 1 \mathrm{P}$ and $1 \mu 1 \mathrm{P}$ selection. The $1 e 1 \mathrm{P}$ bins run from 0 to 7 with bin edges $[200,300,400,500,600,700,800,1300]$ $\mathrm{MeV}$ and are associated with the lower left block of the matrix. The $1 \mu 1 \mathrm{P}$ bins run from 7 to 20 with bin edges from $200 \mathrm{MeV}$ to $1500 \mathrm{MeV}$ in increments of $100 \mathrm{MeV}$. In the off diagonal components of the correlation matrix, strong correlations are observed between $\nu_{e}$ and $\nu_{\mu}$ energy bins. Reduced correlation is observed in the last three high energy $\nu_{\mu}$ reconstructed energy bins.

The correlation elements of the covariance matrix provides a constraint when calculating a goodness of fit statistic $\chi^{2}$ for data versus MC when minimized for a model fit. Consider the event differences between an oscillation model fit and the MC prediction for $N$ side-by- 

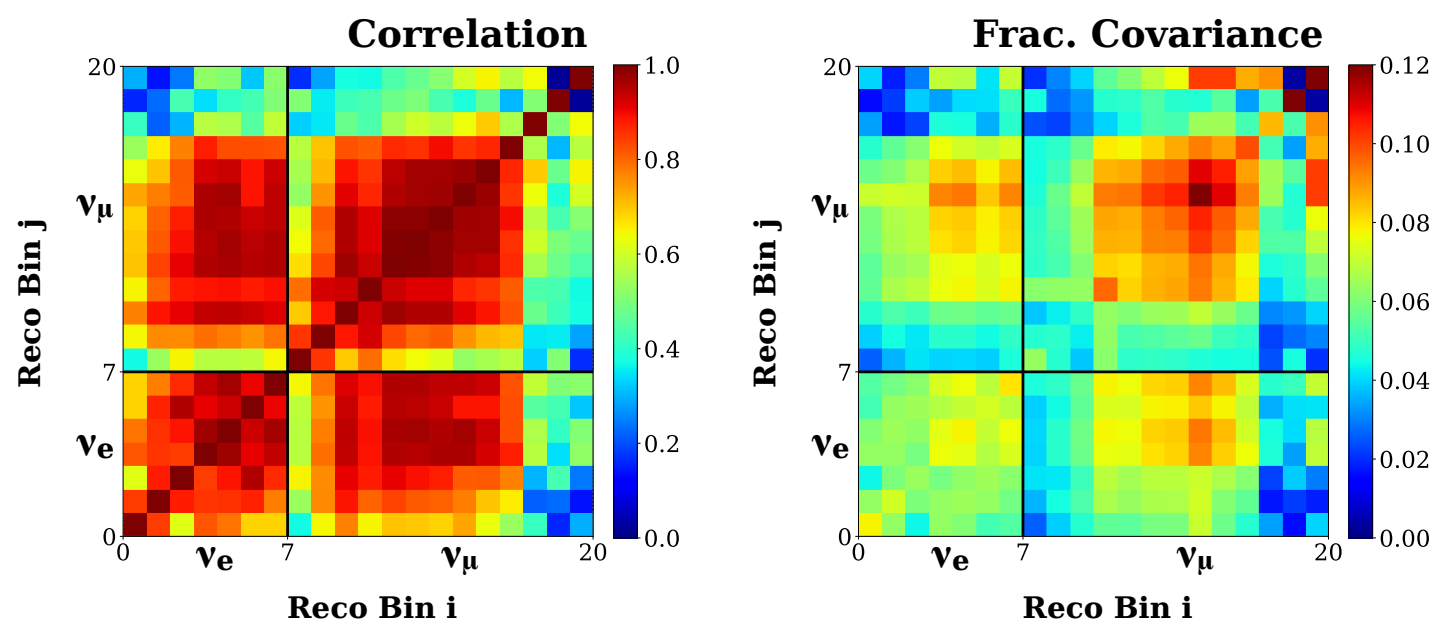

Figure 10.1: Left: Correlation matrix between $1 e 1 \mathrm{P}$ and $1 \mu 1 \mathrm{P}$ reconstructed energy bins for flux and cross section systematic variations. Right: Fractional covariance matrix for the same reconstructed energy binning.

side $\nu_{e}$ and $\nu_{\mu}$ bins,

$$
\Delta_{i}=N_{i}^{\mathrm{fit}}-N_{i}^{\mathrm{MC}}
$$

Then the $\chi^{2}$ between fit and the prediction is then,

$$
\chi^{2}=\sum_{i, j} \Delta_{i} M_{i j}^{-1} \Delta_{j}
$$

where $M_{i j}^{-1}$ is the inverse of the covariance matrix including statistical and systematic uncertainties. Minimizing the $\chi^{2}$ assuming a no oscillation hypothesis, the value of $N_{i}^{\text {fit }}$ and associated uncertainty will be,

$$
N_{i}^{\mathrm{fit}}=N_{i}^{\mathrm{MC}} \pm \sqrt{M_{i i}}
$$

A constraint can now be applied to the $\nu_{\mu}$ fit values, $N_{i}^{\text {fit }}$, such that they are equal to the observed event values within statistical errors, $N_{i}^{\text {data }} \pm \sqrt{N_{i}^{\text {data }}}$, by adding a pull term to the $\chi^{2}$ function. The function then becomes,

$$
\chi^{2}=\sum_{i, j} \Delta_{i} M_{i j}^{-1} \Delta_{j}+\sum_{k} \frac{\left(N_{i}^{\mathrm{fit}}-N_{i}^{\mathrm{data}}\right)^{2}}{N_{k}^{\text {data }}},
$$

Where $i$ and $j$ run over the sum of $\nu_{e}$ and $\nu_{\mu}$ energy bins and $k$ runs over the $\nu_{\mu}$ bins only. 
Taking the derivative and solving the system of equations leads to,

$$
N_{i}^{\mathrm{fit}}=\sum_{k} B_{i k}\left(\sum_{j} C_{k j}^{-1} N_{j}^{\mathrm{MC}}\right),
$$

where,

$$
\begin{gathered}
B_{i j}^{-1}= \begin{cases}M_{i j}^{-1} & \text { for } i, j \leq n_{\nu_{e}} \\
M_{i j}^{-1}+\frac{\delta_{i j}}{N_{i}^{\text {data }}} & \text { for } i, j \geq n_{\nu_{e}}\end{cases} \\
C_{i j}^{-1}= \begin{cases}M_{i j}^{-1} & \text { for } i, j \leq n_{\nu_{e}} \\
M_{i j}^{-1}+\frac{\delta_{i j}}{N_{i}^{\mathrm{MC}}} & \text { for } i, j \geq n_{\nu_{e}}\end{cases}
\end{gathered}
$$

which gives constrained errors on the effective events. The constrained uncertainties on the $\nu_{e}$ bins are obtained by inverting the standard covariance matrix, $M_{i j}^{-1}$, adding $1 / N_{i}^{\text {data }}$ to the diagonal entries of the $\nu_{\mu}$ block, then inverting the resulting matrix. Here we take the number of observed "data" events to be the MC prediction, $N_{i}^{\mathrm{MC}}$. The result of applying the constraint to the seven $\nu_{e}$ reconstructed energy bins is shown in Table 10.2 .

\begin{tabular}{cccccc}
\hline & & \multicolumn{2}{c}{ Systematic-Only Error } & \multicolumn{2}{c}{ Total Error } \\
\cline { 3 - 6 }$\nu_{e}$ bin & Stat-Only Error & Nominal & Constrained & Nominal & Constrained \\
\hline 1 & 0.835 & 0.279 & 0.157 & 0.880 & 0.850 \\
2 & 0.595 & 0.269 & 0.109 & 0.652 & 0.604 \\
3 & 0.496 & 0.254 & 0.086 & 0.557 & 0.503 \\
4 & 0.412 & 0.276 & 0.066 & 0.496 & 0.417 \\
5 & 0.445 & 0.279 & 0.061 & 0.525 & 0.449 \\
6 & 0.499 & 0.270 & 0.073 & 0.567 & 0.504 \\
7 & 0.518 & 0.282 & 0.080 & 0.590 & 0.524 \\
\hline
\end{tabular}

Table 10.2: Fractional uncertainties per $\nu_{e}$ energy bin before and after applying the constraint. Flux and cross section systematic uncertainties are constrained from approximately $25 \%$ to $10 \%$.

The $13.2 \times 10^{20}$ POT event rate predictions after selection are shown in Fig. 10.2 with statistical and systematic uncertainties. Only intrinsic $\nu_{e}$ background is observed after the 
$1 e 1 \mathrm{P}$ selection and is dominated by statistical uncertainties due to the low event rate. $\mathrm{MC}$ statistical uncertainties are not considered as the number of simulated events is in each bin are larger than 10 times the predicted data size.
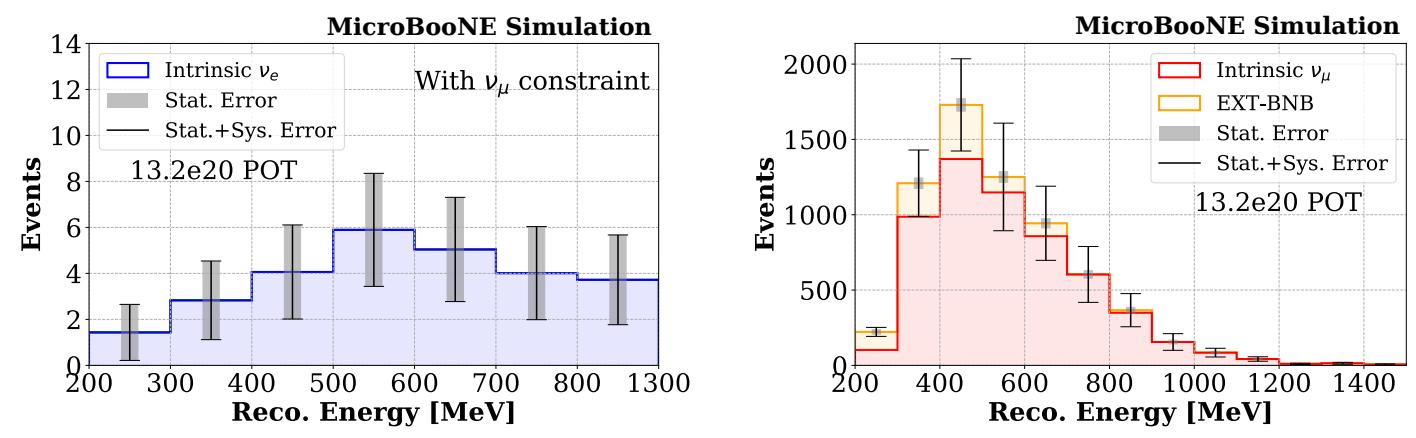

Figure 10.2: Reconstructed energy spectrum scaled to $13.2 \times 10^{20}$ POT for the $1 \mathrm{e} 1 \mathrm{P}$ (left) and $1 \mu 1 \mathrm{P}$ (right) selections including statistical and systematic errors. 


\section{Chapter 11}

\section{Low Energy Excess Search}

With the reconstruction, selection, and systematic uncertainties finalized, simulation is used to estimate the sensitivity for performing a low energy excess search with the a $13.2 \times$ $10^{20}$ POT sample. A statistical analysis is performed to estimate the expected deviation of the signal from the intrinsic $\nu_{e}$ background only hypothesis. This analysis predicts MicroBooNE would observe a $3.8 \sigma$ excess for the MiniBooNE electron-like LEE signal. Next, the sensitivity to high $\Delta m^{2}$ oscillations via $\nu_{e}$ appearance is quantified under a 3 neutrino plus 1 sterile neutrino model given the predicted intrinsic $\nu_{e}$ rate. Finally, the LEE spectrum obtained from CC intrinsic $\nu_{e}$ unfolding is fit to a $3+1$ oscillation model and the best fit point and associated confidence contours are extracted.

\subsection{Significance of an LEE Signal}

After the $1 e 1 \mathrm{P}$ selection is applied only intrinsic $\nu_{e}$ background is predicted at $13.2 \times 10^{20}$ POT using a $1.03 \times 10^{22}$ MC POT equivalent. A simulated low energy excess signal sample is used to estimate the predicted neutrino rate using an $2.8 \times 10^{23} \mathrm{MC}$ POT sample. In this sample, the charge current intrinsic $\nu_{e}$ interactions are enhanced by the unfolded LEE prediction from MiniBooNE as described in Chapter 6. The stacked event rate for the LEE signal and the intrinsic $\nu_{e}$ background is shown in Fig. 11.1.

The LEE signal is concentrated in the less than $500 \mathrm{MeV}$ region and is shown with statistical and constrained systematic uncertainties from flux and cross section contributions. 


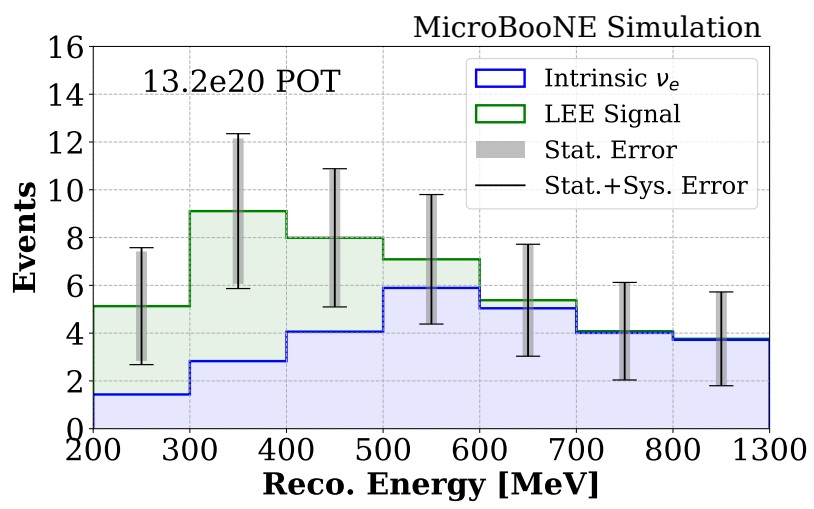

Figure 11.1: Reconstructed energy spectrum for the predicted LEE signal (green) on the intrinsic $\nu_{e}$ only (blue) background for $13.2 \times 10^{20}$ POT. Statistical and systematic uncertainties are shown.

No uncertainties in the unfolding procedure are currently taken into account. To quantify the amount by which the predicted LEE signal deviates from the intrinsic $\nu_{e}$ background a hypothesis test is performed using a $\chi^{2}$ test statistic. Two hypothesis are given for the predicted $13.2 \times 10^{20}$ POT reconstructed neutrino energy event rate. The null hypothesis, $H_{0}$, is the assumption that the spectrum is entirely due to intrinsic $\nu_{e}$ background. The alternative hypothesis, $H_{1}$, is that the rate is due to a combination of intrinsic $\nu_{e}$ background and an electron like LEE signal contribution. A frequentist based approach is used to determine the ability to reject the intrinsic $\nu_{e}$ only hypothesis given a median experiment where the LEE is present. In this procedure both the background and signal plus background distributions are sampled in independent "experiments" given their statistical and correlated systematic uncertainties. Each experiment represents a potential measurement of the reconstructed energy spectrum in MicroBooNE under each hypothesis. The experiments are drawn from the covariance matrix using the Cholosky decomposition Monte Carlo technique. For each hypothesis, H, 10 million experiments are drawn to form a probability density function (PDF), $g$, of $\chi^{2}$ test statistics against the intrinsic background only assumption. The PDF for each hypothesis is,

$$
g\left(\chi^{2} \mid H\right)=\sum_{i}\left(N_{i}^{H}-N_{i}^{\mathrm{bkg}}\right) M_{i j}^{-1}\left(N_{i}^{H}-N_{i}^{\mathrm{bkg}}\right),
$$

where $N^{H}$ is the MC experiment under either the background or background plus signal 
assumption, $N^{\mathrm{bkg}}$ is the intrinsic $\nu_{e}$ only background, and $M_{i j}$ is the covariance matrix of the background. The result of the frequentist test is shown in Fig. 11.2.

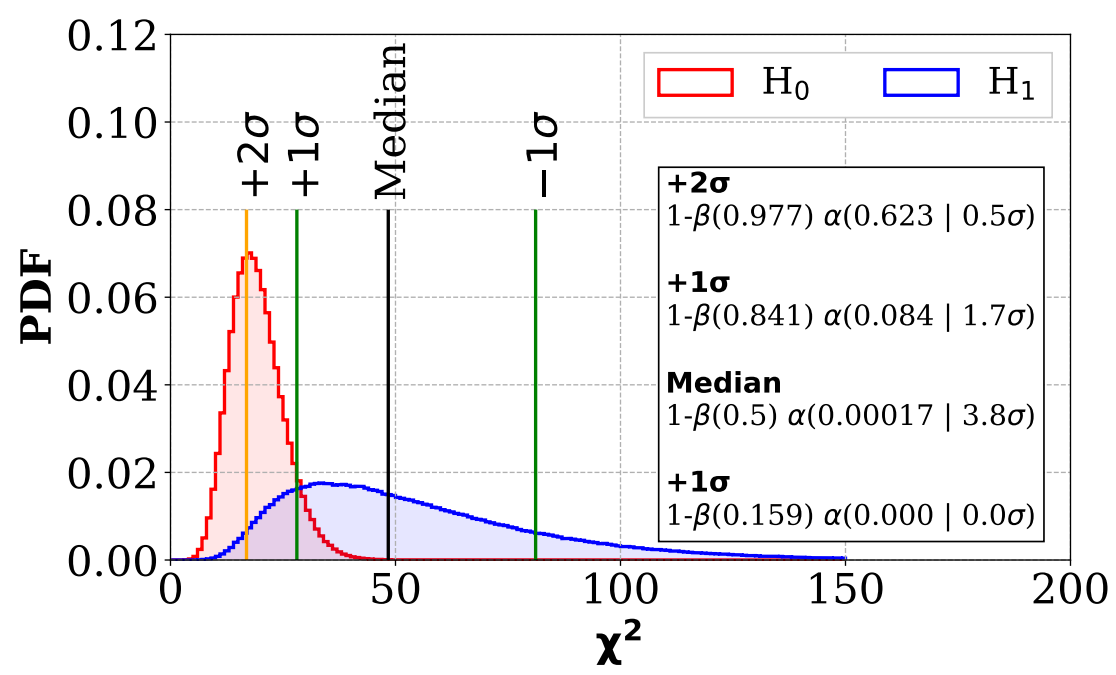

Figure 11.2: Frequentist study for rejecting the intrinsic $\nu_{e}$ only background hypothesis in $13.2 \times 10^{20}$ POT as described in Section 11.1. The red and blue distributions represent the PDF of the $\chi^{2}$ test statistic under the background only and background plus LEE signal respectively.

The red and blue distributions show the $\chi^{2}$ distribution for the background only assumption $\left(H_{0}\right)$ and the background plus LEE signal assumption $\left(H_{1}\right)$ respectively. Each distribution shows the probable amount by which the experiment distribution will deviate from the intrinsic $\nu_{e}$ only background prediction given the associated uncertainties. In the absence of the LEE signal a $\chi^{2} /$ dof value of $25.8 / 20$ is found for comparing background fluctuations with the intrinsic only background. If an LEE signal were to be present, the median experiment would predict a $\chi^{2} /$ dof value of $48 / 20$. Under the intrinsic $\nu_{e}$ background only assumption, this $\chi^{2}$ value has a probability of being found in $0.017 \%$ of experiments which translates to a gaussian $3.8 \sigma$ deviation from the expected mean. A lower bound on the estimated signal significance is estimated using this technique by computing the $+1 \sigma$ and $+2 \sigma$ LEE experiments which represent downward fluctuations of the LEE signal within uncertainties. In $98 \%$ and $84 \%$ of LEE experiments a $\chi^{2}$ value is expected which repre- 
sents at least $0.5 \sigma$ and $1.7 \sigma$ deviations from the background only hypothesis. Due to large statistical uncertainties the distribution of LEE test statistics extends beyond the available $\mathrm{PDF}$ of the background only hypothesis in the $-1 \sigma$ direction. A large MC sample size is required to simulate the extreme tails of the background only distribution.

\subsection{Sensitivity to $3+1$ Oscillations}

The low energy excess prediction can be tested as an oscillation signal given the presence of 1 sterile neutrino with a mass splitting of order $1 \mathrm{eV}^{2}$. The short baseline approximation, which assumes $\Delta m_{\text {solar }}^{2}=\Delta m_{\text {atmospheric }}^{2}=0$, is used to model an additional sterile state neutrino mass splitting as two neutrino mixing. In the case of MicroBooNE, with $L / E \sim$ $1 \mathrm{~m} / \mathrm{MeV}$, the sensitivities to the solar and atmospheric mass splittings are small. The sensitivity to $3+1$ oscillations is quantified via the $\nu_{e}$ appearance channel where intrinsic $\nu_{\mu}$ oscillate to additional $\nu_{e}$. In this theory the $\nu_{e}$ appearance probability for $\nu_{\mu} \rightarrow \nu_{e}$ transitions is,

$$
P_{\nu_{\mu} \rightarrow \nu_{e}}=4\left|U_{e 4}\right|^{2}\left|U_{\mu 4}\right|^{2} \sin ^{2}\left(1.27 \Delta m_{41}^{2} L / E\right)
$$

where $4\left|U_{e 4}\right|^{2}\left|U_{\mu 4}\right|^{2}=\sin ^{2} 2 \theta_{e \mu}$.

Given the predicted intrinsic $\nu_{e}$ background rate the sensitivity to sterile neutrino oscillations is performed using the raster scan method. In this procedure, the $\Delta m_{41}^{2}$ vs. $\sin ^{2} 2 \theta_{e \mu}$ space is discretized into a grid and an oscillation spectrum is predicted by Equation 11.2 for a given pair of oscillation parameters applied to a so called "full osc" sample. The full osc sample is a fully transmuted sample of intrinsic $\nu_{\mu}$ events which have been fully oscillated to $\nu_{e}$ in the simulation. The neutrinos are produced with the $\nu_{\mu}$ flux and interact as $\nu_{e}$ in the detector via the $\nu_{e}$ cross section. The full osc sample is passed through the complete reconstruction and $1 e 1 \mathrm{P}$ analysis chain. For a given true baseline, $L$, and true neutrino energy, $E$, the sample is reweighed at each $\left(\Delta m_{41}^{2}, \sin ^{2} 2 \theta_{e \mu}\right)$ point to give a predicted oscillation spectrum on top of the intrinsic $\nu_{e}$ background. The oscillated spectrum is compared with the intrinsic $\nu_{e}$ background at each point using a $\chi^{2}$ defined as,

$$
\chi^{2}=\sum_{i}\left(N_{i}^{\mathrm{osc}}-N_{i}^{\mathrm{bkg}}\right) M_{i j}^{-1}\left(N_{i}^{\mathrm{osc}}-N_{i}^{\mathrm{bkg}}\right),
$$


where $N_{i}^{\mathrm{osc}}$ and $N_{i}^{\mathrm{bkg}}$ are the oscillated and no-oscillations background spectrum respectively. The correlation matrix including the full osc sample is shown in Fig. 11.3.

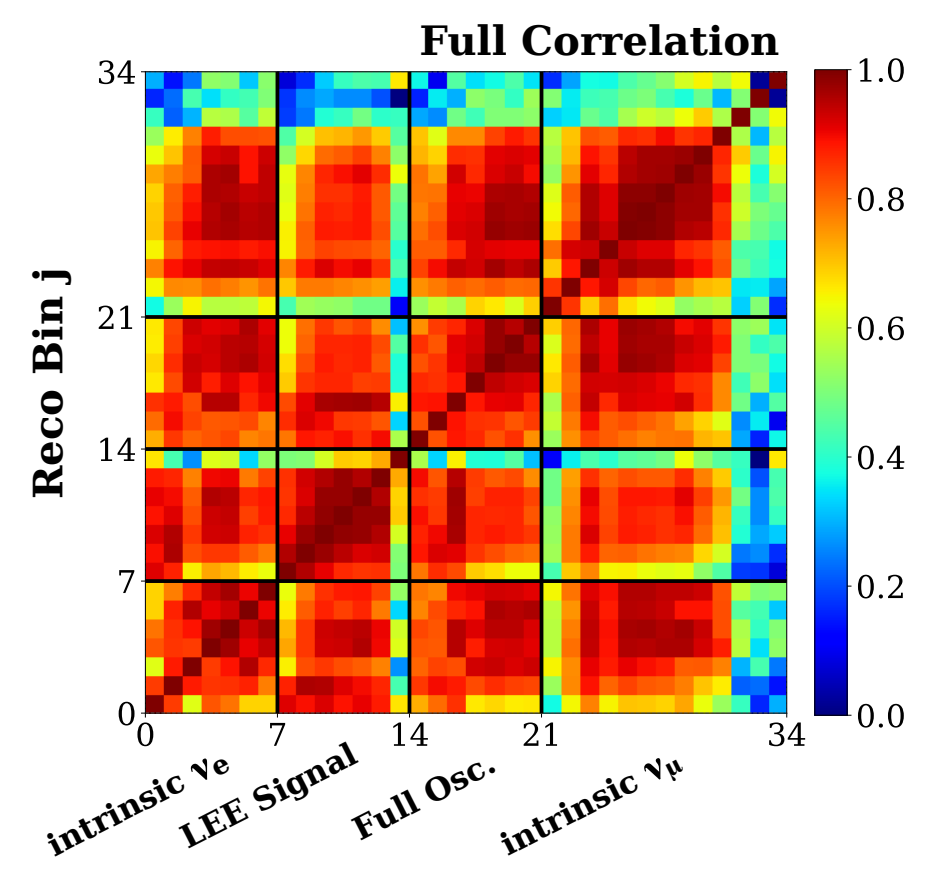

Reco Bin i

Figure 11.3: Correlation matrix between four samples: intrinsic $\nu_{e}$, the LEE signal, the full osc sample, and the intrinsic $\nu_{\mu}$ background. Strong correlations are observed between energy bins in each sample.

The results of the raster scan are shown in Fig. 11.4. The 90\% confidence level exclusion curve is shown as the red line.

For points to the right of the red curve, the oscillated spectrum gives a $\chi^{2}$ value that is large enough to be resolved given the intrinsic $\nu_{e}$ background rate within uncertainties. For grid points to the left of the curve the oscillated spectrum cannot be resolved given the intrinsic $\nu_{e}$ spectrum with uncertainty. In this region the amplitude and shape of the oscillations are comparable to the intrinsic $\nu_{e}$ uncertainty. The raster scan line well covers the LSND $99 \%$ allowed region shown in solid green for $\sin ^{2} 2 \theta_{\mu e} \sim 10^{-3}$ and $\Delta m^{2} \sim 1 \mathrm{eV}^{2}$ oscillations. A global fit to neutrino oscillation data [47] is shown as the $3 \sigma$ allowed region in blue. 


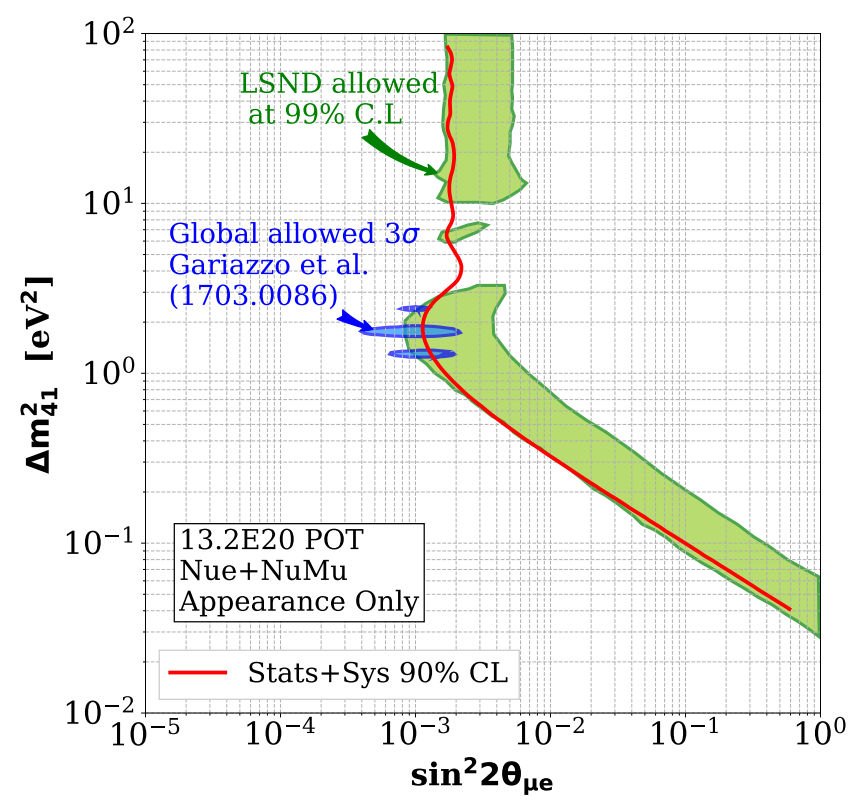

Figure 11.4: One sided raster scan to test for sensitivity to $\Delta m_{41}^{2}$ oscillations as described in the text.

The low energy excess signal prediction is fit to a $3+1$ oscillation model using the combined fit procedure developed by the MiniBooNE collaboration and implemented by the author in SBNfit [48. For a given data set the reconstructed neutrino energy distribution for $\nu_{e}$ and $\nu_{\mu}$ candidates are compared to their corresponding Monte Carlo distributions for various signals across the oscillation space to find the set of parameters which minimizes Equation 11.3. An iterative approach is taken such that the covariance matrix is first calculated assuming there is no signal and is kept constant while varying the oscillation parameters. Once the minimum has been found using the covariance matrix with no signal, the matrix is recalculated with the oscillation spectrum at the best fit point. A new minimization is then performed while keeping the updated covariance matrix fixed throughout. This process is iterated until a convergence criterion is met. The result is a set of best fit parameters that correspond to the minimum $\chi^{2}$ surface when the covariance matrix is fixed at the best fit parameters themselves. The result of the oscillations fit to the LEE spectrum is shown as the red star in Fig. 11.5 where the fit converged in 3 iterations to the 
best fit point of $\left(\Delta m_{41}^{2}, \sin ^{2} 2 \theta_{e \mu}\right)=(0.063,0.794)$ with a $\chi^{2}$ of 5.06 assuming two degrees of freedom.

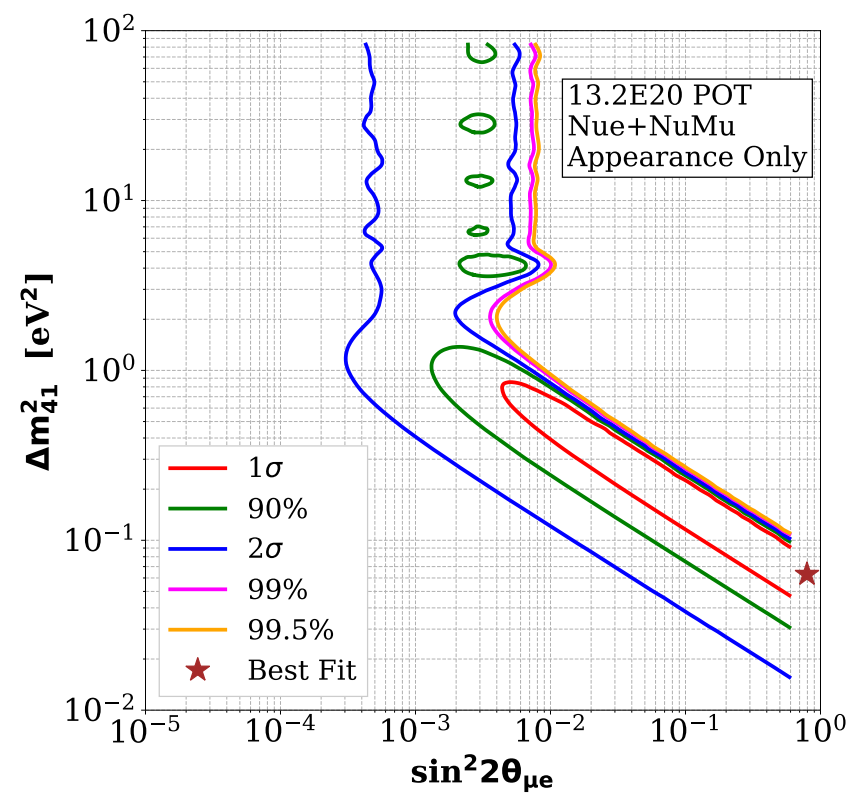

Figure 11.5: Oscillations fit for $\nu_{e}$ appearance applied to the LEE signal as described in the text. The best fit point is shown as the red star. Various confidence levels are shown using the $\Delta \chi^{2}$ prescription. The allowed regions can be compared to the MiniBooNE result in Fig. 5.5

The allowed contours at the best fit point are calculated using the $\Delta \chi^{2}$ prescription. Points enclosed at each region represent the set of oscillation parameters which are within the given confidence, or $\Delta \chi^{2}$, of the best fit point given two degrees of freedom throughout the space. Frequentist studies following the Feldman-Cousins approach [49] have been shown that the $\Delta \chi^{2}$ distribution across the oscillation parameter space varies from 1 degree of freedom to 5.5 degree of freedom. No significant change in the best fit point location nor the structure of the confidence regions change when the degrees of freedom per grid point is determined. The $90 \%$ allowed confidence region represents a $\Delta \chi^{2}=4.61$ from the best fit point and is effected by oscillation shape as closed islands are observed at high $\Delta m_{41}^{2}$. For confidence regions of $99 \%$ and above, no closed contours are observed. The oscillation 
spectrum at the best fit point is shown in Fig. 11.6.

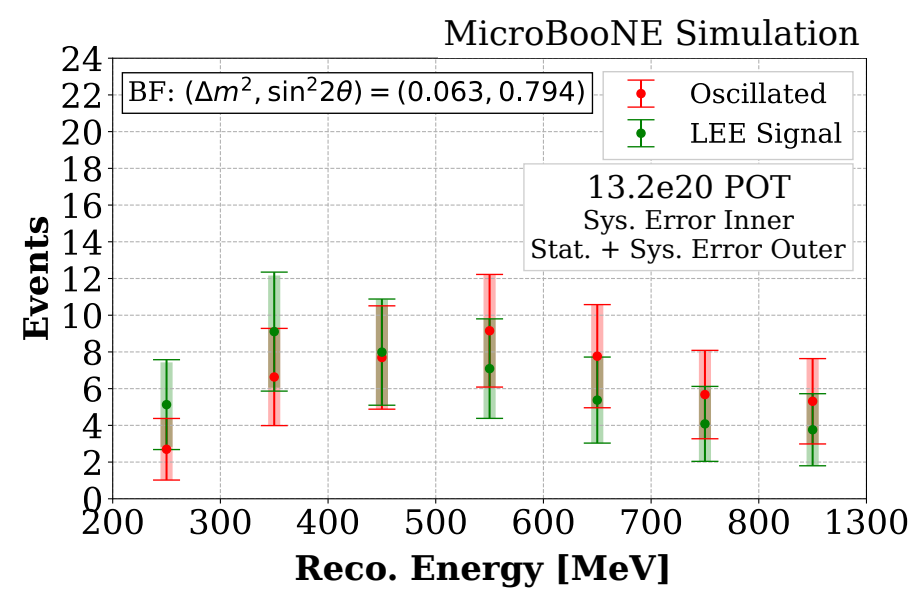

Figure 11.6: The intrinsic $\nu_{e}$ background and low energy excess reconstructed energy prediction (green) compared to the best fit $\nu_{\mu} \rightarrow \nu_{e}$ oscillation spectrum (red).

The amplitude of oscillations at the best fit point are commensurate with the LEE signal and intrinsic $\nu_{e}$ background, but some shape difference is observed. Better resolution of the oscillation signal can be achieved with reduced statistical uncertainties. 


\section{Chapter 12}

\section{Conclusions}

This thesis presented a first of its kind reconstruction and analysis chain for identifying CCQE-like muon and electron neutrinos in the MicroBooNE detector using image based reconstruction techniques. A hybrid of hand designed and deep learning computer vision algorithms were developed and applied to MicroBooNE data and simulation to estimate CCQE efficiencies and backgrounds.

The MiniBooNE low energy $12.84 \times 10^{20}$ POT CCQE data was unfolded to provide a data driven prediction of the low energy excess in MicroBooNE for the first time. Under a model of the low energy excess which is due to an enhancement of the $\nu_{e}$ charge current rate, the excess is found to be concentrated below $800 \mathrm{MeV}$ in true neutrino energy with appreciable energy dependence. For the full MicroBooNE $13.2 \times 10^{20}$ POT dataset, 654 low energy excess events are predicted of which 192 events are expected to be topologically one electron and one proton.

An image based reconstruction software was presented along with efficiencies at each step for identifying CCQE topologies in large multi-wire LArTPC images. Algorithmic techniques for $2 \mathrm{D}$ and $3 \mathrm{D}$ cosmic ray tracking and rejection within the event image reduced the image complexity for neutrino search. Along with the in-time PMT flash, the detector region where neutrino activity likely occurred was isolated to establish a region of interest. A new deep learning algorithm was applied inside the region of interest for a per pixel classification to address the challenging task of track and shower classification. Next, the $3 \mathrm{D}$ vertex of neutrino scattering for muon and electron type neutrinos provided an anchor 
for pattern recognition of the final state particles. Muon neutrino interactions were reconstructed using a tracking algorithm based on stochastic search to identify the presence of muon and proton particles. Electron neutrinos were reconstructed by extracting detailed features of the outgoing electron's ionization pattern.

A low energy CCQE-like neutrino selection was presented using likelihood and decision tree based algorithms and compared to the Run 1 MicroBooNE dataset. The muon selection combined 3D track features into two likelihoods for an integrated selection efficiency of $18 \%$ with cosmic and neutrino background rejection capability of $99.97 \%$ and $98.7 \%$ respectively. After selection, good agreement is observed between data and Monte Carlo features and provides sufficient statistics for constraining the $\nu_{e}$ systematic uncertainties. The electron selection featured a ensemble based machine learning approach combining support vector machine and gradient boosted decision tree classifiers. Two support vector machine classifiers were developed based on a multi-particle identification convolutional neural network and the 3D track features of proton and electron kinematics. A boosted decision tree classifier was developed by identifying strong topological features of the outgoing electron shower combined with a $d E / d x$ estimation of the proton yielding a global $1 e 1 \mathrm{P}$ efficiency of $15 \%$. Two $1 e 1 \mathrm{P}$ candidate neutrinos were identified via handscan in the $5 \times 10^{19}$ POT data set given a $2.51 \pm 0.25$ event expectation. Combined, the classifiers achieved an intrinsic $\nu_{e}$ only background for a low energy excess search with an intergrated efficiency of $\sim 5 \%$.

The $\nu_{\mu}$ and $\nu_{e}$ systematic uncertainties related to GENIE cross section and BNB flux were characterized. Given the measured $\nu_{\mu}$ spectrum at $13.2 \times 10^{20} \mathrm{POT}$, the $\nu_{e}$ systematic uncertainties are constrained from approximately $25 \%$ to below $10 \%$. The statistical uncertainties predicted $\nu_{e}$ event rate are shown to dominate.

The significance of a MiniBooNE low energy excess signal is profiled by performing a frequentist study of rejecting the intrinsic $\nu_{e}$ only background (null) hypothesis. The median experiment given the presence of an electron-like MiniBooNE low energy excess signal would indicate a $3.8 \sigma$ deviation from the null hypothesis given statistical and systematic uncertainties. The intrinsic $\nu_{e}$ only background and LEE signal prediction binned in reconstructed neutrino energy (see Fig. 11.1) is shown in Table 12.1.

In 2019 an updated MicroBooNE simulation will come online with the promise of im- 


\begin{tabular}{|l|c|c|c|c|c|c|c|}
\hline Reco. Energy Bin $[\mathrm{MeV}]$ & $\mathbf{2 0 0}$ & $\mathbf{3 0 0}$ & $\mathbf{4 0 0}$ & $\mathbf{5 0 0}$ & $\mathbf{6 0 0}$ & $\mathbf{7 0 0}$ & $\mathbf{8 0 0 - 1 3 0 0}$ \\
\hline Intrinsic $\nu_{\mathbf{e}}$ Background & 1.43 & 2.82 & 4.06 & 5.89 & 5.04 & 4.01 & 3.72 \\
\hline Electron-like LEE Signal & 3.69 & 6.27 & 3.92 & 1.19 & 0.33 & 0.06 & 0.04 \\
\hline
\end{tabular}

Table 12.1: Reconstructed neutrino energy bin-by-bin event rate expectation for the intrinsic $\nu_{e}$ background and electron-like LEE signal for $13.2 \times 10^{20}$ POT corresponding to Fig. 11.1.

proved signal processing, noise removal, and with advanced detector effects simulation such as the dynamic induced charge effect. The updated simulation with respect to the signal processing is expected to have a major impact on the image quality of the two induction planes. The current $1 e 1 \mathrm{P}$ analysis relies primarily on the collection plane for pattern recognition. With this requirement relaxed in updated simulation, an expected gain in $1 e 1 \mathrm{P}$ efficiency is expected while keeping the backgrounds low. Assuming the same $13.2 \times 10^{20}$ POT dataset, the expected low energy excess significane with intrinsic $\nu_{e}$ only backgrounds is shown in Table 12.2. An increase in ability to reject the intrinsic $\nu_{e}$ background hypothesis increases with improved efficiency. Additionally a liquid argon based near detector, called the Short Baseline Near Detector (SBND), will come online in 2020/2021 and will take neutrino data in the same beam-line [50] as MicroBooNE which will help reduce systematic uncertainties for a LEE or oscillations search.

\begin{tabular}{l|l|l} 
Efficiency & Median LEE Sig. & $+\mathbf{1} \sigma$ Sig. \\
\hline $5 \%$ (nominal) & $3.8 \sigma$ & $1.7 \sigma$ \\
\hline $7.5 \%$ & $4.5 \sigma$ & $2.2 \sigma$ \\
\hline $10 \%$ & $5.1 \sigma$ & $2.6 \sigma$
\end{tabular}

Table 12.2: Low energy excess signal significance for frequentist studies with statistical and systematic errors included. The nominal significance of $3.8 \sigma$ is shown at $5 \% 1 \mathrm{e} 1 \mathrm{P}$ efficiency as described in this thesis. An efficiency improvement of $50 \%$ and $100 \%$ over the nominal result would improve the median LEE significance. The right most column shows the $1 \sigma$ downward fluctuation of the LEE significance.

Finally, the sensitivity to $3+1$ sterile oscillations was also presented by performing a raster scan in the high $\Delta m^{2}$ and $\sin ^{2} 2 \theta_{e \mu}$ space. The majority of the LSND allowed region 
is covered given muon to electron neutrino flavor transitions. The simulated electron-like LEE signal (shown in Fig. 11.1) is fit to $3+1$ oscillations and found to have a best fit point of $\left(\Delta m_{41}^{2}, \sin ^{2} 2 \theta_{e \mu}\right)=(0.063,0.794)$. The $90 \%$ confidence allowed region is largely consistent with interpreting the MicroBooNE LEE as $>0.1 \mathrm{eV}^{2}$ oscillations.

This thesis explored MicroBooNE's sensitivity to an electron-like low energy excess signal using a custom end-to-end reconstruction and analysis chain. Other groups in the MicroBooNE collaboration are exploring the photon-like nature of the LEE interpreted as an excess of neutral current $\Delta$ production followed by $\Delta$ radiative decay on argon [51] using the Pandora-based reconstruction approach. Statistical analysis shows that MicroBooNE will have an average of $1.87 \sigma$ excess for a photon-like MiniBooNE LEE signal with $6.6 \times 10^{20}$ POT. Both the electron and photon based searches in MicroBooNE have plans for processing the full $13.2 \times 10^{20}$ POT dataset to determine the nature of the MiniBooNE LEE. 


\section{Bibliography}

[1] The MicroBooNE Collaboration. Updated Neutrino Beam Timing Plots. https://microboone-docdb.fnal.gov/cgi-bin/private/ShowDocument?docid=5390, 2017.

[2] The MicroBooNE Collaboration. Booster Neutrino Flux Prediction at MicroBooNE. https://microboone.fnal.gov/wp-content/uploads/MICROBOONE-NOTE1031-PUB.pdf, 2018.

[3] LSND Collaboration. Evidence for neutrino oscillations from the observation of $\bar{\nu}_{e}$ appearance in a $\bar{\nu}_{\mu}$ beam. Phys. Rev. D, 64:112007, Nov 2001.

[4] MiniBooNE Collaboration. Significant Excess of Electronlike Events in the MiniBooNE Short-Baseline Neutrino Experiment. Phys. Rev. Lett., 121:221801, Nov 2018.

[5] X.-G. Lu, L. Pickering, S. Dolan, G. Barr, D. Coplowe, Y. Uchida, D. Wark, M. O. Wascko, A. Weber, and T. Yuan. Measurement of nuclear effects in neutrino interactions with minimal dependence on neutrino energy. Phys. Rev. C, 94:015503, Jul 2016.

[6] M. et al. Tanabashi. Review of particle physics. Phys. Rev. D, 98:030001, Aug 2018.

[7] The MicroBooNE Collaboration. Convolutional neural networks applied to neutrino events in a liquid argon time projection chamber. Journal of Instrumentation, 12(03):P03011, 2017.

[8] The MicroBooNE Collaboration. A Deep Neural Network for Pixel-Level Electro- 
magnetic Particle Identification in the MicroBooNE Liquid Argon Time Projection Chamber, 2018.

[9] The MicroBooNE Collaboration. The Pandora multi-algorithm approach to automated pattern recognition of cosmic-ray muon and neutrino events in the MicroBooNE detector. The European Physical Journal C, 78(1):82, Jan 2018.

[10] The MicroBooNE Collaboration. Ionization electron signal processing in single phase LArTPCs. part II. data/simulation comparison and performance in MicroBooNE. Journal of Instrumentation, 13(07):P07007-P07007, jul 2018.

[11] The MicroBooNE Collaboration. Noise characterization and filtering in the MicroBooNE liquid argon TPC. Journal of Instrumentation, 12(08):P08003-P08003, aug 2017.

[12] J. M. Conrad and M. H. Shaevitz. Sterile neutrinos: An introduction to experiments, 2016.

[13] Pietro Antonioli et al. New Journal of Physics, 6:114-114, sep 2004.

[14] The MiniBooNE Collaboration. Neutrino flux prediction at MiniBooNE. Phys. Rev. D, 79:072002, Apr 2009.

[15] The HARP Collaboration. Measurement of the production cross-section of positive pions in the collision of $8.9 \mathrm{GeV} / \mathrm{c}$ protons on beryllium. The European Physical Journal C, 52(1):29-53, Sep 2007.

[16] The SciBooNE Collaboration. Measurement of $K^{+}$production cross section by $8 \mathrm{GeV}$ protons using high-energy neutrino interactions in the SciBooNE detector. Phys. Rev. D, 84:012009, Jul 2011.

[17] Unexplained Excess of Electronlike Events from a 1-GeV Neutrino Beam. Phys. Rev. Lett., 102:101802, Mar 2009.

[18] The MiniBooNE Collaboration. The MiniBooNE detector. Nuclear Instruments and Methods in Physics Research Section A: Accelerators, Spectrometers, Detectors and Associated Equipment, 599(1):28 - 46, 2009. 
[19] ArgoNeuT Collaboration. First observation of low energy electron neutrinos in a liquid argon time projection chamber. Phys. Rev. D, 95:072005, Apr 2017.

[20] G. D'Agostini. A multidimensional unfolding method based on bayes' theorem. Nuclear Instruments and Methods in Physics Research Section A: Accelerators, Spectrometers, Detectors and Associated Equipment, 362(2):487 - 498, 1995.

[21] Andreas Höcker and Vakhtang Kartvelishvili. Svd approach to data unfolding. Nuclear Instruments and Methods in Physics Research Section A: Accelerators, Spectrometers, Detectors and Associated Equipment, 372(3):469 - 481, 1996.

[22] J. A. Formaggio and G. P. Zeller. From ev to eev: Neutrino cross sections across energy scales. Rev. Mod. Phys., 84:1307-1341, Sep 2012.

[23] Teppei Katori. Meson exchange current (MEC) models in neutrino interaction generators. AIP Conference Proceedings, 1663(1):030001, 2015.

[24] C. Andreopoulos et al. The GENIE neutrino Monte Carlo generator. Nuclear Instruments and Methods in Physics Research Section A: Accelerators, Spectrometers, Detectors and Associated Equipment, 614(1):87 - 104, 2010.

[25] Yichen Li et al. Measurement of longitudinal electron diffusion in liquid argon. Nuclear Instruments and Methods in Physics Research Section A: Accelerators, Spectrometers, Detectors and Associated Equipment, 816:160 - 170, 2016.

[26] R. Brun and F. Rademakers. ROOT: An object oriented data analysis framework. Nucl. Instrum. Meth., A389:81-86, 1997.

[27] V. Genty, K. Terao, and T. Wongjirad. LArCV: LArTPC Image Processing/Analysis Framework for Deep Learning.

[28] LArOpenCV: Liquid Argon Open Source Computer Vision.

[29] G. Bradski. The OpenCV Library. Dr. Dobb's Journal of Software Tools, 2000.

[30] The MicroBooNE Collaboration. Design and construction of the MicroBooNE detector. Journal of Instrumentation, 12(02):P02017-P02017, feb 2017. 
[31] D. Heck, J. Knapp, J. N. Capdevielle, G. Schatz, and T. Thouw. CORSIKA: A Monte Carlo code to simulate extensive air showers. 1998.

[32] The MicroBooNE Collaboration". Study of Space Charge Effects in MicroBooNE. 2016.

[33] P. E. Hart, N. J. Nilsson, and B. Raphael. A formal basis for the heuristic determination of minimum cost paths. IEEE Transactions on Systems Science and Cybernetics, 4(2):100-107, July 1968.

[34] Olaf Ronneberger, Philipp Fischer, and Thomas Brox. U-Net: Convolutional Networks for Biomedical Image Segmentation. In Nassir Navab, Joachim Hornegger, William M. Wells, and Alejandro F. Frangi, editors, Medical Image Computing and ComputerAssisted Intervention - MICCAI 2015, pages 234-241, Cham, 2015. Springer International Publishing.

[35] Kaiming He, Xiangyu Zhang, Shaoqing Ren, and Jian Sun. Deep Residual Learning for Image Recognition. CoRR, abs/1512.03385, 2015.

[36] Yangqing Jia, Evan Shelhamer, Jeff Donahue, Sergey Karayev, Jonathan Long, Ross Girshick, Sergio Guadarrama, and Trevor Darrell. Caffe: Convolutional Architecture for Fast Feature Embedding. In Proceedings of the 22Nd ACM International Conference on Multimedia, MM '14, pages 675-678, New York, NY, USA, 2014. ACM.

[37] LArbys fork of Caffe.

[38] T. Tieleman and G. Hinton. Lecture 6.5-RmsProp: Divide the gradient by a running average of its recent magnitude. COURSERA: Neural Networks for Machine Learning, 2012.

[39] Martin J. Berger, J S. Coursey, and M A. Zucker. ESTAR, PSTAR, and ASTAR: Computer Programs for Calculating Stopping-Power and Range Tables for Electrons, Protons, and Helium Ions. 1999.

[40] Artur M. Ankowski and Jan T. Sobczyk. Argon spectral function and neutrino interactions. Phys. Rev. C, 74:054316, Nov 2006. 
[41] The ArgoNeuT Collaboration. A study of electron recombination using highly ionizing particles in the ArgoNeuT liquid argon TPC. Journal of Instrumentation, 8(08):P08005-P08005, aug 2013.

[42] Martín Abadi et al. TensorFlow: Large-scale machine learning on heterogeneous systems, 2015. Software available from tensorflow.org.

[43] Tianqi Chen and Carlos Guestrin. XGBoost: A Scalable Tree Boosting System. In Proceedings of the 22Nd ACM SIGKDD International Conference on Knowledge Discovery and Data Mining, KDD '16, pages 785-794, New York, NY, USA, 2016. ACM.

[44] Byron P. Roe. Statistical errors in Monte Carlo estimates of systematic errors. Nuclear Instruments and Methods in Physics Research Section A: Accelerators, Spectrometers, Detectors and Associated Equipment, 570(1):159 - 164, 2007.

[45] C. Mariani, G. Cheng, J. M. Conrad, and M. H. Shaevitz. Improved parametrization of $K^{+}$production in $p$-be collisions at low energy using feynman scaling. Phys. Rev. D, 84:114021, Dec 2011.

[46] MiniBooNE Collaboration. Improved search for $\bar{\nu}_{\mu} \rightarrow \bar{\nu}_{e}$ oscillations in the miniboone experiment. Phys. Rev. Lett., 110:161801, Apr 2013.

[47] S. Gariazzo, C. Giunti, M. Laveder, and Y. F. Li. Updated global 3+1 analysis of short-baseline neutrino oscillations. Journal of High Energy Physics, 2017(6):135, Jun 2017.

[48] D. Cianci, A. Furmanski, G. Karagiorgi, and M. Ross-Lonergan. Prospects of light sterile neutrino oscillation and $c p$ violation searches at the fermilab short baseline neutrino facility. Phys. Rev. D, 96:055001, Sep 2017.

[49] Gary J. Feldman and Robert D. Cousins. Unified approach to the classical statistical analysis of small signals. Phys. Rev. D, 57:3873-3889, Apr 1998.

[50] The MicroBooNE Collaboration. A Proposal for a Three Detector Short-Baseline Neutrino Oscillation Program in the Fermilab Booster Neutrino Beam, 2015. 
[51] The MicroBooNE Collaboration. The MicroBooNE Search for Single Photon Events (Public Note 1041). 2018.

[52] The MicroBooNE Collaboration. Michel electron reconstruction using cosmic-ray data from the MicroBooNE LArTPC. Journal of Instrumentation, 12(09):P09014-P09014, sep 2017. 


\section{Part I}

Appendices 


\section{Appendix A}

\section{Department of Energy Office of Science Highlight}

The SSNet paper was featured on the Department of Energy Office of Science Highlights webpage in January 2019 and is reproduced below. The paper has been accepted for publication in the Physical Review D with preprint available at [8]. 


\section{High Energy Physics (HEP)}

01.30 .19

\section{MicroBooNE, Machine Learning, and Liquid Argon}

\section{The MicroBooNE experiment demonstrates the use of machine learning to interpret images made by a liquid-argon particle detector.} The Science

Decades ago, scientists studying tracks created by subatomic particles as they traveled through a detector identified the types of particles manually. The use of machine learning to interpret the image data is a technological leap forward. Now, the MicroBooNE neutrino experiment developed a type of machine learning algorithm—a convolutional neural network. The algorithm converts data from the tracks into information about the particles that generated them.

\section{The Impact}

The MicroBooNE team showed that its new machinelearning algorithm performs exceedingly well. It interprets simulated data and real data similarly. This algorithm is a valuable development for current and future neutrino experiments that use liquid-argon detector technology. It will help scientists learn about the properties of the neutrino and the fundamental laws of

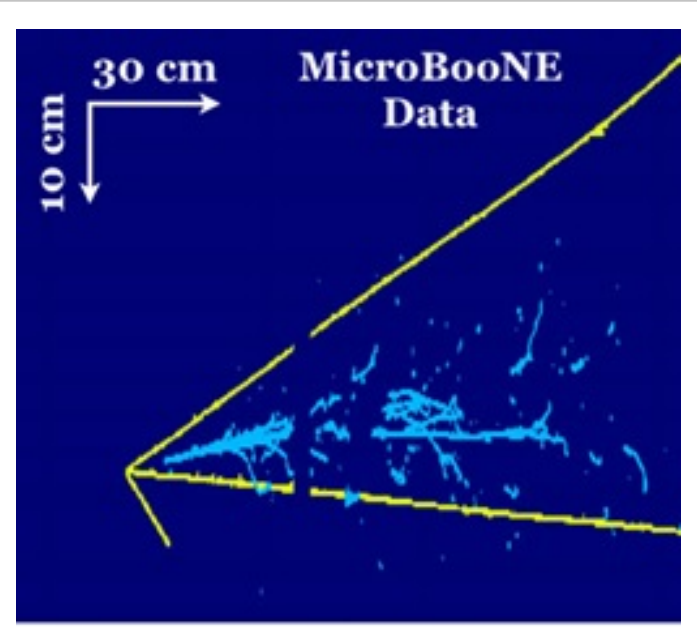

Image courtesy of Fermilab

This event display shows the result of a muon neutrino candidate interacting inside the MicroBooNE detector at Fermilab. The cyan shows the energy deposited in electromagnetic shower-like topologies. The yellow shows energy deposited in other line-like topologies. nature.

\section{Summary}

Researchers on the MicroBooNE neutrino experiment at Fermilab designed a type of machine learning algorithm, convolutional neural networks, to identify subatomic particles that interact in the MicroBooNE detector. The neural network sorted individual pixels coming from images made by a liquid-argon time projection chamber. The neural network, called U-ResNet, distinguished between two types of pixels: those that were a part of a track-like particle trajectory and those that were a part of a shower-like particle trajectory.

Track-like trajectories, made by particles such as a muon or proton, consist of a line with small curvature. Shower-like trajectories, produced by particles such as an electron or photon, are more complex topological features with many branching trajectories. This distinction is important because separating these types of topologies can be difficult for traditional algorithms. Not only that, shower-like shapes are produced when electrons and photons interact in the detector, and these two particles are often an important signal or 
background in physics analyses.

The team demonstrated that U-ResNet not only performs well, but also works in a similar fashion when presented with simulated data and real data. The latter is the first time this has been demonstrated for data from a liquid-argon neutrino detector. Convolutional neural networks are valuable to current and future neutrino experiments that will use liquid-argon neutrino detectors. The more demonstrations there are that these algorithms work on real detector data, the more confidence the community can have that convolutional neural networks will help elucidate the properties of the neutrino and the fundamental laws of nature.

\section{Contact}

Victor Genty

Columbia University

vg2337@columbia.edu

Kazuhiro Terao

SLAC National Accelerator Laboratory

kterao@slac.stanford.edu

Taritree Wongjirad

Tufts University

Taritree.Wongjirad@tufts.edu

\section{Funding}

This work was supported by the Department of Energy, Office of Science, Offices of High Energy Physics and Nuclear Physics; National Science Foundation; Swiss National Science Foundation; Science and Technology Facilities Council of the United Kingdom; and The Royal Society (United Kingdom). Additional support for the laser calibration system and cosmic ray tagger was provided by the Albert Einstein Center for Fundamental Physics.

\section{Publications}

C. Adams, M. Alrashed, R. An, et al. (MicroBooNE Collaboration), "A deep neural network for pixel-level electromagnetic particle identification in the MicroBooNE liquid argon time projection chamber." Fermilab, FERMILAB-PUB-18-231-ND, Batavia, Illinois (2018). [arXiv: 1808.07269]

\section{Related Links}

Fermilab press release: MicroBooNE demonstrates use of convolutional neural networks on liquid-argon TPC data for first time ${ }^{\pi}$

\section{Highlight Categories}

Program: HEP, NP

Performer/Facility: University, DOE Laboratory, SC User Facilities, HEP User Facilities, FermilabAC

Additional: Collaborations, Non-DOE Interagency Collaboration, International Collaboration

Last modified: 1/30/2019 10:43:39 AM

http://science.energy.gov/hep/highlights/2019/hep-2019-01-c?p=1 


\section{Appendix B}

\section{Michel Electron Reconstruction}

\section{B.1 Michel Electron Sideband}

\section{B.1.1 Motivation}

To validate the pixel to $\mathrm{MeV}$ calibration described in Section 8.8.5 the energy distribution from Michel electron decays of cosmic ray muons is reconstructed. Along with the $\pi^{0}$ invariant mass distribution, the Michel distribution will serve as one of two calibration factors to calibrate the energy scale and resolution for electron showers across the MiniBooNE electron like low energy region. The Michel distribution provides a validation of the of the electron energy in the lowest true neutrino energy bin for $1 e 1 \mathrm{P}$ candidates. Additionally, the Michel sample helps build confidence in Data-MC agreement outside the signal region (in this case very low energy) and can be used as a constraint in a combined fit for sterile neutrino oscillations models.

In the following section a selection algorithm is described using a variation of the $1 e 1 \mathrm{P}$ algorithm. Externally triggered data are used to confirm the shape of the energy spectrum given the calibration. The Michel spectrum is then compared to the hit based algorithm developed for the MicroBooNE michel electron analysis [52]. This provides a unique benchmark to compare the energy scale and calibration between pixelized and hit based calorimetric schemes. Additionally, the expected $d E / d x$ and angular profile are studied. The energy smearing is estimated using a sample of MC michel electron decays. Finally, the 
spectrum is absolutely normalized and compared to the $5 \times 10^{19}$ POT data set.

\section{B.1.2 Selection}

Michel electrons are naturally vertexed in the reconstruction algorithm due to a vee shape being formed between track (muon) and shower (electron) collections of pixels. The SSNet algorithm has been shown to well separate electrons from muon produced in Michel decay in MicroBooNE LArTPC data images [8]. Michel electrons are reconstructed using the same cropped image scheme developed for the 1e1p analysis as described in Section 8.8. In each plane, cosmic rays are tagged by the line following algorithm. The nearest cosmic track which comes from the image boundary and stops closest to the vertex is tagged as the candidate muon track. The pixels for this track are placed back into the image. The same particle reconstruction algorithm is used to cluster particle pixels and match them across planes. The candidate particle clusters are then passed to an analysis and selection stage.

Michel electron candidate selection is straight forward inside the cropped image. The reconstruction algorithm has tagged a cosmic ray entering from the image boundary which has stopped at the center of the image where the vertexing algorithm has found a $3 \mathrm{D}$ vertex at the interface between track and shower pixels. To select candidate Michel electron events a set of square cuts is applied.

- Two sets of particles must be identified and matched across two or more planes. The candidate muon track is identified as the cluster which enters from the image boundary, and stops at the vertex location. The other particle is identified as the candidate Michel electron.

- The SSNet shower fraction for the electron cluster must be larger than 0.9. Due to the michel electron size, few topological features are available.

- In cases where the muon track and michel electron are fully contained inside the image, the muon track length must be larger than 30 centimeters. Additionally, the electron length must be larger than 15 pixels, or 4.5 centimeters.

The selection does not utilize any pattern recognition of the $d E / d x$ behavior near the muon bragg peak to determine if particle physically came to a stop before a secondary 
particle is omitted as in [52]. Based on handscan of unbiased detector data, excellent purity is observed to identifying Michel events. The reconstructed energy of the michel is estimated from calorimetry and shown in Fig. B.1 using a sample of unbiased cosmic ray data.

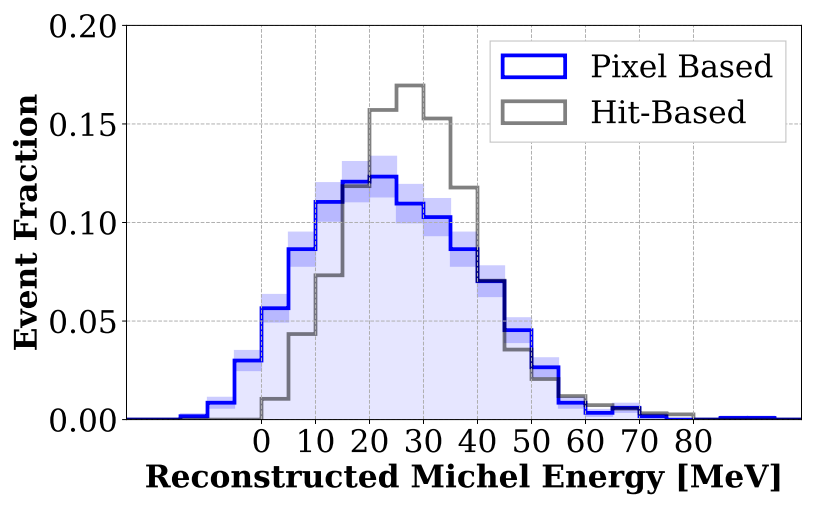

Figure B.1: Reconstructed Michel energy in MeV scale for the pixel based scheme (blue) applied to externally triggered data as described in Section B.1. The energy spectrum from the hit based identification scheme from is overlaid in grey.

The spectrum lies within the expected bounds of the expected Michel energy between 0 and approximately $60 \mathrm{MeV}$. From studying the lower energy bound on the Michel spectrum it can be seen that the nominal calibration offset allows the reconstructed electron energy to fall below $0 \mathrm{MeV}$ reconstructed energy by approximately 3 bins which represent a total fractional event rate of approximately 5 percent. The offset must decrease by approximately $10 \mathrm{MeV}$ to shift the spectrum to positive values. The two reconstruction schemes use different methods to calibrate the energy scale hence a difference in shape and peak position is expected. The peak position of the image based analysis (blue) differs of the publication (gray) by approximately $10 \mathrm{MeV}$. This shift can be accounted for by a shift of the spectrum left and right. If the image based spectrum is shifted to the right $10 \mathrm{MeV}$, the resulting spectrum will contain fractionally more events with higher Michel energy than the publication. This effect could be attributed to the hit threshold used in the publication. In the image based reconstruction all charge larger than 10 pixel value emitted of the particle trajectory are identified, potentially identifying charge clusters which are below the hit reconstruction threshold. In addition, the image based scheme likely collect more of the 
radiative photons in the energy regime above the critical energy with advanced clustering capability.

The $d E / d x$ spectrum is calculated by averaging the truncated $d Q / d x$ in the first 4 centimeters of the michel trajectory, applying the pixel to $\mathrm{MeV}$ calibration factor, and applying the average spatial correction. The result is shown in Fig. B.2 for data cosmic ray events.
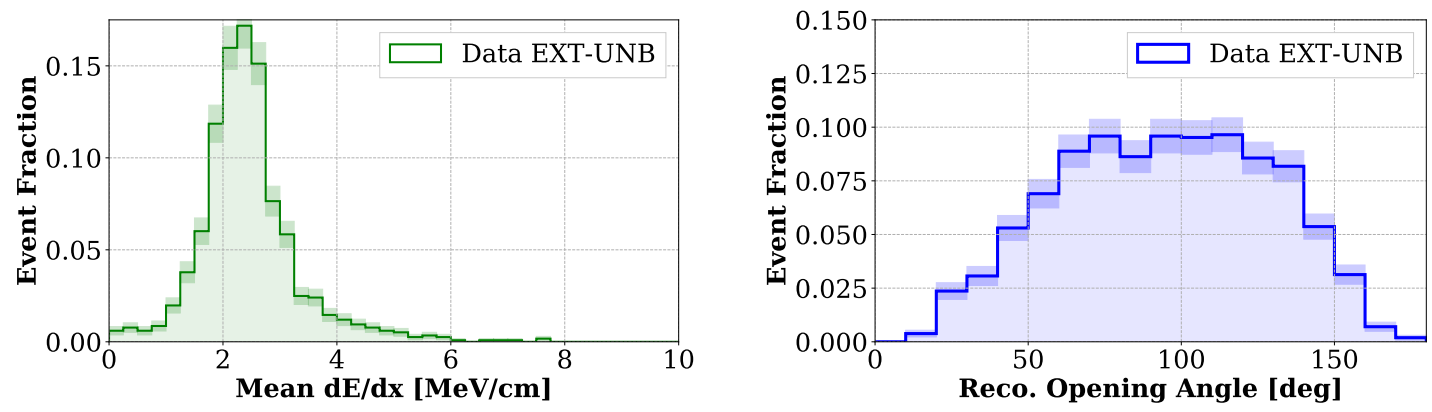

Figure B.2: Left: Reconstructed $d E / d x$ distribution for selecting Michel events in external data. The peak position lies in the expected MIP region. Right: Reconstructed opening angle between the muon and michel particle.

The peak position of the distribution lies just above the expected $2.2 \mathrm{MeV} / \mathrm{cm}$. The angular distribution between the muon and electron particle is also shown in Fig. B.2. In muon decay, michel electrons are emitted isotropically with a range of opening angle possible. The measured angular profile features a similar shape as the vertexing efficiency as a function of the neutrino daughter opening angle as shown in Fig. 8.51. The energy smearing is estimated by comparing the reconstructed to the true energy for a sample of simulated Michel events. The energy distributions are shown in Fig. B.3.

On average, the reconstructed energy is linearly correlated with the true energy with an approximately $10 \mathrm{MeV}$ bias observed.

\section{B.1.3 Data-MC Comparison}

The absolutely normalized event rate prediction for Michel production to the run $15 \times 10^{19}$ POT data sample. The result is shown in Fig. B.4. 

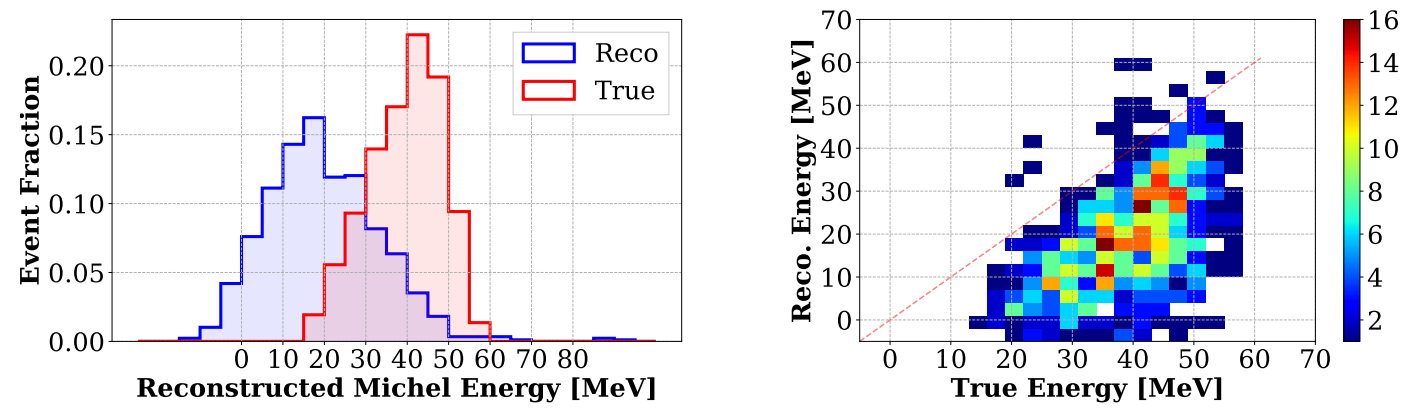

Figure B.3: Left: Reconstructed Michel spectrum (blue) for the selection applied to simulated michel decays. The red distribution shows the true michel energy deposited energy. Right: Correlation between the reconstructed and true michel energy. The red dashed line is shown at $y=x$ to guide the eye.

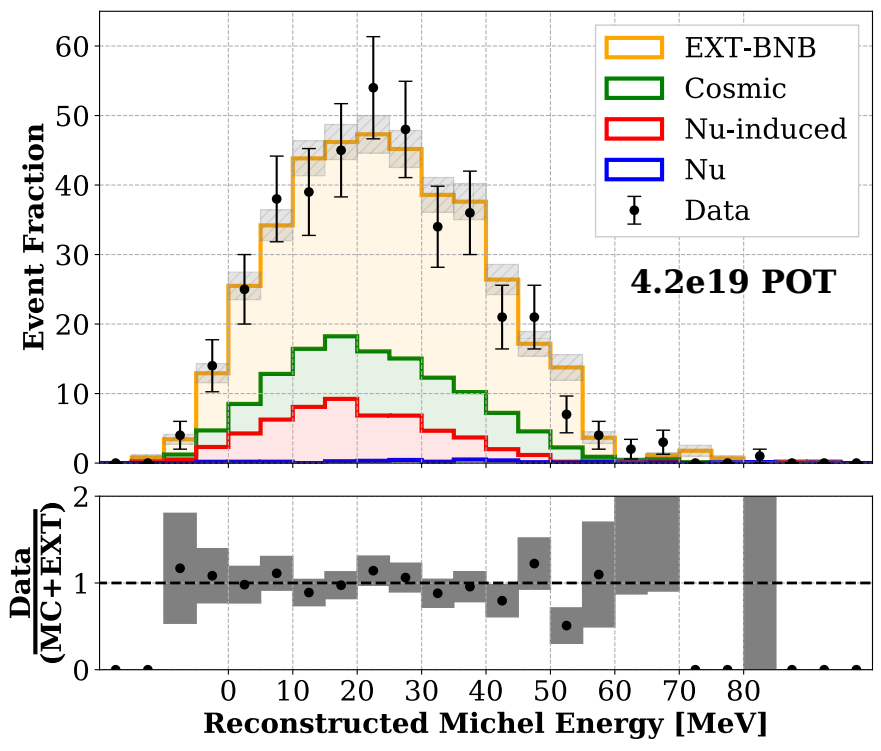

Figure B.4: Absolutely normalized Michel spectrum compared to the Run 1 data set. Good agreement is observed between data and prediction.

Four distributions are used to predict the event rate in the Run 1 dataset. The blue distribution represents the event rate for reconstructing neutrino interactions. The red distribution represents the expected event rate for selecting a Michel from a neutrino induced muon. The green distribution represents reconstructing a Michel electron on a cosmic 
ray muon. Finally, the orange distribution is the in-time reconstruction of cosmic ray Michel electrons (equivalent to the EXT-BNB sample). The black data points are the BNB triggered open dataset overlaid. The absolute normalization and shape agree between the data and prediction. 